\title{
27. ELEMENTAL COMPOSITION OF CENOZOIC PELAGIC CLAYS FROM DEEP SEA DRILLING PROJECT SITES 576 AND 578, WESTERN NORTH PACIFIC ${ }^{1}$
}

\author{
G. Ross Heath, Richard B. Kovar, Carlos Lopez, and Gregory L. Campi, Oregon State University²
}

\begin{abstract}
X-ray fluorescence analyses of 1143 samples from Site $576\left(32^{\circ} 21.4^{\prime} \mathrm{N}, 164^{\circ} 16.5^{\prime} \mathrm{E}\right)$ and 539 samples from Site 578 $\left(33^{\circ} 55.6^{\prime} \mathrm{N}, 151^{\circ} 37.7^{\prime} \mathrm{E}\right)$ for the elements $\mathrm{Na}, \mathrm{Mg}, \mathrm{Al}, \mathrm{Si}, \mathrm{P}, \mathrm{K}, \mathrm{Ca}, \mathrm{Ti}, \mathrm{Mn}, \mathrm{Fe}, \mathrm{Ba}$, and $\mathrm{S}$ show consistent trends from $\mathrm{Si}$-rich surficial deposits to dark brown clays rich in $\mathrm{Mn}, \mathrm{Fe}, \mathrm{P}$, and $\mathrm{Ti}$ in early Cenozoic sections. These data sets form the basis for a detailed paleogeochemical stratigraphy of North Pacific "red" clays.
\end{abstract}

\section{INTRODUCTION}

Deep Sea Drilling Project (DSDP) Sites 576 (between the Shatsky Rise and Emperor Seamounts at $32^{\circ} 21.4^{\prime} \mathrm{N}$, $164^{\circ} 16.5^{\prime} \mathrm{E}$ ) and 578 (between the Japan, French, and Shatsky Rise at $33^{\circ} 55.6^{\prime} \mathrm{N}, 151^{\circ} 37.7^{\prime} \mathrm{E}$; Fig. 1) were sampled continuously from the seafloor to chert (at 76 and $176 \mathrm{~m}$, respectively, below the seafloor) by means of the hydraulic piston core (HPC) during DSDP Leg 86.

At Site 576 , we recovered $27 \mathrm{~m}$ of buff clay over $29 \mathrm{~m}$ (27-56 m sub-bottom) of dark brown clay, which in turn overlay $20 \mathrm{~m}$ (56-76 m sub-bottom) of interbedded calcareous ooze and dark brown clay resting on chert of Campanian-Maestrichtian age.

At Site 578, we recovered a similar section of buff (77-125 m sub-bottom) and dark brown clay (125-176 m sub-bottom), overlain by $77 \mathrm{~m}$ of gray green, intermittently biosiliceous clay and ooze with numerous thin ash layers. At Site 578, the dark brown clay rests directly on Campanian chert at $176 \mathrm{~m}$ sub-bottom; the cored section contains no carbonate.

\section{METHODOLOGY}

Both sites were sampled at 10 - to 20 -cm intervals for paleomagnetic analysis (Heath, Rea, and Levi, this volume). After determination of remanent magnetization before and after alternating-field demagnetization, the $2 \times 2 \times 2 \mathrm{~cm}$ cubes of sediment were weighed, freezedried, weighed again (to yield water contents), and disaggregated by shaking with methacrylate spheres in polycarbonate sample tubes.

The resulting fine powder required no further grinding before being pressed at 10 tons pressure into $\mathrm{X}$-ray fluorescence (XRF) discs. The XRF analyses were carried out on a Phillips PW1600 simultaneous spectrometer. This instrument has 25 detectors, one per element, distributed around the sample and X-ray tube. Each detector-crystal system is optimized for the element it is

\footnotetext{
${ }^{1}$ Heath, G. R., Burckle, L. H., et al., Init. Repts. DSDP, 86: Washington (U.S. Govt. Printing Office).

2 Addresses: (Heath, present address) College of Ocean and Fishery Sciences, University of Washington, Seattle, WA 98195; (Kovar, Lopez, Campi) College of Oceanography, Oregon State University, Corvallis, OR 97331.
}

measuring, leading to high signal/noise ratios for all elements. The system is controlled by a DEC PDP 11/34 computer system which manages sample manipulation, data collection and archiving, data reduction through a fundamental parameters program (Chriss et al., 1978), and tabulation and plotting of elemental concentrations. The system has been calibrated by a set of 60 rock and sediment standards from the U.S. Geological Survey, National Bureau of Standards, Canada, and France, as well as by in-house pelagic clay standards that have been analyzed repeatedly by atomic absorption spectrophotometry and instrumental neutron activation analysis. These analyses indicate no systematic errors for the elements discussed.

\section{SUB-BOTTOM DEPTH OF SITE 576 SAMPLES}

Even at sea, visual examination and preliminary paleomagnetic analyses indicated that the nominal $100 \%$ recovery and sub-bottom depths reported for Holes 576 and $576 \mathrm{~B}$ by the drillers were not consistent with the sections recovered (see Site 576 chapter, this volume). Several problems were identified:

1. Incorrectly cored interval. Cores 2 and 3 of Hole 576A sampled the same interval. The reason is unknown, but may indicate a 1-stand drill pipe miscount on the drill floor.

2. Closure of the bumper subs as the sediments became stiffer with depth. This appears to have occurred in Core 6 of Hole 576, with a resultant reduction of the interval cored.

3. Uncertainties as to the location of "good" core within 9-m cored intervals.

4. Stretching ("flow-in") or compression of ductile clays within a core. Stretching is visually apparent due to elongation of sedimentary structures. Compression is difficult to demonstrate, but is the logical complement to stretching. These effects imply that the HPC piston was moving more slowly than the heave of the ship, suggesting malfunction of the HPC due either to premature failure of the shear pins or the stiffness of the clays.

5. Double cored sections (e.g., in Core 576B-3) also suggest heave velocities greater than piston velocities.

These problems led to actual recoveries of good ("undisturbed") sediment of about $80 \%$ in Holes 576 and 


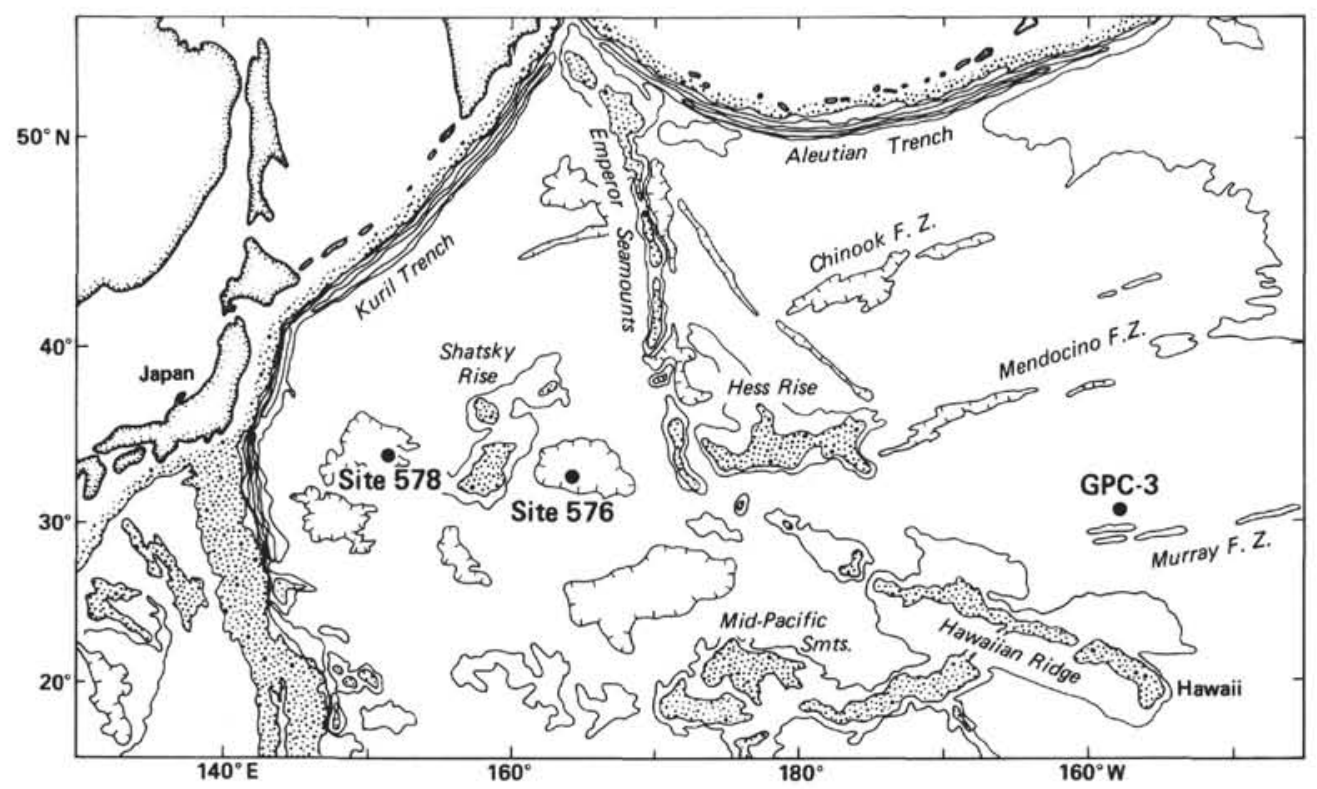

Figure 1. Location of DSDP Sites 576 and 578 relative to major physiographic features of the western North Pacific, and to LL44-GPC-3, a 24-m piston core studied in detail by Leinen (1979). Areas shallower than $4 \mathrm{~km}$ stippled; $5 \mathrm{~km}$ contour plain; $6 \mathrm{~km}$ contour hachured.

576B, rather than the nominal $100 \%$ recorded as the core was received on board. Because most of the Hole 576A sediment was taken in unopened sections of core liner for geotechnical measurements, the condition of this clay sequence is not well known. The presence of flow-in structures in several of the intervals remaining after geotechnical sampling suggests that the character of cores from Hole 576A is similar to that of the cores from the other two sites.

Based on detailed correlations of visual logs, paleomagnetic profiles, carbonate and intervening clay layers below $54 \mathrm{~m}$ sub-bottom, and profiles of elemental abundances, it has been possible to align the sediments recovered in the three holes, and create a composite section that represents virtually a complete sequence of the clay section at Site 576 .

Table 1 lists the sub-bottom depths of sections of good core from the three holes drilled. We emphasize that the Hole 576A corrections are much less certain than the Hole 576 and 576B corrections, because of the absence of paleomagnetic data and the unavailability of much of the cored material from Hole 576A. We also emphasize that the depths in Table 1 replace those presented in the Site 576 chapter, which were compiled before shorebased magnetic data or any geochemical profiles were available. Because Table 1 could not be prepared until late in the Leg 86 Initial Report production schedule, articles in the second part of this Initial Report volume that plot data versus adjusted depth have used adjusted depths from the Site 576 chapter that differ from those of Table 1 by as much as $4 \mathrm{~m}$.

The composite section contains only one notable gap, from 36.96 to $38.06 \mathrm{~m}$ sub-bottom. The discontinuities in a number of elemental profiles, particularly that of $\mathrm{Ti}$, across the gap suggest that it may be larger than the $1.1 \mathrm{~m}$ indicated. Because corrected depths for Hole
576B are deeper than driller's depths, whereas depths for Hole 576 below Core 2 are shallower, we have no real constraints on the magnitude of the missing section; $1.1 \mathrm{~m}$ is our best estimate, however.

\section{RESULTS}

Tables 2 to 6 list the analytical results from Holes $576,576 \mathrm{~A}$, and $576 \mathrm{~B}$, the 576 composite section based on the corrected depths of Table 1 and Hole 578, respectively. Except for $\mathrm{Na}$ and $\mathrm{S}$, which have uncertainties of 10 and $15 \%$, respectively, the precision of the tabulated values is within $2 \%$, and the accuracies within $5 \%$. For Hole $576 \mathrm{~A}$, the $\mathrm{Na}$ values are much noisier, resulting from detector problems, and are presented only to show general trends. Individual values should not be considered reliable.

Because of the length of time required to resolve the depth corrections for the Site 576 holes and the tight production schedule for the Leg 86 Initial Report volume, a full interpretation of the compositional data has been deferred to a future publication.

\section{ACKNOWLEDGMENTS}

We are grateful to Chris Dymond and Kerry O'Shea for preparing many of the XRF discs and for plotting and checking data. Donna Rea determined most of the water contents. Discussions with colleagues from the Leg 86 scientific party, particularly Joe Morley, Bob Jacobi, and Ulrich Bleil helped resolve some of the problems in assigning sub-bottom depths to cores from Holes 576 and 576B.

\section{REFERENCES}

Chriss, J. W., Birks, L. S., and Gilfrich, J. V., 1978. A versatile X-ray analysis program combining fundamental parameters and empirical coefficients. Anal. Chem., 50:33.

Leinen, M., 1979. Paleochemical signatures in Cenozoic Pacific sediments [Ph.D. dissert.]. Univ. Rhode Island, Kingston.

Date of Initial Receipt: 28 August 1984

Date of Acceptance: 25 September 1984 
Table 1. Key to convert section depths in Holes 576, 576A, and 576B to sub-bottom depths in centimeters.

\begin{tabular}{|c|c|c|c|}
\hline Section & $\begin{array}{l}\text { Depth } \\
\text { (cm) }\end{array}$ & $\begin{array}{l}\text { Adjusted } \\
\text { sub-bottom } \\
\text { depth } \\
(\mathrm{cm})\end{array}$ & Comments \\
\hline
\end{tabular}

Hole 576

$\begin{array}{llrl}1-1 & 0 & 64 & 0-64 \mathrm{~cm} \text { not recovered } \\ 1-2 & 0 & 214 & \\ 1, C \mathrm{CC} & 0 & 321 & 334-695 \mathrm{~cm} \text { not recovered }\end{array}$

$\begin{array}{lrrr}2-1 & 0 & 695 & \\ 2-2 & 0 & 845 & \\ 2-3 & 0 & 995 & \\ 2-4 & 0 & 1145 & \\ 2-5 & 0 & 1295 & \\ 2-5 & 110 & 1445 & 1405-1445 \mathrm{~cm} \text { missing } \\ 2-6 & 0 & 1485 & \\ 2-7 & 0 & 1635 & \\ 2, \mathrm{CC} & 0 & 1685 & \end{array}$

3-1 $\quad 0 \quad 1025$

3-2 $\quad 0 \quad 1175$

3-3 $\quad 0 \quad 1325$

3-4 $\quad 0 \quad 1475$

3-5 $\quad 0 \quad 1625$

$\begin{array}{lll}3, \mathrm{CC} & 0 & 1657\end{array}$

4-1 $\quad 70 \quad 1890$

4-2 $\quad 0 \quad 1970$

$4-3 \quad 0 \quad 2120$

4-4 $\quad 0 \quad 2270$

$4-5 \quad 0 \quad 2420$

$4-6 \quad 0=2570$

4-7 $\quad 0 \quad 2720$

$\begin{array}{lll}4, \mathrm{CC} & 0 & 2768\end{array}$

$2788-2800 \mathrm{~cm}$ not recovered

$\begin{array}{lrrr}5-1 & 40 & 2800 & \\ 5-5 & 48 & 3098 & 2910-3098 \mathrm{~cm} \text { not recovered } \\ 5-6 & 0 & 3200 & \\ 5-7 & 0 & 3350 & \\ 5, C C & 0 & 3399 & \end{array}$

6-3 $\quad 70 \quad 3800$

$\begin{array}{lll}6-4 & 0 & 3880 \\ 6-5 & 0 & 4030\end{array}$

$\begin{array}{lll}6-5 & 0 & 4030 \\ 6-6 & 0 & 4180\end{array}$

6-7 $\quad 0 \quad 4330$

$6, \mathrm{CC} \quad 0 \quad 4375$

$\begin{array}{lll}7-1 & 0 & 4340\end{array}$

$\begin{array}{lrr}7-2 & 50 & 4640\end{array}$

$\begin{array}{lll}7-3 & 0 & 4740\end{array}$

$\begin{array}{lll}7-4 & 0 & 4890\end{array}$

5040

5190

5340

5390

$\begin{array}{lrl}8-1 & 15 & 5425 \\ 8-2 & 0 & 5560 \\ 8-3 & 0 & 5710 \\ 8-4 & 0 & 5860 \\ 8-5 & 0 & 6010 \\ 8-6 & 0 & 6160 \\ 8-7 & 0 & 6310 \\ 8, C C & 0 & 6353 \\ & 4 & 6355\end{array}$

$5415-5425 \mathrm{~cm}$ not recovered

Hole $576,6379 \mathrm{~cm}$ total depth sampled
Table 1. (Continued).

\begin{tabular}{|c|c|c|c|}
\hline Section & $\begin{array}{l}\text { Depth } \\
\text { (cm) }\end{array}$ & $\begin{array}{l}\text { Adjusted } \\
\text { sub-bottom } \\
\text { depth } \\
\text { (cm) }\end{array}$ & Comments \\
\hline
\end{tabular}

Hole 576A

$\begin{array}{rrr}1-1 & 12 & 132 \\ 1-2 & 0 & 270 \\ 1-3 & 0 & 420 \\ 1-4 & 0 & 570 \\ 1-5 & 0 & 720 \\ 1-6 & 0 & 872 \\ 1, C C & 0 & 972\end{array}$

989-1075 cm not recovered

$\begin{array}{lrl}2-1 & 45 & 1075 \\ 2-2 & 0 & 1180 \\ 2-3 & 0 & 1330 \\ 2-4 & 0 & 1480 \\ 2-5 & 0 & 1630 \\ 2-6 & 0 & 1780 \\ 2-7 & 0 & 1930 \\ 2, C C & 0 & 1936\end{array}$

$1951-2300 \mathrm{~cm}$ not recovered

$\begin{array}{lrl}3-1 & 0 & 2300 \\ 3-2 & 0 & 2450 \\ 3-3 & 0 & 2600 \\ 3-4 & 0 & 2750 \\ & & \\ 3-6 & 100 & 2960 \\ 3-7 & 0 & 3010 \\ 3, \mathrm{CC} & 10 & 3040\end{array}$

$2700-\mathrm{cm}$ region disturbed $2755-2960 \mathrm{~cm}$ disturbed

2890-3065 cm overlap

$\begin{array}{lll}4-1 & 0 & 2890 \\ 4-2 & 0 & 3040\end{array}$

4-3 $\quad 0 \quad 3190$

4-4 $\quad 0 \quad 3340$

4-5 $\quad 0 \quad 3490$

4-6 $\quad 0 \quad 3640$

$\begin{array}{lll}4, \mathrm{CC} & 0 & 3768\end{array}$

$\begin{array}{lll}5-1 & 0 & 3910\end{array}$

4060

4210

4360

4510

4660

4810

$5, \mathrm{CC} \quad 15 \quad 4840$

$\begin{array}{lll}6-1 & 0 & 4825 \\ 6-2 & 0 & 4975\end{array}$

$4825-4863 \mathrm{~cm}$ overlap

5075-5270 cm (approx.) disturbed

$\begin{array}{lll}6-3 & 0 & 512 \\ 6-4 & 0 & 527\end{array}$

$6-5 \quad 0$

$\begin{array}{lll}6-7 & 0 & 5725 \\ 6, \mathrm{CC} & 0 & 5775\end{array}$

$5775-5798 \mathrm{~cm}$ disturbed

5795-5845 cm (approx.) disturbed 
G. R. HEATH, R. B. KOVAR, C. LOPEZ, G. L. CAMPI

Table 1. (Continued).

\begin{tabular}{|c|c|c|c|}
\hline Section & $\begin{array}{l}\text { Depth } \\
\text { (cm) }\end{array}$ & $\begin{array}{l}\text { Adjusted } \\
\text { sub-bottom } \\
\text { depth } \\
\text { (cm) }\end{array}$ & Comments \\
\hline
\end{tabular}

Hole 576A (Cont.)

Hole 576A, $6778 \mathrm{~cm}$ total depth sampled

Hole 576B

$\begin{array}{llr}1-1 & 0 & 0 \\ 1-2 & 0 & 150 \\ 1-3 & 0 & 300 \\ 1-4 & 0 & 450 \\ 1-5 & 0 & 600 \\ 1-6 & 0 & 750 \\ 1, C C & 0 & 807\end{array}$

$821-1205 \mathrm{~cm}$ not recovered

$\begin{array}{lll}2-1 & 5 & 1205 \\ 2-2 & 0 & 1350 \\ 2-3 & 0 & 1500\end{array}$

$1600-1685 \mathrm{~cm}$ disturbed

$\begin{array}{lrr}2-4 & 35 & 1685 \\ 2-5 & 0 & 1800 \\ 2-6 & 0 & 1950 \\ 2-7 & 0 & 2100 \\ 2 . C C & 0 & 2148\end{array}$

3-1 $\quad 0 \quad 2150$

3-1 $\quad 71 \quad 204$

3-2 $\quad 0 \quad 2110$

$3-2 \quad 71 \quad 227$

$3-3 \quad 0 \quad 2350$

3-6 $\quad 21 \quad 2787$

$\begin{array}{lrl}3-7 & 0 & 2916\end{array}$

$2988-3150 \mathrm{~cm}$ not recovered

$\begin{array}{lrl}4-3 & 100 & 3150 \\ 4-4 & 0 & 3200 \\ 4-5 & 0 & 3350 \\ 4-6 & 0 & 3500 \\ 4-7 & 0 & 3650 \\ 4, C C & 0 & 3702\end{array}$

2041-2160 cm overlap

2041-2181 cm repeated

2221-2271 cm not recovered
Table 1. (Continued).

\begin{tabular}{|c|c|c|c|}
\hline Section & $\begin{array}{l}\text { Depth } \\
\text { (cm) }\end{array}$ & $\begin{array}{l}\text { Adjusted } \\
\text { sub-bottom } \\
\text { depth } \\
\text { (cm) }\end{array}$ & Comments \\
\hline
\end{tabular}

Hole 576B (Cont.)

$3724-3930 \mathrm{~cm}$ not recovered

$\begin{array}{lrrr}5-2 & 0 & 3930 & \\ 5-3 & 0 & 4080 & \\ 5-3 & 20 & 4140 & 4100-4140 \mathrm{~cm} \text { not recovered } \\ 5-3 & 75 & 4255 & 4195-4255 \mathrm{~cm} \text { not recovered } \\ 5-4 & 0 & 4330 & \\ 5-5 & 0 & 4480 & \\ 5-6 & 0 & 4630 & \\ 5, C C & 2 & 4755 & 4775-4940 \mathrm{~cm} \text { not recovered }\end{array}$

$\begin{array}{lrrl}6-1 & 110 & 4940 & \\ 6-2 & 0 & 4980 & \\ 6-3 & 0 & 5130 & 5220-5300 \mathrm{~cm} \text { missing } \\ 6-3 & 90 & 5300 & \\ 6-4 & 0 & 5360 & 5430-5516 \mathrm{~cm} \text { missing } \\ 6-4 & 70 & 5516 & \\ 6-5 & 0 & 5596 & \\ 6-6 & 0 & 5746 & \\ 6, C C & 10 & 5888 & 5901-5913 \mathrm{~cm} \text { not recovered }\end{array}$

$\begin{array}{rrr}7-1 & 5 & 5913 \\ 7-2 & 0 & 6058 \\ 7-3 & 108 & 6208 \\ 7-4 & 0 & 6250 \\ 7-5 & 0 & 6400 \\ 7-6 & 0 & 6550 \\ 7-7 & 0 & 6700 \\ 7, \mathrm{CC} & 0 & 6734\end{array}$

$6771-6860 \mathrm{~cm}$ not recovered

$\begin{array}{rrr}8-1 & 115 & 686 \\ 8-2 & 0 & 689 \\ 8-3 & 0 & 704 \\ 8-4 & 0 & 719 \\ 8-5 & 0 & 734 \\ 8-6 & 0 & 749\end{array}$

$7500-7610 \mathrm{~cm}$ not recovered

$9, \mathrm{CC}$

7610
Hole 576B, $7610 \mathrm{~cm}$ total depth sampled 
Table 2. Elemental abundance (wt. \%) and water content (\% dry wt.) of Hole 576 sediment samples.

\begin{tabular}{|c|c|c|c|c|c|c|c|c|c|c|c|c|c|c|c|c|c|}
\hline Lab ID & $\begin{array}{l}\stackrel{H}{~} \\
\stackrel{0}{0}\end{array}$ & 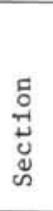 & 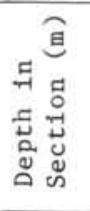 & 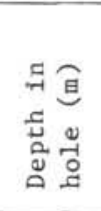 & $\mathrm{Na}$ & $\mathrm{Mg}$ & A1 & Si & P & $\mathrm{K}$ & $\mathrm{Ca}$ & $\mathrm{Ti}$ & Mn & Fe & $\mathrm{Ba}$ & $\mathrm{S}$ & $\mathrm{H}_{2} \mathrm{O}$ \\
\hline DP1 4294 & 1 & 1 & 0.11 & 0.75 & 0.62 & 1.86 & 7.43 & 6.3 & 0.062 & 2.50 & 0.46 & 0.412 & 0.47 & 4.77 & 0.091 & .020 & 141 \\
\hline DP1 4295 & 1 & 1 & 0.21 & 0.85 & 0.58 & 1.88 & 7.31 & & 0.067 & .52 & 0.53 & .409 & 0.45 & 4.74 & & & 130 \\
\hline 296 & 1 & 1 & 0.31 & 0.95 & 0.52 & 1.90 & 7.50 & & 0.058 & 2.59 & 0.45 & 0.416 & 0.21 & 4.86 & 0.086 & 0.035 & 142 \\
\hline DP1 4297 & 1 & 1 & 0.41 & 05 & 0.59 & 1.85 & 7.48 & 26.4 & 0.059 & 2.56 & 0.53 & 0.415 & 0.31 & 4.71 & .087 & & 139 \\
\hline DP1 4298 & 1 & 1 & 0.51 & 1.15 & 0.60 & 1.89 & 7.59 & 26.4 & 0.060 & 2.57 & 0.51 & 0.418 & 0.33 & 4.82 & 0.090 & 0.026 & 146 \\
\hline DP1 4299 & 1 & 1 & 0.61 & 1.25 & 0.56 & 1.91 & 7.58 & 26.1 & 0.060 & 2.59 & 0.49 & 0.419 & 0.35 & 4.86 & 0.093 & 0.030 & 132 \\
\hline DP1 4300 & 1 & 1 & 0.71 & 1.35 & 0.56 & 1.89 & 7.44 & 25.9 & 0.059 & 2.57 & 0.52 & 0.423 & 0.37 & 4.81 & 0.085 & 0.040 & 141 \\
\hline DP1 4301 & 1 & 1 & 0.81 & 1.45 & 0.49 & 1.89 & 7.50 & 26.2 & 0.059 & 2.57 & 0.54 & 0.422 & 0.42 & 4.69 & 0.086 & 0.053 & 152 \\
\hline DP1 4302 & 1 & 1 & 0.91 & 1.55 & 0.53 & 1.93 & 7.68 & 26.2 & 0.062 & 2.57 & 0.53 & 0.427 & 0.56 & 4.83 & & & 139 \\
\hline 303 & 1 & 1 & 1.01 & 65 & 0.56 & 1.95 & 7.60 & 26 & 0.061 & 2.57 & 9 & & & 4.75 & 0 & & 139 \\
\hline DP1 4304 & 1 & 1 & 1.11 & 1.75 & 0.52 & 1.91 & 7.43 & 26.6 & 0.056 & 2.48 & 0.43 & 0.409 & 0.74 & 4.66 & 97 & 0.026 & 174 \\
\hline P14305 & 1 & 1 & 1.21 & 1.85 & 0.40 & 1.91 & 7.37 & 26.8 & 0.053 & 2.52 & 0.45 & 0.414 & 0.38 & 4.65 & & & 177 \\
\hline 14306 & 1 & 1 & 1.31 & 1.95 & 0.44 & 1.89 & 7.36 & 26.8 & 0.053 & 2.50 & 0.39 & 0.410 & 0.34 & 4.66 & 0.091 & 0.023 & 192 \\
\hline P14307 & 1 & 1 & 1.41 & 2.05 & 0.49 & 1.87 & 7.34 & & 0.054 & 2. & & 0. & & 4.63 & & & 170 \\
\hline DP1 4309 & 1 & 2 & 0.16 & 2.30 & 0.42 & 1.89 & 7.31 & 26.9 & 0.051 & 2.58 & 0. & 0.4 & 0.14 & 4.65 & .087 & 51 & 174 \\
\hline DP1 4310 & 1 & 2 & 0.22 & 2.36 & 0.44 & 1.88 & 7.42 & 27 & 0.0 & 2.56 & & 0.4 & 0. & 4.65 & 6 & 1 & 177 \\
\hline 311 & 1 & 2 & 0.31 & 2 & 0.41 & 1. & 7.2 & 26 & 0.0 & 2.57 & & 0. & 2 & 4. & 39 & 63 & 189 \\
\hline DP1 4312 & 1 & 2 & 0.41 & 2.55 & 0.41 & 1.87 & 7. & 27.0 & 0. & 2. & 0. & 0.400 & 0.36 & 4.53 & 2 & 0.031 & 220 \\
\hline DP14313 & 1 & 2 & 0.51 & 2.65 & 0.43 & 1.91 & 7.32 & 27.0 & 0.051 & 2.59 & 0.43 & 0.408 & 0.27 & 4.61 & 37 & 0. & 185 \\
\hline DP14314 & 1 & 2 & 0.61 & 2.75 & 0.44 & 1.89 & 7.39 & 26.9 & 0.052 & 2.60 & 0.43 & 0.4 & 0.43 & 4.61 & & & 179 \\
\hline DP1 4315 & 1 & 2 & 0.71 & 2.85 & 0.51 & 1.87 & 7.30 & 26.9 & 51 & & 0. & 0.4 & 0.25 & 4.62 & 33 & 59 & 162 \\
\hline DP1 4316 & 1 & 2 & 0.81 & 2.95 & 0.64 & 2.06 & 8.20 & 29 & 0.0 & 2. & 0. & 0.4 & 0.22 & 5.35 & & 5 & 164 \\
\hline DP14317 & 1 & 2 & 0.91 & 3.05 & 0.49 & 1.88 & 7.4 & 26 & 0.053 & 2. & 0. & $0.1+x$ & 0. & 4 & 39 & & 165 \\
\hline 319 & 1 & 2 & 1.01 & 3 & 0.52 & 1 & 7.5 & 26 & 0.053 & 2. & 6 & 0.424 & 0.39 & 4.74 & 39 & 33 & 162 \\
\hline DP1 4318 & 1 & C & 0.07 & 3.2 & 0.63 & 2. & 8. & 30 & 2 & 2. & 0.57 & 0.470 & 0.58 & 5.48 & 0. & $\$ 1$ & 170 \\
\hline DP1 4320 & 2 & 1 & 0.06 & 7.01 & 0.53 & 2.01 & 7.61 & 26.1 & 0.051 & 2.69 & 0.45 & 0.425 & 0.63 & 4.79 & 1 & 50 & 134 \\
\hline DP1 4321 & 2 & 1 & 0.16 & 7.11 & 0.55 & 2.03 & 7.60 & 26.4 & 0.047 & 2.73 & 0.49 & 0.430 & 0.28 & 4.80 & 35 & 0. & 149 \\
\hline 322 & 2 & 1 & 0.26 & 7.21 & 0.52 & 1.97 & 7.59 & 26.4 & 0.050 & 2.66 & 0.44 & 0.417 & 0.36 & 4.70 & 38 & 50 & 159 \\
\hline DP1 4323 & 2 & 1 & 0.36 & 7.31 & 0.59 & 1.98 & 7.48 & 26.2 & 0.050 & & 0. & 0. & 0.44 & 4.68 & 0. & 88 & 145 \\
\hline DP1 4324 & 2 & 1 & 0.46 & 7.41 & 0.51 & 2.01 & 7.56 & 26.4 & 0.053 & 2. & 0. & 0.4 & 0.38 & 4.70 & 1 & 1 & 158 \\
\hline DP1 4325 & 2 & 1 & 0.56 & 7.51 & 0.57 & 2.04 & 7.59 & 26 & 0.052 & 2. & 0. & 0.1 & 0.33 & 4.80 & 32 & 8 & 144 \\
\hline DP14326 & 2 & 1 & 0.66 & 7.61 & 0.48 & 2.03 & 7.63 & 26 & 1 & 2. & 3 & 0. & 0.28 & & 6 & & 43 \\
\hline DP & 2 & 1 & 0.76 & 7. & 0.56 & 2.08 & 7. & 3 & 0.053 & 2. & 8 & 31 & 7 & 3 & 1 & 5 & 146 \\
\hline 328 & 2 & 1 & 0.86 & 7. & 0.52 & 2 & 7. & 26 & 0 & 2 & & 0. & 5 & 4.86 & 36 & 2 & 144 \\
\hline P1 4329 & 2 & 1 & 0.96 & 7. & 0.46 & 2.01 & 7.53 & 26.1 & 51 & 2. & 0. & 0.418 & 0.29 & 4.76 & 0.090 & 58 & 159 \\
\hline DP1 4330 & 2 & 1 & 1.06 & 8.0 & 0.44 & 2.04 & 7.58 & 26.1 & 0.050 & 2.70 & 0.42 & 0.414 & 0.31 & 4.75 & 0.095 & 0. & 159 \\
\hline DP1 4331 & 2 & 1 & 1.16 & 8.11 & 0.59 & 2.07 & 7.64 & $26 \cdot 3$ & 0.051 & 2.78 & 0.50 & 0.430 & 0.32 & 4.87 & 0.095 & 0. & 146 \\
\hline DP14332 & 2 & 1 & 1.26 & 8.21 & 0.43 & 2.05 & 7.62 & 26.2 & 0.053 & 2.67 & 0.44 & 0.417 & 0.38 & 4.77 & 0. & & 168 \\
\hline DP14333 & 2 & 1 & 1.36 & 8.31 & 0.39 & 2.01 & 7.49 & 26.4 & 0.052 & 2.61 & 0.46 & 0.408 & 0.39 & 4.65 & 0. & 73 & 178 \\
\hline DP1 4334 & 2 & 1 & 1.46 & 8.41 & 0.48 & 1.98 & 7.58 & 26.2 & 0.057 & 2.59 & 0.42 & 0.413 & 0.39 & 4.71 & 8 & 6 & 154 \\
\hline 335 & 2 & 2 & 0.06 & 8 & 9 & 2 & 7. & 2 & 5 & 2.65 & 5 & 8 & 4 & 7 & 9 & 9 & 145 \\
\hline 336 & 2 & 2 & 0.16 & 8.6 & 6 & 1 & 7. & .2 & 54 & 2.61 & 0.47 & 0.418 & 0.39 & 4.79 & 0.0 & 49 & 147 \\
\hline DP1 4337 & 2 & 2 & 0.26 & 8.71 & 0.45 & 1.96 & 7.59 & 26.4 & 0.053 & 2.58 & 0.46 & 0.414 & 0.39 & 4.77 & 0.087 & 0. & 159 \\
\hline DP1 4338 & 2 & 2 & 0.36 & $8.8-2$. & 0.47 & 2.01 & 7.58 & 26.2 & 0.055 & 2.61 & 0.43 & 0.416 & 0.41 & 4.75 & 0.094 & 0.048 & 156 \\
\hline DP1 4339 & 2 & 2 & 0.46 & 8.91 & 0.46 & 1.99 & 7.60 & 26.5 & 0.054 & 2.60 & 0.41 & 0.413 & 0.42 & 4.74 & 0.096 & 0.029 & 161 \\
\hline DP1 4340 & 2 & 2 & 0.56 & 9.01 & 0.46 & 2.00 & 7.5 & 26.3 & 0.053 & 2.61 & 0.40 & 0.414 & 0.29 & 4.74 & 0.094 & 0.041 & 165 \\
\hline DP1 4341 & 2 & 2 & 0.66 & 9.11 & 0.46 & 2.06 & 7.54 & 26.3 & 0.048 & 2.68 & 0.47 & 0.418 & 0.39 & 4.78 & 0.090 & 0. & 161 \\
\hline DP1 4342 & 2 & 2 & 0.76 & 9.21 & 0.45 & 1.98 & 7.48 & 26.3 & 0.049 & 2.59 & 0.42 & 0.410 & 0.39 & 4.72 & & & 170 \\
\hline DP1 4343 & 2 & 2 & 0.86 & 1 & 0.32 & 2.03 & 7.36 & 26.7 & 0.050 & 2.58 & 0.42 & 0.398 & 0.26 & 4. & & & 207 \\
\hline+344 & 2 & 2 & 0.96 & & .37 & & & 26.5 & & & 0. & 0.2 & 6 & 4. & & & 194 \\
\hline DP14345 & 2 & 2 & 1.06 & 9.51 & 0.49 & 2.02 & 7.51 & 26.4 & 0.058 & 2.64 & 0.55 & 0.422 & 0.46 & 4.72 & 0.085 & 0.082 & 163 \\
\hline DP14346 & 2 & 2 & 1.16 & 9.61 & 0.47 & 1.96 & 7.46 & $26 . ?$ & 0.056 & 2.55 & 0.44 & 0.406 & 0.40 & 4.65 & 0.085 & 0.041 & 170 \\
\hline DP1 4347 & 2 & 2 & 1.26 & 9.71 & 0.53 & 2.03 & 7.46 & 26.2 & 0.053 & 2.67 & 0.54 & 0.418 & 0.36 & 4.75 & 0.093 & & 149 \\
\hline DP1 4348 & 2 & 2 & 1.36 & 9.81 & 0.48 & 1.97 & 7.46 & 26.2 & 0.054 & 2.61 & 0.46 & 0.412 & 0.37 & 4.71 & & & 154 \\
\hline DP1 4349 & 2 & 2 & 1.46 & 9.91 & 0.69 & 2.04 & 7.53 & 26.1 & 0.050 & 2.76 & 0.51 & 0.429 & 0.39 & 4.83 & 0. & & 154 \\
\hline DP1 4350 & 2 & 3 & 0.06 & 0 & .50 & & & 26.0 & & & 0.41 & 0. & & 4.75 & 9 & & 176 \\
\hline DP1 & 2 & 3 & 0.16 & & .50 & 2 & 7.4 & 26.1 & 0.050 & 2.72 & 0.47 & 0.414 & 0.38 & 4.70 & 0.101 & 0.118 & 185 \\
\hline DP1 4352 & 2 & 3 & 0.26 & 10.21 & 0.40 & 1.98 & 7.4 & 26.4 & 0.051 & 2.55 & 0.36 & 0.396 & 0.30 & 4.55 & 0.095 & 0.051 & 218 \\
\hline DP14353 & 2 & 3 & 0.36 & 10.31 & 0.29 & 1.83 & 6.98 & $27 \cdot 3$ & 0.052 & 2.43 & 0.39 & 0.389 & 0.52 & 4.40 & 0.095 & 0.056 & 218 \\
\hline DP1 4354 & 2 & 3 & 0.46 & 10.41 & 0.55 & 1.90 & 7.44 & 26.4 & 0.057 & 2.52 & 0.43 & 0.409 & 0.49 & 4.64 & 0.086 & 0.033 & 180 \\
\hline DP1 4355 & 2 & 3 & 0.56 & 10.51 & 0.51 & 1.95 & $7 \cdot 37$ & 26.3 & 0.053 & 2.59 & 0.53 & 0.410 & 0.44 & 4.62 & 0.089 & 0.074 & 163 \\
\hline DP14356 & 2 & 3 & 0.66 & 10.61 & 0.55 & 1.94 & 7.41 & 26.2 & 0.051 & 2.60 & 0.50 & 0.408 & 0.41 & 4.67 & 0.087 & & 142 \\
\hline DP1 4357 & 2 & 3 & 0.76 & 10.71 & 0.60 & 1.91 & 7.31 & 26.5 & 0.048 & 2.63 & 0.57 & 0.402 & 0.37 & 4.48 & 0.087 & 0.073 & 138 \\
\hline
\end{tabular}


Table 2. (Continued).

\begin{tabular}{|c|c|c|c|c|c|c|c|c|c|c|c|c|c|c|c|c|c|}
\hline Lab ID & 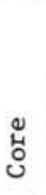 & 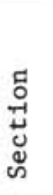 & 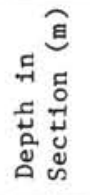 & 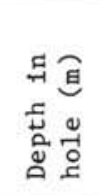 & $\mathrm{Na}$ & $\mathrm{Mg}$ & A1 & Si & $\mathrm{P}$ & K & $\mathrm{Ca}$ & $\mathrm{Ti}$ & Mn & $\mathrm{Fe}$ & $\mathrm{Ba}$ & $\mathrm{S}$ & $\mathrm{H}_{2} \mathrm{O}$ \\
\hline 358 & 2 & 3 & 0.86 & .81 & 0.59 & 1.78 & 7.15 & 26.6 & 0.046 & 2.55 & 0.57 & 73 & 0.35 & 4.13 & 0.089 & 0.042 & 122 \\
\hline 359 & 2 & 3 & 96 & .91 & & & & & & & & & & & 0.083 & & 127 \\
\hline 14360 & 2 & 3 & 1.06 & 1.01 & 0. & & 7 & & & & & & & 4.70 & 0.092 & & 146 \\
\hline+361 & 2 & 3 & 1.16 & 11.11 & 0.57 & 2.07 & 1.30 & 20.1 & 0.050 & 2.11 & 0.40 & 31 & 0.46 & 4.81 & 0.089 & .085 & 149 \\
\hline 14362 & 2 & 3 & 1.26 & 11.21 & 0.56 & 2.03 & 7.61 & 26.3 & 0.045 & 2.70 & 0.40 & 0.421 & 0.38 & 4.73 & 0.090 & & 157 \\
\hline DP1 4363 & 2 & 3 & 1.36 & 11.31 & 0.46 & 2.05 & 7.52 & 26.3 & 0.048 & 2.67 & 0.44 & 0.421 & 0.28 & 4.74 & 0.095 & 0.081 & 168 \\
\hline DP1 4364 & 2 & 3 & 1.46 & 11.41 & 0.55 & 2.00 & 7.58 & & 0.047 & 2.70 & 0.38 & & & 4.81 & 0.092 & & 147 \\
\hline DP14365 & 2 & 4 & 0.06 & 11.51 & 0.51 & 2.04 & 7. & & 0.053 & & & & & 4.76 & 4 & 0.072 & 155 \\
\hline 66 & 2 & 4 & 0.16 & 11.61 & 0.45 & 2.01 & 7.58 & & 0.056 & 2.61 & & 5 & & 4.69 & 0. & & 165 \\
\hline 367 & 2 & 4 & 0.26 & 71 & 0 & 1. & 7. & & 0 & & & & & 4. & 0 & & 181 \\
\hline 368 & 2 & 4 & 0.36 & 11.81 & & & 7. & & & & & & & & & & 164 \\
\hline & 2 & 4 & 0.46 & 11.91 & 0.49 & 9 & & & & 2 & 0. & & & 1 & 0.092 & 0 & 149 \\
\hline & 2 & 4 & 0.56 & 12.01 & 0.54 & 1.98 & 1.43 & 26.4 & 0.054 & 2.62 & 0.43 & 0.405 & 1 & 4.55 & 0.095 & & 140 \\
\hline 371 & 2 & 4 & 0.66 & 12.11 & 0.65 & 1.48 & 6.64 & 27.5 & 0.045 & 3.02 & 0.44 & 0.275 & 0. & 3.25 & 0.090 & & 119 \\
\hline DP1 4372 & 2 & 4 & 0.76 & 12.21 & 0.49 & 2.00 & 7. & & $0.8-1$ & & & & & & & & 178 \\
\hline 14373 & 2 & 4 & 0.86 & 12.31 & 0.37 & 2.0 & 7. & & 0.0 & & & & & & & & 177 \\
\hline 74 & 2 & 4 & 0.96 & 12.41 & 0.1 & 2. & 7. & & 0 & 5 & & & & & & & 165 \\
\hline 375 & 2 & 4 & 1.0 & 12.51 & 0. & $?$ & 7. & & 3 & 9 & & & & & & & 174 \\
\hline 76 & 2 & 4 & 1.16 & 12.61 & & & & & & & & & b & 4.59 & 2 & 1 & 180 \\
\hline & 2 & 4 & 1.26 & 12.71 & 0.42 & 2.01 & 7. & 26.4 & 0.055 & 2.56 & 0.43 & 5 & 0. & 4.6 & 0. & 3 & 175 \\
\hline 378 & 2 & 4 & 1.36 & 12.81 & 0.37 & 2.03 & 7.53 & 26.2 & .051 & 2.59 & 0.35 & 1 & 0. & 4.70 & 0 & & 76 \\
\hline DP1 4379 & 2 & 4 & 1.46 & 12.91 & 0.33 & .11 & 7.5 & & .054 & 2.62 & 0.39 & & 8 & 4.67 & & & 185 \\
\hline DP1 4380 & 2 & 5 & 0.06 & 13.01 & 0.3 & 0 & & & p & 2.54 & & & & 4.64 & & & 202 \\
\hline 81 & 2 & 5 & 0.16 & 13.11 & 0 & 2.06 & 7. & 2 & 6 & 2. & & & & & & & 98 \\
\hline 32 & 2 & 5 & 0.2 & 13. & & & & & & & & & & & & & 76 \\
\hline 83 & 2 & 5 & 0.36 & 13 & & & & & & & & & 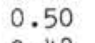 & 4. & & & 183 \\
\hline 84 & 2 & 5 & 0.46 & 13.41 & & & & & & & & 0 & 8 & 4.59 & 0. & 3 & 192 \\
\hline 85 & 2 & 5 & 0.56 & 13.51 & 0.35 & .06 & 7.50 & 26.4 & .053 & 2.62 & 0.36 & 0.400 & .42 & 4.58 & 0 . & & 181 \\
\hline 386 & 2 & 5 & 0.66 & 13.61 & 0.40 & .02 & 7.53 & & .052 & 2.55 & & 6 & .41 & 4.57 & 0. & & 195 \\
\hline DP1 4387 & 2 & 5 & 0.76 & 13.71 & 0.28 & 2 . & & & 3 & 2.48 & 0 & & 3 & 4.52 & $3 r$ & & 220 \\
\hline 388 & 2 & 5 & 0.86 & 13.81 & 0.4 & 19 & 7. & & 3 & 2.4 & 1 & & 1 & 9 & 0. & 8 & 192 \\
\hline & 2 & 5 & 0.96 & 13.91 & 0.4 & 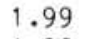 & & & 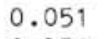 & 2 & & & 8 & 7 & & & 71 \\
\hline & 2 & 5 & 1.0 & 14. & & 9 & & & & & & & & 3 & & & 88 \\
\hline 1 & 2 & 5 & 1.1 & 1 & & & & & & & & 2 & 0. & 4. & 0 & & 54 \\
\hline 392 & 2 & 5 & 1.26 & 14.61 & 0.4 & 9 & 7. & 26.4 & 0.046 & 2.61 & 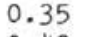 & 5 & 0.4 & 4.71 & 0. & & 172 \\
\hline 393 & 2 & 5 & 1.36 & 14.71 & 0.42 & .09 & 7.60 & 26.6 & .050 & 2.64 & 0.42 & 0.416 & 0.43 & 4.63 & 9 & & 172 \\
\hline 394 & 2 & 5 & 1.46 & 14.81 & 0.47 & .04 & 7.64 & 26.3 & .052 & 2.58 & 0.3 & 0.409 & 0.45 & 4.65 & & 0. & 178 \\
\hline 4395 & 2 & 6 & 0.06 & 14.91 & 0.41 & .07 & 7.63 & 26.4 & .053 & 2.63 & 0.38 & 0. & 0. & 4.64 & & 0. & 172 \\
\hline 4396 & 2 & 6 & 0.16 & 15.01 & 0.36 & 03 & 7.58 & 26 & 054 & 2.62 & 0 . & 0.3 & & 4.5 & & & 175 \\
\hline & 2 & 6 & 0.26 & 15.11 & & & 7.5 & & 5 & 2.5 & & & & & & & 178 \\
\hline & 2 & 6 & 0.36 & & & & & & & & & & & & & & 197 \\
\hline 9 & 2 & 6 & 46 & 1 & & & & & & 2. & & & 6 & 5 & 5 & 0. & 172 \\
\hline 00 & 2 & 6 & 0.56 & 15.41 & 0.43 & 2.06 & 7. & 26.3 & .057 & 2 & 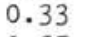 & 4 & 2 & 4. & 0.098 & 0.0 & 182 \\
\hline 01 & 2 & 6 & 0.66 & 15.51 & .35 & 4 & 7.5 & 26. & 7 & 2.45 & 0 & 7 & 0.53 & 4.55 & 7 & & 196 \\
\hline 4402 & 2 & 6 & 0.76 & 15.61 & .34 & .07 & 7.5 & 26.3 & .055 & 2.46 & 0 & 0.398 & 0.50 & 4.53 & 0.095 & 0.021 & 194 \\
\hline DP1 4403 & 2 & 6 & 0.86 & 15.71 & 0.25 & 2.07 & 7.5 & 26. & 6 & 2.5 & 0.37 & 07 & 0.48 & 4.56 & 0.094 & 0.0 & 200 \\
\hline & 2 & 6 & 0.96 & 15.81 & 0.4 & 2.04 & 7.5 & & 0 & 2.4 & & & & & & & 04 \\
\hline & 2 & 6 & & & & 9 & 7. & & 8 & 2.45 & & & & 4. & & & 202 \\
\hline & 2 & 6 & & & & & & & & & & & & & & 2 & 191 \\
\hline & 2 & 6 & 6 & & & & & & & & & 3 & 0 & 4. & & & 190 \\
\hline 8 & 2 & 6 & 1.36 & 1 & & & & & & & & 6 & 0.46 & 4 & & 2 & 176 \\
\hline DP14409 & 2 & 6 & 1.46 & 6.31 & 0.38 & 3 & 7.6 & 0 & & 2.56 & 0 & 0.418 & 0.40 & 4. & 0. & 7 & 165 \\
\hline 10 & 2 & 7 & 0.06 & 16.41 & 0.45 & 0 & 7. & 26 & 0.05 & 2.56 & 0. & 0.418 & 0.43 & 4.72 & & 0.039 & 165 \\
\hline 4411 & 2 & 7 & 0.16 & .51 & 0 & .09 & 7. & 5. & 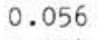 & 2.62 & 0.41 & 0.419 & 0.43 & 4.72 & 0.094 & 0.037 & 163 \\
\hline DP14412 & 2 & 7 & 0.26 & 16.61 & 0.41 & & & 2 & 4 & 2.49 & 0.38 & 0.406 & 0.49 & 4.67 & 0.091 & & 186 \\
\hline DP1 4413 & 2 & 7 & 0.36 & & 0 & & & 26.3 & & 2.49 & & & & & & & \\
\hline 414 & 2 & 7 & 0.46 & 16.81 & & & & & & & & 0.410 & 0.51 & 4.70 & & 0. & \\
\hline 415 & 3 & 1 & 0.26 & 10.51 & & & 7.5 & & 0.057 & 2.61 & & & 0.41 & & 0.088 & 0.058 & 148 \\
\hline & 3 & 1 & 0.36 & & & & & & & & & & 0.39 & & 0.092 & 0.026 & 165 \\
\hline & 3 & 1 & 46 & & & & & & & 2.65 & 0.42 & 0.419 & 0.38 & 4.70 & 0.097 & 0.051 & 171 \\
\hline & 3 & 1 & & & & & & & & 2.61 & 0.31 & 0.412 & 0.36 & 4.69 & 0.098 & 0.017 & 183 \\
\hline & 3 & 1 & & & & & 7. & 27. & 0.052 & 2.46 & 0.31 & 0.391 & 0.45 & 4.52 & 0.098 & 0.012 & 214 \\
\hline DP14420 & 3 & 1 & 0.76 & 11.01 & & 1.90 & 7.40 & 26.6 & 0.055 & 2.47 & 0.39 & 0.399 & 0.47 & 4.51 & 0.086 & 0.025 & 196 \\
\hline
\end{tabular}




\begin{tabular}{|c|c|c|c|c|c|c|c|c|c|c|c|c|c|c|c|c|c|}
\hline Lab ID & ग्ّ & 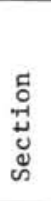 & 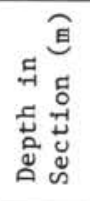 & 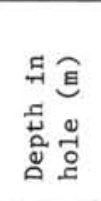 & $\mathrm{Na}$ & $\mathrm{Mg}$ & A1 & S1 & P & K & $\mathrm{Ca}$ & $\mathrm{Ti}$ & Mn & $\mathrm{Fe}$ & $\mathrm{Ba}$ & S & $\mathrm{H}_{2} \mathrm{O}$ \\
\hline 421 & 3 & 1 & .86 & 1 & 0.48 & 1 & 7 & 6 & 53 & 2.61 & 0 & 3 & 0.43 & 7 & 87 & 4 & 150 \\
\hline DP1 4422 & 3 & 1 & .96 & .21 & 0.52 & & & & & & & & & & & & 33 \\
\hline DP1 4423 & 3 & 1 & 1.06 & 11.31 & 0.48 & 1.87 & $7 \cdot 34$ & & & .68 & & & & & & .047 & 133 \\
\hline DP1 4424 & 3 & 1 & 1.16 & 11.41 & 0.49 & 2.02 & 7.64 & & & 2.62 & & & 0. & 4.78 & 8 & 10 & 149 \\
\hline DP1 4425 & 3 & 1 & 1.26 & 11.51 & 0.55 & 2.05 & 7.62 & 26.2 & 49 & 2.69 & 0.44 & 0.427 & 0.49 & 4.81 & 2 & & 132 \\
\hline DP14426 & 3 & 1 & 1.36 & 11.61 & 0.50 & 1.97 & 7.54 & 26.7 & 0.046 & 2.65 & 0.43 & 0.408 & 0.13 & 4.58 & & & 143 \\
\hline DP14427 & 3 & 1 & 1.46 & 11.71 & 0.35 & 2.03 & 7.56 & 26.5 & 0.048 & 2.61 & 0.36 & 0.421 & 0.24 & 4.72 & .092 & & 177 \\
\hline DP1 4428 & 3 & 2 & 0.06 & 11.81 & 0.47 & & 7.58 & 26.2 & 0.046 & 2.64 & 0.37 & 0.415 & 0.33 & & & & 146 \\
\hline DP & 3 & 2 & 0.16 & 11.91 & 0.47 & 2. & 7. & & 9 & & 1 & & & & & & 147 \\
\hline DP1443 & 3 & 2 & 0.26 & 12.01 & 0. & & 7. & & 0 & & & & & & & & 1 \\
\hline DP1 443 & 3 & 2 & 0.36 & 12. & & & & & & & & & & & & & 136 \\
\hline DP1 4432 & 3 & 2 & 0.46 & 12.21 & 0.44 & & 7. & & & & & & & & 6 & 4 & 157 \\
\hline DP1 4433 & 3 & 2 & 0.56 & 12.31 & 0.43 & 1.99 & 7.47 & 26.3 & 0.055 & 2.61 & 0. & 0.4 > & 0.48 & 4.63 & 0. & 0 & 159 \\
\hline DP1 4434 & 3 & 2 & 0.66 & 12.41 & 0.39 & 2.01 & 7.57 & 26.4 & 0.052 & 2.60 & 0.38 & 0.412 & 0.43 & 4.66 & & & 173 \\
\hline DP14435 & 3 & 2 & 0.76 & 12.51 & 0.37 & 1.97 & 7.39 & 26.5 & 0.054 & 2.59 & 0.42 & 0.4 & 0.42 & & & & 184 \\
\hline+36 & 3 & 2 & 0.86 & 12.61 & 0.46 & 1.97 & 7.43 & & 0.0 & & & 0.397 & & & & & 157 \\
\hline DP1 443 & 3 & 2 & 0.96 & 12.71 & 0 . & 2 & & & & & & 5 & & & & & 54 \\
\hline DP14438 & 3 & 2 & 1.06 & 12.81 & 0. & 1 & 7. & & & & & & & & & 5 & 151 \\
\hline DP1 4439 & 3 & 2 & 1.16 & 12.91 & 0.50 & 2.01 & 7.46 & 26.6 & 0.053 & 2.69 & 0.46 & 0.403 & 0.41 & 4.54 & 0 & 1 & 144 \\
\hline DP1 4440 & 3 & 2 & 1.26 & 13.01 & 0.51 & 1.69 & 6.96 & 26.9 & 0.052 & 2.76 & 0.41 & 0.321 & 0.42 & 3.75 & & & 151 \\
\hline DP1 4441 & 3 & 2 & 1.36 & 13.11 & 0.75 & 1.32 & 6.40 & 27.7 & 0.042 & 3.09 & 0.46 & 0.254 & 0.27 & 3. & & & 115 \\
\hline DP1 4442 & 3 & 2 & 1.46 & 13.21 & 0.41 & 2.08 & 7.5 & 26.0 & .050 & 2.70 & 0.43 & 0.409 & 0.38 & & & 1 & 177 \\
\hline DP & 3 & 3 & 0.06 & 13.31 & 0.47 & 2. & 7.2 & 26. & & 2.67 & 0.47 & 0. & 0 . & & & & 175 \\
\hline DP1 & 3 & 3 & 0.16 & 13.41 & 0. & 2. & 7.1 & & 1 & 2.68 & 0 & $0.1+2-1$ & 0 & & & & 9 \\
\hline DP1 444 & 3 & 3 & 0.26 & 13.51 & 0. & 2. & 7. & & & & & & & & & & 198 \\
\hline DP14446 & 3 & 3 & 0.36 & 13.61 & 0 & 2 & 7. & 2 & 0 & 1 & 3 & 0 & b & 5 & & 5 & 204 \\
\hline DP1 4447 & 3 & 3 & 0.46 & 13.71 & 0.42 & 1.99 & 7.51 & 26.6 & 0.054 & 2.58 & 0.38 & 0.407 & 0. & 4.66 & .090 & 2 & 176 \\
\hline 448 & 3 & 3 & 0.56 & 13.81 & 0.40 & 2.09 & 7.49 & 26.1 & 0.052 & 2.67 & 0.47 & 0.416 & 0.2 & 4.69 & 0 & 8 & 177 \\
\hline 149 & 3 & 3 & 0.66 & 13.91 & 0.35 & 2.04 & 7.60 & 26.6 & 0.056 & 2.55 & 0.36 & 0.46 & 0.5 & 4. & 2 & & 204 \\
\hline 450 & 3 & 3 & 0.76 & 14.01 & 0.46 & 2.06 & 7.42 & 26.3 & .05 & 2.61 & 0.49 & 0. & 0. & 4. & & & 76 \\
\hline 1 & 3 & 3 & 0.86 & 14.11 & 0 & & 7. & 8 & & 2.55 & 0. & & 0 . & & & & 174 \\
\hline 452 & 3 & 3 & 0.96 & 14.21 & & & & & & & & & & & & 4 & 169 \\
\hline DP14453 & 3 & 3 & 1.06 & 14.31 & 0.36 & 2. & & & 0 & 2 & 0.36 & 0.405 & 0.39 & 4 & 3 & 36 & 188 \\
\hline DP14454 & 3 & 3 & 1.16 & 14.41 & 0.38 & 2.03 & 7.33 & 26.7 & 0.053 & 2.54 & 0.47 & 0.389 & 0.38 & 4.51 & .093 & 32 & 204 \\
\hline DP1 4455 & 3 & 3 & 1.26 & 14.51 & 0.38 & 2.01 & 7.47 & 27.0 & 0.052 & 2.51 & 0.41 & 0.402 & 0.39 & 4.63 & 0.089 & 0. & 192 \\
\hline DP14456 & 3 & 3 & 1.36 & 14.61 & 0.55 & 2.00 & 7.33 & 26.5 & 0.05 & 2.59 & 0.55 & 0.406 & 0.40 & 4.68 & 0.0 & & 148 \\
\hline P1 4457 & 3 & 3 & 1.46 & 14.71 & 0.45 & 2.03 & 7.51 & 26.6 & 0.0 & 2.63 & 0.41 & 0.413 & 0.33 & 4. & 4 & & 4 \\
\hline 58 & 3 & 4 & 0.06 & 14.81 & 0.41 & 2.06 & 7.54 & 26. & .0 & 2.64 & 0.4 & 0. & 0. & & & & 68 \\
\hline 9 & 3 & 4 & 0.16 & 14.91 & 0 & 2. & 7. & 2 & 3 & 2.59 & 0. & & 0 & & 0 & 27 & 180 \\
\hline DP & 3 & 4 & 0.26 & 15 & & & & & & & & & & & & & 167 \\
\hline DP1 4461 & 3 & 4 & 0.36 & 15.11 & 0.38 & 2. & 7 & 26.8 & 0 . & 2.54 & 0 . & 0. & 0 . & 4 & 2 & 9 & 186 \\
\hline DP14466 & 3 & 4 & 0.46 & 15.21 & 0.37 & 2.11 & 7.52 & 26.3 & 0.056 & 2.59 & 0.44 & 0.404 & 0.37 & 4.61 & 0.097 & 6 & 173 \\
\hline DP1 4462 & 3 & 4 & 0.56 & 15.31 & 0.44 & 2.16 & 7.60 & 26.4 & 0.055 & 2.62 & 0.44 & 0.407 & 0.40 & 4.66 & 0.1 & 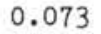 & 181 \\
\hline 14463 & 3 & 4 & 0.66 & 15.41 & 0.40 & 2.09 & 7.66 & 26.5 & 0.055 & 2.60 & 0.39 & 0.4 & 0 . & 4. & 0.0 & & 210 \\
\hline P14464 & 3 & 4 & 0.76 & 15.51 & 0.35 & 2.07 & 7.4 & 26.4 & 0.056 & 2.50 & 0.42 & 0.403 & 0.2 & 4.52 & 0.0 & 6 & 20 \\
\hline 14465 & 3 & 4 & 0.86 & 15.61 & 0.37 & 2.09 & 7.58 & 26.5 & 0.054 & 2.55 & 0.41 & 0.408 & 0.3 & 4. & 5 & & 8 \\
\hline & 3 & 4 & 96 & 1 & & 2.10 & 7.6 & 26.6 & 0.0 & 2.55 & 0. & 0. & & & & & 182 \\
\hline 68 & 3 & 4 & 1 & 1 & 0 & & & & & & & & 5 & 4.63 & 14 & 0. & 188 \\
\hline DP1 4469 & 3 & 4 & 1.16 & 15.91 & 0.37 & 2.06 & 7.48 & 26.2 & 0.057 & 2.49 & 0.43 & 0.402 & 0.45 & 4.58 & 0.095 & 0.038 & 124 \\
\hline DP1 4470 & 3 & 4 & 1.26 & 16.01 & 0.54 & 2.16 & 7.56 & 26.3 & 0.049 & 2.67 & 0.51 & 0.429 & 0.3 & 4.72 & 0.0 & פט & 170 \\
\hline DP14471 & 3 & 4 & 1.36 & 16.11 & 0.40 & 2.11 & 7.62 & 26.4 & 0.053 & 2.58 & 0.42 & 0.416 & 0.41 & 4.70 & 0.091 & +2 & 171 \\
\hline P14472 & 3 & 4 & 1.46 & 16.21 & 0.58 & 2.14 & 7.6 & 26.2 & 0.055 & 2.70 & 0.51 & 0.426 & 0.38 & 4.74 & 0.095 & 33 & 164 \\
\hline P14473 & 3 & 5 & 0.06 & 16.31 & 0.43 & 2.12 & 7.62 & 26.3 & 0.054 & 2.62 & 0.41 & 0.417 & 0.1 & 4.68 & 0.0 & & 163 \\
\hline 174 & 3 & 5 & 0.16 & 1 & 0.52 & 2.17 & $7.68 \mathrm{c}-\mathrm{c}$ & 26.3 & 0.058 & 2.65 & 0.51 & 3 & & & & & 75 \\
\hline DP1 & 3 & 5 & -5 & & & 2.1 & 7 & 5 & 9 & 7 & & & & & & & 162 \\
\hline 476 & 3 & C & 0.06 & 63 & 0.48 & 2.18 & 7.5 & 26.2 & 0.056 & & 0.54 & 0.421 & 0.48 & 4.71 & 0.090 & & 182 \\
\hline & 3 & C & 0.16 & 16.73 & 0.38 & 2.16 & 7.64 & 26.4 & 56 & 2.49 & 0.44 & 0.411 & 0.46 & 4.69 & 0.093 & 0.041 & 182 \\
\hline P14478 & 3 & C & 0.26 & 16.83 & 0.61 & 2.15 & 7.61 & 26.2 & 0.049 & 2.80 & 0.47 & 0.429 & 0.30 & 4.75 & 0.095 & 0.104 & 156 \\
\hline P1 4479 & 4 & 1 & 0.06 & & 0.47 & 2.26 & 7.68 & 26.2 & 0.053 & 2.33 & 0.40 & 0.396 & 0.39 & 4.39 & 0.086 & 0.035 & \\
\hline PP1 4480 & 4 & 1 & 0.16 & & 0.36 & 2.14 & 7.8 & 26.5 & 0.060 & 2.57 & 0.37 & 0.429 & 0.55 & 4.75 & 0.100 & & \\
\hline P1 4481 & 4 & 1 & 0.26 & & 0.42 & 2.10 & 7.65 & 26.1 & 0.060 & 2.50 & 0.42 & 0.414 & 0.51 & 4.72 & 0.093 & 0.031 & \\
\hline 82 & 4 & 1 & 0.76 & 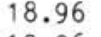 & 0.45 & 2.17 & 7.79 & 26.4 & 0.0 & 2.62 & 0. & 0. & 0.49 & 4.81 & & 0. & 168 \\
\hline DP1 4483 & 4 & 1 & 0.86 & 19.06 & 0.38 & 2.12 & 7.65 & 25.7 & 0.058 & 2.38 & 0.51 & 0.406 & 0.56 & 4.82 & 0.089 & 0.037 & 195 \\
\hline
\end{tabular}


Table 2. (Continued).

\begin{tabular}{|c|c|c|c|c|c|c|c|c|c|c|c|c|c|c|c|c|c|}
\hline Lab ID & ஸ̆ & 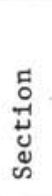 & 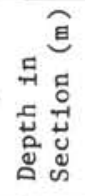 & 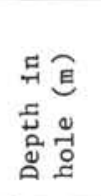 & $\mathrm{Na}$ & $\mathrm{Mg}$ & Al & Si & $\mathrm{P}$ & K & $\mathrm{Ca}$ & $\mathrm{Ti}$ & Mn & $\mathrm{Fe}$ & $\mathrm{Ba}$ & S & $\mathrm{H}_{2} \mathrm{O}$ \\
\hline P14484 & 4 & 1 & 0.96 & 16 & 0.40 & 15 & 7.87 & 2 & 0.058 & 2.43 & 0.52 & 28 & 0.55 & 4.93 & 0.088 & 0.031 & 184 \\
\hline DP14485 & 4 & 1 & .06 & 19.26 & 0.43 & 2 & 7.76 & & 59 & 2.45 & & 10 & .55 & & 0.088 & & 173 \\
\hline DP14486 & 4 & 1 & 1.16 & 19.36 & 0.44 & 2.11 & 7 & & 0 & 2 & & & & 4.80 & 0.088 & 0.038 & 175 \\
\hline DP1 4487 & 4 & 1 & 1.26 & 19.46 & 0.40 & 2.12 & 7.88 & 26.3 & 0.058 & 2.59 & 0.44 & 0.439 & 0.57 & 4.94 & 0.088 & 0.038 & 173 \\
\hline DP1 4488 & 4 & 1 & 1.36 & 19.56 & 0.42 & 2.09 & 7.74 & 25.9 & 0.055 & 2.57 & 0.44 & 0.422 & 0.54 & 4.88 & 0.088 & 0.042 & 171 \\
\hline DP1 4489 & 4 & 1 & 1.46 & 19.66 & 0.47 & 2.11 & 7.82 & 26.0 & 0.056 & 2.60 & 0.44 & 0.429 & 0.57 & 4.94 & 0.092 & 0.045 & 159 \\
\hline DP1 4490 & 4 & 2 & 0.05 & 19.75 & 0.41 & 2.10 & 7.85 & 26.0 & 0.056 & 2.61 & 0.46 & 0.424 & 0.55 & 4.91 & & 0.042 & 164 \\
\hline DP1 4491 & 4 & 2 & 0.16 & & 0.44 & 2.10 & 7.80 & 25.9 & 0.058 & 2.58 & 0.44 & 0.425 & 0.55 & 4.87 & & 0.048 & 166 \\
\hline DP14492 & 4 & 2 & 0.26 & 19.96 & 0.43 & 2.10 & 7.82 & 26.0 & 0.056 & 2.62 & 0.41 & 27 & & & 92 & & 155 \\
\hline P14493 & 4 & 2 & 0.36 & 20.06 & 0.45 & 2.07 & 7.81 & 2 & 0 & 2.61 & & 1 & & & 99 & & 155 \\
\hline P14494 & 4 & 2 & 0.46 & 20.16 & 0. & 2. & 7. & 26 & 0 & 2 & & 1 & 0 & & 91 & 4 & 148 \\
\hline P1 4495 & 4 & 2 & 0.56 & 20.26 & 0.45 & 2.06 & 7.79 & 25.7 & 0.055 & 2.58 & 0.45 & 0.424 & 0.55 & 4.93 & 0.088 & 0.049 & 160 \\
\hline DP1 4496 & 4 & 2 & 0.66 & 20.36 & 0.47 & 2.11 & 7.92 & 26.0 & 0.058 & 2.61 & 0.46 & 0.432 & 0.58 & 5.00 & 0.087 & 0.064 & 165 \\
\hline DP1 4497 & 4 & 2 & 0.76 & 20.46 & 0.43 & 2.07 & 7.83 & 25.6 & 0.058 & 2.57 & 0.45 & 0.422 & 0.59 & 4.95 & 0. & 0. & 159 \\
\hline DP14498 & 4 & 2 & 0.86 & 20.56 & 0.47 & 2.06 & 7.92 & 25.8 & 0.060 & 2.61 & 0.47 & 0.434 & & & & & 153 \\
\hline DP1 4499 & 4 & 2 & 0.96 & 20.66 & 0.45 & 2.02 & 7.83 & 25.5 & 0.060 & 2.57 & 0.46 & 0.424 & 0.61 & 4.97 & & & 160 \\
\hline DP1 4500 & 4 & 2 & 1.06 & 20.76 & 0.53 & 2.01 & 7.92 & 25.7 & 0.059 & & 0.49 & & 1 & & 2 & 6 & 144 \\
\hline 001 & 4 & 2 & 1.16 & 20.86 & 0.47 & 2.01 & 7.93 & 2 & 0 & 2 & & 2 & 0 & & 3 & 8 & 48 \\
\hline P14502 & 4 & 2 & 1.26 & 20.96 & 0.47 & 1. & 7. & 25 & 0 & 2.66 & 0.43 & 0.426 & 0.59 & 4.93 & 8 & 7 & 150 \\
\hline P14503 & 4 & 2 & 1.36 & 21.06 & 0.44 & 2.00 & 7.72 & 25.3 & 0.057 & 2.60 & 0.43 & 0.418 & 0.62 & 4.89 & 0 . & 0.053 & 157 \\
\hline DP14504 & 4 & 2 & 1.46 & 21.16 & 0.50 & 2.02 & 7.84 & 25.7 & 0.059 & 2.63 & 0.45 & 0.432 & 0.62 & 4.97 & & 0.054 & 152 \\
\hline DP1 4505 & 4 & 3 & 0.06 & 21.26 & 0.51 & 2.01 & 7.59 & 25.0 & 0.058 & 2.56 & 0.47 & 0.412 & 0. & 4.89 & & & 148 \\
\hline DP14506 & 4 & 3 & 0.16 & 21.36 & 0.47 & 2.05 & & 25.5 & 0.0 & & 0 & 6 & 7 & & & & 149 \\
\hline DP1 & 4 & 3 & 0.26 & 21.46 & 0.49 & 2.0 & 7.82 & 25.6 & 3 & 2 & 6 & 6 & 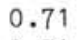 & & 5 & 9 & 155 \\
\hline P14508 & 4 & 3 & 0.36 & 21. & 0. & 2.07 & 7.78 & 25 & 0 & 2 & 4 & 7 & 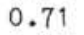 & & 5 & 7 & 152 \\
\hline P1 4509 & 4 & 3 & 0.46 & 21.66 & 0.53 & 2.05 & 7.83 & 25.5 & 0.058 & 2.63 & 0.45 & 0.426 & 0.67 & 5.01 & 0.0 & 8 & 146 \\
\hline DP14510 & 4 & 3 & 0.56 & 21.76 & 0.48 & 2.05 & 7.85 & 25.6 & 0.054 & 2.63 & 0.45 & 0.434 & 0.67 & E $>$ & & 56 & 148 \\
\hline DP1 4511 & 4 & 3 & 0.66 & 21.86 & 0.50 & 2.03 & 7. & 25.5 & 0.053 & 2.5 & 0.49 & 0.429 & & & 9 & & 151 \\
\hline DP14512 & 4 & 3 & 0.76 & 21.96 & 0.50 & 2.03 & & 25.4 & 0.053 & 2.57 & & 4 & & & & & 141 \\
\hline 13 & 4 & 3 & 0.86 & 22.06 & 0.46 & 2.02 & 7. & 25.5 & 0.059 & 2.63 & 2 & 8 & 0 & & & 15 & 44 \\
\hline DP1 & 4 & 3 & 0.96 & 22. & 0. & 2. & 7. & 2 & 1 & 2 & 4 & 7 & 6 & & 6 & & 44 \\
\hline DP1 4515 & 4 & 3 & 1.06 & 22. & 0. & 2. & 7. & 2 & 1 & 2 & 0. & 3 & 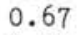 & 8 & 6 & 2 & 146 \\
\hline DP14516 & 4 & 3 & 1.16 & 22.36 & 0.49 & 2.06 & 7.99 & 26.0 & 0.059 & 2.69 & 0.43 & 0.447 & 0.71 & 5.08 & 0.088 & 47 & 140 \\
\hline DP1 4517 & 4 & 3 & 1.26 & 22.46 & 0.47 & 2.04 & 7.88 & 25.6 & 0.061 & 2.66 & 0.43 & 0.442 & 0.68 & 5.02 & 0.0 & 63 & 146 \\
\hline DP14518 & 4 & 3 & 1.36 & 22.56 & 0.44 & 2.04 & 7.86 & 25.4 & .060 & 2.63 & 0.42 & 0.439 & 0.6 & 5. & 5 & 4 & 154 \\
\hline DP1 4519 & 4 & 3 & 1.46 & 22.66 & 0.4 & 2.05 & 7.87 & 25.5 & 0.059 & 2.57 & 0.41 & 9 & 0 . & & & & 158 \\
\hline DP1 & 4 & 4 & 0.06 & 22.76 & 0. & 2.04 & 7.85 & 25.6 & 0.060 & 2.65 & 0.43 & 3 & 8 & & & & 146 \\
\hline DP1 4521 & 4 & 4 & 0.16 & 22.86 & 0 . & 2. & & 2 & 0.063 & 2.65 & 0.43 & 4 & 4 & & 86 & 65 & 143 \\
\hline DP1 4522 & 4 & 4 & 0.26 & 22.96 & 0. & 2.01 & 7. & 2 & 0.065 & 2.65 & 4 & 3 & 3 & 6 & 35 & 1 & 143 \\
\hline DP1 4523 & 4 & 4 & 0.36 & 23.06 & 0.48 & 2.03 & 7.81 & 25.3 & 0.064 & 2.66 & 0.44 & 32 & 0 & 4. & 0 . & 6 & 138 \\
\hline DP1 4524 & 4 & 4 & 0.46 & 23.16 & 0.49 & 2.02 & 7.80 & 25.4 & 0.065 & 2.70 & 0.43 & 0.435 & 0.70 & 4.98 & 34 & 59 & 134 \\
\hline DP1 4525 & 4 & 4 & 0.56 & 23.26 & 0.48 & 2.02 & 7.78 & 25.4 & 0.063 & 2.70 & 0.42 & 0.440 & 0.68 & 4.97 & 0.084 & 0.068 & 135 \\
\hline P14526 & 4 & 4 & 0.66 & 23.36 & 0.53 & 2.01 & 7.75 & 25.2 & 0.061 & 2.72 & 0.41 & 0.437 & 0 . & 4. & 37 & 74 & 127 \\
\hline P1 4527 & 4 & 4 & 0.76 & 23.46 & 0.51 & 1.98 & 7.75 & 25.2 & 0.058 & 2.72 & 0.40 & 0.432 & 0. & 4. & 0. & 0 . & 131 \\
\hline 28 & 4 & 4 & 0.86 & .56 & 0.53 & 1.98 & 7.87 & 25.4 & 0.063 & 2.74 & 0 . & & 4 & & 83 & & 127 \\
\hline DP & 4. & 4 & 0.96 & 6 & 0.5 & 1.99 & 7.85 & 25.3 & 0.0 & 2.73 & 0.43 & & & & & & 134 \\
\hline DP1 4530 & 4 & 4 & 1.06 & 23.76 & 0.47 & 1.96 & 7.81 & 25 & 0.069 & 2.70 & 0.43 & 32 & 9 & 1 & 9 & 6 & 31 \\
\hline DP1 4531 & 4 & 4 & 1.16 & 23.86 & 0.47 & 1.98 & 7.84 & 25.3 & 0.067 & 2.71 & 0.42 & 0.437 & 0.64 & 4.92 & 0.079 & 0.073 & 138 \\
\hline P1 4532 & 4 & 4 & 1.26 & 23.96 & 0.45 & 1.98 & 7.86 & 25.4 & 0.069 & 2.69 & 0.43 & 0.434 & 0.65 & 4.94 & 0.080 & 0.064 & 140 \\
\hline P1 4533 & 4 & 4 & 1.36 & 24.06 & 0.45 & 1.97 & 7.85 & 25.4 & 0.064 & 2.69 & 0.41 & 0.437 & 0.65 & 4.97 & 0.079 & 0.056 & 135 \\
\hline P14534 & 4 & 4 & 1.46 & 4.16 & 0.48 & 1.98 & 7.93 & 25.6 & 0.063 & 2.67 & 0.45 & 0.448 & 0.6 & 7 & 0.078 & 65 & 137 \\
\hline 35 & 4 & 5 & 0.06 & 26 & 0.4 & 1.95 & 7. & 25.5 & 0.060 & 2.65 & 0.44 & & 0.6 & & 76 & 49 & 138 \\
\hline DP1 4536 & 4 & 5 & 0.16 & 36 & 0. & 1.98 & 7. & 25.6 & 0.060 & 2.68 & 0.40 & 40 & 0.66 & & & & 139 \\
\hline DP14537 & 4 & 5 & 0.26 & 24.46 & 0.43 & 1.99 & 7.93 & 25.6 & 0.067 & 2.66 & 0.41 & 0.443 & 0.68 & 4.98 & 0.079 & 0.048 & 140 \\
\hline DP1 4538 & 4 & 5 & 0.36 & 24.56 & 0.47 & 2.00 & 7.86 & 25.5 & 0.070 & 2.68 & 0.42 & 0.444 & 0.71 & 4.98 & 0.080 & 0.056 & 133 \\
\hline DP1 4539 & 4 & 5 & 0.46 & 24.66 & 0.49 & 2.01 & 7.91 & 25.6 & 0.072 & 2.69 & 0.43 & 0.451 & 0.72 & 5. & 0.082 & 0.054 & 132 \\
\hline DP1 4540 & 4 & 5 & 0.56 & 24.76 & 0.46 & 2.02 & 7.95 & 25.7 & 0.075 & 2.70 & 0.42 & 0.446 & 0.70 & 5.02 & 0.087 & 0.051 & 136 \\
\hline P1 4541 & 4 & 5 & 0.66 & 24.86 & 0.43 & 1.98 & 7.90 & 25.5 & 0.071 & 2.66 & 0.39 & 0.437 & 0.72 & 7 & 0.081 & 0.036 & 135 \\
\hline P14542 & 4 & 5 & 0.76 & 24.96 & 0.44 & 1.99 & 7.87 & 25.4 & 0.071 & 2.68 & 0.41 & & 0.71 & & & 0.049 & 134 \\
\hline DP1 4543 & 4 & 5 & 0.86 & 25.06 & 0.41 & 2.00 & 7.96 & 25.6 & 0.072 & 2.71 & 0.40 & 0.442 & 0.75 & 4.99 & 0.077 & 0.049 & 141 \\
\hline P14544 & 4 & 5 & 0.96 & 25.16 & 0.41 & 2.00 & 7.94 & 25. & 0.071 & 2.71 & 0.38 & 0.440 & 0.72 & 4.97 & 0.081 & 0.053 & 143 \\
\hline & 4 & 5 & 1.00 & 25.20 & 0.45 & 1. & 7.89 & 25. & 0.067 & 2.70 & 0.36 & 0.430 & 0. & 4.92 & 0.077 & 0.049 & 134 \\
\hline DP1 4546 & 4 & 5 & 1.16 & 25.36 & 0.45 & 2.01 & & 25.4 & .070 & 2.72 & 40 & 0.434 & 0.12 & 4.91 & 0.078 & 0.069 & 137 \\
\hline
\end{tabular}


Table 2. (Continued).

\begin{tabular}{|c|c|c|c|c|c|c|c|c|c|c|c|c|c|c|c|c|c|}
\hline Lab ID & 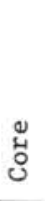 & $\begin{array}{l}\text { 吕 } \\
\text { İ } \\
0 \\
0 \\
\infty\end{array}$ & 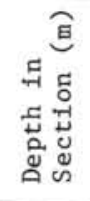 & 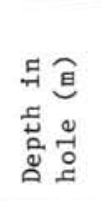 & $\mathrm{Na}$ & $\mathrm{Mg}$ & $\mathrm{Al}$ & $\mathrm{Si}$ & $\mathrm{P}$ & K & $\mathrm{Ca}$ & $\mathrm{T} i$ & Mn & $\mathrm{Fe}$ & $\mathrm{Ba}$ & $\mathrm{S}$ & $\mathrm{H}_{2} \mathrm{O}$ \\
\hline DP1 4547 & 4 & 5 & 26 & 46 & .46 & .01 & 4 & 4 & 067 & 2.73 & 39 & 7 & .71 & 4.93 & 0.080 & .063 & 129 \\
\hline DP1 4548 & 4 & 5 & .36 & & .49 & 00 & 7 & & & 80 & & & 54 & & & & 121 \\
\hline DP14549 & 4 & 5 & 1.46 & & 0.50 & 1.99 & 7.81 & & & 3 & & & & & 0.079 & 0.062 & 117 \\
\hline DP1 4550 & 4 & 6 & 0.06 & 25.76 & 0.51 & 2.01 & 7.89 & 25.7 & 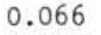 & 2.83 & 0.39 & & 0.67 & 5.05 & 0.080 & .053 & 117 \\
\hline DP1 4551 & 4 & 6 & 0.16 & 25.86 & 0.53 & 2.00 & 7.78 & 25.4 & 0.069 & 2.83 & 0.40 & 0.446 & 0.61 & 5.02 & 0.079 & 0.063 & 110 \\
\hline DP1 4552 & 4 & 6 & 0.26 & 25.96 & 0.51 & 2.01 & 7.83 & 25.6 & 0.073 & 2.83 & 0.39 & 0.451 & 0.61 & & 0.076 & .057 & 116 \\
\hline 553 & 4 & 6 & 0.36 & 26.06 & 0.45 & 2.03 & 7.8 & 25.7 & 0.079 & 2.81 & & 0.456 & 0.69 & & 0. & 0.045 & 117 \\
\hline 54 & 4 & 6 & 0.46 & 26 & 0.51 & 3 & 7. & 25.5 & 4 & & & 0.468 & & & & & 109 \\
\hline DP1 4555 & 4 & 6 & 0.56 & & 0. & & 7. & & & & 0.52 & 2 & & & & & 15 \\
\hline DP14556 & 4 & 6 & 0.66 & 26. & 0 & & 7. & 2 & & 7 & 9 & 2 & & & & & \\
\hline DP1 4557 & 4 & 6 & 0.76 & 26.46 & 0.47 & & 7. & & & & & & & & & & 124 \\
\hline DP1 4558 & 4 & 6 & 0.86 & 26.56 & 0.46 & 2.14 & 7.34 & 25.1 & 131 & 2.76 & 0. & 0.3 & . & & 0.106 & & 123 \\
\hline DP1 4559 & 4 & 6 & 0.96 & 26.66 & 0.46 & 2.17 & 7.28 & 25.1 & 0.174 & 2.76 & 0.64 & 0.523 & 0.99 & & 0.115 & & 129 \\
\hline DP1 4560 & 4 & 6 & 1.06 & 26.76 & 0.45 & 2.18 & 7.18 & 24.9 & 0.173 & 2.73 & 0.64 & 0.511 & 0.98 & & & & 131 \\
\hline DP1 4561 & 4 & 6 & 1.16 & 26.86 & 0.46 & 2.17 & 7.19 & 24. & & 2.74 & 0.64 & 0.514 & & & 0.1 & 7 & 127 \\
\hline DP1 4562 & 4 & 6 & 1.26 & 26.96 & 0.44 & 2.18 & 7.23 & 25. & 0.1 & 2.74 & 4 & 0.5 & & & 0 . & & 128 \\
\hline 563 & 4 & 6 & 1.36 & 27.06 & 0.48 & 2.19 & 7. & & 3 & 2. & & 5 & & & & 0 & 129 \\
\hline 4564 & 4 & 6 & 1.46 & 27.16 & 0. & 2. & 7. & 2 & 3 & 2 & & 7 & 6 & 2 & 2 & 9 & 32 \\
\hline DP1 4565 & 4 & 7 & 0.06 & 27.26 & 0.45 & 2.17 & 7.20 & 24.9 & 0.189 & 2.75 & 0. & 0.5 & 0. & 5. & 7 & 6 & 128 \\
\hline DP1 4566 & 4 & 7 & 0.16 & 27.36 & 0.44 & 2.17 & 7.27 & 25.1 & 0.186 & 2.76 & 0.68 & 0.527 & 1.01 & 5.59 & 6 & & 130 \\
\hline 4567 & 4 & 7 & 0.26 & 27.46 & 0.47 & 2.17 & 7.25 & 24.9 & 0.181 & 2.76 & 0.68 & 0.523 & 1.01 & 3. & & & 129 \\
\hline 1568 & 4 & 7 & 0.36 & 27.56 & 0.49 & 2.19 & 7.21 & 24.9 & 0.193 & 2.77 & 0.73 & 0.532 & S & & & & 127 \\
\hline DP1 4569 & 4 & 7 & 0.46 & 27.66 & 0.45 & 2.15 & 7. & 24.6 & 0. & 2.73 & & 4 & & & & & 128 \\
\hline DP1 4570 & 5 & 1 & 0.36 & & 0.50 & 2.03 & 8.04 & 25.5 & 0.0 & 2.81 & 1 & 0.4 & & & 9 & & \\
\hline 571 & 5 & 1 & 0.46 & 28. & 0. & 2. & 8. & 2 & 0 & 2.76 & & & & & & & 119 \\
\hline 572 & 5 & 1 & 0.56 & 28. & 0. & 2 & 7. & 2 & 9 & 2 & & 0 & 1 & : & 9 & 9 & 121 \\
\hline DP1 4573 & 5 & 1 & 0.66 & 28.26 & 0.46 & 2.04 & 7.93 & 25.1 & 0.082 & 2.72 & 4 & 0.446 & 0.92 & 5 . & .079 & 0. & 121 \\
\hline DP1 4574 & 5 & 1 & 0.76 & 28.36 & 0.46 & 2.05 & 7.84 & 24.9 & 0.085 & 2.69 & 0.45 & 0.447 & 0.8 & 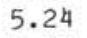 & 0 & & 126 \\
\hline 4575 & 5 & 1 & 0.86 & 28.46 & 0.47 & 2.06 & 7.84 & 25.0 & 0.084 & 2.68 & 0.47 & 0.4 & & & & & 121 \\
\hline DP1 4576 & 5 & 1 & 0.96 & 28.56 & 0.44 & 2.08 & 7.83 & 25.0 & 0.086 & 2.67 & 0 . & 7 & 0 & & 2 & 1 & 127 \\
\hline DP1 4577 & 5 & 1 & 1.06 & 28.66 & 0.46 & 2.07 & 7.86 & 25.0 & 0.083 & 2.69 & 0.46 & +9 & & & 31 & & 132 \\
\hline 78 & 5 & 1 & 1.16 & 28. & 0 & 2 & 7. & 21 & 4 & 2.63 & 6 & 1 & 3 & & 7 & 7 & 135 \\
\hline DP & 5 & 1 & 1.26 & 28. & 0 & 2 & 7. & 2 & 6 & 69 & 6 & 19 & 1 & 3 & 0.077 & 0.060 & 136 \\
\hline 580 & 5 & 1 & 1.36 & 28 & 6 & 2. & 7. & 2 & 5 & 1 & 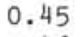 & 0.438 & 0. & 5 & 0.079 & 0.059 & 138 \\
\hline 581 & 5 & 1 & 1.46 & 29.06 & .51 & 2.07 & 7.81 & 24.9 & 0.086 & 2.72 & 0.46 & 0.440 & 0.9 & 5. & .077 & 0. & 133 \\
\hline 582 & 5 & 2 & 0.56 & & 0.48 & 2.07 & 7.90 & 25.2 & 0.084 & 2.71 & .47 & 0.460 & 0.8 & 5. & 0 & 0.0 & \\
\hline DP1 4583 & 5 & 2 & 1.16 & & 0.45 & 2.0 & 7.75 & 25.0 & 0.09 & 2.7 & 0.44 & 0.451 & 0. & 22 & 0. & & \\
\hline 4584 & 5 & 3 & 0.23 & & .51 & 2.0 & 7. & 24.7 & 0 & 2.77 & 0.50 & 5 & & & & & \\
\hline & 5 & 3 & 0.99 & & & 4 & 7. & 2 & 0 & 82 & 0. & & 4 & 5 & 76 & 9 & \\
\hline 86 & 5 & 4 & 0.76 & & & 1. & 7. & & & 8 & & & & 9 & 5 & 0 . & \\
\hline DP1 4587 & 5 & 5 & 0.46 & & 55 & 2 & 7. & 24.4 & 5 & 2 & 5 & 0.1 & 1. & 1 & 0.071 & 0.071 & \\
\hline DP1 4588 & 5 & 5 & 0.56 & 31.06 & 0.54 & 2.06 & 7.88 & 24.0 & 0.150 & 2.65 & 0.61 & 0.444 & 1.49 & 5.37 & 0.074 & 0.073 & 134 \\
\hline DP1 4589 & 5 & 5 & 0.66 & 31.16 & 0.51 & 2.06 & 7.89 & 23.8 & 0.156 & 2.63 & 0.61 & 0.447 & 1.56 & 5.37 & 0. & 0. & 134 \\
\hline 4590 & 5 & 5 & 0.76 & 31.26 & 0.57 & 2.02 & 7.93 & 24.0 & 0.155 & 2.72 & 0.61 & 0.446 & 1.47 & 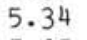 & 0.0 & & 129 \\
\hline 591 & 5 & 5 & 0.86 & 31.36 & 0.65 & 1.91 & 7. & 23.6 & 0.151 & 2.73 & 0.61 & 0.418 & 1.40 & 5 & 0.067 & 0 . & 139 \\
\hline 92 & 5 & 5 & 0.96 & 31.46 & 0.54 & 2.02 & 7. & 23.8 & 0.172 & 2.60 & 0.67 & & & & & & 146 \\
\hline 93 & 5 & 5 & & & & & 7. & 24.0 & & 2.61 & 0.71 & & & & & 0. & 146 \\
\hline & 5 & 5 & 1 & & & & & & & 2.66 & & & & & 4 & 0.064 & 143 \\
\hline DP14 & 5 & 5 & & & 0.53 & 2 & 7. & 8 & 0 & 2.67 & 0 & 0. & 1.70 & 5. & 0.074 & 0. & 136 \\
\hline DP14596 & 5 & 5 & 1.46 & 31.96 & 0.6 & 1. & 7. & 23.8 & 0.1 & 2.69 & 0 . & 0.445 & 1.67 & 5.29 & 0.072 & 0. & 134 \\
\hline DP1 4597 & 5 & 6 & 0.06 & 32.06 & 0.64 & 2.01 & 7. & 23.7 & 0.197 & 2.65 & 0.78 & 0.447 & 1.84 & 5.32 & 0.072 & 0.070 & 139 \\
\hline DP1 4598 & 5 & 6 & 0.16 & 32.16 & 0.63 & 2.0 & 7. & 23.8 & 0.202 & 2.64 & 0.78 & 0.452 & 1.65 & 5.38 & 0.072 & 0.071 & 145 \\
\hline DP1 4599 & 5 & 6 & 0.26 & 32. & 0.61 & 2.0 & 7.69 & 23.9 & 0.187 & 2.63 & 0.73 & 0.456 & 1.58 & 4 & 0.074 & 0. & 137 \\
\hline DP1 4600 & 5 & 6 & 0.36 & 32.36 & 0.6 & 2.01 & 7.63 & 23.8 & 0.172 & 2.63 & 0.69 & 0.455 & 1.53 & 5.45 & 0.073 & & 140 \\
\hline DP1 4601 & 5 & 6 & 0.46 & 32.46 & 0.60 & 2.00 & 7.61 & 23.7 & 0.174 & 2.62 & 0.69 & 0.446 & 1.58 & 5.43 & 0.070 & & 142 \\
\hline DP1 4602 & 5 & 6 & 0.56 & 32.56 & 0.60 & 1.99 & 7.73 & 23.8 & 0.176 & 2.66 & & & & & 0.073 & & 143 \\
\hline DP1 4603 & 5 & 6 & 0.66 & 32.66 & 0.59 & 1.98 & 7.72 & 23.7 & 0.169 & 2.68 & 0.67 & 0.450 & 1.56 & 45 & 0.071 & 0.063 & 143 \\
\hline DP1 4604 & 5 & 6 & 0.76 & 32.76 & 0.59 & 1.99 & 7.83 & 23.9 & 0.173 & 2.74 & 0.68 & 0.446 & 1.53 & 5.41 & 0.072 & 0.060 & 141 \\
\hline 4605 & 5 & 6 & 0.86 & & 0.58 & 1.99 & 7.87 & 24.0 & & 2.79 & 0.66 & 0.449 & 1.51 & 5.36 & 0.074 & 0.065 & 136 \\
\hline DP1 4606 & 5 & 6 & 0.96 & 32.96 & 0.5 & 1.98 & 7.88 & 24.0 & 0.169 & 2.81 & 0.67 & 0.445 & 1.48 & & 0.074 & 0.062 & 133 \\
\hline DP1 4607 & 5 & 6 & 1.07 & 33.07 & 0.60 & 1.94 & 7.76 & 23.6 & 0.180 & 2.76 & 0.68 & 0.428 & 1.45 & & 0.071 & 0.059 & 139 \\
\hline DP1 4608 & 5 & 6 & 1.16 & 33.16 & 0.57 & 1.97 & 7.86 & 23.9 & 0.196 & 2.74 & 0.73 & 0.453 & 1.64 & 5.43 & 0.074 & & 145 \\
\hline DP1 4609 & 5 & 6 & 1.26 & 33.26 & 0.55 & 1.95 & 7.74 & 23.6 & 0.201 & 2.68 & 0.73 & 0.438 & 1.65 & 5.39 & 0.074 & 0.060 & 148 \\
\hline
\end{tabular}


Table 2. (Continued).

\begin{tabular}{|c|c|c|c|c|c|c|c|c|c|c|c|c|c|c|c|c|c|}
\hline Lab ID & ్ㅜㅇ & 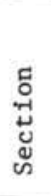 & 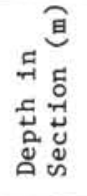 & 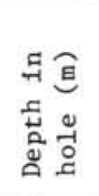 & $\mathrm{Na}$ & $\mathrm{Mg}$ & Al & Si & $\mathrm{P}$ & K & $\mathrm{Ca}$ & Ti & Mn & $\mathrm{Fe}$ & $\mathrm{Ba}$ & $\mathrm{S}$ & $\mathrm{H}_{2} \mathrm{O}$ \\
\hline 510 & 5 & 6 & 35 & & 0.56 & 1.99 & 7.84 & 3.9 & 192 & 2.71 & 0.72 & .458 & 1.74 & 5.53 & 0.074 & 0.061 & 146 \\
\hline & 5 & 6 & .45 & & 0.58 & 1.96 & 7.74 & .5 & 0 & 0 & & .439 & 1.70 & & 0.073 & & 146 \\
\hline DP14612 & 5 & 7 & 0.06 & & 0.61 & 1.96 & 7.73 & 23.5 & 0.200 & 2.70 & 0.76 & .445 & 1.74 & 5.44 & 0.073 & 0.074 & 150 \\
\hline DP14613 & 5 & 7 & 0.16 & & 0.54 & 1.99 & 7.74 & 23.5 & 0.204 & 2.70 & 0.77 & .453 & 1.80 & 5.52 & 0.074 & 0.074 & 152 \\
\hline DP14614 & 5 & 7 & 0.26 & 76 & 0.57 & 2.00 & 7.72 & 23.4 & 0.211 & 2.66 & 0.78 & .452 & 1.78 & 5.53 & 0.074 & 0.084 & 152 \\
\hline DP1 & 5 & 7 & 0.36 & & 0.52 & 2.04 & 7.67 & 23.3 & 0.211 & 2.61 & 0.79 & 0.452 & 1.76 & 5.57 & 0.070 & 0.086 & 156 \\
\hline DP1 & 5 & 7 & 0.45 & 33.95 & 0.47 & 2.04 & 7.79 & 23.7 & 0.222 & 2.60 & 0.79 & .470 & 1.88 & 5.66 & 0.072 & 0.082 & 157 \\
\hline DP1 4617 & 6 & 1 & $1 \cdot 31$ & & & 2. & 7.72 & & 79 & 2.54 & & & 2.09 & 5.33 & 75 & 0.064 & \\
\hline DP1 4618 & 6 & 1 & 1.41 & & & 1. & 7. & & & & & & & & & & \\
\hline DP1 4619 & 6 & 2 & 1.21 & & 0.35 & 2.05 & & 22.0 & & & & 0.442 & 2.14 & 5.48 & 0.076 & 39 & \\
\hline DP1 4620 & 6 & 2 & $1 \cdot 31$ & & 0.39 & 2.10 & 7.38 & 22.6 & 0.545 & 2.55 & 1.02 & 0.432 & 2.25 & 5.54 & 0.075 & 0.110 & \\
\hline DP1 4621 & 6 & 2 & 1.41 & & 0.37 & 2.05 & 7.33 & 22.5 & 0.591 & 2.52 & 1.69 & 0.433 & 2.20 & 5.48 & 0.074 & 0.094 & \\
\hline DP1 4622 & 6 & 3 & 0.06 & & 0.39 & 2.10 & 7.49 & 22.8 & 0.502 & 2.59 & 1.51 & 0.413 & 2.27 & 5.45 & 0.074 & & \\
\hline DP1 & 6 & 3 & 0.16 & & 0 & 2.06 & 7.43 & 22.6 & 0.5 & 2.56 & 1.51 & 0.1 & 2.25 & 5.44 & 0.073 & & \\
\hline 523 & 6 & 3 & 0.26 & & 0. & 2.13 & 7.35 & 22.5 & 0.5 & 2.56 & & 0.403 & 2.21 & 5.38 & 0. & & \\
\hline DP1 4625 & 6 & 3 & 0.36 & & 0 & & 7.56 & 23 & 0 & & & 3 & 9 & 5. & 3 & & \\
\hline DP14626 & 6 & 3 & 0.46 & & 3 & 2 & 7. & & & & & & 2. & 5.29 & 0. & 0 & \\
\hline DP1 4627 & 6 & 3 & 0.56 & & .34 & 2.10 & 7.55 & 22.9 & 0.503 & 2.61 & 1.45 & 0.394 & 2.17 & 5.32 & 0.073 & 0.087 & \\
\hline DP1 4628 & 6 & 3 & 0.66 & & 0.35 & 2.09 & 7.47 & 22.6 & 0.485 & 2.59 & 1.44 & 0.386 & 2.13 & 5.24 & 0.073 & 0.093 & \\
\hline DP14629 & 6 & 3 & 0.76 & 38.06 & 0.37 & 2.06 & 7.98 & 23.9 & 0.313 & 3.01 & 0.88 & 0.379 & 1.50 & & 0.072 & & 122 \\
\hline DP1 4630 & 6 & 3 & 0.86 & 38.16 & 0.35 & 2.05 & 8.01 & 23.9 & 0.3 & 3.01 & 0.88 & 0.378 & 1.51 & 4.99 & 0.073 & 3 & 122 \\
\hline DP14631 & 6 & 3 & 0.96 & 38 & 0.35 & 2.03 & 7. & 23 & 0 & 2 & 5 & 5 & 1. & 4. & & & 124 \\
\hline DP1 4632 & 6 & 3 & 1.06 & 38.36 & 0.38 & 2. & 8. & 23.9 & 0. & 3.01 & 0.86 & 0 . & 1.50 & 4.98 & 0. & 1 & 125 \\
\hline DP1 4633 & 6 & 3 & 1.16 & 38.46 & 0.38 & 2.07 & 8.01 & 24.0 & 0.319 & 3.02 & 0.88 & 0.393 & 1.53 & 5.02 & 0. & 9 & \\
\hline DP14634 & 6 & 3 & 1.26 & 38.56 & 0.34 & 2.07 & 7.97 & 24.0 & 0.289 & 2.96 & 0.82 & 0.384 & 1.53 & 4.98 & 0.072 & 0 . & 125 \\
\hline 635 & 6 & 3 & 1.36 & 66 & 0.37 & 2.06 & 7.95 & 23.8 & 0.297 & 2.92 & 0.86 & 0.3 & 1.66 & 4.96 & 0. & 0. & 123 \\
\hline DP1 4636 & 6 & 3 & 1.46 & .76 & 0.36 & 2.02 & 7.88 & 23.6 & 0.294 & 2.86 & 0.85 & 0 . & 1.70 & 4.91 & 0.073 & 0. & 127 \\
\hline DP14637 & 6 & 4 & 0.06 & 38.86 & 0.34 & 2.11 & 7.84 & 23.5 & 0.3 & 2. & 0.87 & 40 & 1.58 & 5 & 0. & & 123 \\
\hline DP1 4638 & 6 & 4 & 0.16 & 38.96 & 0.33 & 2. & 7. & 23 & 0. & 2. & 0.81 & 0.473 & 1.74 & & 6 & 1 & 119 \\
\hline DP14639 & 6 & 4 & 0.26 & & 0. & 2. & 7. & 2. & 1 & & & & 0 & 3 & 3 & 0 & 121 \\
\hline DP1 4640 & 6 & 4 & 0.36 & & 0. & 2. & 7. & 6 & 0 & 2 & 0 & 0 & 1. & 5 & 2 & 1 & 122 \\
\hline DP14641 & 6 & 4 & 0.46 & 6 & 0. & 2.10 & 7.9 & 23.6 & 0.311 & 2.77 & 0 & 0 & 1.53 & 5. & 2 & 0. & 124 \\
\hline DP1 4642 & 6 & 4 & 0.56 & 36 & 0.39 & 2.04 & 7.91 & 23.4 & 0.360 & 2.64 & 0.99 & .532 & 1.57 & 5.40 & 0.071 & 0. & 129 \\
\hline DP1 4643 & 6 & 4 & 0.66 & .46 & 0.40 & 1.98 & 7.89 & 23.4 & 0.352 & 2.68 & 0.95 & 0.550 & 1.45 & 5.5 & 1 & 0. & 128 \\
\hline DP1 4644 & 6 & 4 & 0.76 & .56 & 0.38 & 1.95 & 7.92 & 23.6 & 0.325 & 2.81 & 0.91 & 0.544 & 1.48 & 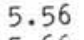 & 0.072 & 0. & 132 \\
\hline DP1 4645 & 6 & 4 & 0.86 & & 0.37 & 2.02 & 7.9 & 23.5 & 0.364 & 2.65 & 0.98 & 0.595 & 1.59 & & 0.073 & 6 & 139 \\
\hline DP1 4646 & 6 & 4 & 0.96 & & 0.34 & 2.01 & 7. & 23.0 & 0.327 & 2.55 & 0.91 & 90 & 1.64 & 5.65 & 75 & 69 & 140 \\
\hline DP1 4647 & 6 & 4 & 1.06 & & 0 & 2 & & 2 & 0 & 2 & 0.94 & 0 . & 1.74 & 5.80 & 0.076 & 65 & 137 \\
\hline DP1 4648 & 6 & 4 & 1.16 & & 0.3 & 2.03 & 7.88 & 23.1 & 0.345 & 2.64 & 0.88 & 0.628 & 1.66 & 5.90 & 0.072 & 0.068 & 138 \\
\hline DP14649 & 6 & 4 & 1.26 & 06 & 0.34 & 2.01 & 7.86 & 23.2 & 0.301 & 2.69 & 0.83 & 0.622 & 1.68 & 5.88 & 0.077 & 0.063 & 140 \\
\hline DP1 4650 & 6 & 4 & 1.36 & .16 & 0.36 & 2.03 & 7.89 & 23.2 & 0.324 & 2.62 & 0.86 & 0.621 & 1.74 & 5.92 & 0.077 & 0. & 140 \\
\hline DP14651 & 6 & 4 & 1.46 & .26 & 0.39 & 2.05 & 7.87 & 23.1 & 0.325 & 2.65 & 0.89 & 11 & 1.74 & 5.95 & 0.078 & 0. & 44 \\
\hline DP1 4652 & 6 & 5 & 0.06 & .36 & 0.4 & 2.03 & 7.7 & 22.7 & 0.319 & 2.63 & 0.89 & 0.583 & 1.66 & 5.85 & 0.075 & & 140 \\
\hline DP1 & 6 & 5 & 0.16 & & 0.4 & 2.04 & 7.79 & .0 & 3 & 2.63 & 8 & & 1.67 & & & & 8 \\
\hline DP1 & 6 & 5 & 0. & & & & & 3 & 4 & 2 & 39 & 08 & 1.68 & 6.01 & 77 & 0. & 39 \\
\hline 655 & 6 & 5 & 0.36 & 40.66 & 0.43 & 2. & 7 . & & 0. & 2.69 & 0.89 & 2 & 1.73 & 6.02 & 0.075 & 0.086 & 141 \\
\hline 4656 & 6 & 5 & 0.46 & .76 & .39 & 2.04 & 7.87 & 23.2 & 0.335 & 2.64 & 0.90 & 0.608 & 1.69 & 6.03 & 0.075 & 0. & 139 \\
\hline DP1 4657 & 6 & 5 & 0.56 & 40.86 & 0.40 & 2.05 & 7.82 & 23.0 & 0.308 & 2.65 & 0.89 & 0.599 & 1.68 & 5.98 & 0.075 & 0.083 & 141 \\
\hline DP1 4658 & 6 & 5 & 0.66 & 40.96 & 0.41 & 2.07 & 7.89 & 23.3 & 0.309 & 2.66 & 0.88 & 0.607 & 1.70 & 6.03 & 0.075 & 0.079 & 137 \\
\hline P14659 & 6 & 5 & 0.76 & 41.06 & 0.4 & 2.07 & 7.86 & 23.2 & 0.301 & 2.66 & 0.86 & 0.598 & 1.67 & 6.02 & 0.076 & & 137 \\
\hline DP1 4660 & 6 & 5 & 0.86 & 41.16 & 0.41 & 2.04 & 7.85 & 23.2 & 0.298 & 2.68 & 0.85 & 0.593 & 1.57 & 5.93 & 0.075 & 0.083 & 137 \\
\hline DP14661 & 6 & 5 & 0.96 & 41.26 & 0.36 & 2.01 & 7.93 & 23.5 & 0.2 & 2.77 & 0.79 & & & 5. & 0. & 0. & 132 \\
\hline & 6 & 5 & 1.06 & $\cdot 36$ & 0.3 & 2 & & 23 & & 2.78 & 0.81 & 5 & 1.34 & 5. & 0.070 & 0.074 & 130 \\
\hline 14663 & 6 & 5 & 1.16 & 41.46 & 0.3 & 2.04 & 7 . & 23 & 0.293 & 2.79 & 0.82 & 0.599 & 1.35 & 5 & 0.070 & 0 & 132 \\
\hline 14664 & 6 & 5 & 1.26 & 41.56 & 0.35 & 2.02 & 7.9 & 23.6 & 0.290 & 2.7 & 0.80 & 0.598 & 1.40 & 5.80 & 0.070 & 0.062 & 130 \\
\hline 14665 & 6 & 5 & 1.36 & 41.66 & 0.3 & 2.05 & 7.86 & 23.3 & 0.299 & 2.7 & 0.80 & 0.615 & 1.38 & & 0.072 & 0.071 & 133 \\
\hline & 6 & 5 & 1.46 & 41.76 & 2.2 & 2.15 & 7.71 & 23. & 0.332 & 2.74 & 0.84 & 0.677 & 1.46 & 6.21 & 0.075 & 0.074 & 138 \\
\hline DP14667 & 6 & 6 & 0.06 & 41.86 & 0.35 & 2.11 & 7.69 & 23.1 & 0.316 & 2.75 & 0.85 & 0.721 & 1.44 & 6.40 & 0.075 & 0.067 & 140 \\
\hline 14668 & 6 & 6 & 0.16 & 41.96 & 0.34 & 2.11 & 7.6 & 23.0 & 0.360 & 2.7 & 0.89 & 0.723 & 1.40 & & & & 140 \\
\hline 114669 & 6 & 6 & 0.26 & 42.06 & 0.37 & 2.10 & 7.66 & 23.0 & 0.319 & 2.74 & 0.87 & 0.727 & 1.40 & & & & 9 \\
\hline P14670 & 6 & 6 & 0.36 & 42.16 & 0.42 & 2.09 & 7.63 & 22.9 & 0.322 & 2.72 & 0.86 & 0.724 & 1.36 & 6.50 & 0.074 & 0.062 & 141 \\
\hline & 6 & 6 & 0.46 & & & 2.0 & 7. & 23 & & 2.8 & 0.87 & 0.748 & 1.36 & & 0.075 & 0.059 & 138 \\
\hline 2 & 6 & 6 & & & & 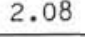 & & 3.5 & 0.311 & 3.03 & .85 & 0.741 & 1.29 & & 0.075 & 0.064 & 130 \\
\hline
\end{tabular}


Table 2. (Continued).

\begin{tabular}{|c|c|c|c|c|c|c|c|c|c|c|c|c|c|c|c|c|c|}
\hline Lab ID & 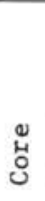 & 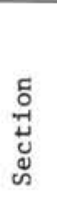 & 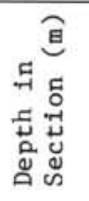 & 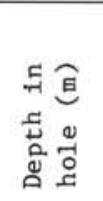 & $\mathrm{Na}$ & $\mathrm{Mg}$ & A1 & S1 & P & $\mathrm{K}$ & $\mathrm{Ca}$ & T1 & Mn & $\mathrm{Fe}$ & $\mathrm{Ba}$ & $\mathrm{S}$ & $\mathrm{H}_{2} \mathrm{O}$ \\
\hline 73 & 6 & 6 & 66 & .46 & 43 & 7 & 76 & 6 & 287 & 2 & 81 & 0.742 & 1.21 & 6.28 & 074 & 7 & 127 \\
\hline & 6 & 6 & 76 & 56 & & & & & & & & & & & & & 25 \\
\hline P14675 & 6 & 6 & .86 & .66 & .40 & .12 & 7.70 & 7 & & 17 & & 30 & .17 & .22 & & & 28 \\
\hline DP1 4676 & 6 & 6 & 0.96 & 42.76 & 0.38 & 2.08 & 7.53 & 3.3 & .248 & .12 & 0.69 & .656 & 1.14 & 5.85 & .072 & & 119 \\
\hline DP1 4677 & 6 & 6 & 1.06 & 42.86 & 0.41 & 2.18 & 7.63 & 3.8 & 0.230 & 20 & 0.65 & .710 & & & & & 115 \\
\hline DP1 4678 & 6 & 6 & 1.16 & 42.96 & 0.37 & 2.17 & 7.73 & 24.0 & & & & & & & & & 115 \\
\hline DP1 4679 & 6 & 6 & 1.26 & 43.06 & 0.34 & 2.17 & 7.72 & & & & & & & & .075 & & 11 \\
\hline DP1 & 6 & 6 & 1.36 & 43.16 & 0.33 & 2.18 & 7.65 & 23.9 & & & & & & & .078 & & 06 \\
\hline DP & 6 & 6 & 1.46 & 43.26 & 0.31 & 2.26 & 7.71 & & 8 & & 9 & 0.593 & & & & & \\
\hline 682 & 6 & 7 & 0.06 & 43.36 & 0. & 3 & 7. & 2 & & & & 3 & & & & & 14 \\
\hline 583 & 6 & 7 & 0.16 & 43.46 & 0.30 & 2.27 & 7.67 & 3.9 & & & & & & & & & 107 \\
\hline & 6 & 7 & 0.26 & 43.56 & 0.32 & 2.24 & 7.68 & 24.1 & 0.260 & & 0. & 0.5 & 1.15 & & 6 & & 109 \\
\hline 85 & 6 & 7 & 0.36 & 43.66 & 0.30 & 2.31 & 7.77 & 24.4 & 0.272 & & 0 & & 1.13 & & & & 105 \\
\hline DP & 6 & 7 & 0.44 & 43.74 & 0.32 & 2.31 & 7.7 & 4.1 & 258 & & & & & & & & 104 \\
\hline DP1 & 7 & 1 & 0.09 & 43.49 & 0.26 & 2.23 & 7.53 & & & & & & & & 6 & 6 & 127 \\
\hline DP1 & 7 & 1 & 0.16 & 43.56 & 0. & 2. & 7.61 & & 0 & & & & & & & & 19 \\
\hline 89 & 7 & 1 & 0.26 & 43.66 & 0.31 & 2 & 7.6 & 24 & 0 & & & & & & & & 4 \\
\hline 90 & 7 & 1 & 0.36 & 43.76 & 0. & & & 24 & & & & & & & & & 111 \\
\hline & 7 & 1 & 0.46 & 43.86 & 0.32 & 2.24 & 7.6 & 24.0 & 0. & & $=$ & 5 & 1. & 1 & 5 & & 117 \\
\hline DP1 4692 & 7 & 1 & 0.56 & 43.96 & 0.37 & 2.17 & 7.7 & 23.9 & 0.233 & 5 & 0.63 & 0. & 0.99 & .01 & 3 & 3 & 4 \\
\hline DP1 4693 & 7 & 1 & 0.66 & 44.06 & 0.42 & 2.1 & 7.7 & 23.9 & 0.268 & 8 & 0.7 & 0.6 & 0.9 & 9 & 3 & & 8 \\
\hline DP1 & 7 & 1 & 0.76 & 44.16 & 0.35 & 2. & 7.8 & 24.1 & & & & & 1. & & & & 116 \\
\hline DP & 7 & 1 & 0.86 & 44.26 & 0.33 & 2.2 & 7.8 & 230 & 0 & & & & & & & & 3 \\
\hline DP1 & 7 & 1 & 0.96 & 44.36 & 0. & 2 & 7. & & & & & & & & & & 27 \\
\hline & 7 & 1 & 1.06 & 44.46 & 0 . & & & & & & & & & & & & 1 \\
\hline 98 & 7 & 1 & 1.16 & 44.56 & 0.32 & 2. & 7.83 & 24.1 & 0 . & & & & 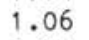 & & & & 6 \\
\hline DP1 1 & 7 & 1 & 1.26 & 44.66 & 0.28 & 2.32 & 7.8 & 24.1 & 0.287 & .00 & .7 & 0.6 & 1.13 & .06 & 4 & 66 & 117 \\
\hline DP1 4700 & 7 & 1 & 1.36 & 44.76 & 0.29 & 2.27 & 7.6 & 23.6 & 0.3 & 7 & 0.80 & & 1.09 & & & & 18 \\
\hline DP14701 & 7 & 1 & 1.46 & 44.86 & 0.32 & 2.21 & 7.7 & 23.8 & 0.2 & & 0.7 & & 1. & & & & 17 \\
\hline DP1 & 7 & 2 & 0.05 & & 0.32 & 2. & 7.7 & 23 & 0 & & 0 & & 1 & & & & \\
\hline 3 & 7 & 2 & 0.26 & & 0. & 2 & 7.7 & & 0 & & 0 & & & & 1 & & \\
\hline 04 & 7 & 2 & 0.36 & & 0. & & & & & & & & & & & & \\
\hline 705 & 7 & 2 & 0.56 & 46.46 & & & & & & & & & & & & & 15 \\
\hline 06 & 7 & 2 & 0.66 & 46.56 & 0 . & 2. & .6 & & & & & & & & & & 16 \\
\hline DP1 4707 & 7 & 2 & 0.76 & 46.66 & 0.38 & 2.13 & 7.72 & 23 & 0 & & $=$ & 2 & 1 & 6. & 3 & & 123 \\
\hline DP1 4708 & 7 & 2 & 0.86 & 46.76 & 0.38 & 2.18 & 7.85 & 23.3 & 0.386 & 2.81 & 0.97 & 0.7 & 1.11 & 6. & 0.073 & & 121 \\
\hline DP14709 & 7 & 2 & 0.96 & 46.86 & 0.35 & 2.2 & 7.66 & 23.5 & 0.348 & 6 & 0.91 & 3 & 1.16 & 6.41 & 2 & 63 & 119 \\
\hline DP1 & 7 & 2 & 1.08 & 46.98 & 0.41 & 2.07 & 7.8 & 23.2 & 0.383 & 2. & 0.88 & 0. & 3 & & & & 126 \\
\hline DP14711 & 7 & 2 & 1.16 & 47.06 & 0.33 & 2,1 & 7.6 & 22.9 & 0.4 & & 10 & & & & & & 128 \\
\hline 12 & 7 & 2 & 1.26 & 47. & & & & & & & & & & & & & 125 \\
\hline 13 & 7 & 2 & 1.36 & 47.26 & 0. & & & & & & & & & & & & 130 \\
\hline DP1 4714 & 7 & 2 & 1.46 & 47.36 & 0.31 & 2.30 & 7.3 & & 0 & & 1.24 & 0.6 & 1. & 6.03 & 32 & 44 & 121 \\
\hline DP1 4715 & 7 & 3 & 0.05 & 47.45 & 0.27 & 2.31 & $7 \cdot 3$ & 0 & 0.531 & 3 & 1.31 & 0. & 1.43 & 6.00 & .080 & & 125 \\
\hline DP1 4716 & 7 & 3 & 0.14 & 47.54 & 0.27 & 2.3 & 73 & 23.5 & & 3 & 1.1 & 0.6 & 1.34 & 6.10 & 0. & & 126 \\
\hline DP14717 & 7 & 3 & 0.26 & 47.66 & 0.28 & 2.24 & 7.32 & 23.1 & 0.5 & & 1.28 & 0.5 & & 5.93 & & & 119 \\
\hline 718 & 7 & 3 & 36 & 47.76 & 0.28 & 2.23 & 7.39 & 23.2 & 0.5 & 3. & 1.38 & & 1. & 5.93 & & & 118 \\
\hline & 7 & 3 & & & 0.31 & & 7.3 & & & & & & & & & & 19 \\
\hline & 7 & 3 & 0.56 & & & & & 3 & 0 . & & 1.43 & & & & & & 26 \\
\hline 21 & 7 & 3 & 0.66 & 6 & & & & & & & & & & & & & 26 \\
\hline & 7 & 3 & 0.76 & .16 & 0 & 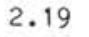 & & & & & 1.6 & & & & & & 128 \\
\hline DP1 4723 & 7 & 3 & 0.86 & 48.26 & 0.3 & 2,17 & 7. & 22.8 & 9 & & 1 & & & 2 & 6 & & 127 \\
\hline DP1 4724 & 7 & 3 & 0.96 & 48.36 & 0.3 & 21 & 7.16 & 2 & 0.645 & & 1.6 & & & & & & 124 \\
\hline DP1 4725 & 7 & 3 & 1.06 & 48.46 & 0.3 & 2.10 & 7.14 & 22.6 & 0.7 & & 1.77 & & & & & & 126 \\
\hline DP1 4726 & 7 & 3 & 1.16 & 8.56 & 0.35 & 2.11 & 7.37 & 23.1 & 0.662 & 3.08 & 1.66 & 0.5 & & & 0.077 & 0.089 & 125 \\
\hline & 7 & 3 & 26 & & $0.3+3$ & 2.12 & 7.42 & 22.9 & 0.695 & 3.03 & 1.73 & 0.5 & & & & & 123 \\
\hline & 7 & 3 & & & & & & & & & & & & & & & 123 \\
\hline & 7 & 3 & & & & & & & & & & & & & & & $c 1$ \\
\hline & 7 & 4 & 06 & 48.96 & & 2.12 & 7.48 & & & & 2.08 & & & 5.89 & & & 117 \\
\hline DP14731 & 7 & 4 & 0.16 & 49.06 & 0. & 2.0 & & 22. & & & & & & & 0.075 & & 115 \\
\hline DP1 4732 & 7 & 4 & 0.26 & 49.16 & 0.41 & 2.05 & 7.11 & 22.5 & 0.789 & & 2.03 & 0.474 & 1.26 & 5.53 & 0.075 & & 121 \\
\hline DP14733 & 7 & 4 & & & 0. & 2.11 & 7. & 22.8 & & & & 8 & & & 0.076 & & 125 \\
\hline DP1 4734 & 7 & 4 & & 49.36 & 0.37 & 2.11 & 7.14 & 22.8 & 0.8 & & 2.18 & 0.463 & 1.33 & 5.44 & 0.074 & & 127 \\
\hline 14735 & 7 & 4 & 0.56 & 49.46 & 0.31 & 2.10 & 7.10 & 22.8 & 0.840 & 3.25 & 2.18 & 0.445 & 1.35 & 5.34 & 0.072 & 0.097 & 128 \\
\hline
\end{tabular}


Table 2. (Continued).

\begin{tabular}{|c|c|c|c|c|c|c|c|c|c|c|c|c|c|c|c|c|c|}
\hline Lab ID & ڤั & 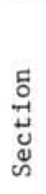 & 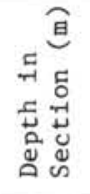 & 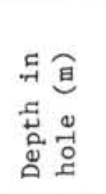 & $\mathrm{Na}$ & $\mathrm{Mg}$ & Al & Si & $\mathrm{P}$ & K & $\mathrm{Ca}$ & $\mathrm{T} i$ & Mn & $\mathrm{Fe}$ & $\mathrm{Ba}$ & S & $\mathrm{H}_{2} \mathrm{O}$ \\
\hline 4736 & 7 & 4 & 0.66 & & 35 & 2.14 & 96 & . & 886 & 25 & 5 & 3 & .40 & .42 & .078 & .105 & 25 \\
\hline DP1 4737 & 7 & 4 & 0.76 & 9.66 & 39 & & & & & & & & .35 & & & & \\
\hline DP1 4738 & 7 & 4 & 0.86 & 49.76 & 0.36 & 2.26 & 6.86 & 23.1 & 0.858 & & 2.25 & 495 & .37 & & & & 122 \\
\hline DP14739 & 7 & 4 & 0.96 & 49.86 & .34 & 2.18 & 7.05 & 22.8 & 0.845 & 7 & 2.16 & .415 & 1.51 & & 4 & & 130 \\
\hline 14740 & 7 & 4 & .06 & & 34 & & 7.07 & 22.9 & 0.832 & & 2.15 & .406 & 1.55 & .19 & .078 & & 125 \\
\hline DP1 4741 & 7 & 4 & 1.16 & .06 & 33 & 2.16 & 7.05 & 22.7 & & & 2.10 & 4 & & & & & 128 \\
\hline DP1 4742 & 7 & 4 & 1.26 & 50.16 & 40 & 2.16 & 7.10 & 22.9 & 0.779 & & 2.03 & & & & 0.077 & .081 & 129 \\
\hline DP1 4743 & 7 & 4 & 1.36 & & & 2.10 & & & & & & & & & 0.073 & & 128 \\
\hline DP1 4744 & 7 & 4 & 1.46 & 50.36 & 5 & 2.12 & 6.89 & 22.9 & 0.686 & & 1.85 & 0.472 & .33 & & & & 131 \\
\hline DP1 4745 & 7 & 5 & 0.06 & 50.46 & 0.31 & 2.11 & 7.12 & 22.7 & & & & 379 & & & 2 & & 131 \\
\hline DP1 4746 & 7 & 5 & 0.16 & 50.56 & 34 & 2.14 & 7.18 & 22.6 & 0. & & 2 & & & & 3 & & 125 \\
\hline DP1 4747 & 7 & 5 & 0.26 & 58.66 & 33 & 2.14 & 7.27 & 23.0 & 0. & 0 & 1.95 & 3 & .38 & 5 & & & 118 \\
\hline DP1 4748 & 7 & 5 & 0.36 & 50.76 & 3 & 2.15 & 7.26 & 22.9 & 0.8 & & 2.02 & & & & & & 116 \\
\hline DP14749 & 7 & 5 & 0.46 & & & 2 & 7. & & & & & & & & & & 119 \\
\hline DP1 & 7 & 5 & 0.56 & 50.96 & 0 & 2.13 & 7.30 & 23.0 & & & 2. & & & & .076 & 96 & 117 \\
\hline DP14751 & 7 & 5 & 0.66 & 51.06 & 4 & 2.14 & 7.3 & 23.2 & 0.7 & & & & 14 & & .077 & & 18 \\
\hline DP1 & 7 & 5 & 0.76 & 51.16 & 0. & 2.12 & 7.24 & 22.9 & 0 & & & & 1.46 & & 6 & 5 & 120 \\
\hline DP14753 & 7 & 5 & 0.86 & 51.26 & 0.34 & 2.10 & 7.12 & 22.5 & & & & & 1.39 & 5. & 5 & & 116 \\
\hline DP1 4754 & 7 & 5 & 0.96 & 51.36 & 0. & 2.0 & & & & & 2.56 & 0 . & 1.39 & 5. & 16 & & 116 \\
\hline DP1 & 7 & 5 & 1.06 & 51.46 & 6 & 2.06 & 7. & .7 & & & 2.48 & 0. & 1.38 & & & & 118 \\
\hline DP1 4756 & 7 & 5 & 1.16 & 51.56 & .38 & 2.06 & 7.14 & 22.7 & 0. & 9 & 2.50 & 0. & 1.46 & 4.97 & 4 & 22 & 119 \\
\hline DP1 4757 & 7 & 5 & 1.26 & 51.66 & 0.38 & 1.98 & 6.92 & 21.8 & 1.138 & 1 & 2.86 & 0 & 1.42 & 4.82 & 4 & & 116 \\
\hline DP1 4758 & 7 & 5 & 1.36 & 51.76 & & 2.07 & 7.2 & 22.9 & & & 2 & & 1.42 & & 7 & & 118 \\
\hline DP1 4759 & 7 & 5 & 1.46 & 51.86 & 8 & 2.07 & 7.15 & 4 & 1 & & 2 & & & & 7 & & 17 \\
\hline 760 & 7 & 6 & 0.06 & 51.96 & 0 & 2.09 & 7.10 & 4 & 0 & & 2 & 1 & 7 & & 4 & & 115 \\
\hline 761 & 7 & 6 & 0.16 & 52.06 & 0 & 2.16 & 7. & 22 & 6 & & 2 & & & & & & 1 \\
\hline 762 & 7 & 6 & 0.26 & 52.16 & & 2.17 & 7 & 23.0 & & & 8 & & & & & & 12 \\
\hline DP1 1 & 7 & 6 & 0.36 & 52 & 0 & 2. & 7 & 23 & & & & & & & & & 16 \\
\hline 64 & 7 & 6 & 0.46 & 36 & & & & & & & & & & & & & 112 \\
\hline 1765 & 7 & 6 & 0.56 & 2.46 & 4 & 2.15 & 7 & 2.9 & & & 2 & & 2 & 5.2 & 6 & & 114 \\
\hline 4766 & 7 & 6 & 0.66 & 2.56 & & 2.17 & 7 & 2.9 & & & & & & & & & 110 \\
\hline & 7 & 6 & 0.76 & .66 & & 1 & & & & & & & 8 & & 5 & & 111 \\
\hline & 7 & 6 & 0.86 & 52.76 & & 2.17 & & & & & 1.95 & & 31 & & 8 & & 112 \\
\hline DP1 4769 & 7 & 6 & 0.96 & 52.86 & & 2.19 & 7.23 & & 4 & & 1.8 & 0.3 & & & 6 & & 111 \\
\hline DP1 4770 & 7 & 6 & 1.06 & 52.96 & 0 & 2.22 & 7.39 & 23.4 & & & 10 & & & & 8 & & 112 \\
\hline DP14771 & 7 & 6 & 1.16 & 53.06 & 0.36 & 2,18 & 7.27 & 23.1 & & & & & & & 3 & & 10 \\
\hline DP1 & 7 & 6 & 1.26 & & & & & & & & & & & & 5 & & 109 \\
\hline$D P_{2}>$ & 7 & 6 & 36 & & 3 & 2.16 & & & & & 1. & & 1 & & & & 114 \\
\hline 4774 & 7 & 6 & 46 & & 5 & 2.1 & 7 & 4 & 8 & 3 & 1. & & 1.26 & & .077 & & 111 \\
\hline DP1 4775 & 7 & 7 & 06 & 46 & c & 2.1 & 7 & & & & & & 1.27 & & 0.074 & 71 & 109 \\
\hline DP1 4776 & 7 & 7 & 0.16 & & & & & & & & 0. & & 1.31 & & 73 & & 110 \\
\hline DP1 4777 & 7 & 7 & 0.26 & 53.66 & 0.36 & 2.17 & & & 0.3 & & 1. & & 1.35 & & 0.076 & & 09 \\
\hline DP1 4778 & 7 & 7 & 0.36 & 53.76 & 0.37 & 2.18 & 7.51 & 23.8 & & & & & & & & & 10 \\
\hline DP14779 & 7 & 7 & & & 0. & & & 24.2 & & & & & & & 8 & & 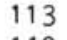 \\
\hline DP1 4780 & 8 & 1 & 26 & 6 & 0 & 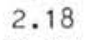 & 7. & 23 & & & 1 & & 12 & & 7 & & 119 \\
\hline 81 & 8 & 1 & 0.36 & & 0 & 2,1 & $73^{2}$ & 23.5 & 0 & & 1.2 & & 1.35 & 5. & 6 & & 120 \\
\hline & 8 & 1 & & & & 2 & & 3 & & & 1 & & & & & & 128 \\
\hline DP14783 & 8 & 1 & 0.56 & 54.66 & & 2.1 & & 23.3 & & & 1.60 & & & & 0.080 & & 123 \\
\hline DP1 4784 & 8 & 1 & 0.66 & 54.76 & 0. & 2.08 & 6.97 & 22.6 & & & 1.93 & & 1.81 & 4.99 & 0.077 & & 124 \\
\hline DP1 4785 & 8 & 1 & 0.76 & 54.86 & 0. & 2.09 & & 23.1 & & & & & & & & & \\
\hline DP1 4786 & 8 & 1 & 0.86 & 54.96 & & 2.22 & & 23.0 & & & & & & & & & 128 \\
\hline & 8 & 1 & 0.96 & & & 2.16 & & 22.5 & & & 1.86 & & 3 & & 90 & & 121 \\
\hline & 8 & 1 & & & & & & 22.8 & & & 1.41 & & & & & & 128 \\
\hline 4789 & 8 & 1 & 18 & & & 2.2 & & 22.4 & & & 2. & & & & & & 115 \\
\hline & 8 & 1 & 1 & 6 & & 2 & 6 & 22.7 & 0 & & 1.74 & & & 5. & 0.089 & & 123 \\
\hline & 8 & 1 & 1.3 & 55.46 & 0.2 & & & 9. & & & 23. & & & & & & 58 \\
\hline $0=14192$ & 8 & 1 & 1.46 & 55.56 & 0.3 & 2.22 & 6.78 & 22.7 & 0.721 & 3.42 & 2.37 & & & & 0.081 & & 116 \\
\hline DP1 4793 & 8 & 2 & 0.05 & 55.65 & 0.3 & 2.28 & 6.56 & 22.7 & & & 2.06 & & 1.62 & & 0.079 & & 118 \\
\hline DP1 4794 & 8 & 2 & 0.22 & & & 1.92 & & 19.5 & & & 6.71 & & 1.63 & & & & 109 \\
\hline DP14795 & 8 & 2 & 0.32 & 55.92 & & 2.32 & & 22.5 & & & 1.11 & & & 6.88 & 0.084 & & 133 \\
\hline DP1 4796 & 8 & 2 & .67 & & 0.2 & 2.17 & 6.67 & 22.6 & 0.446 & & 2.42 & & 1.58 & & & & 121 \\
\hline 797 & 8 & 2 & 0.78 & & & & & 21.2 & & & & & & & & & 122 \\
\hline 1) 14790 & 8 & 2 & & & 0.29 & 2.18 & & 23.0 & 0.419 & 3.37 & 1.64 & 0.371 & 1.54 & 5.62 & 0.078 & 0.075 & 123 \\
\hline
\end{tabular}


Table 2. (Continued).

\begin{tabular}{|c|c|c|c|c|c|c|c|c|c|c|c|c|c|c|c|c|c|}
\hline Lab ID & ๖్ّ & 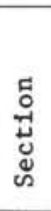 & 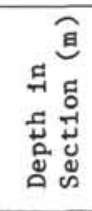 & 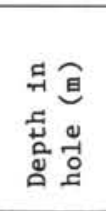 & $\mathrm{Na}$ & $\mathrm{Mg}$ & Al & Si & $P$ & $\mathrm{~K}$ & $\mathrm{Ca}$ & T1 & $\mathrm{Mn}$ & $\mathrm{Fe}$ & $\mathrm{Ba}$ & $\mathrm{S}$ & $\mathrm{H}_{2} \mathrm{O}$ \\
\hline D & 8 & 2 & 16 & & & & & & & & & & & & & 64 & 122 \\
\hline & 8 & 2 & 26 & & & & & & & & & & & & & & 118 \\
\hline DP1 & 8 & 2 & 1.36 & & 0.29 & 2.23 & & & & & & & & & & & 120 \\
\hline DP1 & 8 & 2 & 1.46 & 57. & 0.30 & 2.23 & & & 3 & 3 & & & & & & & 120 \\
\hline DP & 8 & 3 & 0.04 & 57. & 0.34 & 2.45 & & 23.0 & 0.491 & & & & & & 1 & & 130 \\
\hline DP1 & 8 & 3 & 0.66 & 57 & 32 & 2.40 & & 22.7 & 0.2 & & & & & & 0 & & 126 \\
\hline DP & 8 & 3 & 0.92 & 5 & 0.28 & 2.33 & 7 & 24.1 & 0.296 & 3 & & & & & & & 117 \\
\hline & 8 & 3 & 1.01 & 58 & 0.27 & 2.40 & 6.48 & 22.9 & .339 & 3 & 6 & 1 & 1.61 & 7. & 1 & & 133 \\
\hline DP1 4807 & 8 & 3 & 1.47 & 58 & 0.30 & 2.31 & 7.02 & 24.2 & 0 & 3 & 1 & 6 & 1.14 & 5.77 & 0.074 & 0.070 & 116 \\
\hline DP1 4808 & 8 & 4 & 0.03 & & 0.27 & 2.32 & 6 & 23.7 & 3 & & 1. & & 1. & & & & 112 \\
\hline DP14809 & 8 & 4 & 0.13 & & 0.31 & 2.35 & & 23.6 & & & 1.43 & & & & 0.073 & & 108 \\
\hline DP1 4810 & 8 & 4 & 0.23 & & 0.28 & 2.46 & & 23.0 & 0.430 & & 1.96 & & 1.41 & & & & 124 \\
\hline DP14811 & 8 & 5 & 0.74 & & 0.08 & 0.42 & & 2.1 & 0.031 & 0.26 & 35.69 & & 0.20 & & & 0.016 & 53 \\
\hline & 8 & 5 & .84 & & 0.10 & 0.42 & & 2.1 & 0.0 & 0.26 & 35.87 & & & & & & 54 \\
\hline DP & 8 & 5 & 0.95 & & 0.06 & 0.42 & & 2.1 & 0.0 & 0.27 & 35.65 & 0 & & & & & 53 \\
\hline DP1 & 8 & 6 & 0.77 & & 0.30 & 2.38 & 6. & 24.0 & 0.4 & 3.34 & & 2 & 2 & 5 & & 55 & 113 \\
\hline & 8 & 6 & & & 0.0 & 2.46 & & & & & & & & & & & 118 \\
\hline 816 & 8 & 7 & 0.39 & & 0.20 & 2. & & 24 & & & & & & & & & 121 \\
\hline DP1 4817 & 8 & C & 0.06 & 3.59 & 0.22 & 2.56 & 6.86 & 24.7 & 0.286 & 3.38 & 0.76 & 0.377 & 1.22 & 5.19 & 0.084 & 0.043 & 124 \\
\hline
\end{tabular}

Note: Depth in hole omitted for disturbed samples. 
Table 3. Elemental abundance (wt.\%) and water content (\% dry wt.) of Hole 576A samples.

\begin{tabular}{|c|c|c|c|c|c|c|c|c|c|c|c|c|c|c|c|c|c|}
\hline Lab ID & 㫕 & 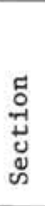 & 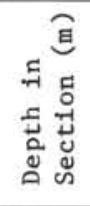 & 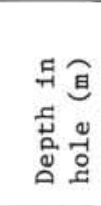 & $\mathrm{Na}$ & $\mathrm{Mg}$ & A1 & Si & P & K & $\mathrm{Ca}$ & $\mathrm{Ti}$ & $\mathrm{Mn}$ & $\mathrm{Fe}$ & $\mathrm{Ba}$ & $\mathrm{s}$ & $\mathrm{H}_{2} \mathrm{O}$ \\
\hline 732 & 1 & 1 & 0.24 & .44 & 0.32 & 1.75 & .10 & 26.8 & 0.053 & 2.49 & 0.31 & 0.394 & 0.18 & 4.56 & 0.086 & 0.046 & 149 \\
\hline DP19733 & 1 & 1 & 0.24 & .44 & 0.44 & 1.78 & .40 & 26.5 & 0.052 & 2.43 & .64 & 0.411 & 0.51 & 4.92 & .096 & 0.043 & 49 \\
\hline & 1 & 1 & 0.27 & 1.47 & 0.50 & 1. & 7.40 & 2 & 2 & 2.40 & 0.70 & 0.412 & & & 0. & 0.039 & \\
\hline 735 & 1 & 1 & 0.33 & 1.53 & 0.50 & 1.80 & 7.54 & 26.2 & 0.056 & 2.53 & 0.46 & 0.413 & 0.56 & 4.83 & 0.099 & & \\
\hline 736 & 1 & 1 & 0.45 & 1.65 & 0.58 & 1.80 & 7.53 & 26.2 & 0.054 & 2.59 & 0.44 & 0.417 & 0.55 & 4.82 & 0.095 & 0.037 & \\
\hline DP19737 & 1 & 1 & 0.49 & 1.69 & 0.53 & 1.83 & 7.53 & 26.2 & 0.054 & 2.61 & 0.44 & 0.421 & 0.45 & 4.82 & 0.094 & 0.046 & 120 \\
\hline DP19738 & 1 & 1 & 0.55 & 1.75 & 0.51 & 1.86 & 7.60 & 26.2 & 0.051 & 2.67 & 0.41 & 0.429 & 0.20 & 4.88 & 0.091 & 0.041 & \\
\hline DP19739 & 1 & 1 & 0.65 & 1.85 & 0.47 & 1.84 & 7.56 & 26.0 & 0.052 & 2.61 & 0.45 & 0.427 & 0.32 & 4.85 & 0.087 & 0.043 & \\
\hline DP19740 & 1 & 1 & 0.74 & 1.94 & 0.46 & 1.86 & 7.52 & 26.0 & 0.054 & 2.57 & 0.49 & 0.416 & 0.51 & 4.80 & 0.096 & 0.060 & 145 \\
\hline DP1S & 1 & 1 & 0.89 & 2.09 & 0.75 & 1.99 & 7.85 & 24.6 & 4 & 2.77 & & 1 & & 5.33 & & & 40 \\
\hline DP & 1 & 2 & 0.14 & 2.84 & 0.30 & 1.76 & 7. & 9 & & 2.54 & 24 & 0.393 & 0.09 & 4.54 & 8 & & 01 \\
\hline DP19743 & 1 & 2 & 0.20 & 2.90 & 0.38 & 1.74 & 7.31 & 27.1 & 0.050 & 2.52 & 0.33 & 0.403 & 0.11 & 4.67 & 0.098 & 0.014 & \\
\hline 44 & 1 & 2 & 0.30 & 3.00 & 0.30 & 1.68 & 7.03 & 26.8 & 0.049 & 2.42 & 0.27 & 0.380 & 0.11 & 4.42 & 0.097 & 0. & \\
\hline DP19745 & 1 & 2 & 0.39 & 3.09 & 0.26 & 1.65 & 7.11 & 27.1 & 0.053 & 2.38 & 0.27 & 0.378 & 0.22 & 4.43 & 9 & 0.014 & 232 \\
\hline DP1 & 1 & 2 & 0.48 & 3.18 & 0.31 & 1.65 & 6.96 & 26.5 & 0.052 & 2.42 & 0.34 & 0.379 & 0.26 & 4.46 & 0.091 & 0.032 & \\
\hline DP19747 & 1 & 2 & 0.58 & 3.28 & 0.45 & 1.75 & 7.28 & 26.2 & 0.054 & 2.54 & 0.42 & 0.398 & 0.34 & 4.64 & 0.088 & 1 & \\
\hline 48 & 1 & 2 & 0.64 & 3.34 & 0.53 & 1.77 & 7.38 & 26.3 & 0.055 & 2.59 & 0.43 & 0.412 & 0.42 & 4.73 & 0. & 37 & 138 \\
\hline 49 & 1 & 2 & 0.67 & 3.37 & 0.47 & 1.7 & 7.41 & 26.4 & 4 & 2.56 & 0.39 & 0.4 & & & & & \\
\hline 750 & 1 & 2 & 0.75 & 3.45 & 0.50 & 1.79 & 7. & 26.2 & 0 & 2.61 & 12 & 0. & 0 . & 4. & 7 & 7 & \\
\hline 751 & 1 & 2 & 0.85 & 3.55 & 0.42 & 1.82 & 7.54 & 26.4 & 0.054 & 2.61 & 0.41 & 0.410 & 0.35 & 4. & 0. & & \\
\hline 52 & 1 & 2 & 0.94 & 3.64 & 0.43 & 1.75 & 7.46 & 26.3 & 0.058 & 2.56 & 0.48 & 0.399 & 0.51 & 4.57 & 0. & 0. & 143 \\
\hline 753 & 1 & 2 & 1.09 & 3.79 & 0.48 & 1.82 & 7.51 & 26.1 & 0.057 & 2.58 & 0.48 & 0.403 & 0.39 & 4.72 & & 1 & 134 \\
\hline 754 & 1 & 2 & 1.34 & 4.04 & 0.43 & 1.91 & 7.58 & 26.2 & 0.054 & 2.67 & 0.42 & 0.418 & 0.27 & 4.86 & 0. & 0.056 & 134 \\
\hline DP19755 & 1 & 3 & 0.34 & 4.54 & 0.46 & 1.84 & 7.56 & 26.2 & 0.055 & 2.60 & 0.44 & 0.411 & 0.33 & & & & 134 \\
\hline DP & 1 & 3 & 0.84 & 5.04 & 0 & 1. & 7.60 & 0 & 0 & 2.66 & 0.37 & 9 & & 4. & 6 & 0 & 129 \\
\hline 57 & 1 & 3 & 1.03 & 5.23 & 0.41 & 1.8 & 7.52 & 26.0 & 0 & 2.57 & 7 & 0. & 2 & 4. & 7 & 0 . & \\
\hline 58 & 1 & 4 & 0.17 & 5.87 & 0.39 & 1.98 & 7.63 & 26.1 & 0.052 & 2.67 & 0.38 & 0.418 & 0.33 & 4.81 & 0.094 & 0.052 & \\
\hline 59 & 1 & 4 & .0 .23 & 5.93 & 0.51 & 1.95 & 7.70 & 26.0 & 0.053 & 2.66 & 0.34 & 0.421 & 0.33 & 4.87 & 0.090 & & \\
\hline & 1 & 4 & 0.35 & 6.05 & 0.51 & 1.87 & 7.52 & 26.3 & 0.054 & 2.55 & 0.36 & 0.4 & .44 & 4.65 & & 0 . & \\
\hline 761 & 1 & 4 & 0.44 & 6.14 & 0.44 & 1.91 & 7.58 & 26.2 & 0.054 & 2.59 & 0.39 & 0.411 & 0.41 & 4.75 & & & 143 \\
\hline DP1 & 1 & 4 & 0.74 & 6.44 & 0.39 & 1.91 & 7.57 & 26.1 & 0.054 & 2.58 & 0.36 & 0.416 & 0.41 & 4. & 0. & 29 & 155 \\
\hline & 1 & 5 & 0.05 & 7.25 & & 1.95 & 7.60 & 26.4 & 0.052 & 2.59 & & & & & & & \\
\hline DF & 1 & 5 & 0.15 & 5 & 0 & 1.96 & 7. & 1 & 2 & 1 & 6 & 9 & 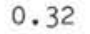 & 4. & 8 & 5 & \\
\hline 65 & 1 & 5 & 0.24 & 7.44 & 0. & 1.83 & 7.3 & 5 & 2 & 2. & 3 & 5 & 0.46 & 4. & 0. & 5 & 182 \\
\hline 66 & 1 & 5 & 0.49 & 7.69 & 0.44 & 1.90 & 7.44 & 26.2 & 0.050 & 2.57 & 0.44 & 0.408 & 0.43 & 4.70 & 0. & 0. & 135 \\
\hline 767 & 1 & 5 & 0.53 & 7.73 & 0.50 & 1.89 & 7.44 & 26.2 & 0.051 & 2.57 & 0.45 & 0.406 & .40 & 4.68 & 0.084 & 0.039 & \\
\hline 68 & 1 & 5 & 0.74 & 7.94 & 0.55 & 1.59 & 6.86 & 26.5 & 0.044 & 2.56 & 0.49 & 0.337 & .35 & 3.71 & 0. & 0 & 113 \\
\hline 169 & 1 & 5 & 0.99 & 8.19 & 0.44 & 1.97 & 7.54 & 26.0 & 0.049 & 2.63 & 0.36 & 0.409 & 0.35 & 4. & & & \\
\hline & 1 & 5 & 1.05 & & 0.61 & 1.94 & 7.56 & 26.2 & 0.047 & 2.64 & 0.35 & 0.413 & 0.24 & 4. & & & \\
\hline & 1 & 5 & 1.15 & & & 1.91 & 7.58 & & & & & & & & & & \\
\hline & 1 & 5 & 1 & 4 & 0 & 1. & 7. & 2 & 9 & 2. & 0. & 0. & 0. & 4. & & & 157 \\
\hline 564 & 1 & 5 & 1.27 & 8.47 & 0 & 1. & 7. & 3 & 48 & 2.67 & 0.30 & 0.416 & 0.25 & 4.8 & & & \\
\hline 9773 & 1 & 6 & 0.33 & 9.03 & 0.3 & 1.93 & 7.5 & 26.1 & 0 . & 2. & 0.34 & 0. & 0. & 4. & 0 . & 0. & \\
\hline 774 & 1 & 6 & 0.35 & 9.05 & 0.5 & 1.91 & 7.5 & 26.1 & 054 & 2.59 & 0.35 & 0. & 0.2 & 4. & 0. & & \\
\hline D. & 1 & 6 & 0.45 & 9.15 & .4 & 1.91 & 7.49 & 26.2 & 0.054 & 2.57 & 0.34 & 0.414 & 0.45 & 4. & 0.095 & & \\
\hline DP19776 & 1 & 6 & 0.54 & 9.24 & 0.3 & 1.85 & 7.27 & 26.2 & 0.0 & 2.49 & 0.30 & 0.399 & 0.51 & 3 & 9 & 0. & 192 \\
\hline DP19777 & 1 & 6 & 0.60 & 9.30 & 0.44 & 1.86 & 7.31 & 26.2 & 0.054 & 2.54 & 0.35 & 0.392 & 0.51 & 4. & 0. & & \\
\hline 778 & 1 & 6 & 0.70 & & 0.60 & 1.9 & 7.52 & 26.4 & 0.051 & 2.57 & & & & & & & \\
\hline & 1 & 6 & 0.79 & & 0.43 & 1.91 & 7.47 & 4 & & 2.61 & 0.38 & 0.410 & 0.46 & 4. & & & 47 \\
\hline & 1 & C & & 80 & 0 & 1. & $7 \cdot 3$ & & & 2.65 & 0.44 & 0. & 0.38 & 4. & 5 & & \\
\hline & 2 & 1 & 0.55 & & 0 & 1.9 & 7.55 & 26.5 & 0.055 & 2.58 & 0.32 & 0.405 & 0.37 & 4.56 & 0.102 & 0. & \\
\hline & 2 & 1 & 0.65 & & 0.5 & 1.93 & 7.54 & 26.6 & .054 & 2.57 & 0.30 & 0.399 & 0.39 & 4. & 0. & & \\
\hline & 2 & 1 & 0.74 & & 0.40 & 1.9 & 7.53 & 26.5 & 56 & 2. & 0.31 & 0.397 & 0.45 & 4. & 0.0 & 0 . & 179 \\
\hline 783 & 2 & 1 & 0.78 & 08 & 0.39 & 2.00 & 7.53 & 26.3 & 0.059 & 2.50 & 0.32 & 0.399 & 0.45 & 4.61 & 0.0 & & \\
\hline DP19784 & 2 & 1 & 0.85 & & 0.57 & 1.9 & 7.46 & 25.4 & 0.058 & 2.47 & 0.26 & 0.386 & 0.38 & 4.61 & & & \\
\hline & 2 & 1 & 0.95 & & & 2.00 & 7.51 & 25.9 & & & 0.34 & & & & & & \\
\hline & 2 & 1 & 0.99 & 29 & 0.42 & 1.99 & 7.51 & 26.0 & 0.0 & 2.58 & 0.37 & 0.411 & 0.40 & 4. & 0.098 & 0.041 & 143 \\
\hline & 2 & 1 & 1.24 & .54 & 0.32 & 1.98 & 7.54 & 26.0 & 0.054 & 2.48 & 0.35 & 0.408 & 0.47 & 4. & 0.095 & 0.033 & 171 \\
\hline & 2 & 1 & 1.30 & & 56 & 2.00 & 7.60 & 26,1 & .054 & 2.48 & 0.30 & 0.406 & 0.44 & 9 & 0.097 & 0.029 & \\
\hline & 2 & 1 & 1.39 & .69 & 0.41 & 1.96 & 7.46 & 26.2 & 0.056 & 2.41 & 0.34 & 0.398 & 0.49 & 4.61 & 0.099 & 0.0 & 184 \\
\hline & 2 & 2 & 0.05 & & & 1.98 & & 26.1 & 0.0 & 2.48 & 0.37 & 0.409 & 0.43 & 4.72 & 0.095 & 0.011 & \\
\hline DP1 & 2 & 2 & 0.58 & & 0.67 & 1.9 & 7.65 & 26.2 & 0.062 & 2.42 & 0.28 & 0.402 & 0.49 & 4.71 & 0.091 & 0.0 & \\
\hline DP19791 & 2 & 2 & 0.69 & 12.49 & 0.36 & 2.01 & 7.58 & 26.1 & 0.059 & 2.50 & 0.34 & 0.410 & 0.48 & 4.69 & 0.092 & 0.028 & 10 \\
\hline
\end{tabular}




\begin{tabular}{|c|c|c|c|c|c|c|c|c|c|c|c|c|c|c|c|c|c|}
\hline Lab ID & 㫕 & 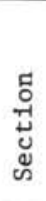 & 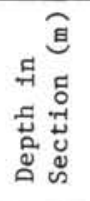 & 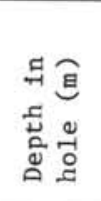 & $\mathrm{Na}$ & $\mathrm{Mg}$ & Al & Si & P & K & $\mathrm{Ca}$ & $\mathrm{T} 1$ & Mn & $\mathrm{Fe}$ & $\mathrm{Ba}$ & $\mathrm{S}$ & $\mathrm{H}_{2} \mathrm{O}$ \\
\hline 19792 & 2 & 2 & .73 & .53 & .34 & 1.97 & 53 & 9 & 059 & 2.50 & 0.33 & 0.406 & 0.44 & 66 & 090 & .023 & \\
\hline 19793 & 2 & 2 & .89 & .69 & .50 & 1 & 7.60 & 26.1 & .058 & .40 & 0.31 & 0.398 & 0.52 & 65 & & & 183 \\
\hline DP19794 & 2 & 2 & 1.24 & 13.04 & 0.44 & 1.99 & 7.57 & 26.1 & .048 & 2.63 & 0.32 & 0.415 & 0.36 & 4.73 & .090 & .032 & 144 \\
\hline DP18566 & 2 & 2 & 1.42 & 13.22 & 0.31 & 2.05 & 7.57 & 26.1 & 0.047 & 2.57 & 0.29 & 0.421 & 0.42 & 4.76 & 0.093 & 0.025 & \\
\hline DP19795 & 2 & 3 & 0.28 & 13.58 & 0.41 & 2.02 & 7.55 & 25.8 & 0.048 & 2.70 & 0.34 & 0.421 & 0.40 & 4.84 & 0.097 & 0.046 & \\
\hline DP19796 & 2 & 3 & 0.36 & 13.66 & 0.52 & 2.00 & 7.66 & 26.0 & 0.048 & 2.74 & 0.31 & 0.420 & 0.33 & 4.84 & & 38 & \\
\hline DP19797 & 2 & 3 & 0.46 & 13.76 & 0.48 & 1.99 & 7.58 & 25.9 & 0.053 & 2.55 & 0.25 & 0.404 & 0.39 & 4.65 & 0.096 & 18 & \\
\hline DP19798 & 2 & 3 & 0.49 & 13.79 & 0.50 & & 7.61 & & & & & & & & & & 166 \\
\hline DP18567 & 2 & 3 & 0.67 & 13.97 & 0.34 & 2.06 & 7.60 & 26 & 4 & 2. & 0 & 0 & 0 & & 93 & 7 & \\
\hline DP19799 & 2 & 4 & 0.03 & 14.83 & 0.50 & 2.15 & 7.59 & 26 & 4 & & 0 & 3 & 0 & 4.49 & 1 & 34 & \\
\hline DP19800 & 2 & 4 & 0.10 & 14.90 & 0.50 & 2.32 & 7.48 & 26.0 & 0.052 & 2.11 & 0.31 & 0.336 & 0.43 & 3.97 & 0.075 & 0. & \\
\hline DP19801 & 2 & 4 & 0.15 & 14.95 & 0.62 & 2.36 & 7.41 & 26.6 & 0.051 & 1.91 & 0.35 & 0.284 & 0.32 & 3.38 & .069 & & \\
\hline DP19802 & 2 & 4 & 0.20 & 15.00 & 0.53 & 2.19 & 7.55 & 26.3 & 0.059 & 2.28 & 0.33 & 0.376 & 0.49 & 4.33 & 0.085 & 0.032 & \\
\hline DP19803 & 2 & 4 & 0.29 & 15.09 & 0.75 & 1.97 & 7.70 & & 0.065 & & & & 0.50 & & & & 162 \\
\hline DP19804 & 2 & 5 & 0.10 & 16.40 & 0.59 & 2.07 & 7.73 & 26 & & 2. & & & & & & & \\
\hline DP19805 & 2 & 5 & 0.20 & 16.50 & 0.68 & 2.00 & 7. & 25 & 8 & 2. & & 9 & 0 & 3 & 4 & 0.031 & \\
\hline DP19806 & 2 & 5 & 0.29 & 16.59 & 0.43 & 1.96 & 7.57 & 25.8 & 0.062 & 2.48 & 0.40 & 0.417 & 0.53 & 4.79 & 0.094 & 0.034 & 157 \\
\hline DP18568 & 2 & 5 & 0.41 & 16.71 & 0.27 & 2.04 & 7.65 & 25.4 & 0.057 & 2.38 & 0.47 & 0.410 & 0.56 & 4.89 & 0.088 & 0.031 & \\
\hline DP19807 & 2 & 6 & 1.19 & 18.99 & 0.69 & 1.87 & 7.93 & 25.5 & 0.062 & 2.52 & 0.42 & 0.427 & 0.63 & 5.10 & 0. & 0.022 & 136 \\
\hline DP19808 & 2 & 6 & 1.23 & 19.03 & 0.53 & 1.89 & 7.87 & 25.4 & 0.057 & 2.51 & 0.45 & 0.420 & 0.61 & & & & \\
\hline DP & 2 & 6 & 1.49 & 19.29 & 0.46 & 1.93 & 7.82 & 25.5 & 0.062 & 2.59 & 0.39 & 0.419 & 0.65 & 4.98 & & 0.039 & 134 \\
\hline DP19810 & 2 & 7 & 0.03 & 19.33 & 0.52 & 1.90 & 7.77 & 25.3 & 0.058 & 2.58 & 0.37 & 0.421 & 0.62 & 4.93 & & & \\
\hline DP1 & 2 & $C$ & & 19.50 & 0.43 & 1.98 & 7.90 & 25.5 & 0.055 & 2.62 & 0.41 & 9 & & & & & \\
\hline DP19811 & 3 & 1 & 0.05 & 23.05 & 0.59 & 1.92 & 7.8 & 25.3 & 0.060 & 2.67 & & 4 & & & & & \\
\hline DP19812 & 3 & 1 & 0.15 & 23.15 & 0. & 1. & 7. & 2 & 2 & 2.65 & & 7 & & & 9 & 2 & \\
\hline DP19813 & 3 & 1 & 0.24 & 23.24 & 0.51 & 1.91 & 7. & 25 & 4 & 2. & 0 & 0 & 9 & 3 & 81 & 0.040 & 124 \\
\hline DP19814 & 3 & 1 & 0.49 & 23.49 & 0.49 & 1.90 & 7.94 & 25.5 & 0.069 & 2.66 & 0.33 & 0.429 & 0.65 & 4.92 & 0.080 & 0.034 & 131 \\
\hline 15 & 3 & 1 & 0.74 & 23.74 & 0.51 & 1.91 & 7.85 & 25.2 & 0.064 & 2.64 & 0.34 & 0.431 & 0.65 & 4.95 & 0. & 0.0 & 138 \\
\hline 16 & 3 & 1 & 0.78 & 23.78 & 0 & 1. & 7.9 & 25.2 & 0.069 & 2.59 & 0.30 & 0.422 & 0.73 & 5 & 0. & 28 & \\
\hline DP19817 & 3 & 1 & 0.83 & 23.83 & 0.68 & 1.90 & 7.94 & 25.4 & 0.064 & 2.62 & 0.32 & 0.437 & 0.68 & & 88 & 27 & \\
\hline DP19818 & 3 & 1 & 0.93 & 23.93 & 0.68 & 1. & 7.91 & 2 & 0. & 2.54 & & 0. & & & & & \\
\hline DP19819 & 3 & 1 & 0.99 & 23.99 & 0.75 & 1. & 7.94 & 25.3 & 0.061 & 2.55 & & 0. & 3 & 0 & 7 & 9 & 124 \\
\hline DP19820 & 3 & 1 & 1.10 & 24.10 & 0.80 & 1.86 & 7.96 & 25.4 & 0. & 2.63 & 0 & 0.430 & 0.68 & 5.03 & 0.077 & 0.022 & \\
\hline DP19821 & 3 & 1 & 1.20 & 24.20 & 0.69 & 1.89 & 7.95 & 25.3 & 0.070 & 2.60 & 0.30 & 0.430 & 0.71 & 4.99 & 0.080 & 0.020 & \\
\hline DP19 & 3 & 1 & 1.24 & 24.24 & 0.63 & 1.90 & 7.8 & 25.3 & 0.073 & 2.61 & 0.34 & 0.440 & 0.69 & 5.01 & 0. & .033 & 126 \\
\hline DP19 & 3 & 2 & 0.91 & 25.41 & 0.55 & 1.94 & 7.8 & 25.3 & 0.0 & 2.78 & 0.33 & 0.435 & 0 . & 4.99 & 0.077 & & \\
\hline DP1 & 3 & 2 & 0.97 & 25.47 & 0.70 & 1.93 & 7.81 & 25.3 & 0.066 & 2.76 & 0.29 & 0.437 & 0.59 & 4.99 & 0.077 & 0. & \\
\hline DP19825 & 3 & 2 & 1.04 & 25.54 & 0.56 & 1.95 & 7.82 & 25.3 & 0.068 & 2.80 & 0.33 & 0.436 & 4 & & 77 & & \\
\hline DP1 & 3 & 3 & 0.94 & 26.94 & 0.51 & 2. & 7.15 & 24.5 & 0 . & 2.72 & & 0.512 & 0.98 & 51 & 11 & 66 & 122 \\
\hline DP19827 & 3 & 3 & 1.04 & 27.04 & 0.52 & 2. & 7. & 6 & 38 & 2.72 & & 0. & 0 & 53 & 16 & 57 & \\
\hline DP19828 & 3 & 3 & 1.14 & 27.14 & 0.60 & 2.14 & 7.26 & 24.8 & 97 & 2.72 & 3 & 0.516 & 0.99 & 5.57 & 0.113 & 0.047 & \\
\hline DP19829 & 3 & 3 & 1.19 & 27.19 & 0.51 & 2.11 & 7.20 & 24.6 & 0.190 & 2.72 & 0.66 & 0.514 & 0.98 & 5.53 & 0.114 & 0.060 & 120 \\
\hline DP19830 & 3 & 3 & 1.23 & 27.23 & 0.48 & 2.15 & 7.2 & 24.6 & 0.188 & 2.74 & 0.66 & 0.515 & 0.99 & 5.55 & 0.112 & 0. & \\
\hline DP19831 & 3 & 3 & 1.39 & 27.39 & 0.57 & 2.14 & 7.2 & 24.7 & 0.198 & 2.72 & 0.66 & 0.521 & 1. & 5.57 & 0.114 & 0.053 & 117 \\
\hline & 3 & 4 & 0.04 & & 0.58 & 2.09 & 7.2 & 24.7 & 0.229 & 2.71 & 0.73 & 0.523 & 1. & & 15 & & \\
\hline DP19833 & 3 & 4 & 0.12 & & 0.60 & 1.93 & & 25.2 & 0.063 & 2.94 & 3 & 33 & 19 & & & & \\
\hline DP19834 & 3 & 4 & 0.14 & & 0.61 & 1.92 & 7.86 & 25.2 & 65 & 2.94 & 0 & 0.434 & 0.50 & 7 & 74 & 0. & 96 \\
\hline DP18569 & 3 & 4 & 0.26 & & 0.50 & 1.98 & 7.92 & 25.1 & 0.065 & 2.88 & 0.34 & 0.431 & 0.65 & 10 & .077 & 10 & \\
\hline DP19835 & 3 & 4 & 1.08 & & 0.52 & 1.99 & 7.98 & 25.1 & 0.068 & 2.75 & 0.34 & 0.441 & 0.82 & 5.12 & 0.076 & 0.044 & \\
\hline DP19836 & 3 & 4 & 1.19 & & 0.59 & 1.93 & 8.02 & 25.0 & 0.073 & 2.72 & 0.33 & 0.444 & 0.77 & 5.18 & 0.079 & 0.033 & 108 \\
\hline P19837 & 3 & 5 & 0.05 & & 0.68 & 2.00 & 7.9 & 24.9 & 0.085 & 2.67 & 0.38 & 0.448 & 0.91 & $5 \cdot 30$ & 0.078 & 0.031 & \\
\hline P19838 & 3 & 5 & 0.11 & & 0.54 & 2.02 & 7.84 & 24.8 & 0.086 & 2.65 & 0.44 & 0.446 & 0.88 & 9 & 0.079 & 0.053 & 113 \\
\hline 39 & 3 & 5 & 1.24 & & 0.56 & 1.98 & 7.6 & 24.4 & 1 & 2.66 & 0.3 & 0.2 & 3 & & 74 & & \\
\hline DP19840 & 3 & 5 & 1.29 & & 0.58 & 2.01 & 7.78 & 24.8 & 0.098 & 2.68 & 0.40 & 0.438 & 0.95 & 22 & 0.076 & & 113 \\
\hline DP19841 & 3 & 6 & 0.04 & & 0.40 & 1.84 & 7.42 & 26.0 & 0.053 & 2.52 & 0.44 & 0.404 & 0.64 & 4.72 & 0.097 & 0.047 & \\
\hline DP19842 & 3 & 6 & 0.22 & & 0.76 & 2.01 & 7.82 & 24.4 & 0.120 & 2.72 & 0.46 & 0.463 & 1.12 & 5.36 & 0.072 & 0.033 & \\
\hline DP19843 & 3 & 6 & 0.29 & & 0.58 & 1.97 & 7.75 & 24.3 & 0.129 & 2.72 & 0.52 & 0.461 & 1.13 & 5.32 & 0.071 & 0.050 & 105 \\
\hline DP19844 & 3 & 6 & 0.88 & & 0.65 & 1.95 & 7.85 & 24.4 & 0.139 & 2.74 & 0.53 & 0.454 & 1.24 & 5.35 & 0.073 & 0.042 & \\
\hline DP19845 & 3 & 6 & 1.09 & 29.69 & 0.65 & 2.00 & 7.82 & 24.8 & 0.096 & 2.73 & 0.39 & 0.453 & 0.93 & 5.32 & 0.082 & 0.028 & 106 \\
\hline DP19846 & 3 & 6 & 1.19 & 29.79 & 0.62 & 2.00 & 7.79 & 24.6 & 0.105 & 2.71 & 0.41 & 0.449 & 0.99 & 5.26 & 0.077 & 0.033 & \\
\hline DP19847 & 3 & 6 & 1.29 & 29.89 & 0.60 & 2.00 & 7.83 & 24.8 & 0.109 & 2.73 & 0.42 & 0.453 & 0.96 & $5 \cdot 32$ & 0.082 & 0.033 & \\
\hline & 3 & 6 & 1.34 & 29.94 & 0.59 & 2.00 & 7.79 & 24.6 & 0.111 & 2.79 & 0.45 & 0.453 & 1.03 & 5.30 & 0.077 & 0.045 & 110 \\
\hline DP19849 & 3 & 6 & 1.49 & 30.09 & 0.49 & 2.01 & 7.81 & 24.6 & 0.112 & 2.79 & 0.49 & 0.459 & 1.10 & 5.29 & 0.074 & 0.044 & 114 \\
\hline
\end{tabular}


Table 3. (Continued).

\begin{tabular}{|c|c|c|c|c|c|c|c|c|c|c|c|c|c|c|c|c|c|}
\hline Lab ID & ڤ్ల & ஸू & 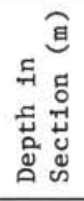 & 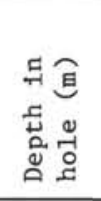 & $\mathrm{Na}$ & $\mathrm{Mg}$ & Al & $\mathrm{Si}$ & $\mathrm{P}$ & $\mathrm{K}$ & $\mathrm{Ca}$ & Ti & Mn & $\mathrm{Fe}$ & $\mathrm{Ba}$ & $\mathrm{S}$ & $\mathrm{H}_{2} \mathrm{O}$ \\
\hline DP10804 & 3 & $\mathrm{C}$ & & .50 & 0.89 & 1.86 & 7.84 & 24.2 & 0.120 & 2.80 & 0.56 & .455 & 1.28 & 5.31 & 0.074 & 0.020 & \\
\hline DP19850 & 4 & 1 & 0.24 & & 0.58 & 2.01 & 7.87 & 24.8 & .097 & .69 & 0.39 & .447 & .00 & .27 & .075 & 0.033 & 127 \\
\hline DP19851 & 4 & 1 & 0.45 & 29.35 & 0.67 & 1.92 & 7.84 & 24.0 & 0.132 & 2.70 & 0.49 & 0.445 & 1.27 & 5.26 & 0.072 & 0.039 & \\
\hline DP19852 & 4 & 1 & 0.49 & 29.39 & 0.67 & 1.91 & 7.89 & 24.1 & 0.132 & 2.71 & 0.48 & 0.440 & 1.24 & 5.23 & 0.072 & 0.042 & 124 \\
\hline DP19853 & 4 & 1 & 0.65 & 29.55 & 1.01 & 1.86 & 8.05 & 24.3 & 0.129 & 2.76 & 0.43 & 0.444 & 1.30 & 5.30 & 0.075 & 0.026 & \\
\hline DP19854 & 4 & 1 & 0.74 & 29.64 & 0.67 & 1.90 & 7.95 & 24.2 & 0.137 & 2.74 & 0.50 & 0.445 & 1.30 & 5.27 & 0.073 & 0.037 & 127 \\
\hline DP19855 & 4 & 1 & 0.78 & 29.68 & 0.71 & 1.88 & 7.94 & 24.0 & 0.133 & 2.72 & 0.49 & 0.447 & 1.33 & 5.29 & 0.072 & 0.046 & \\
\hline DP1 & 4 & 1 & 0.99 & 29.89 & 0.80 & 1.89 & 7.98 & 24.1 & 0.136 & 2.73 & 0.50 & 0.447 & 1.41 & 5.36 & 0.071 & 0.041 & 121 \\
\hline DP1 & 4 & 1 & 1.39 & & 0.65 & 1.94 & & 24.4 & 0.123 & 2.72 & 0.43 & 0.454 & .32 & & 71 & & 129 \\
\hline DF & 4 & 2 & 0.19 & 30 & 0.69 & 2. & 7. & 23.9 & & 2.79 & & & .39 & & 73 & 5 & \\
\hline DP & 4 & 2 & 1.06 & 6 & 0.74 & 1. & 8. & 2 & 8 & 2 & 0 & & 9 & & & & \\
\hline 859 & 4 & 2 & 1.14 & 3 & 0. & 1. & 8 & 2 & 0 & 8 & & 8 & 3 & & 1 & 37 & \\
\hline DP19860 & 4 & 2 & 1.22 & 31.62 & 0.74 & 1.96 & 7.93 & 23.7 & 0.166 & 2.58 & 0.52 & 0.441 & .53 & 5.38 & 0.071 & 0.045 & 128 \\
\hline 361 & 4 & 3 & 0.52 & 32.42 & 0.72 & 1.93 & 7.86 & 23.6 & 0.199 & 2.57 & 0.67 & 0.433 & 1.71 & 5.34 & 0.071 & 0.051 & 138 \\
\hline 362 & 4 & 3 & 0.76 & 32.66 & 0.69 & 1.97 & 7.79 & 23.5 & 0.197 & 2.57 & 0.67 & 0.434 & 1.67 & 5.33 & 0.073 & 0.048 & \\
\hline DP1 & 4 & 3 & 0.81 & 32.71 & 0.87 & 1.93 & 7.87 & 23.8 & 0.195 & 2.59 & 0.64 & 0.435 & 1.70 & 5.38 & 0.073 & 1 & \\
\hline DP19 & 4 & 3 & 0.93 & 32.83 & 1.15 & 1.91 & 7.80 & 23.8 & 0 & 2.56 & 0.63 & & & & & & \\
\hline DF & 4 & 3 & 0.97 & 3 & 0 . & 1. & 7. & 23 & 5 & 2. & 1 & 0 & 58 & & 1 & & 132 \\
\hline 866 & 4 & 3 & 1.12 & 33 & 0 . & 1. & 7 & 23 & 0 & 2. & 0 & 2 & 8 & & 1 & & 134 \\
\hline 501 & 4 & 4 & 0.11 & 33.51 & 0.81 & 1.92 & 7.62 & 22.9 & 0.205 & 2.52 & 0.68 & 0.443 & 1.85 & 5.48 & 0.073 & 0.051 & \\
\hline DP19868 & 4 & 4 & 0.26 & 33.66 & 0.73 & 1.96 & 7.74 & 23.3 & 0.223 & 2.54 & 0.70 & 0.446 & 1.84 & 5.60 & 0.070 & 0.066 & 143 \\
\hline DP1 & 4 & 4 & 0.67 & 34.07 & 0.77 & 1.99 & 7.48 & 23.0 & 0.362 & 2.58 & 1.05 & 0.463 & 2.24 & 5.50 & 0.077 & 0. & \\
\hline D & 4 & 4 & 0.76 & & 0.65 & 1.95 & 7.85 & 23.9 & 0.164 & 2.76 & 0.54 & 0.439 & .48 & & & & 120 \\
\hline DF & 4 & 4 & 0.87 & & 0 . & 1. & 7. & 24 & 8 & 2. & 0 & 3 & 47 & 5 & 3 & 5 & \\
\hline DP & 4 & 4 & 0.99 & & 0.93 & & 7. & 23 & & & & & 6 & & & & \\
\hline DP19873 & 4 & 4 & 1. & & 0. & 1. & 7. & 2. & 8 & 1 & 3 & 1 & 3 & 5. & 0. & 0. & 95 \\
\hline DP19874 & 4 & 4 & 1.35 & 34.75 & 1.01 & 1.92 & 7.68 & 23.1 & 0.347 & 2.51 & 0.90 & 0.453 & .09 & 5.42 & 0.0 & 0.0 & \\
\hline DP19875 & 4 & 5 & 0.07 & 34.97 & 0.84 & 1.95 & 7.90 & 23.8 & 0.187 & 2.65 & 0.59 & 0.454 & 1.79 & 5.36 & 0.073 & 0 . & \\
\hline 376 & 4 & 5 & 0.26 & 35.16 & 0.60 & 2.04 & 7.37 & 22.8 & 0.388 & 2.57 & 1.12 & 0.450 & 2.16 & 5.49 & 0.075 & 0.0 & \\
\hline DP & 4 & 5 & 0.37 & 35.27 & 0.69 & 1.99 & 7.31 & 22.6 & 0.518 & 2.58 & 1.40 & 0.440 & 2.09 & 5.42 & 0.076 & 0. & \\
\hline & 4 & 5 & 0.47 & 37 & 0.61 & 1.98 & 7.2 & 22.4 & 0.5 & 2.57 & 1.52 & 0.4 & .04 & 5. & 0. & & 134 \\
\hline & 4 & 5 & 0.69 & & 0.44 & 2. & 7. & 22.6 & 0 & 2.66 & 1 & 0 . & 3 & & & & 22 \\
\hline DP18571 & 4 & 5 & 0.73 & & 0 . & 2. & & 8 & & 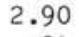 & 41 & 2 & 66 & 5 & 4 & & \\
\hline DP19881 & 4 & 6 & 0.21 & & 0.58 & 1. & & & & 2 & 0 & 32 & 6 & 4.88 & 0.071 & 0.059 & \\
\hline DP19882 & 4 & 6 & 0.34 & 36.74 & 0.55 & 2.01 & 7.90 & 23.4 & 0.318 & 2.84 & 0.82 & 0.388 & 1.77 & 4.95 & 0.072 & 0.071 & 116 \\
\hline DP19883 & 4 & 6 & 0.63 & 37.03 & 0.43 & 2.03 & 7.82 & 23.4 & 0.339 & 2.88 & 0.90 & 0.413 & 1.70 & 5.04 & 0.074 & 0.064 & \\
\hline DP19884 & 4 & 6 & 0.77 & 37.17 & 0.46 & 2.08 & 7.86 & 23.5 & 0.318 & 2.91 & 0.85 & 0.439 & 1.64 & 5.13 & 0.071 & 0.068 & 111 \\
\hline DP19 & 4 & 6 & 1.03 & 37.43 & 0.52 & 2.05 & 7.92 & 23.4 & 0.322 & 2.73 & 0.84 & 0.502 & 1.56 & 5.28 & 0.073 & 0.067 & 110 \\
\hline DP & 4 & 6 & & 56 & 0.47 & 2.09 & 7.95 & 23.4 & 0.270 & 2.82 & 0.73 & 0.483 & 1.56 & 5.34 & 0.071 & 0.055 & \\
\hline & 4 & C & & 75 & 1.21 & 2.02 & 8. & 23.9 & 0. & 2.87 & 9 & 0. & 1.69 & 5 & 0 & & \\
\hline & 5 & 1 & 0.09 & & & 2. & 8 & 2 & 0 & 3 & 31 & 0 & 59 & & & & \\
\hline 888 & 5 & 1 & 0.24 & & 0.37 & 2.04 & & & & & 33 & & 71 & 8 & 2 & 68 & 128 \\
\hline DP19889 & 5 & 1 & 0.75 & & 0.46 & 2.13 & 7.85 & 23.2 & 0 & 2. & 0.71 & 0.477 & 1.53 & 5. & 0 . & 0.058 & \\
\hline DP19890 & 5 & 1 & 0.89 & 99 & 0.39 & 2.07 & 7.92 & 23.3 & 0.278 & 2.80 & 0.73 & 0.479 & 1.53 & 5.29 & 0.073 & 0. & \\
\hline DP19 & 5 & 1 & 0.95 & 05 & 0.47 & 2.09 & 7.97 & 23.5 & 0.285 & 2.80 & 0.74 & 0.488 & 1.54 & 5.31 & 0.072 & 0. & \\
\hline DP19892 & 5 & 1 & 1.14 & 24 & 0.42 & 2.05 & 7.89 & 23.3 & 0.335 & 2.69 & 0.87 & 0.505 & 1.55 & 5.29 & 0.072 & 0. & 120 \\
\hline DP19 & 5 & 1 & 1.39 & & 0.43 & 2.03 & 7.93 & 23.4 & 0.346 & 2.65 & 0.89 & 0.5 & .58 & 5.36 & 0.072 & 0. & 120 \\
\hline & 5 & 2 & 0.24 & 84 & 0.44 & 1.98 & 7.85 & .1 & 0.360 & 2.59 & 0.93 & 0 & 0 & & 2 & & 120 \\
\hline & 5 & 2 & 0.43 & 3 & 0.48 & 1.96 & & 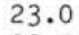 & $=$ & 2. & 9 & & 7 & & & & \\
\hline DP19896 & 5 & 2 & 0.50 & 41.10 & 0.62 & 1.98 & 7 & .5 & 50 & 2.65 & 0.90 & 0. & 58 & 5 & 0. & 0. & \\
\hline DP19897 & 5 & 2 & 0.64 & 41.24 & 0.55 & 1.95 & 7.88 & 23.2 & 0.354 & 2.59 & 0.91 & 0.542 & 1.57 & 5.48 & 0.072 & 0. & 122 \\
\hline DP19898 & 5 & 2 & 1.04 & 41.64 & 0.47 & 1.95 & 7.85 & $23^{\circ} .3$ & 0.365 & 2.64 & 0.95 & 0.551 & 1.50 & 5.52 & 0.070 & 0. & 119 \\
\hline DP19899 & 5 & 2 & 1.30 & 41.90 & 0.62 & 1.93 & 7.89 & 23.3 & 0.335 & 2.6 & 0.87 & 0.541 & .48 & 5.52 & 0.071 & 61 & \\
\hline DP & 5 & 2 & 1.49 & 42.09 & 0.48 & 1.94 & 7.83 & 23.2 & 0.356 & 2.64 & 0.92 & 0.543 & 1.48 & 5.48 & 0.071 & 0. & 120 \\
\hline DP19901 & 5 & 3 & 0.88 & 42.98 & 0.45 & 2.08 & 7.63 & 23.7 & 0.257 & 3.19 & 0.71 & 8 & 19 & & & & \\
\hline & 5 & 5 & 0.03 & 45.13 & 0.58 & 2.09 & & 24.0 & 0.2 & 3.31 & 0.60 & 0.657 & 92 & 6 & 0.074 & & \\
\hline DP19903 & 5 & 5 & 0.09 & 45.19 & 0.51 & 2.08 & 7.64 & 23.7 & 0.241 & 3.26 & 0.66 & 0.661 & 0.94 & 5 & 0.074 & 0.047 & 94 \\
\hline & 5 & 5 & 0.12 & 45.22 & 0.58 & 2.13 & 7.66 & 23.7 & 0.232 & 3. & 0.70 & 0.672 & 0.94 & 6.01 & 0.076 & & \\
\hline DP19904 & 5 & 5 & 0.28 & 45.38 & 0.47 & 2.15 & 7.71 & 23.9 & 0.243 & 3. & 0.66 & 0.652 & 0.99 & 6. & 0.075 & & \\
\hline DP19905 & 5 & 5 & 0.34 & 45.44 & 0.46 & 2.20 & 7.80 & 23.9 & 0.241 & 3. & 0.63 & 0.637 & 1.06 & 6.02 & 0.075 & 0.046 & \\
\hline DP19906 & 5 & 5 & 0.39 & 45.49 & 0.44 & 2.21 & 7. & 23.7 & 0.225 & 3.0 & 0.61 & 0.615 & 1.06 & 5.99 & 0.073 & 0.051 & 97 \\
\hline & 5 & 5 & 1.39 & & 0.43 & 2.24 & & 23.4 & 0.292 & 2. & 0.75 & 0.571 & 1.11 & 6. & 0.074 & 0.056 & 99 \\
\hline DP19908 & 3 & 6 & 0.10 & 46.70 & 0.58 & 2.20 & 7.79 & 23.4 & 0.315 & 2.88 & 0.76 & 0.612 & 1.12 & 6.32 & 0.073 & 0.053 & \\
\hline
\end{tabular}


Table 3. (Continued).

\begin{tabular}{|c|c|c|c|c|c|c|c|c|c|c|c|c|c|c|c|c|c|}
\hline Lab ID & ڤ్ & 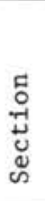 & 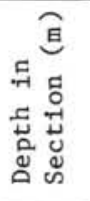 & 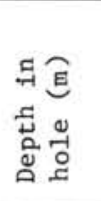 & $\mathrm{Na}$ & $\mathrm{Mg}$ & Al & Si & $\mathrm{P}$ & $\mathrm{K}$ & $\mathrm{Ca}$ & $\mathrm{Ti}$ & $\mathrm{Mn}$ & $\mathrm{Fe}$ & $\mathrm{Ba}$ & $\mathrm{S}$ & $\mathrm{H}_{2} \mathrm{O}$ \\
\hline 09 & 5 & 6 & .24 & .84 & 52 & 09 & 69 & 1 & 303 & 2.91 & 76 & 0.681 & .03 & 6 & 71 & 56 & 101 \\
\hline DP19910 & 5 & 6 & .89 & .49 & 7 & & 7 & 2 & 555 & & .37 & 575 & 3 & & 8 & & 88 \\
\hline DP19911 & 5 & 6 & 1.08 & 7.68 & .37 & 2.18 & 7.16 & 22.9 & 0.557 & 3.09 & 1.44 & 0.578 & 1.46 & .90 & 0.078 & 0.074 & \\
\hline DP19912 & 5 & 6 & 1.29 & 47.89 & 0.37 & 2.14 & 7.13 & 22.8 & .639 & 3.12 & 1.63 & 0.530 & 1.44 & .72 & 0.080 & 0.079 & 114 \\
\hline DP19913 & 5 & 6 & 1.39 & 47.99 & 0.54 & 2.13 & 7.14 & 22.7 & .641 & 3.07 & 1.57 & 0.585 & 1.33 & 6.01 & 0.075 & 0.086 & \\
\hline DP19914 & 5 & 6 & 1.49 & 48.09 & 0.44 & 2.12 & 7.18 & 22.7 & 0.711 & 3.08 & 1.70 & 0.590 & 1.29 & .01 & 0.076 & 0.092 & 111 \\
\hline DP19915 & 5 & 7 & 0.15 & 48.25 & 0.53 & 2.11 & 7.15 & 22.5 & 0.741 & 3.04 & 1.79 & 0.582 & 1.29 & 5.95 & 0.077 & 0.091 & \\
\hline DP19916 & 5 & 7 & 0.24 & 48.34 & 0.46 & 2.06 & 7.19 & 22.6 & 0.759 & 3.06 & 1.81 & 0.584 & 1.27 & & 0.076 & 0.095 & 114 \\
\hline DP19917 & 5 & 7 & 0.28 & 48.38 & 0.42 & 2.08 & 7.19 & 22.6 & 0.756 & & 1.82 & 0.564 & 1.29 & & 0.075 & 0.095 & \\
\hline 18 & 5 & 7 & 0.49 & 59 & 0.47 & 2.07 & 7.2 & 22.7 & & & & & & & & & 109 \\
\hline DP1 & 5 & $\mathrm{C}$ & & 48.70 & 0.36 & 2. & 7. & & & & & & & & & & \\
\hline DP19919 & 6 & 1 & 0.24 & 48.49 & 0.44 & 2.02 & 7.18 & 2 & & & & & & & & 0 & 131 \\
\hline DP19920 & 6 & 1 & 0.68 & 48.93 & 0.66 & 2.09 & 7.18 & 22.7 & 0.781 & 3.11 & 1.94 & 0.479 & 1.31 & 5.68 & 0.074 & 0.0 & \\
\hline 921 & 6 & 1 & 0.72 & 48.97 & 0.50 & 2.05 & 7.06 & 22.3 & 0.871 & 3.08 & 2.05 & 0.495 & 1.26 & 5.71 & 0.072 & 0.109 & \\
\hline DP1 & 6 & 1 & 0.89 & 49.14 & 0.37 & 2.08 & 7.08 & 22.5 & 0.890 & 3.13 & 2.13 & 0.469 & 1.34 & 5.57 & 0.074 & 0.106 & 122 \\
\hline DP & 6 & 1 & 1.00 & 49.25 & 0.47 & 2.07 & 7.07 & 22.5 & 0.913 & 3.20 & 2.19 & 0.4 & 1.35 & & 0.072 & & \\
\hline Df & 6 & 2 & 0.24 & 9 & 0.36 & 2.06 & 6.77 & 22.3 & 0.758 & & 1.91 & 0.4 & 1.35 & & 0.072 & 0.099 & 120 \\
\hline DP & 6 & 2 & 0.28 & 3 & 0.36 & 2.10 & 6.84 & 22.6 & 2 & & 8 & 0.4 & & & 1 & & \\
\hline DP1 & 6 & 2 & 0.35 & 50. & 0 & 2. & 7. & 22 & & & & & & & & & \\
\hline DP & 6 & 2 & 0.49 & 50.24 & 0.36 & 2. & 7. & 2 & & & & & & & & & 108 \\
\hline 28 & 6 & 2 & 1.20 & 50.95 & 0.55 & 2.05 & 7.28 & 22.8 & 0.841 & .38 & 2.12 & 0. & 1. & 8 & 0.075 & 0. & \\
\hline 29 & 6 & 2 & 1.39 & 51.14 & 0.42 & 2.09 & 7.17 & 22.6 & 0.870 & .28 & 2.18 & 0.390 & 1.3 & .17 & 0.076 & 0.104 & 104 \\
\hline & 6 & 2 & 1.42 & 51.17 & 0.45 & 2.17 & 7.24 & 22.9 & 0.753 & 3.34 & 2.18 & 0.392 & 1.32 & 5.19 & 0.077 & 0 . & \\
\hline DP & 6 & 3 & 0.18 & 51.43 & 0.40 & 2.08 & 7.13 & 22.5 & 0.825 & 3.23 & 2.07 & 0.3 & 1. & 5.27 & 0.074 & 0 . & \\
\hline 31 & 6 & 3 & 0.24 & & 0 & 2.10 & 7. & 22.7 & 0.8 & 5 & 2.04 & 0.3 & 1. & 5.30 & 0.079 & 0. & \\
\hline DP & 6 & 3 & 0.37 & & 0 & 2 & 7. & 22.6 & & & 1.93 & & & & & & 104 \\
\hline DP1 & 6 & 3 & 0.56 & 51.81 & 0. & 2 & 7 & 2 & 3 & & & & & & & & \\
\hline 934 & 6 & 3 & 0.64 & 51.89 & 0.44 & 2.14 & 7.2 & 22.9 & r. & 3 & 1.8 & 0. & 1. & 1 & 0. & 0. & 102 \\
\hline DP1 & 6 & 3 & 1.00 & 52.25 & 0.41 & 2.12 & 7.12 & 22.6 & 0.840 & .26 & 2.15 & 0.387 & 1.30 & 5.18 & 0.076 & 0.1 & \\
\hline 36 & 6 & 3 & 1.16 & 52.41 & 0.37 & 2.15 & 7.23 & 22.9 & 0.782 & .32 & 1.97 & 0.390 & 1. & 5.18 & 0.075 & 0.097 & 106 \\
\hline 37 & 6 & 3 & 1.29 & 52.54 & 0.4 & 2.12 & 7.17 & 22.6 & 0.769 & 3.26 & 1.91 & 0.3 & 1.33 & 5.28 & 0.076 & 0. & \\
\hline & 6 & 3 & 1.39 & 52.64 & 0.37 & 2.11 & 7.1 & 22.6 & 0.7 & 3.27 & 1.88 & 0.3 & 1. & 7 & 5 & 0 . & 101 \\
\hline & 6 & 4 & 0.04 & 52.79 & 0.47 & 2.15 & 7. & 22.8 & 0.7 & 3.25 & 1.76 & 0.3 & 1. & 34 & 0.075 & 0 . & \\
\hline & 6 & 4 & 0.13 & 52 & & & 7. & 23.2 & & 8 & 5 & & & & & & 103 \\
\hline 41 & 6 & 4 & 1.04 & 53.79 & & 2. & & 2 & & & 9 & & & & & & \\
\hline DP19942 & 6 & 4 & 1.09 & 53.84 & 0.1 & 2.12 & 7. & 4 & 5 & & 0 & & 1. & 1 & 0. & 0. & \\
\hline DP19943 & 6 & 4 & 1.29 & 54.04 & 0.42 & 2.11 & 7.3 & 23.4 & 85 & 3.45 & 1.03 & 0. & 1. & 5.20 & 0.077 & 0. & 101 \\
\hline 944 & 6 & 5 & 0.03 & 54.28 & 0.40 & 2.12 & 7.42 & 23.4 & 0.344 & 3.45 & 0.96 & 0.393 & 1. & 5.32 & 0.076 & 0. & \\
\hline DP & 6 & 5 & 0.14 & 54.39 & 0.43 & 2.14 & $7 \cdot 34$ & 23.2 & 0.437 & 3.47 & 1.15 & 0.3 & 1. & 5.22 & 0.075 & 0.080 & 102 \\
\hline & 6 & 5 & 1.06 & 1 & 0.49 & 2.2 & 6.8 & 22.7 & 0.5 & 3.56 & 1.50 & 0. & 2. & .83 & 37 & 0. & \\
\hline & 6 & 5 & 3 & & & & 5. & 18.2 & 0.378 & 2.85 & 8.76 & 1 & & 36 & 9 & & \\
\hline 47 & 6 & 5 & 1.17 & 55. & 9 & & 2 & 6.4 & & 1 & 29. & & & & & & \\
\hline DP19948 & 6 & 5 & 1.29 & 55.54 & 7 & 2.24 & 6 & 22.7 & 3 & 9 & 5 & & & 1 & 58 & 7 & 95 \\
\hline DP19949 & 6 & 6 & 0.10 & 55.85 & .25 & 0.43 & 0.7 & 2.5 & 0 & & 34 & 0 & 0.30 & 0 & 0 , & 8 & \\
\hline DP1 & 6 & 6 & 0.19 & 55.94 & 0.25 & 0.43 & 0.76 & 2.5 & 0.038 & 0.33 & 35. & 0.066 & 0. & 1.11 & 0.057 & 0.016 & 45 \\
\hline 51 & 6 & 6 & 1.38 & 57.13 & 0.24 & 0.83 & 1.6 & 5.8 & 0.098 & 0.75 & 30.12 & 0.127 & 0. & 2.41 & 0.069 & 0. & \\
\hline DP & 6 & 6 & 1.40 & $5^{\prime}$ & 0.3 & 0.80 & 1.67 & 5.7 & 0.094 & 0.74 & 30.55 & 0. & 0. & 2.35 & 0.068 & 0 . & \\
\hline 953 & 6 & 6 & 1.49 & 57 & .26 & 1.31 & 3.43 & 11.5 & 0.159 & 1.71 & 19.61 & 0.2 & 1. & 4.21 & 5 & 4 & 79 \\
\hline & 6 & 7 & 0.24 & 57 & 0.3 & 2.29 & 6 & 23.1 & 0.381 & 3.23 & 1.01 & 0.3 & 1. & 6.79 & 0. & 0. & 110 \\
\hline & 6 & 7 & 0.28 & & 0 . & 2. 30 & 6. & 23.8 & 0.313 & 3.38 & 3 & & & 02 & & & \\
\hline DP & 6 & 7 & 0.42 & 67 & 0. & 0.48 & 0.84 & 2.8 & 0.039 & 0.37 & 34.44 & 0.072 & 0.34 & 1.58 & 0.059 & 0.019 & \\
\hline DP19957 & 6 & 7 & 0.51 & & 3 & 2.33 & 6.71 & 22.8 & 0.288 & 3.19 & 0.82 & 0.351 & 1.67 & 7.50 & 0.085 & 0.0 & 117 \\
\hline DP1 & 7 & 1 & 0.24 & 58,19 & & 0. & 2. & 7. & & 1.01 & 26. & & 0 . & & 0. & & 87 \\
\hline 59 & 7 & 1 & 0.49 & 58.44 & 0.20 & 0.96 & 2.28 & 7.7 & 0.125 & 1.15 & 24.88 & 0.190 & 0.7 & 3.38 & 0.071 & 0.030 & 81 \\
\hline DP18575 & 7 & 1 & 0.90 & 58.85 & 0.00 & 0.43 & 0.5 & 2.1 & $0 \Omega 31$ & 0.25 & 36.48 & 0.058 & 0 & 0.79 & 0.055 & 0.033 & \\
\hline 60 & 7 & 1 & 1.13 & & 2 & & 0.56 & 2.0 & 0.030 & 0.25 & 35.84 & 0.057 & 0 . & 0.76 & 0.059 & 0.0 & \\
\hline 61 & 7 & 1 & 1.33 & 59.28 & 0.19 & 0.39 & 0.54 & 2.0 & 0.030 & 0.24 & 35.90 & 0.056 & 0. & 0.75 & 0.057 & 0.0 & \\
\hline & 7 & 1 & 1.39 & & 0.21 & 0.39 & 0.54 & 2.0 & 0.031 & 0.24 & 36.11 & 0.056 & 0. & 0.75 & 0.059 & & 50 \\
\hline & 7 & 2 & 0.04 & & 0.29 & 0.40 & 0.57 & 2.1 & 0.031 & 0.25 & 36.07 & 0.056 & 0.20 & 0.77 & & & \\
\hline & 7 & 2 & 0.24 & 69 & 0.16 & 0.38 & 0.51 & 1.9 & 0.029 & 0.23 & 36.18 & 0.054 & 0.19 & 0.73 & 0.057 & 0.016 & נכ \\
\hline & 7 & 2 & 1.35 & & 0. & 0. & 0.53 & 2.0 & 0.029 & 0.23 & 35.94 & 0.054 & 0.19 & 0.73 & 0.057 & 0.015 & \\
\hline & 7 & 2 & 1.49 & & & & & 1.9 & & 0.23 & & 0.055 & & 0.74 & 0.057 & & 47 \\
\hline DP19967 & 7 & 3 & 0.10 & 61.05 & 0.25 & 0.40 & 0.57 & 2.1 & 0.030 & 0.25 & 35.66 & 0.057 & 0.18 & 0.76 & 0.058 & 0.015 & \\
\hline
\end{tabular}


Table 3. (Continued).

\begin{tabular}{|c|c|c|c|c|c|c|c|c|c|c|c|c|c|c|c|c|c|}
\hline Lab ID & $\begin{array}{l}\stackrel{\nu}{0} \\
\text { ن }\end{array}$ & 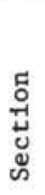 & 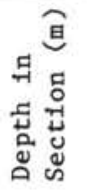 & 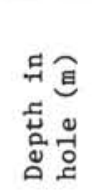 & $\mathrm{Na}$ & $\mathrm{Mg}$ & A1 & S1 & $\mathrm{P}$ & $\mathrm{K}$ & $\mathrm{Ca}$ & $\mathrm{T} 1$ & Mn & $\mathrm{Fe}$ & $\mathrm{Ba}$ & $\mathrm{S}$ & $\mathrm{H}_{2} \mathrm{O}$ \\
\hline 9968 & 7 & 3 & 0.19 & .14 & 0.19 & 0.39 & 6 & 2.1 & 0.0 & 0.25 & 35.57 & 0.057 & 0.18 & 0.75 & 0.057 & 0.017 & 49 \\
\hline 969 & 7 & 3 & 0.38 & . & 0. & & 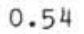 & 2.0 & 0.0 & 0.24 & 35 & 0. & 8 & 0.76 & 9 & 0.016 & \\
\hline & 7 & 3 & 0.45 & 61. & 0.22 & 0.40 & 0.56 & 2.1 & 0.029 & 0.25 & 35.80 & 0.056 & 0.19 & 0.76 & 0.059 & 0.016 & \\
\hline DP19971 & 7 & 3 & 0.59 & 61.54 & 0.20 & 0.39 & 0.54 & 2.0 & 0.030 & 0.24 & 35.93 & 0.055 & 0.16 & 0.72 & 0.057 & 0.017 & 48 \\
\hline & 7 & 3 & 1.32 & 62.27 & 0.25 & 0.54 & 0.87 & 3.7 & 0.144 & 0.42 & 33.49 & 0.096 & 0.10 & 1.11 & 0.071 & 0.033 & \\
\hline DP19972 & 7 & 4 & 0.43 & 62.88 & 0.21 & 0.63 & 1.23 & 4.2 & 0.066 & 0.60 & 31.76 & 0.114 & 0.48 & 1.71 & 0.061 & 0.023 & \\
\hline DP19973 & 7 & 4 & 0.45 & 62.90 & 0.27 & 0.50 & 0.86 & 2.9 & 0.033 & 0.39 & 34.28 & 0.072 & 0.31 & 1.04 & 0.056 & 0.019 & \\
\hline DP19974 & 7 & 4 & 0.54 & 62.99 & 0.21 & 0.42 & 0.64 & 2.2 & 0.027 & 0.29 & 35. & 0.056 & 23 & 0.75 & 6 & 6 & 50 \\
\hline DP19975 & 7 & 4 & 1.0 & 1 & 0 & 0. & 0. & 4 & 0 & 1 & 3 & 1 & 5 & 30 & 7 & 6 & 50 \\
\hline DP19976 & 7 & 5 & 0.4 & 9 & 0. & 2. & 6. & 24.0 & 0. & 3 & 0 & 0.3 & 2 & 1 & 0 . & 0. & \\
\hline DP19977 & 7 & 5 & 0.64 & 6 & 0.21 & 0.35 & 0.1 & 1.7 & 0.024 & 0.21 & 36.13 & 0.048 & 0.17 & 0.53 & 0.055 & 0.016 & 49 \\
\hline 577 & 7 & 5 & 1.13 & 65.08 & 0.02 & 0.44 & 0.66 & 2.5 & 0.046 & 0.31 & 36.68 & 0.066 & 0.18 & 0.78 & 0.066 & 0.034 & \\
\hline DP19978 & 7 & 5 & 1.27 & 65.22 & 0.27 & 0.37 & 0.5 & 2.0 & 0.025 & 0.25 & 36.17 & 0.051 & 0.21 & 0.63 & 0.054 & 0.015 & \\
\hline 979 & 7 & 5 & 1.39 & 65.34 & 0.24 & 0.37 & 0.54 & 1.9 & 0.026 & 0.24 & 35.96 & 0.051 & 0.21 & 0.63 & 0.055 & 0.015 & 46 \\
\hline DP19980 & 7 & 6 & 0.18 & 65.63 & 0.15 & 0.40 & 0.58 & 2.1 & 0.027 & 0.27 & 35.97 & 0.055 & 0.22 & 0.67 & 0.071 & 0.023 & \\
\hline DP19981 & 7 & 6 & 0.21 & 6 & 0.30 & 0.38 & 0.57 & 2.0 & 0.028 & 0.25 & 35.75 & 0.053 & 0.21 & 0.65 & 0.074 & 0.018 & \\
\hline 82 & 7 & 6 & 0.29 & & 0.26 & 0.40 & 0.64 & 2.3 & 0.027 & 0.29 & 35 & 0.0 & 0.23 & 2 & & & 47 \\
\hline DP1 & 7 & 6 & 1.14 & 6 & 0.25 & 0.30 & 0.35 & 1.4 & 0.025 & 0.16 & 36.65 & 0.043 & 0.14 & 0.44 & 0.055 & 0. & 48 \\
\hline DP19984 & 7 & 6 & 1.18 & 3 & 0.21 & 0.32 & 0.40 & 1.5 & 0.0 & 0.18 & 0 & 0.046 & 0.15 & 0.49 & 0. & 0.018 & \\
\hline 985 & 7 & 6 & 1.39 & & 0.21 & 0.32 & 0.40 & 1.5 & 0.025 & 0.18 & 36.69 & 0.046 & 0.15 & 0.49 & 0.056 & 0.017 & 44 \\
\hline 86 & 7 & 7 & 0.05 & 0 & 0.27 & 0.32 & 0.39 & 1.5 & 0.025 & 0.18 & 36. & 0.045 & 0.14 & 0.48 & 0.0 & 0.018 & \\
\hline DP19987 & 7 & 7 & 0.19 & & 0.19 & 0.34 & 0.43 & 1.6 & 0.028 & 0.20 & 36.50 & 0.049 & 0.15 & 0.53 & 0.066 & 0.018 & 45 \\
\hline 988 & 7 & 7 & 0.55 & & 0.57 & 2.60 & 6.44 & 24.3 & 0.291 & 3.15 & 0.83 & 0.364 & 1.21 & 5.40 & 0.086 & 0.045 & \\
\hline & 7 & 7 & .69 & & 0.27 & 1.00 & 2.12 & . & 2 & & 25.76 & 0.177 & 0.66 & 2.73 & 0.068 & 0.031 & 48 \\
\hline
\end{tabular}

Note: Depth in hole omitted for disturbed samples. 
Table 4. Elemental abundance (wt. \%) and water content (\% dry wt.) of Hole 576B samples.

\begin{tabular}{|c|c|c|c|c|c|c|c|c|c|c|c|c|c|c|c|c|c|}
\hline Lab ID & రુ & 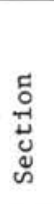 & 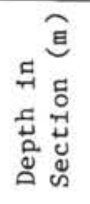 & 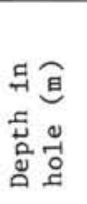 & $\mathrm{Na}$ & $\mathrm{Mg}$ & Al & Si & $\mathrm{P}$ & K & $\mathrm{Ca}$ & $\mathrm{T} 1$ & Mn & $\mathrm{Fe}$ & $\mathrm{Ba}$ & $\mathrm{S}$ & $\mathrm{H}_{2} \mathrm{O}$ \\
\hline 19212 & 1 & 1 & 0.06 & 0.06 & 0.37 & 1.75 & 7.20 & 26.8 & 0.049 & 2.51 & 0.40 & 0.407 & 0.24 & 4.66 & 0.095 & 0.031 & 169 \\
\hline 9213 & 1 & 1 & .16 & 16 & & 1.74 & 7.15 & & 0.049 & 2.51 & 0.40 & 0.400 & 0.28 & 4.57 & .093 & .040 & 179 \\
\hline 19214 & 1 & 1 & 0.26 & 26 & 0.28 & 1. & 7.18 & 27.2 & 0.048 & 2.51 & 0.43 & 0.405 & 0.33 & 4.65 & 0.099 & & 177 \\
\hline 19215 & 1 & 1 & 0.36 & 0.36 & 0.24 & 1.70 & 7. & 27 & & & 0.46 & 0.393 & 0.31 & 4.62 & .105 & .040 & 184 \\
\hline DP19216 & 1 & 1 & 0.46 & 0.46 & 0.42 & 1.77 & 7.29 & 26.5 & 0.053 & 2.40 & 0.66 & 0.410 & 0.45 & 4.87 & .094 & .039 & 149 \\
\hline DP19217 & 1 & 1 & 0.56 & 0.56 & 0.50 & 1.84 & 7.53 & 26.7 & 0.054 & 2.46 & 0.77 & 0.434 & 0.50 & 5.08 & 0.097 & 0.050 & 134 \\
\hline DP19218 & 1 & 1 & 0.66 & 0.66 & 0.48 & 1.87 & 7.57 & 26.4 & 0.054 & 2.61 & 0.47 & 0.425 & 0.65 & 4.85 & 0.098 & .042 & 132 \\
\hline 19219 & 1 & 1 & 0.76 & 0.76 & 0.46 & 1.88 & 7.66 & 26.7 & 0.053 & 2.68 & 0.43 & 0.438 & 0.24 & 4.93 & 0.093 & 0.038 & 130 \\
\hline 19220 & 1 & 1 & 0.86 & 0.86 & 0.43 & 1.90 & 7.64 & 26.4 & 0.051 & 2.67 & 0.41 & 0.425 & 0.28 & 4.87 & 0.089 & 0.037 & 137 \\
\hline 19221 & 1 & 1 & 0.96 & 0.96 & 0.46 & 1.85 & 7.61 & 26 & 0.052 & 2.62 & 0.46 & 0.426 & 0.36 & 4.87 & .087 & .033 & 137 \\
\hline 19222 & 1 & 1 & 1.06 & 1.06 & 0.43 & 1.85 & 7. & & 55 & & 0.48 & & 47 & 4.87 & 8 & .032 & 150 \\
\hline DP19223 & 1 & 1 & 1.16 & 1.16 & 0.43 & 1.84 & 7.54 & 26.4 & 0.053 & 2.57 & 0.48 & 0.425 & 0.52 & 4.84 & 0.100 & .041 & 145 \\
\hline DP19224 & 1 & 1 & 1.26 & 1.26 & 0.39 & 1.82 & 7.46 & 26.2 & 0.054 & 2.54 & 0.44 & 0.407 & 0.58 & 4.75 & 0.106 & 0.043 & 146 \\
\hline DP19225 & 1 & 1 & 1.36 & 1.36 & 0.42 & 1.87 & 7.52 & 26.5 & 0.055 & 2.55 & 0.44 & 0.419 & 0.83 & 4.75 & 0.103 & 40 & 146 \\
\hline DP19226 & 1 & 1 & 1.46 & 1.46 & 0.44 & 1.84 & 7.41 & 26.4 & 0.055 & 2.55 & 0.47 & 0.413 & 0.49 & 4.74 & 0.096 & 48 & 136 \\
\hline DP1 & 1 & 2 & 0.06 & 1.56 & 0.40 & 1.88 & 7.40 & 26.5 & 0.053 & 2.60 & 0.43 & 0.422 & 0.37 & 4.78 & 97 & 43 & 138 \\
\hline 19228 & 1 & 2 & 0.16 & 1.66 & 0.38 & 1.85 & $7 \cdot 34$ & 26.9 & 0.052 & 2.56 & 0.45 & 0.4 & 0. & 4. & 5 & 35 & 155 \\
\hline 29 & 1 & 2 & 0.26 & 1.76 & 0.16 & 1.69 & 6. & 2 & 0 & 2 & 0 & 3 & 7 & 1 & & 31 & 200 \\
\hline 30 & 1 & 2 & 0.36 & 1.86 & 0.16 & 1.67 & 6.70 & 27.8 & 0.050 & 2.35 & 0.25 & 0.374 & 0.51 & 4.31 & 5 & 0.012 & 219 \\
\hline DP19231 & 1 & 2 & 0.46 & 1.96 & 0.28 & $1.7:$ & 6.82 & 27.6 & 0.050 & 2.36 & 0.23 & 0.374 & 0.61 & 4.30 & 0.103 & 0 & 226 \\
\hline DP19232 & 1 & 2 & 0.56 & 2.06 & 0.27 & 1.70 & 6.74 & $27 \cdot 3$ & 0.050 & 2.37 & 0.25 & 0.371 & 0.47 & 4.27 & 0. & 0 & 217 \\
\hline DP19233 & 1 & 2 & 0.66 & 2.16 & 0.11 & 1.79 & 6.98 & 27.7 & 0.049 & 2.50 & 0.30 & 0.393 & 0.33 & 4.46 & 0.099 & 0.037 & 228 \\
\hline 234 & 1 & 2 & 0.76 & 2.26 & 0.13 & 1.75 & 6.82 & 27.5 & 0.0 & 2.45 & 0.36 & 0.386 & 0.28 & 4.47 & 0.0 & 35 & 214 \\
\hline DP19237 & 1 & 2 & 0.86 & 2.36 & 0.40 & 1.77 & 7. & 26.9 & $0.8-1$ & 2. & 0.37 & 7 & 4 & 4.66 & 6 & 7 & 195 \\
\hline DP19238 & 1 & 2 & 0.96 & 2.46 & 0.1 & 1.76 & 6. & 27. & 0. & 2. & 3 & 5 & 4 & 4. & 7 & 21 & 225 \\
\hline DP19235 & 1 & 2 & 1.06 & 2.56 & 0.17 & 1.76 & 7.01 & 27.5 & 0.049 & 2.53 & 0.26 & 0.389 & 0.12 & 4.53 & 0.097 & 0.0 & 210 \\
\hline DP19236 & 1 & 2 & 1.16 & 2.66 & 0.29 & 1.77 & 7.05 & 26.9 & 0.048 & 2.53 & 0.33 & 0.391 & 0.13 & 4.52 & 0. & 30 & 201 \\
\hline DP19239 & 1 & 2 & 1.26 & 2.76 & 0.29 & 1.76 & 7.25 & 27.0 & 0.050 & 2.52 & 0.37 & 0.407 & 0.12 & 4.64 & 0.093 & 22 & 188 \\
\hline DP19240 & 1 & 2 & 1.36 & 2.86 & 0.32 & 1.75 & 7.23 & 26.8 & 0.050 & 2.51 & 0.34 & 0. & 0.18 & 4.59 & 2 & 25 & 181 \\
\hline 41 & 1 & 2 & 1.46 & 2.96 & 0.10 & 1.66 & 6.89 & 27.3 & 0.049 & 2. & 0.22 & 0 . & 0.17 & 4.33 & 7 & 6 & 252 \\
\hline DP19242 & 1 & 3 & 0.06 & 3.06 & 0.00 & 1.62 & 6.69 & $27 \cdot 3$ & 0.0 & 2. & 0. & 0. & 0.20 & 9 & 1 & 9 & 274 \\
\hline DP19243 & 1 & 3 & 0.1 & 3. & 0 & 1.74 & 7. & 26 & 3 & 8 & 8 & 0. & 26 & 0 & 39 & 33 & 189 \\
\hline DP19244 & 1 & 3 & 0.26 & 3.26 & 0 & 1. & 7. & 6 & 53 & & 5 & 1 & 3 & 1 & 3 & 0 & 185 \\
\hline DP19245 & 1 & 3 & 0.36 & 3.36 & 0.36 & 1.76 & 7.31 & 26.5 & 0.054 & 2. & 0.41 & 0. & 0.34 & 4.64 & 0.089 & 0. & 166 \\
\hline DP19246 & 1 & 3 & 0.47 & 3.47 & 0.35 & 1.78 & 7.32 & 26.7 & 0.055 & 2.55 & 0.43 & 0.406 & 0.42 & 4.66 & 0.091 & & 165 \\
\hline DP19247 & 1 & 3 & 0.56 & 3.56 & 0.39 & 1.76 & 7.26 & 26.4 & 0.054 & 2.54 & 0.44 & 0.403 & 0.34 & 4.66 & 0.093 & 0.051 & 153 \\
\hline DP19248 & 1 & 3 & 0.66 & 3.66 & 0.43 & 1.78 & $7 \cdot 31$ & 26.2 & 0.054 & 2.56 & 0.42 & 0.402 & 0.45 & 4.65 & 0.091 & 42 & 150 \\
\hline 249 & 1 & 3 & 0.76 & 3.76 & 0.43 & 1.77 & 7.3 & .3 & 0 & & 0.42 & 0. & 0.37 & 0 & 0. & 33 & 144 \\
\hline DP19250 & 1 & 3 & 0.86 & 3.86 & 0.38 & 1.81 & 7. & 5.9 & 0.053 & 2. & 0 & 4 & 33 & 2 & 4 & 30 & 152 \\
\hline DP19251 & 1 & 3 & 0.96 & 3.96 & 0.40 & 1.79 & 7.44 & 26.2 & 5 & 2. & 0.43 & 0. & 0.34 & 4.70 & 0. & 0. & 157 \\
\hline DP19252 & 1 & 3 & 1.06 & 4.06 & 0.37 & 1.79 & 7.3 & 26.1 & 0.057 & 2.56 & 0.46 & 0.404 & 0.43 & 4.60 & 0.087 & 0.040 & 160 \\
\hline DP19253 & 1 & 3 & 1.16 & 4.16 & 0.34 & 1.78 & 7.42 & 26.1 & 0.056 & 2.53 & 0.44 & 0.403 & 0.38 & 4.62 & 0.088 & 0.031 & 152 \\
\hline DP19254 & 1 & 3 & 1.26 & 4.26 & 0.42 & 1.74 & 7.28 & 26.1 & 0.057 & 2.56 & 0.50 & 0.395 & 0.48 & 4.46 & 0.092 & 0.040 & 141 \\
\hline DP19255 & 1 & 3 & 1.36 & 4.36 & 0.39 & 1.74 & 7.33 & 26.5 & 0.054 & 2.60 & 0.51 & 0.394 & 0.27 & 4.50 & 0.095 & 0.046 & 144 \\
\hline DP19256 & 1 & 3 & 1.46 & 4.46 & 0.38 & 1.81 & 7.52 & 26.3 & 0.058 & 2.55 & 0.47 & 0.409 & 0.47 & 4.68 & 0.091 & 0.032 & 151 \\
\hline & 1 & 4 & 0.06 & 4.56 & 0.37 & 1.78 & 7.4 & 26.1 & 0.058 & 2.55 & 0.50 & 0. & 0.48 & 4.61 & 0. & & 144 \\
\hline 9258 & 1 & 4 & 0.16 & 4.66 & 0.44 & & & & 0.0 & & 0.52 & & & 4.47 & 4 & 0. & 140 \\
\hline 59 & 1 & 4 & 0.26 & 4.76 & 0.35 & 1.88 & 7.41 & 25.8 & 0.054 & 2. & 0.44 & 0.412 & 0.30 & 4.75 & 0. & 0.055 & 148 \\
\hline DP19260 & 1 & 4 & 0.36 & 4.86 & 0.33 & 1.84 & 7.36 & 26.0 & 0.053 & 2.5 & 0.43 & 0.402 & 0.31 & 4.66 & 0.093 & 44 & \\
\hline DP19261 & 1 & 4 & 0.46 & 4.96 & 0.37 & 1.89 & 7.52 & 26.3 & 0.054 & 2.62 & 0.43 & 0.422 & 0.36 & 4.81 & 0.099 & 0.050 & 144 \\
\hline DP19262 & 1 & 4 & 0.56 & 5.06 & 0.33 & 1.91 & 7.52 & 26.3 & 0.053 & 2.65 & 0.42 & 0.422 & 0.29 & 4.81 & 0.099 & 0.055 & 145 \\
\hline DP19263 & 1 & 4 & 0.66 & 5.16 & 0.33 & 1.91 & 7.48 & 26.0 & 0.052 & 2.63 & 0.39 & 0.420 & 0.23 & 4.84 & 0.092 & 0.040 & 152 \\
\hline & 1 & 4 & 0.76 & 5.26 & 0.31 & 1.92 & 7.5 & 26.0 & 0.054 & 2.65 & 0.43 & & & 4.79 & & & 151 \\
\hline & 1 & 4 & 0.86 & 5.36 & 0.35 & 1.89 & 7.48 & 26.1 & 0.056 & 2.62 & 0.43 & 0.413 & 0.28 & 4.80 & 0.089 & 0.050 & 143 \\
\hline 66 & 1 & 4 & 0.96 & 5.46 & 0.35 & 1.92 & 7.56 & 26.2 & 0.053 & 2.64 & 0.41 & 0.421 & 0.24 & 4.83 & 0.090 & 0.042 & 153 \\
\hline 9267 & 1 & 4 & 1.06 & 5.56 & 0.35 & 1.9 & 7.55 & 26.0 & 0.052 & 2.65 & 0.41 & 0.414 & 0.24 & 4.82 & 0.093 & 0.042 & 144 \\
\hline DP19268 & 1 & 4 & 1.16 & 5.66 & 0.37 & 1.97 & 7.69 & 26.4 & 0.052 & 2.70 & 0.42 & 0.438 & 0.24 & 4.93 & 0.097 & 0.047 & 142 \\
\hline DP19269 & 1 & 4 & 0.26 & 5.76 & 0.37 & 1.94 & 7.62 & 26.1 & 0.051 & 2.68 & 0.43 & 0.429 & 0.23 & 4.87 & 0.091 & 0.047 & 139 \\
\hline DP19270 & 1 & 4 & 1.36 & 5.86 & 0.42 & 1.93 & 7.61 & 26.0 & 0.051 & 2.68 & 0.45 & 0.428 & 0.26 & 4.83 & 0.093 & 0.058 & 138 \\
\hline DP19271 & 1 & 4 & 1.46 & 5.96 & 0.38 & 1.93 & 7.63 & 26.1 & 0.052 & 2.66 & 0.46 & 0.429 & 0.28 & 4.84 & 0.092 & 0.055 & 136 \\
\hline 9278 & 1 & 5 & 0.06 & 6.06 & 0.38 & 1.92 & 7.73 & 26.3 & 0.054 & 2.65 & 0.46 & 0.433 & 0.34 & 4.90 & 0.091 & 0.050 & 139 \\
\hline & 1 & 5 & 0.16 & 6.16 & 0.39 & & 7.59 & 26.0 & 0.055 & 2.60 & 0.47 & 0.422 & 0.35 & 4.79 & 0.092 & 0.051 & 143 \\
\hline DP19280 & 1 & 5 & 0.26 & 6.26 & 0.36 & 1.90 & 7.67 & 26.2 & 0.054 & 2.63 & 0.44 & 0.422 & 0.32 & 4.82 & 0.093 & 0.047 & 147 \\
\hline
\end{tabular}




\begin{tabular}{|c|c|c|c|c|c|c|c|c|c|c|c|c|c|c|c|c|c|}
\hline Lab ID & ஸ્ّ & 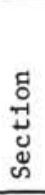 & 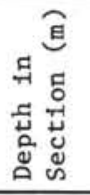 & 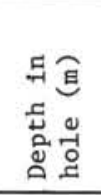 & $\mathrm{Na}$ & $\mathrm{Mg}$ & A1 & Si & $\mathrm{P}$ & $\mathrm{K}$ & $\mathrm{Ca}$ & $\mathrm{T} 1$ & $\mathrm{Mn}$ & $\mathrm{Fe}$ & $\mathrm{Ba}$ & S & $\mathrm{H}_{2} \mathrm{O}$ \\
\hline 9281 & 1 & 5 & 0.36 & 6.36 & 0.32 & 1.88 & .62 & 4 & 55 & 2.61 & 0.47 & 0.419 & 35 & 4.76 & .091 & .048 & 154 \\
\hline 9282 & 1 & 5 & 0.46 & 6.46 & 0.41 & 1.84 & .53 & 26.2 & 0.056 & 2.59 & 0.49 & & & 4.74 & .093 & & 138 \\
\hline DP19283 & 1 & 5 & 0.56 & 6.56 & 0.46 & 1.86 & 7.56 & 26.5 & 0.056 & 2.62 & 0.51 & 0.419 & 0.34 & 4.75 & 0.094 & 0.060 & 133 \\
\hline DP19284 & 1 & 5 & 0.66 & 6.66 & 0.39 & 1.90 & 7.60 & 26.4 & 0.054 & 2.66 & 0.45 & 0.428 & 0.30 & 4.80 & 0.097 & 0.054 & 139 \\
\hline DP19285 & 1 & 5 & 0.76 & 6.76 & 0.41 & 1.91 & 7.58 & 26.4 & 0.054 & 2.65 & 0.46 & 0.427 & 0.31 & 4.79 & 0.096 & 0.054 & 137 \\
\hline DP19286 & 1 & 5 & 0.86 & 6.86 & 0.38 & 1.95 & 7.50 & 26.3 & 0.054 & 2.60 & 0.45 & 0.414 & 0.38 & 4.77 & 0.095 & 0.046 & 152 \\
\hline DP19287 & 1 & 5 & 0.96 & 6.96 & 0.38 & 1.89 & 7.43 & 26.6 & 0.048 & 2.58 & 0.45 & 0.407 & 0.36 & 4.58 & 0.083 & 0.032 & 147 \\
\hline DP1 & 1 & 5 & 1.06 & 7.06 & 0.52 & 1.71 & 7.04 & 26.9 & 0.047 & 2.45 & 0.61 & 0.375 & & & & & 125 \\
\hline 89 & 1 & 5 & 1.16 & 7.16 & 0.47 & 1.74 & 7.16 & 26.7 & 0.045 & 2.65 & 0.48 & 0.371 & 0.27 & 4.12 & 0.085 & 5 & 119 \\
\hline 9290 & 1 & 5 & 1.26 & 7.26 & 0.35 & 1.94 & 7.51 & 26.0 & 0.047 & 2.62 & 0.40 & 0.415 & 0.41 & 4.70 & .089 & & 148 \\
\hline 91 & 1 & 5 & 1.36 & 7.36 & 0.40 & 1.99 & 7.51 & 25.9 & 0.049 & 2.63 & 0.41 & 0.415 & 0.54 & 4.77 & .089 & 056 & 141 \\
\hline DP19292 & 1 & 5 & 1.46 & 7.46 & 0.30 & 1.96 & 7.46 & 25.8 & 0.047 & 2.56 & 0.35 & 0.399 & 0 . & 4.67 & 83 & .035 & 174 \\
\hline DP19272 & 1 & 6 & 0.06 & 7.56 & 0.49 & 1.97 & 7.43 & 25.7 & 0. & 2.66 & 0.43 & 0.408 & 0.42 & 4.73 & 0.093 & 0.069 & 132 \\
\hline DP19273 & 1 & 6 & 0.16 & 7.66 & 0.32 & 2.04 & 7.67 & 26.7 & 0.047 & 2.71 & 0.41 & 0.438 & 0.32 & & 0.0 & 0.062 & 160 \\
\hline DP19274 & 1 & 6 & 0.26 & 7.76 & 0.31 & 1.99 & 7.64 & 26.6 & 0.049 & 2.62 & 0.32 & .426 & .32 & 4.80 & 0.095 & 99 & 177 \\
\hline DP19275 & 1 & 6 & 0.36 & 7.86 & 0.27 & 1.97 & 7.56 & 26.6 & 0.049 & & 0.32 & .413 & 6 & & 3 & 0 & 186 \\
\hline 76 & 1 & 6 & 0.46 & 7.96 & 0.36 & 1.98 & 7.52 & 26.2 & 0.047 & 2.63 & 0.37 & 0.418 & 0.43 & 4. & 3 & & 155 \\
\hline 277 & 1 & 6 & 0.54 & 8.04 & 0.38 & 2.02 & 7.57 & 26.2 & 0.047 & 2.69 & 0.38 & 0.421 & 0.34 & 4.80 & 3 & 4 & 44 \\
\hline 293 & 2 & 1 & 0.11 & 12.11 & 0 & 1.99 & 7.55 & 26.5 & 54 & 2. & 0 & 0.407 & 2 & 4.66 & 9 & 5 & 177 \\
\hline DP19294 & 2 & 1 & 0.19 & 12.19 & 0.31 & 1.99 & 7.56 & 26.3 & 0.052 & 2.62 & 0.38 & 0.417 & 0.39 & 4.73 & 9 & & 171 \\
\hline DP19295 & 2 & 1 & 0.27 & 12.27 & 0.28 & 2.00 & 7.55 & 26.3 & 0.054 & 2.55 & 0.39 & 0.412 & 0.44 & 4.70 & 0.093 & 0.036 & 180 \\
\hline DP1 & 2 & 1 & 0.36 & 12.36 & 0.23 & 1.98 & 7.43 & 26.1 & 0.054 & 2.51 & 0.36 & .402 & 0.44 & 4.63 & 0.0 & 1 & 200 \\
\hline 297 & 2 & 1 & 0.46 & 2.46 & 0. & 1.94 & 7.40 & 26.2 & 0.056 & 2. & & & & & & & 193 \\
\hline 9298 & 2 & 1 & 0.56 & 2.56 & 0.30 & 1.9 & 7.33 & 26.2 & 54 & 2. & 0 & 6 & 0. & 4. & 0 & & 30 \\
\hline 99 & 2 & 1 & 0.66 & 12.66 & 0.28 & 1.98 & 7.56 & 26.3 & 51 & 2. & 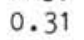 & 14 & 0 . & 4. & & & 181 \\
\hline 300 & 2 & 1 & 0.76 & 12.76 & 0 & 2.0 & 7 & 26 & & & & 6 & & & & & 82 \\
\hline DP19301 & 2 & 1 & 0.86 & 12.86 & 0.19 & 2.01 & 7.54 & 26.1 & 0.054 & 2.53 & 0.31 & 0.405 & 0.47 & 4.66 & 0.094 & 0.035 & 198 \\
\hline DP19302 & 2 & 1 & 0.96 & 12.96 & 0.23 & 2.03 & 7.53 & 26.3 & 0.055 & 2.55 & 0.36 & 0.407 & 0.43 & 4.66 & 0.088 & 0.047 & 194 \\
\hline 303 & 2 & 1 & 1.06 & 13.06 & 0.26 & 1.99 & 7.36 & 26.0 & 0.054 & 2.50 & 0.38 & 0.397 & 0.51 & & 0. & 54 & 182 \\
\hline 304 & 2 & 1 & 1.16 & 16 & 0.30 & 1.96 & 7.32 & 26.0 & 0.055 & 0 & 39 & & 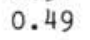 & & & 2 & 181 \\
\hline 305 & 2 & 1 & 1.26 & 13.26 & 0.27 & 1.94 & 7.27 & 25.9 & 53 & 2. & 0 . & 39 & 0.51 & 4. & 0. & 0 . & 183 \\
\hline 306 & 2 & 1 & 1.36 & 36 & 0.26 & 1.97 & 7.31 & 26.3 & 0.054 & 2. & 0. & 0.389 & 0.49 & 4.48 & 0 & 7 & 195 \\
\hline 307 & 2 & 1 & 1.46 & & 0 & & & 26.2 & & 2.57 & 0.31 & 0.402 & 0.41 & 4.56 & .093 & 0.050 & 196 \\
\hline DP19308 & 2 & 2 & 0.06 & & 0.10 & 2.01 & & 26.4 & 0.051 & 2.60 & 0.31 & 0.411 & 0.43 & 4.64 & 0.096 & 0.045 & 193 \\
\hline DP19309 & 2 & 2 & 0.16 & & 0.08 & 1.95 & 7.30 & 26.0 & 0.051 & 2.47 & 0.31 & 0.394 & 0.47 & 4.51 & 0.097 & 0. & 207 \\
\hline DP19310 & 2 & 2 & 0.26 & 13.76 & 0.12 & 1.91 & 7.19 & 25.9 & 0.052 & 2.42 & 0.35 & 0.382 & 0.42 & $u$ & 0.097 & 0.042 & 213 \\
\hline DP19311 & 2 & 2 & 0.36 & 13.86 & 0.14 & 1.95 & 7.31 & 26.4 & 0.052 & 2.46 & 0.41 & 0.400 & 0.39 & 4.59 & 0.091 & 0.047 & 190 \\
\hline 312 & 2 & 2 & 0.46 & & 0.28 & 1.97 & 7.31 & 26.1 & 0.049 & & & & & & & & 160 \\
\hline 313 & 2 & 2 & 0.56 & & 0. & & 7 & 26 & 9 & & & 6 & . & & 1 & 9 & 150 \\
\hline 314 & 2 & 2 & 0.66 & 6 & 0.2 & 1 & 7 & 25 & 0 & $?$ & 0.43 & 4 & 0 & 4 & 0 & 0 & 4 \\
\hline 315 & 2 & 2 & 0.76 & & 0. & & 7. & 26 & & 2 & & 4 & & & 4 & 67 & 158 \\
\hline DP19316 & 2 & 2 & 0.86 & 14.36 & 0.18 & 2.01 & & 26.0 & 0.051 & 2.59 & & 0.410 & 0.38 & 4.64 & 0.103 & 0.052 & 185 \\
\hline DP19317 & 2 & 2 & 0.96 & 14.46 & 0.12 & 2.01 & 7.55 & 26.1 & 0.052 & 2.60 & 0.33 & 0.412 & 0.40 & 4.63 & 0.0 & 0. & 188 \\
\hline DP19318 & 2 & 2 & 1.06 & & 0.20 & 1.96 & 7.34 & 26.0 & 0.053 & 2.58 & 0.38 & 0.390 & 0.42 & & 0.0 & & 174 \\
\hline DP19319 & 2 & 2 & 1.16 & & 0.20 & 2.03 & & 26.2 & 0.053 & 2.62 & & 0.410 & 0.31 & & 0. & & 174 \\
\hline & 2 & 2 & 1.26 & & 0 & 2.0 & 7 & & 0.053 & & & & & 4. & & 4 & 166 \\
\hline & 2 & 2 & 1.36 & & 0.1 & 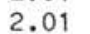 & $\pi$ & 26 & 0.056 & 2. & 0 & 22 & 0 & 4 & 0 & 0 & 172 \\
\hline & 2 & 2 & 1.46 & & & & & & & & & & 0.4 & 7 & & 7 & 194 \\
\hline DP19323 & 2 & 3 & 0.06 & & 0 & 2. & 7. & & & & & 0.404 & 0.45 & 4. & 0. & 0 . & 179 \\
\hline DP19324 & 2 & 3 & 0.15 & 15 & 0.18 & 2.07 & 7.62 & 26.2 & 0.0 & 2.58 & & 0.415 & 0.39 & 4.71 & 0.0 & 0.044 & 178 \\
\hline DP19325 & 2 & 3 & 0.26 & & 0.20 & 2.02 & 7.46 & 26.2 & 0.056 & 2.53 & 0.39 & 0.402 & 0.44 & 4.66 & 0.101 & 0.056 & 179 \\
\hline DP19326 & 2 & 3 & 0.36 & 15.36 & 0.13 & 2.02 & & 26.3 & 0.054 & 2.52 & & 0.414 & 0.46 & & 0.099 & 4 & 173 \\
\hline & 2 & 3 & 0.46 & & 0.1 & 2.01 & & 26.4 & 0.0 & & & & 0.45 & & & & 192 \\
\hline 328 & 2 & 3 & 0.56 & & 0.20 & 2.06 & 7.58 & 26.1 & 0.055 & 2.56 & & 0.414 & 0.51 & 年 & 0. & 0. & 194 \\
\hline DP19329 & 2 & 3 & 0.66 & 15.66 & 0.12 & 2.05 & 7.55 & 26.5 & 0.056 & 2.51 & 0.38 & 0.414 & 0.50 & & 0.098 & & 34 \\
\hline 330 & 2 & 3 & 0.76 & & & & & & & & & & . & & & & 178 \\
\hline DP19331 & 2 & 3 & 0.86 & & 0.15 & 2.0 & & 26.4 & 0. & 2.47 & & 0.417 & 0.50 & & 0. & 0.030 & 193 \\
\hline & 2 & 3 & 1.01 & & 0.21 & 2.02 & & 26.4 & 0.055 & 2.47 & & 0.411 & 0.47 & & 0.096 & 0.031 & \\
\hline & 2 & 4 & 0.16 & & 0.24 & 2.0 & & 26.3 & & & & 0.419 & 0.42 & & & & \\
\hline & 2 & 4 & 0.46 & 16.96 & 0.28 & 2.04 & 7. & 26.2 & 0.052 & 2.63 & & 0.436 & 0.35 & 4.83 & 0.096 & 0.052 & 162 \\
\hline DP19335 & 2 & 4 & 0.56 & 17.06 & 0.24 & 2.03 & & 26.1 & 0.052 & 2.56 & & & 0.40 & & & & 166 \\
\hline & 2 & 4 & 0.66 & & & & & & & & & & & & & & 154 \\
\hline DP1 & 2 & 4 & 0.76 & 17.26 & 0.34 & 2.04 & 7.65 & 26.0 & 0.048 & 2.58 & 0.37 & 0.429 & 0.35 & 4.89 & 0.092 & 0.041 & 156 \\
\hline
\end{tabular}


Table 4. (Continued).

\begin{tabular}{|c|c|c|c|c|c|c|c|c|c|c|c|c|c|c|c|c|c|}
\hline Lab ID & 잉 & 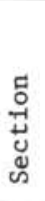 & 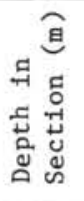 & 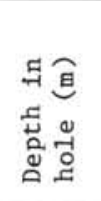 & $\mathrm{Na}$ & $\mathrm{Mg}$ & Al & Si & $\mathrm{P}$ & K & $\mathrm{Ca}$ & Ti & $\mathrm{Mn}$ & $\mathrm{Fe}$ & $\mathrm{Ba}$ & $\mathrm{S}$ & $\mathrm{H}_{2} \mathrm{O}$ \\
\hline 338 & 2 & 4 & 0.86 & 36 & 31 & 0 & 7 & 2 & 50 & 4 & 1 & 8 & 0.38 & 4.82 & 6 & 7 & 155 \\
\hline 339 & 2 & 4 & .96 & & 20 & .06 & & 25.9 & 51 & 2.60 & 0.34 & 0.419 & .43 & 4.75 & .108 & & \\
\hline DP19340 & 2 & 4 & 1.06 & 17.56 & 0.28 & 2.09 & 7.62 & 25.9 & 0.048 & 2.65 & 0.35 & 0.415 & 0.35 & 4.75 & .101 & .060 & 156 \\
\hline DP19341 & 2 & 4 & 1.16 & 17.66 & 0.31 & 2.07 & 7.60 & 25.9 & 0.046 & 2.71 & 0.35 & 0.422 & 0.33 & 4.78 & 0.100 & 0.065 & 146 \\
\hline 19342 & 2 & 4 & 1.26 & 17.76 & 0.33 & 2.06 & 7.70 & 26.2 & 0.045 & 2.74 & 0.33 & 0.428 & 0.31 & 4.85 & 0.105 & 0.055 & 141 \\
\hline 19343 & 2 & 4 & 1.36 & & 0.28 & 2.11 & 7.81 & 26.4 & 0.046 & 2.69 & 0.32 & 0.427 & 0.34 & 4.81 & 0.126 & 0.049 & 155 \\
\hline 344 & 2 & 4 & 1.46 & 17.96 & 0.25 & 2.14 & 7.74 & 26.3 & 0.044 & 2.52 & 0.33 & 0.417 & 0.37 & 4.61 & 0.098 & 0.054 & 173 \\
\hline 345 & 2 & 5 & 0.06 & 18.06 & 0.21 & 2.21 & 7.71 & 26.3 & 0.048 & 2.37 & 0.33 & 0.407 & 0.44 & 4.44 & 0.098 & 0.052 & 180 \\
\hline 346 & 2 & 5 & 0.16 & & 0.20 & & 7.67 & 26. & 0.050 & 2.20 & 0.35 & 0.3 & 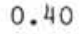 & & 0. & & 193 \\
\hline 347 & 2 & 5 & 0 . & & 0 & 6 & 7 & & 9 & 2. & & 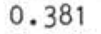 & 6 & & 75 & & 190 \\
\hline DP1 & 2 & 5 & 0.3 & 18 & 0. & 2 & 7 & & & & & & & & & & 9 \\
\hline 349 & 2 & 5 & 0.46 & 18. & 0 & 2 & 7 & & & & & & & & & & 6 \\
\hline 350 & 2 & 5 & 0.56 & 18.56 & 0.35 & 2 & 7 & 2 & 2 & & & & & & & & $\$ 9$ \\
\hline 31 & 2 & 5 & 0.66 & 18.66 & 0.30 & 2.10 & 7.63 & 25.9 & 0.0 & 7 & 0.36 & 0.409 & 0.42 & 4.61 & 0.088 & 53 & 249 \\
\hline 352 & 2 & 5 & 0.76 & 18.76 & 0.38 & 2.10 & 7.82 & 26.3 & 0.051 & 2.67 & 0.39 & 0.434 & 0.42 & 4.87 & 0.093 & & 136 \\
\hline 53 & 2 & 5 & 0.86 & & 0.27 & 2.06 & 7.72 & 26.0 & 0.049 & 2.60 & 0.37 & 0.427 & 0.45 & 4.83 & 0.097 & & 164 \\
\hline 54 & 2 & 5 & 0.96 & & $0.2+2$ & 2.05 & 7.6 & 26. & 0.057 & 2.53 & 0. & 0.4 & 0. & & & & 1 \\
\hline 55 & 2 & 5 & 1. & & 0 & 2. & 0 & 2 & 0 & 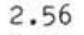 & 0.1 & 0 & 0 & & & & 5 \\
\hline 56 & 2 & 5 & 1. & 19 & 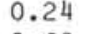 & 2 & 7. & 2 & 9 & & & & & & & & 4 \\
\hline 357 & 2 & 5 & 1.26 & 19.26 & 0.29 & 2.07 & 7.61 & 25.8 & 0 & 2.54 & 0.36 & 0.407 & 0.40 & 4.68 & 0.095 & 9 & 161 \\
\hline 358 & 2 & 5 & 1.36 & 19.36 & 0.33 & 2.03 & 7.78 & 26.0 & 0.0 & 2.68 & 0.34 & 0.423 & 0.42 & 4.91 & 0.099 & 4 & 151 \\
\hline & 2 & 5 & 1.46 & 19.46 & 0.28 & 2.03 & 7.64 & 25.7 & 0.059 & 2.37 & 0.47 & 0.411 & 0.57 & 4.89 & 0.088 & 0. & 181 \\
\hline & 2 & 6 & 0.06 & 19.56 & 0.22 & 2.05 & 7.74 & 25.8 & 0.059 & 2.41 & 0.45 & 0.4 & 0.61 & 4.91 & 0. & 8 & 186 \\
\hline & 2 & 6 & 0.16 & & 0.35 & 2.00 & 7.69 & 25.7 & 0.059 & 2.48 & 0.41 & 0.4 & 0 . & 4. & & 0. & 162 \\
\hline & 2 & 6 & 0.26 & & 0 & 2. & 7.73 & 25.8 & 6 & 2.55 & 0. & & 6 & & & & 1 \\
\hline & 2 & 6 & 0.36 & & 0 & $2+2$ & 7 & 2 & 3 & 54 & 0.1 & 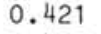 & & & & & 157 \\
\hline DP & 2 & 6 & 0.4 & 19 & 0. & 2. & 7.74 & 2 & & & & & & & & & \\
\hline DP19365 & 2 & 6 & 0.56 & 28 & 0 & 2 & 7. & 2 & 8 & & & & & & & & 245 \\
\hline 366 & 2 & 6 & 0.66 & 20.16 & 0.33 & 2.01 & 7. & $2+2$ & 4 & 2 & 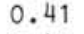 & 0 & 7 & 37 & 39 & 73 & 157 \\
\hline 367 & 2 & 6 & 0.76 & 20.26 & 0.34 & 1.98 & 7.74 & 2 & 8 & 0 & 0.42 & 0.430 & 0.52 & 4.96 & .091 & .059 & 149 \\
\hline 68 & 2 & 6 & 0.86 & 20.36 & 0.35 & 1.99 & 7.76 & 25.6 & 0.056 & 2.56 & 0.46 & 0.437 & 0.53 & 5.05 & 0.0 & 3 & 147 \\
\hline & 2 & 6 & 0.96 & & $0.3 c$ & 1.98 & 7.74 & 25.5 & 0.058 & 2.55 & 0.1 & 0.429 & 0.55 & 5.00 & 0 . & 0. & 162 \\
\hline & 2 & 6 & 1.06 & & .31 & 1. & 7.84 & 25.6 & 0.061 & 2.59 & & 0.4 & 0 & 3 & & & 5 \\
\hline & 2 & 6 & 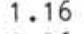 & & & & 7 & & & 2 & & & & & & & \\
\hline DP19372 & 2 & 6 & 1.26 & 20 & 0 & 1. & & & & & & & & & & & \\
\hline 73 & 2 & 6 & 1.36 & 20.86 & 39 & 1.96 & 7.80 & 2 & 0 & 7 & 2 & 1 & 52 & 2 & 35 & 65 & 136 \\
\hline 74 & 2 & 6 & 1.46 & 20.9 & 0.37 & 1.96 & 7.67 & 25.0 & 0. & 2.60 & 2 & 0.1 & 0.60 & 4.89 & 7 & 9 & 147 \\
\hline 75 & 2 & 7 & 0.06 & 21.06 & 0.35 & 1.99 & 7.76 & 25.4 & 0.058 & 2.60 & 0.42 & 0.427 & 0.56 & 4.94 & 0. & 0. & 144 \\
\hline 76 & 2 & 7 & 0.16 & 21.1 & 0.32 & 2.00 & 7.84 & 25.5 & 0.058 & 2.59 & 0.42 & 0.431 & 0.61 & 4.95 & 86 & 59 & 153 \\
\hline 77 & 2 & 7 & 0.26 & 2 & 0.3 & 1.99 & 7.73 & 25.3 & 0.058 & 2.57 & 0.42 & 0.427 & 0. & 4.92 & 0. & 5 & 147 \\
\hline & 2 & 7 & 0.36 & & 0.37 & 2.00 & 7.81 & 25.4 & 0.060 & 2.58 & 0.2 & 0.1 & & & & & 7 \\
\hline & 3 & 1 & 0 . & & & 3 & 7 & 2 & 0 & 2.67 & 0. & 0 & 0 & 9 & & & 141 \\
\hline & 3 & 1 & 0.16 & & & & 7 & & & 7 & 0.3 & & & & & & \\
\hline 81 & 3 & 1 & 0.26 & 21. & 0.33 & & 7 & & & & & & & & & & 144 \\
\hline DP19382 & 3 & 1 & 0.36 & 21.86 & 0.37 & 1.96 & 7.96 & 7 & 66 & 0 & 8 & 0.446 & 0 & 0 & 0. & 0 & 139 \\
\hline DP19384 & 3 & 1 & 0.46 & 21.96 & 0.36 & 1.93 & 7.90 & 25.5 & 0.064 & 2.67 & 0.37 & 0.441 & 0.64 & 4.99 & 0.078 & 0 . & 136 \\
\hline 385 & 3 & 1 & 0.56 & 22. & 0.35 & 1.92 & 7.85 & 25.3 & 0.062 & 2.63 & 0.40 & 0.436 & 0.64 & 5.01 & 0.075 & 0. & 143 \\
\hline DI & 3 & 1 & 0.66 & 22.16 & 0.3 & 1.94 & 7.90 & 25.5 & 0.061 & 2.64 & 0.42 & 0.443 & 0. & 5.08 & 0.078 & 0 . & 146 \\
\hline & 3 & 1 & 0.7 & & 0 & 1.93 & 7.76 & 25.0 & 0.060 & 2.61 & 0. & 8 & 0 & & 8 & 56 & 4 \\
\hline & 3 & 1 & 0.86 & & & & & & & & & & & & & & \\
\hline 88 & 3 & 1 & 0.96 & & 0. & 1.95 & 7.86 & & 0 & 2.69 & 0.39 & 32 & 0 & 4.96 & 0.076 & 9 & 144 \\
\hline DP19389 & 3 & 1 & 1.06 & & 0.35 & 1.94 & 7.86 & 25.2 & 0.067 & 2.63 & 0.39 & 0.435 & 0.66 & 4.98 & 0.078 & 0. & 142 \\
\hline 19390 & 3 & 1 & 1.16 & & $3+3>3$ & 1.96 & 7.85 & 25.3 & 0.069 & 2.63 & 0.39 & 0.440 & 0.68 & 4.98 & 0.078 & 11 & 141 \\
\hline DP19391 & 3 & 1 & 1.26 & & 0.3 & 1.96 & 7.90 & 25.6 & 0.071 & 2.67 & 0.39 & 0.451 & 0. & .01 & 0.079 & 2 & 140 \\
\hline 19392 & 3 & 1 & 1.36 & & 0.35 & 1.98 & 7.87 & 24.9 & 0.068 & 2.64 & 0.36 & 0.432 & 0. & 4 & 0.080 & 48 & 134 \\
\hline 3 & 3 & 1 & $1.4 \mathrm{C}$ & & & 1.95 & 7.89 & 25.4 & 0.073 & 2.60 & 0.35 & 0.433 & 0. & 4.95 & 0.081 & 0. & 142 \\
\hline & 3 & 2 & 0.06 & & & 1.95 & $7.8-8$ & 25.3 & 0.073 & 2.62 & 0. & 0 & & & & & 142 \\
\hline DP19 & 3 & 2 & 0.16 & & 0.31 & 1.93 & 7.78 & 25. & .074 & 2.59 & 0 & 0 . & 0. & 0 & & & 1 \\
\hline & 3 & 2 & 0.26 & & & 1.97 & 7.93 & 25.4 & 0.071 & 2.69 & 0.40 & 0.444 & 0. & & 0. & & 141 \\
\hline & 3 & 2 & 0.36 & & 0.38 & 1.98 & 7.92 & 25.4 & 0.071 & 2.66 & 0.39 & 0.440 & 0.74 & 4.98 & 0.082 & 54 & 138 \\
\hline & 3 & 2 & 0.46 & & 0.36 & 1.9 & 7.90 & 25.4 & 0.071 & 2.69 & 0.38 & 0.439 & 0 . & & 0.079 & 0.056 & 137 \\
\hline DP19 & 3 & 2 & 0.56 & & & & 1.0 & 25. & 0.073 & 2.71 & 0.40 & 0.444 & 0. & 5.01 & 0.079 & 0.067 & 140 \\
\hline & J & 2 & & & & 2.01 & 9 & 25.8 & 0.072 & 2.73 & 0.38 & 0.448 & 0.76 & 5.03 & 0.080 & 0.057 & 141 \\
\hline
\end{tabular}




\begin{tabular}{|c|c|c|c|c|c|c|c|c|c|c|c|c|c|c|c|c|c|}
\hline Lab ID & $\begin{array}{l}\text { गू } \\
\text { ర }\end{array}$ & 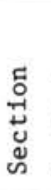 & 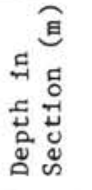 & 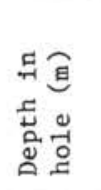 & $\mathrm{Na}$ & $\mathrm{Mg}$ & A1 & S1 & $\mathrm{P}$ & K & $\mathrm{Ca}$ & $\mathrm{Ti}$ & Mn & $\mathrm{Fe}$ & $\mathrm{Ba}$ & S & $\mathrm{H}_{2} \mathrm{O}$ \\
\hline 19401 & 3 & 2 & 0.76 & 76 & 0.42 & 2.06 & 8.03 & 5.6 & 0.073 & .78 & 0.41 & .447 & 0.71 & 5.04 & 0.081 & .085 & 142 \\
\hline DP19402 & 3 & 2 & 0.86 & & 0.34 & 1.97 & 8.00 & & & & 0.33 & & & 4.97 & & & 142 \\
\hline DP19403 & 3 & 2 & 0.96 & 22.96 & 0.35 & 1.96 & 7.87 & 5.1 & 0.066 & .68 & 0.35 & & & & 0.078 & & 142 \\
\hline DP19404 & 3 & 2 & 1.06 & 23.06 & 0.39 & 1.97 & 7.90 & & & & 0.35 & & & & 0.080 & .061 & 140 \\
\hline DP19405 & 3 & 2 & 1.16 & 23.16 & 0.37 & 1.99 & 7.99 & & & 2.72 & & & & 4.97 & .080 & .060 & 137 \\
\hline DP19406 & 3 & 2 & 1.26 & 23.26 & 0.38 & 1.99 & 7.92 & 25.5 & 0.067 & 2.76 & & 0.444 & 0.68 & 5.02 & & 3 & 132 \\
\hline DP19407 & 3 & 2 & 1.36 & 23.36 & 0.40 & 1.98 & 7.85 & 25.4 & 67 & 2. & & 0 & 0.65 & 4.99 & 9 & & 127 \\
\hline DP19408 & 3 & 2 & 1.46 & 23.46 & 0.39 & 1.97 & 7.86 & 25.5 & 0.0 & & 5 & 0 & & & & & 128 \\
\hline DP & 3 & 3 & 0.06 & 23. & 0.41 & 1.96 & 7.87 & 25.6 & 0.0 & & 5 & & & & & 46 & 121 \\
\hline DP19410 & 3 & 3 & 0.16 & 6 & 0.40 & 1.96 & 7.88 & 25.5 & 0.067 & 2.81 & 0.34 & 0. & .62 & 5.02 & 0 & .047 & 128 \\
\hline DP19411 & 3 & 3 & 0.26 & & 0.40 & 1.98 & 7.83 & 25.5 & 0.067 & 2.83 & 0.37 & 0.442 & 0.62 & 5.00 & & & 124 \\
\hline DP19412 & 3 & 3 & 0.36 & & 0.45 & 1.99 & 7.75 & 25.2 & 0.068 & 2.81 & 0.37 & .435 & .62 & 5.00 & & & 118 \\
\hline DP19413 & 3 & 3 & 0.46 & 96 & 0.47 & 2.15 & 7.30 & 24.7 & 0.153 & .78 & 0.61 & 0.503 & & & & 0.082 & 131 \\
\hline DP19414 & 3 & 3 & 0.56 & & 0.40 & 2.1 & 7.2 & 24.9 & & 2.76 & 0.66 & 0.527 & & & 7 & 0.070 & \\
\hline DP1 & 3 & 3 & 0.66 & & 0.38 & 2. & 7.2 & 24.9 & 2 & 2.77 & 0 & 0.5 & 3 & 5. & 20 & 7 & \\
\hline DP1 & 3 & 3 & 0.96 & & 0.48 & 2. & 7.2 & & 0 & 5 & & 0 & & 5. & & & \\
\hline DP1 & 3 & 3 & 1.16 & & & 1 & & & & & & & & & & 3 & \\
\hline DP19418 & 3 & 3 & 1.41 & & 0. & 1 & & 25 & 0 . & 2. & 0.36 & 4 & 2 & 5 . & 7 & & \\
\hline DP19422 & 3 & 4 & 0.26 & & 0.51 & 1.97 & 7.90 & 25.2 & 0.065 & 2.90 & 0.38 & 0.1 & 0.65 & 5.01 & 0.0 & 1 & \\
\hline DP19423 & 3 & 4 & 0.81 & & 0.48 & 1.97 & 7.90 & 25.2 & 0.067 & 2.87 & 0.3 & 8 & 0.66 & 5.01 & 8 & & \\
\hline DP19424 & 3 & 4 & 1.46 & & 0.42 & 2.01 & 7.98 & 25.1 & 0.069 & 2.79 & 0.3 & 7 & 0. & 5.07 & 1 & 3 & \\
\hline DP19419 & 3 & 5 & 0.26 & & 42 & 2.0 & 7.91 & 25.0 & & & $0=$ & & & & & & \\
\hline DP19420 & 3 & 5 & 0.76 & & 0 & 2.8 & 7.9 & 24 & 0. & 8 & & & & 5.07 & 7 & .075 & \\
\hline DP & 3 & 5 & 1.26 & & 2 & 2. & 8. & 25 & 0. & 9 & & & & 5. & & & \\
\hline 425 & 3 & 6 & 0.06 & & .43 & .9 & 7.9 & 0 & 0 & b & 0 & 6 & 0.81 & 5. & 6 & 9 & \\
\hline DP19426 & 3 & 6 & 0.26 & 27.92 & .44 & 2.06 & 7.86 & 25.1 & 0.093 & 2.77 & 1.4 & 3 & $=$ & 5.35 & 2 & 3 & 118 \\
\hline+27 & 3 & 6 & 0.46 & 28.12 & .49 & 2.08 & 7.7 & 4.7 & 109 & . & 0.50 & 459 & & 0 & & & 119 \\
\hline 128 & 3 & 6 & 0.66 & 28.32 & 40 & 0 & 78 & & & & & & & 5. & & & 118 \\
\hline DP1 & 3 & 6 & 0.86 & 28.52 & 0.48 & 2.03 & 7.7 & & 0 & 2 & & & & & & & 113 \\
\hline DP19430 & 3 & 6 & 1.06 & 28.72 & 0.51 & 2.07 & 7.8 & 24.9 & 0.1 & 5 & 0. & 5 & 3 & 4 & & & 13 \\
\hline DP & 3 & 6 & 1.26 & 28.92 & 0.50 & 2.03 & 7.77 & 8 & 4 & 4 & & 6 & & 9 & & & 15 \\
\hline DP & 3 & 6 & 1.46 & 29. & 0. & 2. & $7 . ?$ & & & & & & & 8 & & & 119 \\
\hline DP1 & 3 & 7 & 0.06 & 29 & 0. & 2. & 7. & 2 & 0.1 & 2.76 & 3 & 2 & 1 & 5.3 & 5 & 0 & 119 \\
\hline 134 & 3 & 7 & 0.26 & 29 & 0.54 & .0 & 7.7 & 3 & 0.129 & 2.76 & 0 & 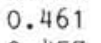 & 2 & .2 & 4 & 2 & 124 \\
\hline 9435 & 3 & 7 & 0.46 & 29.62 & 0.54 & .98 & 7.80 & $4 \cdot 3$ & 0.128 & 2.7 & 0.5 & .457 & 1.2 & 5. & & & 124 \\
\hline 19436 & 4 & 3 & 1.06 & 31.56 & 0.46 & 2.00 & 7.76 & 23.7 & .180 & 2.58 & & & & 5. & & & 151 \\
\hline 37 & 4 & 3 & 1.26 & 31.76 & 0.52 & 9 & 7.69 & 23.7 & & 9 & & & & 5. & & & 139 \\
\hline 38 & 4 & 3 & 1.46 & 31.96 & 0.42 & 1.99 & 7.72 & 23.4 & & 6 & & & & & & & 159 \\
\hline DP & 4 & 4 & 0.06 & & 0.44 & 2. & 7.73 & 23 & & 1 & & & & 1 & 3 & 4 & 49 \\
\hline 440 & 4 & 4 & 0.26 & & 0 . & 18 & 7.7 & & & 2 & & 3 & 6 & 5. & 2 & 8 & 152 \\
\hline DP19441 & 4 & 4 & 0.46 & 32.46 & 0.42 & 1.98 & 7.75 & 23.4 & 5 & 2.65 & 0.69 & 9 & 1.70 & 5.31 & 0. & 0 . & 138 \\
\hline DP19442 & 4 & 4 & 0.66 & 32.66 & 0.43 & 1.97 & 7.79 & 23.7 & 0.172 & 2.77 & 0.58 & 0.430 & 5 & 5.07 & 0. & 0 & 128 \\
\hline DP19443 & 4 & 4 & 0.86 & 32.86 & 0.43 & 2.02 & 7.87 & 24.0 & 0.15 & 2.81 & 0.5 & .4 & .42 & 5. & 1 & & 127 \\
\hline 9444 & 4 & 4 & 1.06 & 33.06 & 0.43 & 1.9 & 7.7 & 23.6 & 0.1 & 2. & & & & 5. & & & 142 \\
\hline DP19445 & 4 & 4 & 1.26 & 33 & 0.44 & 1.98 & 7.72 & 23.5 & 3 & 2.62 & 0 & & & & & & 48 \\
\hline DP1 & 4 & 4 & 1.46 & 33 & 0.42 & 1. & 7.71 & 23.3 & & & & & & & 4 & 3 & 154 \\
\hline & 4 & 5 & 0 & & & & 7.77 & 23.5 & & 2 & & & & 6 & 4 & 6 & 153 \\
\hline 448 & 4 & 5 & 0.26 & & 0 & 1.99 & 7.5 & 2. & & 2 & 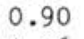 & 2 & 9 & 5.33 & 4 & 3 & 148 \\
\hline DP19449 & 4 & 5 & 0.46 & & 0. & 1.96 & 7.79 & 23.4 & 0. & 2.53 & 0 . & 8 & 2.07 & 5.46 & 0. & 3 & 157 \\
\hline 9450 & 4 & 5 & 0.66 & & 0.4 & 1.99 & 7.54 & 22.9 & 0.28 & 2.57 & 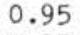 & 0.4 & .03 & 5. & 3 & & 153 \\
\hline 45 & 4 & 5 & 0.86 & & 0.34 & 2.0 & 7.57 & 23.1 & 0.2 & 2.6 & 0.97 & 3 & 5 & 5. & 4 & & 150 \\
\hline 452 & 4 & 5 & 1.06 & 56 & 0.35 & 2.0 & 7.54 & 23.1 & 0.312 & 2.62 & 1. & & & & & & 149 \\
\hline 453 & 4 & 5 & 1.26 & & 3 & 2.0 & 73 & 23.0 & & 2.59 & 1.28 & & & & & & 154 \\
\hline DP19454 & 4 & 5 & 1.46 & & 0.32 & 2.07 & 7.29 & 22.7 & & 2.59 & 1.37 & & & & & 5 & 151 \\
\hline DP19455 & 4 & 6 & 0.06 & & 0.33 & 2.06 & 7.35 & 22.8 & & 2.61 & 1. & & & 5.48 & & & 148 \\
\hline 456 & 4 & 6 & 0.26 & & 0. & 2.07 & 7.30 & 22.7 & & 2.61 & 1. & 0. & 2.12 & 5.47 & 5 & & 149 \\
\hline & 4 & 6 & 0.46 & & 0.34 & 2.01 & 7.11 & 22.2 & 0.446 & 2.55 & 1.3 & 0.430 & 2.00 & 5.35 & 0.074 & 93 & 151 \\
\hline 458 & 4 & 6 & 0.66 & & 0.36 & 2.08 & 7.32 & 22.8 & 0.466 & 2.61 & 1.41 & 0.452 & 1 & & 5 & & 144 \\
\hline 010458 & 4 & 6 & 0.86 & & & 2.08 & & 22.8 & 0.465 & 2.61 & 1.41 & 0.1 & 2.10 & 5.47 & & & 148 \\
\hline DP19460 & 4 & 6 & 1.06 & 36.06 & 0.34 & 2.06 & 7.29 & 22.7 & 0.467 & 2.61 & 1.41 & & 2.07 & & & & 144 \\
\hline DP19461 & 4 & 6 & 1.26 & & 0.32 & 2.06 & $7 \cdot 32$ & 22.8 & 0.446 & 2.61 & & 0.454 & 2.07 & 5.45 & & & 147 \\
\hline & 4 & 6 & 1.46 & & 0.3 & 2.09 & 7.9 & 23.5 & & 2.78 & & 0.4 & & 5. & & & 125 \\
\hline & 4 & 7 & .06 & & & .01 & 7.88 & 23.2 & 0.376 & 2.63 & 1.00 & 样 & 1.00 & 3.72 & 0.073 & 0.081 & 129 \\
\hline
\end{tabular}


Table 4. (Continued).

\begin{tabular}{|c|c|c|c|c|c|c|c|c|c|c|c|c|c|c|c|c|c|}
\hline Lab ID & נّ & 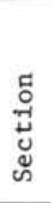 & 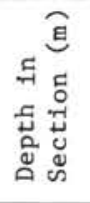 & 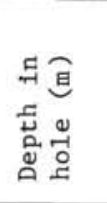 & $\mathrm{Na}$ & $\mathrm{Mg}$ & A1 & $\mathrm{Si}$ & $\mathrm{P}$ & $\mathrm{K}$ & $\mathrm{Ca}$ & T1 & Mn & $\mathrm{Fe}$ & $\mathrm{Ba}$ & S & $\mathrm{H}_{2} \mathrm{O}$ \\
\hline DP19464 & 4 & 7 & 0.26 & & 0.38 & 1.94 & 7.89 & 23.4 & 0.337 & 2.71 & 0.90 & 0.550 & 1.45 & 5.57 & 0.072 & 0.074 & 129 \\
\hline DP19465 & 4 & 7 & 0.46 & 3606 & 0.37 & 1.94 & 7.85 & 23.4 & 0.328 & 2.82 & 0.89 & 0.539 & 1.46 & 5.54 & 0.074 & 0.079 & 129 \\
\hline DP19466 & 5 & 1 & 1.36 & & 0.29 & 2.12 & 8.01 & 23.7 & 0.257 & 2.85 & 0.75 & 0.497 & 1.52 & 5.32 & 0.075 & 0.075 & \\
\hline DP19467 & 5 & 2 & 0.06 & 39.36 & 0.32 & 2.09 & 7.97 & 23.7 & 0.307 & 2.78 & 0.88 & 0.509 & 1.55 & $5 \cdot 32$ & 0.073 & 0.075 & 131 \\
\hline DP19468 & 5 & 2 & 0.26 & 39.56 & 0.34 & 2.03 & 7.98 & 23.6 & 0.350 & 2.66 & 0.99 & 0.542 & 1.61 & 5.46 & 0.071 & 0.079 & 137 \\
\hline DP19469 & 5 & 2 & 0.46 & 39.76 & 0.35 & 1.99 & 7.96 & 23.6 & 0.343 & 2.65 & 0.96 & 0.552 & 1.52 & 5.53 & 0.070 & .076 & 135 \\
\hline DP19470 & 5 & 2 & 0.67 & 39.97 & 0.33 & 1.95 & 7.92 & 23.6 & 0.300 & 2.76 & 0.86 & 0.551 & 1.43 & 5.55 & 0.071 & .074 & 139 \\
\hline DP19471 & 5 & 2 & 0.87 & 40.17 & 0.32 & 1.93 & 7.91 & 23.6 & 0.295 & 2.82 & 0.85 & 0.540 & 1.44 & 5.51 & 0.070 & 0.071 & 141 \\
\hline DP19472 & 5 & 2 & 1.07 & 40.37 & 0.35 & 1.95 & 7.94 & 23.6 & 0.326 & 2.83 & 0.93 & 0.555 & 1.52 & 5.57 & 0.074 & 0.081 & 137 \\
\hline DP19473 & 5 & 2 & 1.26 & 40.56 & 0.34 & 1.98 & 7.91 & 23.6 & 0.341 & 2.76 & 0.94 & 0.574 & 1.58 & 5.59 & 0.075 & 0.080 & 142 \\
\hline DP19474 & 5 & 2 & 1.46 & 40.76 & 0.32 & 1.99 & 7.89 & 23.4 & 0.303 & 2.65 & 0.88 & 0.590 & 1.61 & 5.63 & 0.073 & 0.072 & 146 \\
\hline DP19475 & 5 & 3 & 0.06 & 40.86 & 0.30 & 2.00 & 7.93 & 23.5 & 0.302 & 2.65 & 0.88 & 0.609 & 1.66 & 5.71 & 0.076 & 0.073 & 147 \\
\hline DP19476 & 5 & 3 & 0.26 & 41.46 & 0.22 & 2.05 & 7.87 & 23.3 & 0.320 & 2.59 & 0.91 & 0.605 & 1.64 & 5.66 & 0.076 & 0.087 & 154 \\
\hline DP19477 & 5 & 3 & 0.46 & 41.66 & 0.30 & 2.10 & 7.79 & 23.3 & 0.284 & 2.80 & 0.81 & 0.673 & 1.50 & 6. & 0.075 & 0.067 & 141 \\
\hline DP19478 & 5 & 3 & 0.66 & 41.86 & 0.35 & 2.06 & 7.57 & 22.7 & 0.313 & 2.72 & 0 & 0.7 & 1.38 & 6 & 0.073 & 3 & 143 \\
\hline DP19479 & 5 & 3 & 0.86 & 42.66 & 0.38 & 2.07 & 7.69 & 23.4 & 0.314 & 3.10 & 0.84 & 0.733 & 1.18 & 6.22 & 0.074 & 0.071 & 130 \\
\hline DP19480 & 5 & 3 & 1.06 & 42.86 & 0.34 & 2.13 & 7.65 & 23.7 & 0.238 & 3.18 & 0.67 & 0.688 & 1.16 & 6.06 & 0.074 & 0.060 & 120 \\
\hline DP19481 & 5 & 3 & 1.26 & 43.06 & 0.30 & 2.17 & 7.80 & 24.1 & 0. & 3.19 & 0.71 & 0.633 & 1.23 & 5.90 & 0.075 & 0.060 & 114 \\
\hline DP19482 & 5 & 3 & 1.46 & 43.26 & 0.27 & 2.22 & 7.66 & 24.0 & 0.255 & 3.13 & 0.69 & 0.561 & 1.18 & 5.63 & 0.075 & 0.055 & 109 \\
\hline DP19483 & 5 & 4 & 0.06 & 43.36 & 0.27 & 2.25 & 7.73 & 24.1 & 0.2 & 3.17 & 0.70 & 0.5 & 1.21 & 5.70 & 0.076 & 0.058 & 108 \\
\hline DP19484 & 5 & 4 & 0.26 & 43.56 & 0.26 & 2.24 & 7.59 & 24.0 & 0.2 & 6 & 0.75 & 2 & 1.20 & 9 & 0.075 & 1 & 114 \\
\hline DP19485 & 5 & 4 & 0.46 & 43.76 & 0.26 & 2.25 & 7.73 & 24.2 & 0. & 8 & 0.77 & 6 & 5 & 5. & 0.076 & 66 & 108 \\
\hline DP19486 & 5 & 4 & 0.66 & 43.96 & 0.28 & 2.26 & 7.67 & 24.0 & 0.248 & 3 & 0 & 0.593 & 1. & 5.83 & 0.076 & 0 . & 109 \\
\hline DP19487 & 5 & 4 & 0.86 & 44.16 & 0.37 & 2.11 & 7.75 & 24.0 & 0.231 & 3.30 & 0.65 & 0.674 & 0.93 & 5.98 & 0.074 & 0.053 & 103 \\
\hline DP19488 & 5 & 4 & 1.06 & 44.36 & 0.29 & 2.26 & 7.79 & 23.9 & 0.240 & 3.04 & 0.67 & 0.616 & 1.09 & 5.95 & 0.073 & 0 & 108 \\
\hline DP19489 & 5 & 4 & 1.26 & 44.56 & 0.28 & 2.27 & 7.82 & 24.0 & 0.2 & 3.03 & 0.70 & 0.642 & 1.05 & 6.10 & 0.073 & 0. & 108 \\
\hline DP19490 & 5 & 4 & 1.46 & 44.76 & 0.29 & 2.29 & 7.67 & 23.7 & 0.2 & 3.02 & 0.78 & 8 & 1.09 & 6.08 & 0.074 & 6 & 163 \\
\hline DP19491 & 5 & 5 & 0.06 & 44.86 & 0.30 & 2.28 & 7.74 & 23.9 & 0.3 & 6 & 0.81 & 8 & 1.13 & 6. & 0.075 & 7 & 111 \\
\hline DP19492 & 5 & 5 & 0.26 & 45.06 & 0.36 & 2.19 & 7.74 & 23.9 & 0 . & 7 & 0. & & 4 & 7 & 4 & & 109 \\
\hline DP19493 & 5 & 5 & 0.46 & 45.26 & 0.29 & 2.27 & 7.80 & 23.7 & 0.298 & 1 & 0.79 & 0. & 1.12 & 6.07 & 0.075 & 70 & 106 \\
\hline DP19494 & 5 & 5 & 0.66 & 45.46 & 0.31 & 2.22 & 7.81 & 23.6 & 0.269 & 2.97 & 0.74 & 0.599 & 1.07 & 6.15 & 0.073 & 0.066 & 108 \\
\hline DP19495 & 5 & 5 & 0.86 & 45.66 & 0.29 & 2.28 & 7.85 & 23.7 & 0.287 & 2.94 & 0.79 & 0.602 & 1.13 & 6.18 & 0.076 & 0.065 & 107 \\
\hline DP19496 & 5 & 5 & 1.07 & 45.87 & 0.32 & 2.22 & 7.83 & 23.5 & 0.307 & 2.90 & 0.79 & 0.615 & 1.11 & 6.32 & 0.074 & 0.0 & 108 \\
\hline DP19497 & 5 & 5 & 1.26 & 46.06 & 0.32 & 2.21 & 7.78 & 23.3 & 0.287 & 2.89 & 0.75 & 30 & 1.14 & 6.47 & 0.074 & 0. & 114 \\
\hline DP19498 & 5 & 5 & 1.46 & 46.26 & 0.35 & 2.16 & 7.77 & 23.6 & 0.267 & 3.06 & 0.73 & 3 & 7 & 6.61 & 73 & 6 & 110 \\
\hline DP19499 & 5 & 6 & 0.06 & 46.36 & 0.38 & 2.12 & 7.74 & 23.5 & 0. & 7 & 0. & 9 & 3 & 7 & 73 & 5 & 110 \\
\hline DP19500 & 5 & 6 & 0.26 & 46.56 & 0.38 & 2.14 & 7.77 & 23.2 & 0. & 2.87 & 0.80 & 0.7 & 1.04 & 7.05 & 0.073 & 0. & 114 \\
\hline DP19501 & 5 & 6 & 0.46 & 46.76 & 0.36 & 2.16 & 7.78 & 23.1 & 0.320 & 2.80 & 0.83 & 0.702 & 1.08 & 7.02 & 0.073 & 0.070 & 115 \\
\hline DP19502 & 5 & 6 & 0.66 & 46.96 & 0.35 & 2.15 & 7.87 & 23.1 & 0.319 & 2.77 & 0.84 & 0.726 & 1.04 & 7.01 & 0.073 & 0.067 & 113 \\
\hline DP19503 & 5 & 6 & 0.86 & 47.16 & 0.32 & 2.18 & 7.80 & 23.2 & 0.350 & 2.83 & 0.91 & 0.698 & 1.09 & 6.70 & 0.072 & 0.068 & 118 \\
\hline DP19504 & 5 & 6 & 1.06 & 47.36 & 0.27 & 2.11 & 7.28 & 22.9 & 0.674 & 3.08 & 1.78 & 0.560 & 1.27 & 5.95 & 0.076 & & 125 \\
\hline DP19505 & 6 & 1 & 1.26 & 49.92 & 0.25 & 2.17 & 7.09 & 22.8 & 0.819 & 3.26 & 2.14 & 10 & 1.53 & 5.22 & 0.074 & & 129 \\
\hline DP19506 & 6 & 1 & 1.46 & 50.12 & 0.26 & 2.18 & 7.13 & 9 & 0.7 & & 2. & 0 . & 1.56 & 5.21 & 77 & 2 & 126 \\
\hline DP19507 & 6 & 2 & 0.06 & 50.22 & 0.18 & 2.19 & 7.19 & 23.1 & 0.7 & 3.30 & 2.02 & 0.426 & 1.55 & 5.25 & 0. & 06 & 150 \\
\hline DP19508 & 6 & 2 & 0.26 & 50.42 & 0.24 & 2.12 & 7.17 & 22.7 & 0.789 & 3.35 & 2.08 & 0.379 & 1.51 & 4.92 & 0.072 & 0.103 & 133 \\
\hline DP19509 & 6 & 2 & 0.46 & 50.62 & 0.25 & 2.14 & 7.37 & 23.2 & 0.725 & 3.36 & 1.93 & 0.407 & 1.37 & 5.06 & 0.077 & 0.090 & 119 \\
\hline DP19510 & 6 & 2 & 0.66 & 50.82 & 0.28 & 2.14 & 7.27 & 22.9 & 0.823 & 3.30 & 2.09 & 0.399 & 1.40 & 5.13 & 0.078 & 0.108 & 117 \\
\hline DP19511 & 6 & 2 & 0.86 & .02 & 0.28 & 2.09 & 7.08 & 22.4 & 0.882 & 3.27 & 2.31 & 0.384 & 1.40 & 5.06 & 0.075 & 0.115 & 115 \\
\hline DP19512 & 6 & 2 & 1.06 & 22 & 0.29 & 2.11 & 7.16 & 22.6 & 0.950 & 3.31 & 2.47 & 0.393 & 1.39 & 5.08 & 0.078 & & 114 \\
\hline 513 & 6 & 2 & 1.26 & .42 & 0.32 & 2.04 & 7.12 & 22.5 & 1.0 & & & & 1.45 & 5.00 & 75 & & 121 \\
\hline DP19514 & 6 & 2 & 1.46 & 1.62 & 0.33 & 2.02 & 7.03 & 22.0 & 1.218 & 3.33 & 2.97 & 0.366 & 1.46 & 4.92 & 0.076 & & 119 \\
\hline DP19515 & 6 & 3 & 0.06 & 51.72 & 0.32 & 2.07 & 7.18 & 22.5 & 0.958 & 3.37 & 2.40 & 0.370 & 1.41 & 5.02 & 0.075 & 0. & 115 \\
\hline DP19516 & 6 & 3 & 0.26 & 51.92 & 0.31 & 2.11 & 7.20 & 22.8 & 0.914 & 3.30 & 2.32 & 0.389 & 1.30 & 5.19 & 0.077 & 0.118 & 116 \\
\hline DP19517 & 6 & 3 & 0.46 & 52.12 & 0.33 & 2.17 & 7.17 & 22.9 & 0.733 & 3.23 & 1.92 & 0.428 & 1.30 & 5.47 & 0.078 & 0.106 & 111 \\
\hline DP19518 & 6 & 3 & 0.67 & 53.13 & 0.33 & 2.20 & 7.48 & 23.7 & 0.569 & 3.42 & 1.51 & 0.417 & 1.20 & 5.53 & 0.076 & 0.095 & 108 \\
\hline DP19519 & 6 & 3 & 0.86 & 3.32 & 0.34 & 2.16 & 7.42 & 23.5 & 0.544 & 3.41 & 1.46 & 0.392 & 1.20 & 5.31 & 0.075 & 0.094 & 110 \\
\hline DP19520 & 6 & 3 & 1.06 & .52 & 0.34 & 2.16 & 7.48 & 23.5 & 0.516 & 3.36 & 1.38 & & 1.24 & 5.46 & 0.074 & & 107 \\
\hline DP19521 & 6 & 3 & 1.26 & .72 & 0.35 & 2.16 & 7.45 & 23.6 & 0.414 & 3.43 & 1.15 & 0.3 & 1.29 & 5.28 & 0.076 & & 112 \\
\hline DP19522 & 6 & 3 & 1.46 & .92 & 0.34 & 2.14 & 7.43 & 23.6 & 0.354 & & 1.02 & & 1.25 & 5.2 & 0.075 & & 96 \\
\hline DP19523 & 6 & 4 & 0.06 & 54.02 & 0.34 & 2.14 & 7.48 & 23.8 & 0.355 & 3.47 & 1.02 & 0.401 & 1.35 & 5.24 & 0.077 & & 121 \\
\hline DP19524 & 6 & 4 & 0.26 & 54.22 & 0.33 & 2.15 & 7.43 & 23.7 & & & 0.99 & 0.400 & 1.40 & 5.24 & 0.076 & & 109 \\
\hline DP19525 & 6 & 4 & 0.46 & 54.92 & 0. & 2.15 & 7.10 & 23.2 & & & & 0.402 & 1.67 & 5.40 & 0.079 & 0.092 & 118 \\
\hline DP19526 & 6 & 4 & 0.66 & 55.12 & 0.29 & 2.21 & 6.81 & 23.0 & 0.424 & 3.46 & 1.29 & 0.415 & 2.05 & 6.06 & 0.087 & 0.090 & 121 \\
\hline
\end{tabular}


G. R. HEATH, R. B. KOVAR, C. LOPEZ, G. L. CAMPI

Table 4. (Continued).

\begin{tabular}{|c|c|c|c|c|c|c|c|c|c|c|c|c|c|c|c|c|c|}
\hline Lab ID & 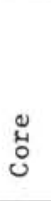 & 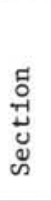 & 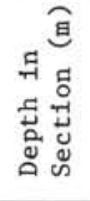 & 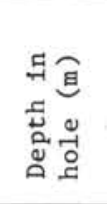 & $\mathrm{Na}$ & $\mathrm{Mg}$ & A1 & Si & P & K & $\mathrm{Ca}$ & $\mathrm{T} 1$ & Mn & $\mathrm{Fe}$ & $\mathrm{Ba}$ & $\mathrm{S}$ & $\mathrm{H}_{2} \mathrm{O}$ \\
\hline DP19527 & 6 & 4 & 0.86 & 32 & 0.31 & .18 & 6.76 & 22.8 & 578 & 1 & 2 & 9 & 05 & 5.63 & 0.087 & 0.093 & 114 \\
\hline DP19528 & 6 & 4 & 1.06 & & .31 & 21 & 6.74 & 22.5 & 74 & 3.49 & 2.53 & 425 & .93 & & .084 & 0.106 & 107 \\
\hline DP19529 & 6 & 4 & 1.46 & 55.92 & 0.29 & 2.29 & 6.74 & 22.9 & 0.426 & 3.41 & 1.56 & 0.387 & 1.91 & 6.25 & 0.084 & 0.088 & 115 \\
\hline DP19530 & 6 & 5 & 0.55 & 56.51 & 0.29 & 2.30 & 6.86 & 23.2 & 0.348 & 3.36 & 0.99 & 0.374 & 1.86 & 6.61 & 0.086 & 0.085 & 126 \\
\hline DP19531 & 6 & 5 & 0.86 & 56.82 & 0.31 & 2.18 & 6.94 & 23.2 & 0.398 & 3.43 & 1.60 & 0.379 & 1.46 & 5.62 & 0.075 & 0.086 & 113 \\
\hline DP19532 & 6 & 5 & 1.06 & 57.02 & 0.30 & 2.17 & 7.00 & 23.4 & 0.324 & 3.36 & 1.14 & 0.379 & 1.43 & 5.85 & 0.078 & 0.074 & 118 \\
\hline DP19533 & 6 & 5 & 1.26 & 57.22 & 0.29 & 2.32 & 6.78 & 23.3 & 0.384 & 3.23 & 1.27 & 0.376 & 1.58 & 6.63 & 0.087 & 0.078 & 119 \\
\hline DP19534 & 6 & 5 & 1.44 & 57.40 & 0.17 & 1.19 & 2.68 & 9.2 & 0.166 & 1.23 & 24.64 & 0.187 & 0.82 & 3.99 & 0.076 & 0.041 & 70 \\
\hline DP19535 & 6 & 6 & 0.47 & 57.93 & 0.27 & 2.39 & 6.74 & 23.0 & 0.291 & 3.20 & 0.86 & 0.356 & 1.74 & 7.84 & 0.091 & 0.076 & 125 \\
\hline DP19536 & 6 & 6 & 0.76 & 58.22 & 0.24 & 2.31 & 6.87 & 23.5 & 0.393 & 3.35 & 1.12 & 0.381 & 1.69 & 6.45 & 0.086 & 0.077 & 121 \\
\hline DP19537 & 6 & 6 & 1.36 & 58.82 & 0.26 & 2.33 & 6.90 & 23.7 & 0.374 & 3.36 & 1.00 & 0.397 & 1.27 & 5.83 & 0.079 & 0.076 & 115 \\
\hline DP19538 & 7 & 3 & 1.45 & 62.45 & 0.27 & 2.47 & 6.97 & 24.4 & 0.364 & 3.38 & 0.94 & 0.410 & 1.23 & 5.46 & 0.118 & 0.087 & 109 \\
\hline DP19539 & 7 & 4 & 0.91 & 63.41 & 0.17 & 2.51 & 6.82 & 24.3 & 0.303 & 3.41 & 0.81 & 0.376 & 1.23 & 5.05 & 0.084 & 0.059 & 121 \\
\hline DP19540 & 7 & 4 & 1.28 & 63 & 0.22 & 2.42 & 6.91 & 24.1 & 0.451 & 3.50 & 1.28 & 0.383 & 1.30 & 5. & 0.085 & 0.065 & 111 \\
\hline DP19541 & 7 & 5 & 0.79 & 79 & 0.21 & 2.49 & 6.77 & 24.6 & 0.378 & 3.41 & 1.02 & 0.395 & 1.24 & 5.08 & 0.089 & 0.066 & 110 \\
\hline 542 & 7 & 6 & 0.26 & 76 & 0.20 & 2.32 & 6.26 & 22.4 & 0.267 & & 4.07 & 0.376 & 1.05 & 4.81 & 0.082 & 0.059 & 112 \\
\hline DP19543 & 7 & 6 & 0.46 & & 0.07 & 2.84 & 6.50 & 24.8 & 0.348 & & 0.82 & 0.388 & 0.95 & 4.89 & 0.088 & 0.056 & 138 \\
\hline DP19544 & 7 & 6 & 0.89 & 66.39 & 0.06 & 2.86 & 6.61 & 25.3 & 0.305 & 3.18 & 0.72 & 0.400 & 0.76 & 4.75 & 0.079 & 0.055 & 133 \\
\hline DP19545 & 7 & 7 & 0.30 & & 0.06 & 2.92 & 6.52 & 24.9 & 0.426 & 3.05 & 0.99 & 0.382 & 0.93 & 4.75 & 0.088 & 0.051 & 139 \\
\hline DP19546 & 8 & 2 & 0.81 & & 0.27 & 2.39 & 6.51 & 24.0 & 0.358 & 3.52 & 2.09 & 0.385 & 1.00 & 4.82 & 0.090 & 0.052 & 102 \\
\hline DP19547 & 8 & 3 & 0.29 & & 0.12 & 2.76 & 6.61 & 25.1 & 0.286 & 3.22 & 0.70 & 0.393 & 0.99 & 4.86 & 0.096 & 0.044 & 126 \\
\hline DP19548 & 8 & 3 & 0.65 & 71.10 & 0.16 & 2.61 & 6.60 & 24.8 & 0.507 & 3.37 & 1.17 & 0.399 & 1.03 & 4.88 & 0.093 & 0.070 & 117 \\
\hline DP19549 & 8 & 3 & 0.96 & 71.41 & 0.19 & 2.57 & 6.18 & 23.5 & 0.345 & & 2.66 & & 0.96 & 4.88 & 0.087 & 0.057 & 116 \\
\hline DP19550 & 8 & 4 & 0.21 & 72.16 & 0.25 & 2.65 & 6.52 & 24.7 & 0.466 & 3.27 & 1.20 & 0.388 & 1.23 & 5.95 & 0.087 & 0.059 & 108 \\
\hline DP19551 & 8 & 4 & 0.41 & 72.36 & 0.38 & 2.50 & 6.24 & 23.7 & 0.479 & 3.36 & 1.33 & 0.347 & 1.59 & 6.22 & 0.094 & 0.075 & 103 \\
\hline DP19552 & 8 & 4 & 0.99 & 72.94 & 0.37 & 2.46 & 6.00 & 23.4 & 0.575 & 3.25 & 2.41 & 0.363 & 1.32 & 6.01 & 0.093 & 0.070 & 100 \\
\hline DP19553 & 8 & 5 & 0.58 & 74.03 & 0.24 & 2.73 & 6.36 & 25.6 & 0.337 & 3.39 & 0.84 & 0.400 & 0.77 & 5.52 & 0.083 & 0.057 & 109 \\
\hline DP19554 & 8 & 5 & 1.42 & 74.87 & 0.20 & 2.98 & 6.50 & 25.5 & 0.302 & 3.19 & 0.78 & 0.405 & 0.85 & 5.38 & 0.084 & 0.056 & 113 \\
\hline DP19555 & 8 & 6 & 0.44 & 75.39 & 0.19 & 3.03 & 6.33 & 25.3 & 0.266 & 3.16 & 1.10 & 0.397 & 0.82 & 5.18 & 0.086 & 0.052 & 112 \\
\hline
\end{tabular}

Note: Depth in hole omitted for disturbed samples. 
Table 5. Elemental abundance (wt.\%) and water content (\% dry wt.) of samples making up the composite section of Site 576.

\begin{tabular}{|c|c|c|c|c|c|c|c|c|c|c|c|c|c|c|c|c|c|}
\hline 뭄 & $\stackrel{\stackrel{H}{\circ}}{0}$ & 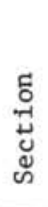 & 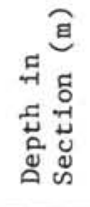 & 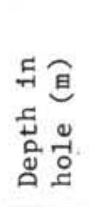 & $\mathrm{Na}$ & $\mathrm{Mg}$ & 1 & 31 & $\mathrm{P}$ & K & $\mathrm{Ca}$ & Ti & Mn & $\mathrm{Fe}$ & $\mathrm{Ba}$ & $\mathrm{S}$ & $\mathrm{H}_{2} \mathrm{O}$ \\
\hline $6 \mathrm{~B}$ & 1 & 1 & 06 & 6 & & 5 & & & 19 & 51 & 40 & 07 & 24 & .66 & 95 & 31 & $y$ \\
\hline 105 & 1 & 1 & & & & & & & & & & & & & & & \\
\hline $6 \mathrm{~B}$ & 1 & 1 & & & & & & & & & & & & & & & \\
\hline $76 \mathrm{~B}$ & 1 & 1 & 0 & & & & & & & & & & & & & & \\
\hline 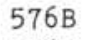 & 1 & 1 & & & & & & & & & & & & & & & \\
\hline $76 \mathrm{~B}$ & 1 & 1 & 0 & & & & & & & & & & & & & & \\
\hline $76 \mathrm{~B}$ & 1 & 1 & 0.66 & 0 & & & & & & & & & & & & & 32 \\
\hline $76 \mathrm{~B}$ & 1 & 1 & 0.76 & 6 & & & & & & & & & & & & & \\
\hline $6 \mathrm{~B}$ & 1 & 1 & 0.86 & .86 & .43 & 1.90 & 4 & & & & & & & & & & 137 \\
\hline $6 \mathrm{~B}$ & 1 & 1 & 0.96 & 96 & .46 & 1.85 & .6 & & & & 6 & - & & 7 & & & 137 \\
\hline $76 \mathrm{~B}$ & 1 & 1 & 1.06 & 1.06 & .43 & .85 & 7. & 0.3 & • & & .48 & 1 & & 7 & & & 50 \\
\hline $6 \mathrm{~B}$ & 1 & 1 & 1.16 & 1.16 & 0.43 & .84 & $*$ & 4 & 3 & 7 & 8 & 5 & & & & & \\
\hline & 1 & 1 & 1.26 & 1.26 & .39 & 1.82 & 1. & & 0.054 & 2.54 & .44 & 0. & .58 & .75 & & & 46 \\
\hline 78 & 1 & 1 & 1.36 & 1.36 & 0.42 & 1.87 & 10 & & 0.055 & .55 & .44 & & & & & & \\
\hline 6.0 & 1 & 1 & 1.46 & 1.46 & 0.44 & & 7.1 & & 0.055 & .55 & .47 & 0. & & & & & 36 \\
\hline & 1 & 2 & 0.06 & 6 & & & & & 0.053 & 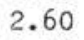 & .43 & & & & & & \\
\hline $76 \mathrm{~B}$ & 1 & 2 & .16 & & & & & & & & & & & & & & 55 \\
\hline $76 \mathrm{~B}$ & 1 & 2 & 0.26 & & & & & & & & & & & & & & \\
\hline 76 & 1 & 2 & .36 & & & & & & & & & & & & & & 9 \\
\hline 6 & 1 & 2 & .46 & 96 & & & & & & & & & & & & & \\
\hline $76 \mathrm{~B}$ & 1 & 2 & .56 & 06 & & & & & & & & & & & & & 17 \\
\hline 16 & 1 & 2 & .66 & & & & & & & & & & & & & & \\
\hline & 1 & 2 & .76 & & & & & & & & & & & & & & 14 \\
\hline 76 & 1 & 2 & 0 & & & & & & & & & & & & & & \\
\hline 76 & 1 & 2 & & & & & & & & & & & & & & & \\
\hline 76 & 1 & 2 & 1 & & & & & & & & & & & & & & 0 \\
\hline 76 & 1 & 2 & 1 & & & & & & & & & & & & & & 201 \\
\hline $76 \mathrm{~B}$ & 1 & 2 & 1 & & & & & & & & & & & & & & 188 \\
\hline $76 \mathrm{E}$ & 1 & 2 & 1 & & & & & & & & & & & & & & 181 \\
\hline 76 & 1 & 2 & 1 & 2 & & & & & & & & & & & & & 252 \\
\hline $76 \mathrm{~B}$ & 1 & 3 & 0.06 & 3 & & & & & & & & & & & & & 274 \\
\hline 61 & 1 & 3 & 0.16 & 3 & 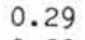 & 1 & & & & & & & & & & & 189 \\
\hline & 1 & 3 & 0.26 & 3 & . 30 & 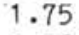 & 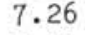 & 6 & 33 & 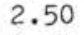 & 5 & & & & & & 185 \\
\hline 6 & 1 & 3 & 0.36 & 3 & .36 & . & & & & 3 & 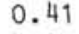 & & & & & & 66 \\
\hline & 1 & 3 & 0.47 & 3 & $\cdot 3$ & 1. & & & 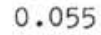 & & 3 & & & & & & 165 \\
\hline $6 \mathrm{~F}$ & 1 & 3 & 0.56 & 3 & 3 & 1.7 & & & & & 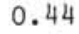 & & & & & & 53 \\
\hline 6 & 1 & 3 & 0.66 & -7 & .4 & $\therefore$ & & & 0 & 2 & 2 & & & & & & 50 \\
\hline & 1 & 3 & 0 & & 0 & 8 & & & & & & & & & & & 11 \\
\hline & 1 & 3 & 86 & & & & & & & 2 & 0 & & & & & & 52 \\
\hline & 1 & 3 & & & & & & & & & & & & & & & 7 \\
\hline & 1 & 3 & & & & & & & & & & & & & & & \\
\hline & 1 & 3 & & & & & & & & & & & & & & & \\
\hline & 1 & 3 & & & & & & & & & & & & & & & \\
\hline & 1 & 3 & & & & & & & & & & & & & & & 144 \\
\hline & 1 & 3 & 1 & & & & & & & & & & & & & & 151 \\
\hline $76 \mathrm{~B}$ & 1 & 4 & 6 & & & & & & & & & & & & & & 144 \\
\hline & 1 & 4 & & & & & & & & & & & & & & & 140 \\
\hline $6 \mathrm{~B}$ & 1 & 4 & & & & & & & & & .44 & & & & & & 148 \\
\hline & 1 & 4 & & & & & & & & & & & & & & & \\
\hline & 1 & 4 & & & & & & & & & & & & & & & 144 \\
\hline & 1 & 4 & 0.56 & & & & & & & & .42 & & & & & & 145 \\
\hline $6 \mathrm{~B}$ & 1 & 4 & & & & & & & & & & & & & & & 152 \\
\hline & 1 & 4 & & & & & & & & & & & & & & & 151 \\
\hline & 1 & 4 & & & & & & & & & & & & & & & \\
\hline & 1 & 4 & & & & & & & & & & & & & & & 153 \\
\hline & 1 & 4 & & & & & & & & & & & & & & & \\
\hline & 1 & 4 & & & & & & & 0.052 & & 2 & & & & 0. & 7 & 142 \\
\hline & 1 & 4 & & & & & & & & & 0 & & & & 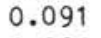 & 7 & 139 \\
\hline & 1 & 4 & & & & & & & & & & & & & & & 138 \\
\hline $76 \mathrm{E}$ & 1 & 4 & & & & & & & & & & & 0.28 & 4.84 & 0.092 & 0.055 & 136 \\
\hline & 1 & 2 & & & & & & & & & 0.46 & 0.433 & 0.34 & 4.90 & 0.091 & 0.050 & 139 \\
\hline
\end{tabular}


Table 5. (Continued).

\begin{tabular}{|c|c|c|c|c|c|c|c|c|c|c|c|c|c|c|c|c|c|}
\hline $\begin{array}{l}\stackrel{0}{-1} \\
\text { 오 }\end{array}$ & 峁 & 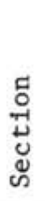 & 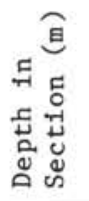 & 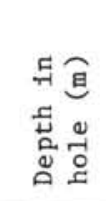 & $\mathrm{Na}$ & $\mathrm{Mg}$ & $\mathrm{A} 1$ & Si & $\mathrm{P}$ & K & $\mathrm{Ca}$ & Ti & Mn & $\mathrm{Fe}$ & $\mathrm{Ba}$ & $\mathrm{S}$ & $\mathrm{H}_{2} \mathrm{O}$ \\
\hline $576 \mathrm{~B}$ & 1 & 5 & 0.16 & 6.16 & 0.39 & 1.88 & 7.59 & 26.0 & 0.055 & 2.60 & 0.47 & 0.422 & 0.35 & 4.79 & 0.092 & 0.051 & 143 \\
\hline $576 \mathrm{~B}$ & 1 & 5 & 0.26 & 6.26 & 0.36 & 1.90 & 7.67 & 26.2 & 0.054 & 2.63 & 0.44 & 0.422 & 0.32 & 4.82 & 0.093 & 0.047 & 147 \\
\hline $576 \mathrm{~B}$ & 1 & 5 & 0.36 & 6.36 & 0.32 & 1.88 & 7.62 & 26.4 & 0.055 & 2.61 & 0.47 & 0.419 & 0.35 & 4.76 & 0.091 & 0.048 & 154 \\
\hline $576 \mathrm{~B}$ & 1 & 5 & 0.46 & 6.46 & 0.41 & 1.84 & 7.53 & 26.2 & 0.056 & 2.59 & 0.49 & 0.414 & 0.35 & 4.74 & 0.093 & 0.051 & 138 \\
\hline $576 \mathrm{~B}$ & 1 & 5 & 0.56 & 6.56 & 0.46 & 1.86 & 7.56 & 26.5 & 0.056 & 2.62 & 0.51 & 0.419 & 0.34 & 4.75 & 0.094 & 0.060 & 133 \\
\hline $576 \mathrm{~B}$ & 1 & 5 & 0.66 & 6.66 & 0.39 & 1.90 & 7.60 & 26.4 & 0.054 & 2.66 & 0.45 & 0.428 & 0.30 & 4.80 & 0.097 & 0.054 & 139 \\
\hline $576 \mathrm{~B}$ & 1 & 5 & 0.76 & 6.76 & 0.41 & 1.91 & 7.58 & 26.4 & 0.054 & 2.65 & 0.46 & 0.427 & 0.31 & 4.79 & 0.096 & 0.054 & 137 \\
\hline $576 \mathrm{~B}$ & 1 & 5 & 0.86 & 6.86 & 0.38 & 1.95 & 7.50 & 26.3 & 0.054 & 2.60 & 0.45 & 0.414 & 0.38 & 4.77 & 0.095 & 0.046 & 152 \\
\hline $576 \mathrm{~B}$ & 1 & 5 & 0.96 & 6.96 & 0.38 & 1.89 & 7.43 & 26.6 & 0.048 & 2.58 & 0.45 & 0.407 & 0.36 & 4.58 & 0.083 & 0.032 & 147 \\
\hline 576 & 2 & 1 & 0.06 & 7.01 & 0.53 & 2.01 & 7.61 & 26.1 & 0.051 & 2.69 & 0.45 & 0.425 & 0.63 & 4.79 & 0.091 & 0.060 & 134 \\
\hline 576 & 2 & 1 & 0.16 & 7.11 & 0.55 & 2.03 & 7.60 & 26.4 & 0.047 & 2.73 & 0.49 & 0.430 & 0.28 & 4.80 & 0.085 & 0.091 & 149 \\
\hline 576 & 2 & 1 & 0.26 & 7.21 & 0.52 & 1.97 & 7.59 & 26.4 & 0.050 & 2.66 & 0.44 & 0.417 & 0.36 & 4.70 & 0.088 & 0.050 & 159 \\
\hline 576 & 2 & 1 & 0.36 & 7.31 & 0.59 & 1.98 & 7.48 & 26.2 & 0.050 & 2.67 & 0.51 & 0.417 & & 4.68 & 0.096 & & 145 \\
\hline 576 & 2 & 1 & 0.46 & 7.41 & 0.51 & 2.01 & 7.56 & 26.4 & 0.053 & 2.69 & 0.50 & 0.417 & 0.38 & 4.70 & 0. & & 158 \\
\hline 576 & 2 & 1 & 0.56 & 7.51 & 0.57 & 2.04 & 7.59 & 26.3 & 0.052 & 2.75 & 0.48 & 0.431 & 0.33 & & 2 & & 144 \\
\hline 576 & 2 & 1 & 0.66 & 7.61 & 0.48 & 2.03 & 7.63 & 26.1 & 0.051 & 2.77 & 0.43 & 0.428 & & 82 & 0. & 76 & 143 \\
\hline 576 & 2 & 1 & 0.76 & 7.71 & 0.56 & 2.08 & 7.61 & 26.3 & 0.053 & 2.77 & 0.48 & 0.431 & 7 & 83 & 1 & 95 & 146 \\
\hline 576 & 2 & 1 & 0.86 & 7.81 & 0.52 & 2.01 & 7.67 & 26.3 & 0.050 & 2.72 & 0.41 & 0.430 & 0.25 & 4.86 & 0.086 & 52 & 144 \\
\hline 576 & 2 & 1 & 0.96 & 7.91 & 0.46 & 2.01 & 7.53 & 26.1 & 0.051 & 2.68 & 0.42 & 0.418 & 0.29 & 4.76 & 0. & & 159 \\
\hline 576 & 2 & 1 & 1.06 & 8.01 & 0.44 & 2.04 & 7.58 & 26.1 & 0.050 & 2.70 & 0.42 & 0.414 & 0.31 & 4.75 & 0.095 & 0.067 & 159 \\
\hline 576 & 2 & 1 & 1.16 & 8.11 & 0.59 & 2.07 & 7.64 & 26.3 & 0.051 & 2.78 & 0.50 & 0.430 & 0.32 & 4.87 & 0.095 & 0.095 & 146 \\
\hline 576 & 2 & 1 & 1.26 & 8.21 & 0.43 & 2.05 & 7.62 & 26.2 & 0.053 & 2.67 & 0.44 & 0. & 0.38 & 4.77 & 0.089 & 0.065 & 168 \\
\hline 576 & 2 & 1 & 1.36 & 8.31 & 0.39 & 2.01 & 7.49 & 26.4 & 0.052 & 2.61 & 0.46 & 0.408 & 0.39 & 4.65 & 0.088 & 0.073 & 178 \\
\hline 576 & 2 & 1 & 1.46 & 8.41 & 0.48 & 1.98 & 7.58 & 26.2 & 0.057 & 2.59 & 0.42 & 0.413 & 0.39 & 4.71 & 0.088 & 0.036 & 154 \\
\hline 576 & 2 & 2 & 0.06 & 8.51 & 0.49 & 2.00 & 7.61 & 26.3 & 0.055 & 2.65 & 0.45 & 0.418 & 0.34 & 4.77 & 0.089 & 0.049 & 145 \\
\hline 576 & 2 & 2 & 0.16 & 8.61 & 0.46 & 1.99 & 7.58 & 26.2 & 0.054 & 2.61 & 0.47 & 0.418 & 0.39 & 4.79 & 0. & 0.049 & 147 \\
\hline 576 & 2 & 2 & 0.26 & 8.71 & 0.45 & 1.96 & 7.59 & 26.4 & 0.053 & 2.58 & 0.46 & 0.414 & 0.39 & 4.77 & 0.087 & 0.032 & 159 \\
\hline 576 & 2 & 2 & 0.36 & 8.81 & 0.47 & 2.01 & 7.58 & 26.2 & 0.055 & 2.61 & 0.43 & 0.416 & 0.41 & 4.75 & 0. & 48 & 156 \\
\hline 576 & 2 & 2 & 0.46 & 8.91 & 0.46 & 1.99 & 7.60 & 26.5 & 0.054 & 2.60 & 0.41 & 0.413 & 0.42 & 4.74 & 0.096 & 0.029 & 161 \\
\hline 576 & 2 & 2 & 0.56 & 9.01 & 0.46 & 2.00 & 7.54 & 26.3 & 0.053 & 2.61 & 0.40 & 0.414 & 0.29 & 4.74 & 0. & 41 & 165 \\
\hline 576 & 2 & 2 & 0.66 & 9.11 & 0.46 & 2.06 & 7.54 & 26.3 & 0.048 & 2.68 & 0.47 & 0.418 & 0.39 & 4.78 & 0 & 72 & 161 \\
\hline 576 & 2 & 2 & 0.76 & 9.21 & 0.45 & 1.98 & 7.48 & 26.3 & 0.049 & 2.59 & 0.42 & 0.410 & 0.39 & 4.72 & 0.092 & 43 & 170 \\
\hline 576 & 2 & 2 & 0.86 & 9.31 & 0.32 & 2.03 & $7 \cdot 36$ & 26.7 & 0.050 & 2.58 & 0.42 & 0.398 & 0.26 & 4.73 & 0.087 & 70 & 207 \\
\hline 576 & 2 & 2 & 0.96 & 9.41 & 0.37 & 1.96 & 7.48 & 26.5 & 0.056 & 2.55 & 0.43 & 0.411 & 0.26 & 4.68 & 0.087 & 43 & 194 \\
\hline 576 & 2 & 2 & 1.06 & 9.51 & 0.49 & 2.02 & 7.51 & 26.4 & 0.058 & 2.64 & 0.55 & 0.422 & 0.46 & 4.72 & 35 & 82 & 163 \\
\hline 576 & 2 & 2 & 1.16 & 9.61 & 0.47 & 1.96 & 7.46 & 26.2 & 0.056 & 2.55 & 0.44 & 06 & 0.40 & 4.65 & 85 & 1 & 170 \\
\hline 576 & 2 & 2 & 1.26 & 9.71 & 0.53 & 2.03 & 7.46 & 26.2 & 0.053 & 2.67 & 0.54 & 18 & 36 & 75 & 3 & 31 & 149 \\
\hline 576 & 2 & 2 & 1.36 & 9.81 & 0.48 & 1.97 & 7.46 & 26.2 & 0.054 & 2.61 & 0.46 & 12 & 37 & 71 & 4 & 0.055 & 154 \\
\hline 576 & 2 & 2 & 1.46 & 9.91 & 0. & 2.04 & 7.53 & 26 & 0.0 & 2.76 & 0 . & & 39 & 4.83 & 0.098 & 0.107 & 154 \\
\hline 576 & 2 & 3 & 0.06 & 0.01 & 0.50 & 2.03 & 7.56 & 26 & 0 & 2.69 & 0.41 & 0. & 0.39 & 4.75 & 0. & 0.088 & 176 \\
\hline 576 & 2 & 3 & 0.16 & 11 & 0.5 & 2.05 & 7.44 & 26 & 0.0 & 2.72 & 0.47 & 0.1 & 0.38 & 4.70 & 0.101 & 0.118 & 185 \\
\hline 576 & 2 & 3 & 0.26 & .21 & 0.40 & 1.98 & 7.40 & 26.4 & 0.051 & 2.55 & 0.36 & 0.3 & 0.30 & 4.55 & 0.095 & 0.051 & 218 \\
\hline 576 & 2 & 3 & 0.36 & 31 & 0.29 & 1.83 & 6.98 & 27 & 0.052 & 2.43 & 0.39 & 0 & 0.52 & 4.40 & 0.095 & 0.056 & 218 \\
\hline 576 & 2 & 3 & .46 & & 0.5 & 1.90 & 7.44 & 26.4 & 0.057 & 2.52 & & & 0.49 & 4.64 & 0.086 & 0.033 & 180 \\
\hline 576 & 2 & 3 & 0.56 & 10.51 & 0.5 & 1.95 & 7.3 & 26.3 & 0.053 & 2.59 & & 0. & 0.44 & 4.62 & 0.089 & 0.074 & 163 \\
\hline 576 & 2 & 3 & 0.66 & .61 & 0. & 1.94 & 7.41 & & & 2.60 & 0.50 & 0.408 & 0.41 & 4.67 & 0.087 & 0.059 & 142 \\
\hline 576 & 2 & 3 & 0.76 & 10.71 & 0.60 & 1.91 & 7.31 & 26 & 0.048 & 2.63 & 0.57 & 0.402 & 0.37 & 4.48 & 0.087 & 0.073 & 138 \\
\hline 576 & 2 & 3 & 0.86 & 10.81 & 0.59 & 1.78 & 7.15 & 26.6 & 0.046 & 2.55 & 0.57 & 0.373 & 0.35 & 4.13 & 0.089 & 0.042 & 122 \\
\hline 576 & 2 & 3 & 0.96 & 10.91 & 0.61 & 1.77 & 7.05 & 26.6 & 0.043 & 2.67 & 0.54 & 0.358 & 0.38 & 3.95 & 0.083 & 0.075 & 127 \\
\hline 576 & 2 & 3 & 1.06 & 11.01 & 0.53 & 1.98 & 7.50 & 26.0 & 0.050 & 2.65 & 0.45 & 0.417 & 0.56 & 4.70 & 0.092 & 0.065 & 146 \\
\hline 576 & 2 & 3 & 1.16 & 11.11 & 0.57 & 2.07 & 7.58 & 26.1 & 0.050 & 2.71 & 0.48 & 0.431 & 0.46 & 4.81 & 0.089 & 0.085 & 149 \\
\hline 576 & 2 & 3 & 1.26 & 11.21 & 0.56 & 2.03 & 7.61 & 26.3 & 0.045 & 2.70 & 0.40 & 0.421 & 0.38 & 4.73 & 0.090 & 0.062 & 157 \\
\hline 576 & 2 & 3 & 1.36 & 11.31 & 0.46 & 2.05 & 7.52 & 26.3 & 0.048 & 2.67 & 0.44 & 0.421 & 0.28 & 4.74 & 0.095 & 0.081 & 168 \\
\hline 576 & 2 & 3 & 1.46 & 11.41 & 0.55 & 2.00 & 7.58 & 26.1 & 0.047 & 2.70 & 0.38 & 0.424 & 0.28 & 4.81 & 0.092 & 0.050 & 147 \\
\hline 576 & 2 & 4 & 0.06 & 11.51 & 0.51 & 2.04 & 7.61 & 26.4 & 0.053 & 2.68 & 0.49 & 0.420 & 0.43 & 4.76 & 0.094 & 0.072 & 155 \\
\hline 576 & 2 & 4 & 0.16 & 11.61 & 0.45 & 2.01 & 7.58 & 26.3 & 0.056 & 2.61 & 0.42 & 0.415 & 0.47 & 4.69 & 0.091 & 0.052 & 165 \\
\hline 576 & 2 & 4 & 0.26 & 11.71 & 0.37 & 1.98 & 7.39 & 26.4 & 0.054 & 2.58 & 0.41 & 0.401 & 0.51 & 4.51 & 0.092 & 0.046 & 181 \\
\hline 576 & 2 & 4 & 0.36 & 11.81 & 0.48 & 2.02 & & 26.4 & 0.052 & 2.62 & 0.38 & 0.406 & 0.46 & 4.64 & 0.095 & 0.031 & 164 \\
\hline 576 & 2 & 4 & 0.46 & 11.91 & 0.49 & 1.99 & 7.48 & 26.5 & 0.054 & 2.62 & 0.45 & 0.397 & 0.46 & 4.51 & 0.092 & 0.050 & 149 \\
\hline 576 & 2 & 4 & 0.56 & 12.01 & 0.54 & 1.98 & 7.45 & 26.4 & 0.054 & 2.62 & 0.43 & 0.405 & 0.41 & 4.55 & 0.095 & 0.044 & 140 \\
\hline 576 & 2 & 4 & 0.66 & 12.11 & 0.65 & 1.48 & 6.64 & 27.5 & 0.045 & 3.02 & 0.44 & 0.275 & 0.32 & 3.25 & 0.090 & 0.042 & 119 \\
\hline
\end{tabular}




\begin{tabular}{|c|c|c|c|c|c|c|c|c|c|c|c|c|c|c|c|c|c|}
\hline$\stackrel{0}{\frac{0}{0}}$ & 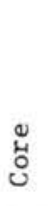 & 范 & 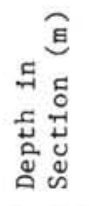 & 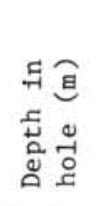 & $\mathrm{Na}$ & $\mathrm{Mg}$ & Al & Si & $\mathrm{P}$ & K & $\mathrm{Ca}$ & Ti & Mn & $\mathrm{Fe}$ & $\mathrm{Ba}$ & S & $\mathrm{H}_{2} \mathrm{O}$ \\
\hline 576 & 2 & 4 & 76 & 21 & 0.49 & 2.00 & 7.60 & 4 & 49 & 2.65 & 0.30 & 0.399 & 37 & 4.54 & .091 & 15 & 178 \\
\hline 576 & 2 & 4 & 86 & 12.31 & 7 & & 6 & & 53 & 1 & & & & & .092 & & 177 \\
\hline 576 & 2 & 4 & 0.96 & 12.41 & 0 & 3 & 7 & & & 2 & & & & & .087 & & 165 \\
\hline 576 & 2 & 4 & 1.06 & 12.51 & 0. & 2 & 7 & & & & & & & & .093 & & 174 \\
\hline 576 & 2 & 4 & 1.16 & 12.61 & 0. & & & & & & & & & & 0.092 & 0. & 180 \\
\hline 576 & 2 & 4 & 1.26 & 12.71 & 0. & & & & & & & & 0.48 & 4.62 & & 0.053 & 175 \\
\hline 576 & 2 & 4 & 1.36 & 12.81 & 0.37 & 2.0 & 7 & 26.2 & . & 2.59 & 0.35 & 0.411 & 0.49 & 4.70 & 0.090 & 0.035 & 176 \\
\hline 576 & 2 & 4 & 1.46 & 12.91 & 0.33 & 2.11 & 9 & 26.4 & 0. & 2.62 & 0.39 & 0.411 & 0.48 & 4.67 & 0.096 & 0.0 & 185 \\
\hline 576 & 2 & 5 & 0.06 & 13.01 & 0.39 & 2.03 & & 26.4 & 0.0 & 2.54 & 0.32 & 0.411 & 0.52 & 4.64 & 0.0 & 0. & 202 \\
\hline 576 & 2 & 5 & 0.16 & 13.11 & 0.32 & 2.06 & 7.50 & 26.4 & 0.0 & 2.55 & 0.39 & 0.2 & 0.51 & 4.56 & 0.0 & & 198 \\
\hline 576 & 2 & 5 & 0.26 & 13.21 & 0.42 & 2.00 & 7.47 & 20.3 & 0.055 & 2.53 & 0.39 & 0.404 & 0.52 & 4.58 & 0.089 & 0.0 & 176 \\
\hline 576 & 2 & 5 & 0.36 & 13.31 & 0.40 & 2.02 & 7.48 & 26.7 & 0.056 & 2.55 & 0.41 & 0.405 & 0.50 & 4.56 & 0.093 & & 183 \\
\hline 576 & 2 & 5 & 0.46 & 13.41 & 0.45 & 2.00 & 7.51 & 26.8 & 0.054 & 2.55 & 0.33 & 0.400 & 0.48 & 4.59 & 0.0 & 0. & 192 \\
\hline 576 & 2 & 5 & 0.56 & 13.51 & 0.35 & 2.06 & 7.50 & 26.4 & 0.053 & 2.62 & 0.36 & 0.400 & 0.42 & 4.58 & & & 181 \\
\hline 76 & 2 & 5 & 0.66 & 13.61 & 0.40 & 2.02 & 7.53 & 26.6 & 0.052 & 2.55 & 0.29 & 0.396 & 0.41 & 4.57 & & & 195 \\
\hline 576 & 2 & 5 & 0.76 & 13.71 & 0.28 & 2.04 & 7.4 & & 0.053 & 2.48 & 0.40 & 0.3 & 0.43 & 4. & & & 220 \\
\hline 576 & 2 & 5 & 0.86 & 13.81 & 0.42 & 1.98 & 7.39 & 26.6 & 0.053 & 2.46 & 0.41 & 0.3 & 0.1 & 4. & & & 192 \\
\hline 576 & 2 & 5 & 0.96 & 13 & 0.44 & 1.99 & 7.4 & & 0.051 & 2.51 & 0.44 & 0.4 & 0. & 4 & & & 171 \\
\hline 576 & 2 & 5 & 1.05 & & 0.46 & 1.99 & 7.38 & 26 & 0.052 & 2.48 & 0.41 & 0. & 0. & 3 & & & 178 \\
\hline $576 \mathrm{~B}$ & 2 & 2 & 0.56 & 14.06 & 0.34 & 1.99 & 7. & & & 2.59 & 0.45 & 0. & 6 & 2 & & & 150 \\
\hline $576 \mathrm{~B}$ & 2 & 2 & & 14.16 & 0.28 & & 7.28 & & & 2.56 & 0.43 & 0. & 5 & 4 & & & 164 \\
\hline $576 \mathrm{~B}$ & 2 & 2 & 0.76 & 14.26 & 0.26 & 2.03 & 7.55 & & & 2.64 & 0.39 & 0. & 7 & 3 & & & 58 \\
\hline $576 \mathrm{~B}$ & 2 & 2 & 0.86 & 4.36 & 0.18 & & 7. & 26 & & 2.59 & 0.34 & 0 . & 8 & 4 & 3 & & 185 \\
\hline $576 \mathrm{~B}$ & 2 & 2 & 0.96 & 4.46 & 0 . & & & & & 2.60 & 0 . & 0. & 0.4 & 3 & & & 8 \\
\hline 576 & 2 & 5 & 6 & 1 & 0 & 2 & 7. & & & 2 & 0. & 2 & 0.38 & 0 & & & 154 \\
\hline 576 & 2 & 5 & 1.26 & 1 & & & & & & 1 & 0 . & & & & & & 172 \\
\hline 576 & 2 & 5 & .36 & 14.71 & 0. & 2. & 7. & & 0 & 2 & 0 & 0 & 3 & 4.63 & 9 & 8 & 172 \\
\hline 576 & 2 & 5 & 1.46 & 14 & 0. & 2. & 7. & & 2 & 8 & 0 & & .45 & 4.65 & 0. & & 178 \\
\hline 576 & 2 & 6 & 0.06 & 14 & 0. & 2. & & & 3 & 3 & 8 & & 0 & 4.64 & 0. & 9 & 172 \\
\hline 576 & 2 & 6 & 0.16 & 15. & 0 & 2. & & & 4 & 2 & 0.37 & 0. & 2 & 4.54 & 0. & & 175 \\
\hline 576 & 2 & 6 & 0.26 & 15.11 & 0.40 & 2 & 0 & 2 & 5 & 2.59 & 0.38 & 0. & 0.41 & 4.44 & 0. & 4 & 178 \\
\hline 576 & 2 & 6 & 0.36 & 15.21 & 0.40 & 2. & 7.57 & 26. & 0.0 & 2.56 & 0.32 & 0.399 & 0.41 & 4.51 & 0.097 & 8 & 197 \\
\hline 576 & 2 & 6 & .46 & 5.31 & 0.41 & 08 & 7.64 & 26.5 & 0.056 & 2.56 & 0.36 & 0.409 & 0.46 & 4.65 & 0.095 & 0 & 172 \\
\hline 576 & 2 & 6 & 0.56 & 15.41 & 0.43 & 2.06 & 7.52 & 26 & 0.057 & 2.44 & 0.33 & 0.404 & 0.52 & 4.55 & 0.0 & 2 & 182 \\
\hline 576 & 2 & 6 & 0.66 & 15.51 & 0.35 & 2.04 & 7.57 & 26. & 0.057 & 2.49 & 0.35 & 0.407 & 0.53 & 4.55 & 0.097 & 1 & 196 \\
\hline 576 & 2 & 6 & 0.76 & 5.61 & 0.34 & 2.07 & 7.5 & 26. & 0.055 & 2.46 & 0.33 & 0.398 & 0.50 & 4.53 & 0.0 & 1 & 194 \\
\hline 576 & 2 & 6 & 0.86 & 15.71 & 0.29 & 2.07 & 7.58 & 26.6 & 0.056 & 2.51 & 0.37 & 0.407 & 0.48 & 4.56 & 0. & 0. & 200 \\
\hline 576 & 2 & 6 & 0.96 & 15.81 & 0.42 & 2.04 & 7.54 & 26.2 & 0.058 & 2.43 & 0.36 & 0.401 & 0.49 & 4.59 & 0.0 & 4 & 204 \\
\hline 576 & 2 & 6 & 1.06 & 5.91 & 0.24 & 2.09 & 7.59 & 26.4 & 0.058 & 2.45 & 0.40 & 0.404 & 0.45 & 4.58 & 0. & 0. & 202 \\
\hline 576 & 2 & 6 & 1.16 & 16.01 & 0.39 & 2.08 & 7.52 & 26.2 & 0.056 & 2.44 & 0.41 & 0.406 & 0.49 & 4.59 & 0.0 & 0.042 & 191 \\
\hline 576 & 2 & 6 & 1.26 & 16.11 & 0.39 & 2.08 & 7.57 & 26.4 & 0.057 & 2.43 & 0.39 & 0.403 & 0.49 & 4.61 & 0. & 0. & 90 \\
\hline 576 & 2 & 6 & 1.36 & .21 & 0.44 & 2.1 & 7.6 & 26.3 & 0.053 & 2.52 & 0.36 & 0.416 & 0.46 & 4.69 & & & 6 \\
\hline 576 & 2 & 6 & 46 & 31 & 0.3 & 2.13 & 7.6 & 26.2 & 0.054 & 2.56 & 0.43 & 0.418 & 0.40 & 4.68 & & & 65 \\
\hline 576 & 2 & 7 & 0.06 & .41 & 0.45 & 2.10 & 7.66 & 26.3 & 0.054 & 2.56 & 0.39 & 0.4 & 0.43 & 4.72 & & & 65 \\
\hline 576 & 2 & 7 & 1 & 51 & 0.4 & 2.0 & 7.6 & 26 & 0.056 & 2.62 & 0. & 0.4 & 0. & 4.72 & & & 63 \\
\hline 576 & 2 & 7 & 2.2 & 51 & 0.4 & 2.1 & 7.6 & 26. & 0.054 & 2.49 & 0.38 & 0.2 & 0.49 & 67 & & & 86 \\
\hline 576 & 2 & 7 & 0.36 & & 0.3 & 2.15 & 7.6 & 26.3 & 0.057 & 2.49 & 0.43 & 0.410 & 0.46 & 4.66 & & & 181 \\
\hline 57 & 2 & 7 & & & 0.4 & 2.1 & 7.6 & & 0.056 & 2.46 & 0 & & & & & & 181 \\
\hline & 2 & 4 & & & & 2.04 & 7.67 & 26.2 & 0.052 & 2.63 & 0.39 & 0. & 0.35 & 4.83 & & & 162 \\
\hline 576 & 2 & 4 & 0.56 & & 0.2 & 2.03 & 7.63 & 26.1 & 0.052 & 2.56 & 0.36 & 0.424 & 0.40 & 4.79 & & & 166 \\
\hline 57 & 2 & 4 & 0.6 & & 0.28 & 2.0 & 7.66 & 26.0 & 0.048 & 2.62 & 0.37 & 0.429 & 0.36 & 4.89 & & & 154 \\
\hline 576 & 2 & 4 & 0.76 & 17 & 0.34 & 2.0 & 7.65 & 26.0 & 0.048 & 2.58 & 0.37 & 0.429 & 0.35 & 4.89 & & & 156 \\
\hline 576 & 2 & 4 & 0.86 & 17.36 & 0.3 & 2. & 7.63 & 26.0 & 0.050 & 2.54 & 0.41 & 0.428 & 0.38 & 4.82 & & & 155 \\
\hline $576 \mathrm{~B}$ & 2 & 4 & 0.96 & & 0.20 & 2. & 7.64 & 25.9 & 0.051 & 2.60 & 0.34 & 0.419 & 0.43 & 4.75 & & & 169 \\
\hline $576 \mathrm{~B}$ & 2 & 4 & 1.06 & 17.56 & 0.28 & 2.09 & 7.62 & 25.9 & 0.048 & 2.65 & 0.35 & 0.415 & 0.35 & 4.75 & 0.101 & 0.060 & 156 \\
\hline $576 \mathrm{~B}$ & 2 & 4 & 1.16 & 17.66 & 0.31 & 2.07 & 7.60 & 25.9 & 0.046 & 2.71 & 0.35 & 0.422 & 0.33 & 4.78 & 0.100 & 0.065 & 146 \\
\hline & 2 & 4 & 1.26 & 17.76 & 0.33 & 2.06 & 7.70 & 26.2 & 0.045 & 2.74 & 0.33 & 0.428 & 0.31 & 4.85 & 0.105 & & 141 \\
\hline $576 \mathrm{~B}$ & 2 & 4 & 1.36 & 17.86 & 0.28 & 2.11 & 7.81 & 26.4 & 0.046 & 2.69 & 0.32 & 0.427 & 0.34 & 4.81 & 0.126 & & 155 \\
\hline & 2 & 4 & 1.46 & 17 & 0.25 & 2.14 & 7.74 & 26. & 0.044 & 2.52 & 0.33 & 0.417 & 0.37 & 4.61 & 0.0 & & 173 \\
\hline & 2 & 5 & 0.06 & & 0. & 2. & 7.7 & 26 & 0.048 & 2.37 & 0. & 0.407 & & & & & 180 \\
\hline & 2 & 5 & 0.16 & & .20 & 2.28 & 7.67 & 26. & 0.050 & 2.20 & 0.35 & 0.391 & 0.40 & 4.24 & 0.083 & 0.053 & 193 \\
\hline
\end{tabular}




\begin{tabular}{|c|c|c|c|c|c|c|c|c|c|c|c|c|c|c|c|c|c|}
\hline$\stackrel{0}{\stackrel{0}{0}}$ & ్ㅣㅇ & 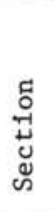 & 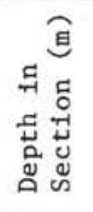 & 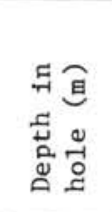 & $\mathrm{Na}$ & Mg & A1 & Si & $\mathrm{P}$ & K & $\mathrm{Ca}$ & Ti & $\mathrm{Mn}$ & $\mathrm{Fe}$ & $\mathrm{Ba}$ & S & $\mathrm{H}_{2} \mathrm{O}$ \\
\hline 76B & 2 & 5 & 25 & 25 & 0.24 & 36 & 3 & 6 & 9 & 01 & 6 & 81 & 0.36 & 3.99 & 0.075 & 0.044 & 190 \\
\hline $6 \mathrm{~B}$ & 2 & 5 & 36 & & & & & & & & & & & & & & \\
\hline $76 B$ & 2 & 5 & 0.4 & & 0. & & 7 & & 1 & & & & & & & & 6 \\
\hline $76 \mathrm{~B}$ & 2 & 5 & 0.56 & & & 2 & & & & & & & & & & & 159 \\
\hline $76 \mathrm{~B}$ & 2 & 5 & 0.66 & 18. & 0 & & & & & & & & & & & & 9 \\
\hline $76 \mathrm{~B}$ & 2 & 5 & 0.76 & 18 & & & & & & & & & & & & & 6 \\
\hline $76 \mathrm{~B}$ & 2 & 5 & 0.86 & 18 & & & & & & & & & & & & & \\
\hline 76 & 4 & 1 & 0.76 & & & & & & & & & & & & & & 8 \\
\hline 76 & 4 & 1 & 0.86 & 19 & & & & & & & & & & & & & 5 \\
\hline 76 & 4 & 1 & 0.96 & 19 & & & & & & & & & & & & & 4 \\
\hline 76 & 4 & 1 & 1.06 & & 0.43 & & & 0 & & & & & & & & & 173 \\
\hline 76 & 4 & 1 & 1.16 & 19.36 & 0.44 & & & 25.8 & & & & & & 4. & 8 & 8 & 175 \\
\hline 76 & 4 & 1 & 1.26 & 19.46 & 0.40 & & & 26. & & & & & & 4. & 8 & & 173 \\
\hline 76 & 4 & 1 & 1.36 & & 0.42 & 2. & 7.74 & 25.9 & & & 0 & 0 & & 4. & $\cdot$ & 2 & 171 \\
\hline 76 & 4 & 1 & 1.46 & & 0.47 & 2.1 & 7.82 & 26.0 & 0.056 & 2.00 & 0.44 & 0.4 & 0. & 4. & 0. & & 159 \\
\hline 76 & 4 & 2 & 0.05 & 19.75 & 0.41 & 2.10 & 7.8 & 26. & 0.056 & 2.61 & 0.46 & 0 & .55 & & & & 164 \\
\hline 10 & 4 & 2 & 0.16 & & 0.44 & 2.1 & 7.8 & & .0 & 2. & 0. & & & & & & 6 \\
\hline 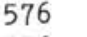 & 4 & 2 & 0.26 & & 0.43 & 2. & 7.8 & & & & 0 . & & & & & & 155 \\
\hline 576 & 4 & 2 & 0.36 & & 0.45 & & 7.8 & & & & & & & & & & 155 \\
\hline 576 & 4 & 2 & 0.46 & & 0.4 & 2.0 & 7.8 & & & & & & & & & & 48 \\
\hline 576 & 4 & 2 & 0.56 & 20.26 & & & & & & 2. & & & & & & & 60 \\
\hline 576 & 4 & 2 & 0.66 & 20.36 & 0.4 & 2. & & & & & & & & & & & 65 \\
\hline 576 & 4 & 2 & 0.76 & 46 & 0.4 & 2. & & & & & & & & & & & 9 \\
\hline 576 & 4 & 2 & 0.86 & 2 & 0. & 2 & & 2 & & & & & & & & & 53 \\
\hline 576 & 4 & 2 & 0.96 & 20 & & & & & & & & & & & & & 160 \\
\hline 576 & 4 & 2 & 1 & 20 & 0.5 & & & & & & & & & & & & 144 \\
\hline 576 & 4 & 2 & 1 & 2 & & & & & & & & & & & & & 148 \\
\hline 576 & 4 & 2 & 1 & 2 & 0.4 & & & & & & & & & & & & 150 \\
\hline 576 & 4 & 2 & 1 & 2 & & & & & & & & & & & & & 157 \\
\hline 5 & 4 & 2 & & & & & & & & & & & & 4. & & 4 & 152 \\
\hline 576 & 4 & 3 & 0 & & & & & & & & & & & 4. & 5 & 6 & 148 \\
\hline 576 & 4 & 3 & 16 & 36 & .47 & & & & 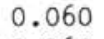 & 2. & ? & 5 & & 4. & & 7 & 14 \\
\hline 576 & 4 & 3 & 26 & 46 & .49 & & 7. & 5.6 & 3 & 2. & 0. & 5 & & 4. & & & 155 \\
\hline 576 & 4 & 3 & 36 & .56 & .46 & $2.0^{\prime}$ & 7. & 2 & 3 & 2. & 0. & .427 & 0 . & 4. & & & 1. \\
\hline 576 & 4 & 3 & 46 & 66 & .53 & 2.0 & 7.8 & 2 & 3 & 2. & 0 . & 0 & 0. & & & & 146 \\
\hline 576 & 4 & 3 & 0.56 & 76 & .48 & 2. & 7.8 & .6 & .0 & 2 . & 0.45 & 0. & 7 & 5. & & 6 & 148 \\
\hline 576 & 4 & 3 & 0.66 & .86 & .50 & 2.03 & 7.8 & 5.5 & C & 2. & 0.1 & 0.1 & 0.6 & 5. & 0.0 & 2 & 151 \\
\hline 576 & 4 & 3 & 0.76 & .96 & .50 & 2.03 & 7.8 & 5. & .0 & 2.5 & 0.49 & 0 . & 0. & 5. & & & 141 \\
\hline 5 & 4 & 3 & 0.86 & .06 & .46 & 2.02 & 7.8 & 5. & .0 & 2 & 0. & 0 & 0. & & & & 14 \\
\hline 576 & 4 & 3 & 0.96 & 22.16 & 0.50 & 2.06 & 7.9 & 5.8 & 0.06 & 2. & 0 & 0.447 & 0.6 & 5. & & & 144 \\
\hline 576 & 4 & 3 & 1.06 & .26 & 0.51 & 2.0 & 7.8 & 2 & 0.0 & 2 & 0 & 0.433 & & & & & 146 \\
\hline 576 & 4 & 3 & 1.16 & 36 & 0.49 & 2.0 & 7.8 & . & . & 2. & 0. & 0. & & & & & 140 \\
\hline 576 & 4 & 3 & 1.26 & 2 & .4 & 2.0 & 7.8 & 5.6 & 0.06 & 2. & 0.1 & 0.442 & & 5. & & & 146 \\
\hline 5 & 4 & 3 & 1.36 & .56 & 0.4 & . 0 & 7.8 & . & 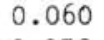 & 2. & 0 . & 0. & & & & & 154 \\
\hline 576 & 4 & 3 & 1.46 & .66 & $.4 C$ & .0 & 7.8 & 25.5 & 0.05 & 2. & 0 & 0.439 & & 5. & & & 8 \\
\hline 5 & 4 & 4 & 0.06 & 2 & .4 & 0 & 7 & 25.6 & 0.06 & 2. & 0 & & & & & & 0 \\
\hline 5 & 4 & 4 & 16 & & 4 & 0 & 7.8 & 5. & & 2.8 & 0.1 & & & & & & +3 \\
\hline 576 & 4 & 4 & 26 & 2 & .4 & 2.0 & 7.8 & 5. & 0 & 2.65 & 0.2 & & & & & & 143 \\
\hline $5^{\prime}$ & 4 & 4 & 36 & 2 & $.48 \mathrm{r}$ & .0 & 7 & 5.3 & 0 t & 2. & 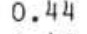 & & & 5 & & & 38 \\
\hline 57 & 4 & 4 & 0 & 2 & .45 & 0 & 7 & 5.12 & 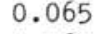 & 2. & 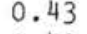 & & & & & & 134 \\
\hline 576 & 4 & 4 & 56 & 2 & 48 & 0 & 7. & 25. & 3 & 2. & 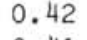 & & & & & & 35 \\
\hline 576 & 4 & 4 & 0 & 2. & 0.53 & 2.01 & 7.7 & 25. & 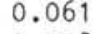 & 2. & 0 & 0.437 & & & & & 127 \\
\hline 576 & 4 & 4 & & & 5 & 9 & & & & & & & & & & & 131 \\
\hline 576 & 4 & 4 & 0.86 & 23.56 & 0.53 & 1.98 & 7.8 & & 3 & 2. & 0.42 & & & & & 0.071 & 127 \\
\hline 576 & 4 & 4 & 0.96 & 23.66 & 0.51 & 1.99 & 7.8 & & 0.068 & 2.73 & 0.43 & & & 4.92 & & 0. & 134 \\
\hline 57 & 4 & 4 & 06 & & & & & & & & & & & 4.91 & & & 131 \\
\hline 57 & 4 & 4 & 16 & & .4 & 1.98 & & & & 2.71 & 0.42 & & & & & & 138 \\
\hline 5 & 4 & 4 & 1.26 & 23.9 & 0.45 & 1.98 & 7.8 & 25 . & & 2.69 & 0.43 & & & & & & 140 \\
\hline 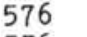 & 4 & 4 & 1 & 24. & 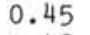 & 1. & 7.8 & & & 2.6 & 0.4 & & & & & & 135 \\
\hline & 4 & 4 & & & & & & & & & & & & & & & 137 \\
\hline 10 & 4 & 5 & 0.06 & 24.26 & 0.46 & 1.95 & 7.91 & 25.5 & 0.060 & 2.65 & 0.44 & 0.444 & & - & 0.076 & 0.049 & 138 \\
\hline
\end{tabular}


Table 5. (Continued).

\begin{tabular}{|c|c|c|c|c|c|c|c|c|c|c|c|c|c|c|c|c|c|}
\hline 공 & $\stackrel{\leftrightarrow}{\circ}$ & $\begin{array}{l}\text { 号 } \\
\text { İ } \\
\text { ¿ } \\
\text { ஸे }\end{array}$ & 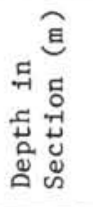 & 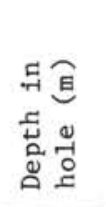 & $\mathrm{Na}$ & $\mathrm{Mg}$ & A1 & Si & $\mathrm{P}$ & K & $\mathrm{Ca}$ & $\mathrm{T} 1$ & Mn & $\mathrm{Fe}$ & $\mathrm{Ba}$ & $\mathrm{s}$ & $\mathrm{H}_{2} \mathrm{O}$ \\
\hline 576 & 4 & 5 & 0.16 & 36 & 0.44 & 1.98 & 7.91 & .6 & 60 & 2.68 & 0.40 & 440 & 66 & 5.00 & 0.077 & .046 & 139 \\
\hline 576 & 4 & 5 & 0.26 & 46 & 0.43 & 1 & 7 & 2 & 67 & & & 3 & & & .079 & & 10 \\
\hline 576 & 4 & 5 & 0.36 & 4.56 & 0.47 & 2 & & 25.5 & 0.070 & & & & 1 & 8 & 0.080 & 0.056 & 133 \\
\hline 576 & 4 & 5 & 0.46 & 4.66 & 0.49 & 2.01 & 7. & & 0.072 & & & & & & .082 & & 132 \\
\hline 576 & 4 & 5 & 0.56 & 24.76 & 0.46 & 2.02 & & & & & & & & & 0.087 & 51 & 136 \\
\hline 576 & 4 & 5 & 0.66 & 24.86 & 0.43 & 1.98 & & 2 & 0 & & & & & & 0.081 & .036 & 135 \\
\hline 576 & 4 & 5 & 0.76 & 24.96 & 0.44 & 1.99 & & & & & & 0.4 & & 4.96 & 0.080 & 0.049 & 134 \\
\hline 576 & 4 & 5 & 0.86 & 25.06 & 0.41 & 2.00 & 7.96 & 25.6 & 0. & 2.71 & 0.40 & 0.442 & 0.75 & 4.99 & 0.077 & .049 & 141 \\
\hline 576 & 4 & 5 & 0.96 & 25.16 & 0.41 & 2.00 & 7.94 & 25.5 & 0.071 & 2.71 & 0.38 & 0.440 & 0.72 & 4.97 & 0.081 & 0.053 & 143 \\
\hline 576 & 4 & 5 & 1.06 & 25.26 & 0.45 & 1.99 & 7.89 & 25.4 & 0.067 & 2.70 & 0.36 & 0.430 & 0.67 & 4.92 & 0.077 & & 134 \\
\hline 576 & 4 & 5 & 1.16 & $25 \cdot 36$ & 0.45 & 2.01 & 7.93 & 25.4 & 0.070 & 2.72 & 0.40 & 0.434 & 0.72 & 4.91 & 0.078 & .069 & 137 \\
\hline 576 & 4 & 5 & 1.26 & 25.46 & 0.46 & 2.01 & 7.8 & 25.4 & 0.067 & 2.73 & 0.39 & 0.437 & 0.71 & 4.93 & 0.080 & .063 & 129 \\
\hline 576 & 4 & 5 & 1.36 & 25.56 & 0.49 & 2.00 & 7.8 & 25.6 & 0.068 & 2.80 & 0.39 & 0.445 & 0.54 & 5. & 0.077 & .059 & 121 \\
\hline 576 & 4 & 5 & 1.46 & 25.66 & 0.50 & 1.99 & 7.81 & 25.5 & 0.066 & 2.83 & 0.39 & 0.4 & 0.60 & 4.5 & 0.079 & 62 & 1.17 \\
\hline 576 & 4 & 6 & 0.06 & 25.76 & 0.51 & 2.01 & 7.8 & 25.7 & 0.066 & 2.83 & 0.39 & 0.450 & 0.67 & 5.05 & 0.080 & 0.053 & 117 \\
\hline 576 & 4 & 6 & 0.16 & 25.86 & 0.53 & 2.00 & 7.7 & 25.4 & 0.069 & 2.83 & 0.40 & 0.446 & & & 0.079 & & 110 \\
\hline 576 & 4 & 6 & 0.26 & 25.96 & 0.51 & 2.01 & 7.8 & 25.6 & 0.073 & 2.83 & 0.39 & 0.1 & 1 & & 0.076 & 57 & 116 \\
\hline 576 & 4 & 6 & 0.36 & 26.06 & 0.45 & 2.03 & 7.86 & 25.7 & 0.079 & 2.8 & 0.3 & 0.4 & 9 & & 0.082 & & 117 \\
\hline 576 & 4 & 6 & 0.46 & 26.16 & 0.51 & 2.03 & 7.7 & & 0 & 2 & & & & & 0.084 & 1 & 109 \\
\hline 576 & 4 & 6 & 0.56 & 26.26 & 0.50 & 2.1 & 7.5 & 25.1 & 0.1 & 2.80 & 0. & 0. & 5 & 5 & 0.092 & 57 & 115 \\
\hline 576 & 4 & 6 & 0.66 & 26.36 & 0.45 & 2.18 & 7.42 & 25.3 & 0.163 & 2.77 & 0.59 & 0.5 & 0.99 & 5.63 & 0.110 & 45 & 124 \\
\hline 576 & 4 & 6 & 0.76 & 26.46 & 0.47 & 2.1 & 7.2 & 25.0 & 0.167 & 2. & 0. & 0. & & & 0.110 & & 124 \\
\hline 576 & 4 & 6 & 0.86 & 26.56 & 0.46 & 2.1 & $7 \cdot 3$ & 25.1 & 0.157 & 2.76 & 0.58 & 0.5 & 0 & 5. & 0.106 & 45 & 123 \\
\hline 576 & 4 & 6 & 0.96 & 26.66 & 0.46 & 2.17 & 7.28 & 25.1 & 0.1 & 2.76 & 0.64 & 0.5 & 0 & & 0.115 & 54 & 129 \\
\hline 576 & 4 & 6 & 1.06 & 26.76 & 0.45 & 2.1 & 7.1 & 24.9 & 0. & 2.73 & 0. & 0. & 0. & & 0.117 & 53 & 131 \\
\hline 576 & 4 & 6 & 1.16 & 26.86 & 0.46 & 2.1 & 7.1 & 24.8 & 0. & 2. & & 0. & & & 0.115 & 57 & 127 \\
\hline 576 & 4 & 6 & 1.26 & 26.96 & 0.1 & 2.1 & 7. & 25 & 0. & 2 & & & & & 0.114 & & 128 \\
\hline 576 & 4 & 6 & 1.36 & 27.06 & 0.4 & 2. & 7.2 & 25 & 0. & 2. & 0 & & & & 0.115 & & 129 \\
\hline 576 & 4 & 6 & 1.46 & 6 & 0.1 & 2 & 7. & 24 & 0. & 2. & 0. & 0 & & & 112 & 69 & 132 \\
\hline 576 & 4 & 7 & 0.06 & 6 & 0. & & & & 0. & 2 & & & & & 0.117 & 6 & 128 \\
\hline 576 & 4 & 7 & 0 & 6 & 0 & & & & & & & & & & 16 & 62 & 130 \\
\hline 576 & 4 & 7 & 0 & 6 & 0 . & & & & & & & & & & 7 & 66 & 129 \\
\hline 576 & 4 & 7 & 0.36 & 6 & 0 . & 2.15 & 7. & 2 & 0. & 7 & 0 & 0 & 0 & 5 & 115 & 82 & 127 \\
\hline 576 & 4 & 7 & 0.46 & .66 & 0.45 & 2. & 7. & 2. & 6 & 3 & ? & 0 & 1.00 & 5 & 0.117 & .076 & 128 \\
\hline 576B & 3 & 6 & 0.26 & 27.92 & 0.44 & 2.06 & 7.86 & 25.1 & 0.093 & 2.77 & 0.44 & 0.1 & 0.95 & 5 & 0.082 & 0.053 & 118 \\
\hline $576 \mathrm{~B}$ & 3 & 6 & 0.46 & 28.12 & 0.49 & 2.08 & 7.78 & 24.7 & 0.109 & 2.78 & 0.50 & 0.45 & 0.96 & 5.26 & 0.080 & .076 & 119 \\
\hline 576B & 3 & 6 & 0.66 & 28.32 & 0.48 & 2.08 & 7.85 & 24.9 & 0.103 & 2.82 & 0.50 & 0.464 & 1.04 & 5.35 & 0.078 & 0.062 & 118 \\
\hline $576 \mathrm{~B}$ & 3 & 6 & 0.86 & 28.52 & 0.48 & 2.03 & 7.77 & 24.8 & 0.100 & 2.80 & 0.47 & 0.45 & 0.99 & 5.23 & 0.079 & 8 & 113 \\
\hline $576 \mathrm{~B}$ & 3 & 6 & 1.06 & 28.72 & 0.51 & 2.07 & 7.85 & 24.9 & 0.107 & 2.85 & 0.49 & 0.465 & 1.03 & 5. & 0.081 & 0.067 & 113 \\
\hline $76 \mathrm{~B}$ & 3 & 6 & 1.26 & 28.92 & 0.50 & 2.03 & $7.7^{\circ}$ & 24.8 & .1 & 2.84 & 0.52 & 0.4 & 1.05 & 5 & 0.072 & 8 & 115 \\
\hline $576 \mathrm{~B}$ & 3 & 6 & 1.46 & 29.12 & 0.50 & 2.0 & 7.7 & 24.4 & 0.117 & 2.75 & 0.53 & 0.4 & 1.0 & 5 & 0.072 & 0 . & 119 \\
\hline $76 \mathrm{~B}$ & 3 & 7 & 0.06 & ? & 0.51 & 2.0 & 7.7 & 4.5 & 0.122 & 2.76 & 0.53 & .2 & 1. & 5.34 & 0.075 & 0. & 119 \\
\hline $6 \mathrm{~B}$ & 3 & 7 & 0.26 & 42 & 0.5 & 2.0 & 7.7 & 4.3 & 0.129 & 2.7 & 0.57 & .4 & 1.2 & 5.26 & 0.074 & 0 . & 124 \\
\hline $76 \mathrm{~B}$ & 3 & 7 & 0.46 & 52 & 0.5 & .9 & 7.80 & 24.3 & 0.1 & 2.7 & 0.55 & 0 & 1.21 & 5 & 0.072 & 1 & 124 \\
\hline 76 & 4 & 1 & 0.74 & & $0.6^{\prime}$ & 9 & 7.9 & 24.2 & 7 & 2.7 & 0. & 0.445 & 1. & 5. & 0.073 & 7 & 127 \\
\hline $6 \mathrm{~A}$ & 4 & 1 & 0.78 & 8 & 0.7 & 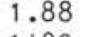 & 7 & 24 & 3 & 2. & & & & & 0.072 & & \\
\hline & 4 & 1 & 9 & & & & & 24.1 & 36 & 2.73 & 0.50 & 0 & 1 & & 0.071 & 41 & 121 \\
\hline $576 \mathrm{~A}$ & 4 & 1 & 1.39 & & 0.6 & 1.9 & 8. & 24.4 & 0.123 & 2.72 & 0.43 & 0. & 1. & & 0.071 & 42 & 129 \\
\hline & 4 & 2 & 0 & 59 & 0.6 & 2. & 7.8 & 23.9 & 0.127 & 2.79 & & & & & 0.073 & & \\
\hline 576 & 5 & 5 & 0.56 & 06 & 0 & 2 & & 24.0 & 0.150 & 2.65 & & & & & 0.074 & 73 & 134 \\
\hline 576 & 5 & 5 & 0.66 & 16 & 0.51 & 2.06 & 7.89 & 23.8 & 0.156 & 2.63 & 0.61 & 0.1 & 1. & & 0.074 & 64 & 134 \\
\hline 576 & 5 & 5 & 0.76 & 26 & 0.57 & 2.02 & 7.9 & 24.0 & 0.155 & 2.72 & 0.61 & & & & 0.072 & 57 & 129 \\
\hline 5 & 5 & 5 & & & 0.6 & 1.91 & 7.83 & 23.6 & 0.151 & 2.73 & 0.61 & 0. & 1.40 & 55 & 0.067 & 0.059 & 139 \\
\hline 576 & 5 & 5 & 0.96 & 31.46 & 0.54 & 2.02 & 7.79 & 23.8 & 0.172 & 2.60 & 0.67 & 0.4 & 1.61 & & 0.068 & 0.060 & 146 \\
\hline & 4 & 3 & 1.06 & 31.56 & 0.46 & 2.00 & 7.76 & 23.7 & 0.180 & 2.58 & 0.66 & 0.435 & 1.57 & & 0.070 & 0.063 & 151 \\
\hline $576 \mathrm{~B}$ & 4 & 3 & 1.26 & 31.76 & 0.52 & 1.96 & 7.69 & 23.7 & 0.198 & 2.69 & 0.74 & & 1.68 & & 0.074 & 0.072 & 139 \\
\hline $576 \mathrm{~B}$ & 4 & 3 & 1.46 & 31.96 & 0.42 & 1.99 & 7.72 & 23.4 & 0.203 & 2.56 & 0.70 & 0.454 & 1.83 & 5.61 & 0.071 & & 159 \\
\hline & 4 & 4 & 0.06 & & 0.44 & 2.01 & 7. & 23.4 & 0.205 & 2.61 & 0.75 & 0.4 & & & 0.073 & & 149 \\
\hline & 4 & 4 & 20 & & 0.40 & 1.97 & & 4 & 215 & 2.59 & 0.71 & 0.443 & 6 & & 0.072 & 58 & 152 \\
\hline & 4 & 4 & 0.46 & .40 & 0.42 & 1.98 & 7.75 & 23. & & 2.65 & & & & & 0.072 & & 138 \\
\hline & 4 & 4 & & & 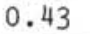 & $\begin{array}{l}91 \\
\end{array}$ & 19 & 3.7 & 0.172 & 2.77 & & 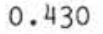 & & & 0.072 & 0.060 & 128 \\
\hline
\end{tabular}




\begin{tabular}{|c|c|c|c|c|c|c|c|c|c|c|c|c|c|c|c|c|c|}
\hline 옴 & $\stackrel{\mathscr{H}}{\circ}$ & 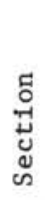 & 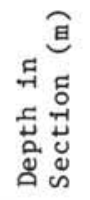 & 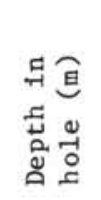 & $\mathrm{Na}$ & $\mathrm{Mg}$ & A1 & Si & $\mathrm{P}$ & K & $\mathrm{Ca}$ & $\mathrm{Ti}$ & Mn & $\mathrm{Fe}$ & $\mathrm{Ba}$ & S & $\mathrm{H}_{2} \mathrm{O}$ \\
\hline $576 B$ & 4 & 4 & 0.86 & 32.86 & 0.43 & 2.02 & 7.87 & 24.0 & 0.158 & 2.81 & 0.55 & 0.442 & 1.42 & 5.15 & 0.071 & 0.067 & 127 \\
\hline $576 \mathrm{~B}$ & 4 & 4 & .06 & 33.06 & 0.43 & 1.98 & 7.73 & 23.6 & 0.170 & 2.70 & 0.62 & 0.446 & 1.61 & .22 & 0.070 & .074 & 142 \\
\hline $576 \mathrm{~B}$ & 4 & 4 & 1.26 & 33.26 & 0.44 & 1.98 & 7.72 & 23.5 & 0.193 & 2.62 & 0.68 & 0.450 & 1.83 & .31 & 0.074 & 0.067 & 148 \\
\hline $576 \mathrm{~B}$ & 4 & 4 & 1.46 & 33.46 & 0.42 & 1.95 & 7.71 & 23.3 & 0.232 & 2.62 & 0.77 & 0.446 & 2.04 & 5.25 & 0.074 & 0.073 & 154 \\
\hline $576 \mathrm{~B}$ & 4 & 5 & 0.06 & 33.56 & 0.42 & 1.99 & 7.77 & 23.5 & 0.214 & 2.67 & 0.75 & 0.457 & 2.01 & 5.26 & 0.074 & 0.076 & 153 \\
\hline $576 \mathrm{~B}$ & 4 & 5 & 0.26 & 33.76 & 0.40 & 1.99 & 7.59 & 23.0 & 0.262 & 2.55 & 0.90 & 0.462 & 2.19 & 5.33 & 0.074 & .083 & 148 \\
\hline $576 \mathrm{~B}$ & 4 & 5 & 0.46 & 33.96 & 0.41 & 1.96 & 7.79 & 23.4 & 0.250 & 2.53 & 0.76 & 0.458 & 2.07 & 5.46 & 0.075 & 0.043 & 157 \\
\hline $576 \mathrm{~B}$ & 4 & 5 & 0.66 & 34.16 & 0.40 & 1.99 & 7.54 & 22.9 & 0.288 & 2.57 & 0.95 & 0.455 & 2.03 & 5.33 & 0.073 & 0.094 & 153 \\
\hline $76 \mathrm{~B}$ & 4 & 5 & 0.86 & 34.36 & 0.34 & 2.03 & 7.57 & 23.1 & 0.289 & 2.61 & 0.97 & 0.463 & 2.05 & & 0.074 & 86 & 150 \\
\hline 576B & 4 & 5 & 1.06 & 34.56 & 0.35 & 2.04 & 7.54 & 23.1 & 0.312 & 2.62 & 1.01 & 0.468 & 2.15 & 5.46 & 0.077 & 0.081 & 149 \\
\hline $576 \mathrm{~B}$ & 4 & 5 & 1.26 & 34.76 & 0.31 & 2.07 & 7.38 & 23.0 & 0.443 & 2.59 & 1.28 & 0.457 & 2.20 & 5.54 & 0.077 & 0.086 & 154 \\
\hline $76 \mathrm{~B}$ & 4 & 5 & 1.46 & 34.96 & 0.32 & 2.07 & 7.29 & 22.7 & 0.458 & 2.59 & 1.37 & 0.455 & 2.13 & 5.47 & 0.076 & 0.095 & 151 \\
\hline $576 \mathrm{~B}$ & 4 & 6 & 0.06 & 35.06 & 0.33 & 2.06 & 7.35 & 22.8 & 0.449 & 2.61 & 1.36 & 0.454 & 2.12 & 48 & $0.0^{\circ}$ & & 148 \\
\hline $576 B$ & 4 & 6 & 0.26 & 35.26 & 0.34 & 2.07 & 7.30 & 22.7 & 0.457 & 2.61 & 1.39 & 0.452 & 2.12 & & 0.0 & & 49 \\
\hline $576 \mathrm{~B}$ & 4 & 6 & 0.46 & & 0.34 & 2.01 & 7.11 & 22.2 & 0.446 & 2.55 & 1.36 & 0 & 2 & & & & 51 \\
\hline$B$ & 4 & 6 & 0.66 & 35 & 0. & 2. & 7. & 22 & 0 & 1 & 1. & 0.452 & 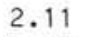 & & & & 44 \\
\hline $576 \mathrm{~B}$ & 4 & 6 & 0.86 & 35 & 0. & 2 & 7 & 22 & 5 & 1 & 1 & 0.4 & 0 & & & & 48 \\
\hline 576 & 4 & 6 & 1. & & 0.34 & 2.06 & 7. & 22.7 & 0.1 & 2. & 1. & & 7 & & & & 144 \\
\hline $576 \mathrm{~B}$ & 4 & 6 & 1. & & 0. & 2. & 7. & 22 & 0 & 2 & 9 & & 7 & & & & 147 \\
\hline $576 \mathrm{~B}$ & 4 & 6 & 1 & & 0. & 2. & 7 & 2 & 0 & 8 & & & & & & & 125 \\
\hline $576 \mathrm{~B}$ & 4 & 7 & 0.0 & & 0. & 2. & 7 & 23 & 0 & 2 & & & & & & & 129 \\
\hline 57 & 4 & 7 & 0 & & 0. & 1. & 7 & 23 & 0 & 1 & 0 & & 5 & & 2 & & 129 \\
\hline $576 \mathrm{~B}$ & 4 & 7 & 0.4 & 3 & 0. & 1. & 7 & 23 & 0 & 2 & & & 6 & & & & 129 \\
\hline 576 & 6 & 3 & 0 & & 0. & 2. & 7 & 23 & 3 & 3 & & & & & & & 122 \\
\hline 576 & 6 & 3 & 0. & & 0. & 2. & 8 & 23 & 5 & 1 & 8 & & 1 & & 3 & 3 & 122 \\
\hline 576 & 6 & 3 & 0. & & 0. & 2. & 7. & 23 & 0 & 9 & & & & & & 5 & 124 \\
\hline 76 & 6 & 3 & 1.06 & 36 & 0.38 & 2.06 & 8.00 & 23.9 & 0. & 3. & 6 & 1 & 1.50 & 8 & 0. & 71 & 125 \\
\hline 576 & 6 & 3 & 1.16 & 38.46 & 0.38 & 2.07 & 8.01 & 24.0 & 0.319 & 3.02 & 0.88 & 0.3 & 1.53 & 5. & 0. & 0.079 & 121 \\
\hline 576 & 6 & 3 & 1.26 & 56 & 0. & 2.07 & 7.97 & 24.0 & 0. & 2.96 & 2 & 0.384 & 1.53 & 8 & 0.0 & 0.066 & 125 \\
\hline 576 & 6 & 3 & 1.36 & 66 & 0.37 & 2.06 & 7.95 & 23.8 & 0.297 & 2.92 & 0.86 & 0. & 1.66 & 4.96 & 0. & 0. & 123 \\
\hline 576 & 6 & 3 & 1.46 & 76 & 0.36 & 2.02 & 7.88 & 23.6 & 0.294 & 2.86 & 0.85 & 0. & 1.70 & 4.91 & 0.0 & 9 & 127 \\
\hline 576 & 6 & 4 & 0.06 & 38.86 & 0.34 & 2.11 & 7.84 & 23.5 & 0.301 & 2.90 & 0.87 & 0.440 & 1.58 & 5.15 & 0. & 0. & 123 \\
\hline 576 & 6 & 4 & 0.16 & 96 & 0.33 & 2.14 & 7.94 & 23.7 & 0.2 & 2.92 & 0.81 & 0.473 & 1. & 8 & 0.0 & 0. & 119 \\
\hline 576 & 6 & 4 & 0.26 & 39.06 & 0.36 & 2.15 & 7.83 & 23.4 & 0.311 & 2.84 & 0.84 & 0.487 & 1.50 & 5.33 & 0.073 & 0.070 & 121 \\
\hline 576 & 6 & 4 & 0.36 & 16 & 0.36 & 2.13 & 7.96 & 23.6 & 0.290 & 2.82 & 0.80 & 0.490 & 1.51 & 5.32 & 0.072 & 0.071 & 122 \\
\hline 576 & 6 & 4 & 0.46 & 39.26 & 0.37 & 2.10 & 7.94 & 23.6 & 0.311 & 2.77 & 0.87 & 0.500 & 1.53 & 5.31 & 0.072 & 0.074 & 124 \\
\hline 576 & 6 & 4 & 0.56 & 39.36 & 0.39 & 2.04 & 7.91 & 23.4 & 0.360 & 2.64 & 0.99 & 0.532 & 1.57 & 5.40 & 0.071 & 0.076 & 129 \\
\hline 576 & 6 & 4 & 0.66 & 39.46 & 0.40 & 1.98 & 7.89 & 23.4 & 0.352 & 2.68 & 0.95 & 0.550 & 1.45 & 5.53 & 0.071 & 0.078 & 128 \\
\hline 576 & 6 & 4 & 0.76 & 39.56 & 0.38 & 1.95 & 7.92 & 23.6 & 0.325 & 2.81 & 0.91 & 0.5 & 1.48 & 6 & 0.072 & 69 & 132 \\
\hline 576 & 6 & 4 & 0.86 & 66 & 0.37 & 2.02 & 7.90 & 23.5 & 0.364 & 2.65 & 0.98 & 0.595 & 1.59 & 5.66 & 0.073 & 0.076 & 139 \\
\hline 576 & 6 & 4 & 0.96 & 76 & 0.34 & 2.01 & 7.81 & 23.0 & 0.327 & 2.55 & 0.91 & 0.5 & 1. & 5 & 0.0 & 9 & 140 \\
\hline 576 & 6 & 4 & 1.06 & 86 & 0.38 & 2.01 & 7.87 & 23.1 & 0.350 & 2.59 & 0.94 & 0.6 & 1.74 & 0 & 0.0 & 5 & 137 \\
\hline 576 & 6 & 4 & 1.16 & 96 & 0.35 & 2.03 & 7.88 & 23.1 & 0.345 & 2.64 & 0 & 0. & 1.66 & 0 & 0. & & 38 \\
\hline 576 & 6 & 4 & 1.26 & 06 & 0.34 & 2.01 & 7.86 & 23.2 & 0.301 & 2.69 & 0.83 & 0. & 1.68 & 88 & 0. & 3 & 140 \\
\hline 576 & 6 & 4 & 1.36 & 16 & 0.36 & 2.03 & 7.89 & 23.2 & 0.324 & 2.62 & 0.86 & 0. & 1. & 2 & 0. & & 140 \\
\hline 57 & 6 & 4 & 1.46 & 26 & 0.39 & 2.05 & 7.87 & 23.1 & 0.325 & 2.65 & 0 & 0. & 1.74 & 5 & 0. & & 144 \\
\hline 576 & 6 & 5 & 0.06 & 36 & 0.43 & 2.03 & 7.70 & 22.7 & 0.319 & 2.63 & 0.89 & 0. & 6 & & 5 & 92 & 140 \\
\hline 576 & 6 & 5 & 0.16 & 46 & 0. & 2.04 & 7.79 & 23.0 & 0.303 & 3 & 8 & & 57 & 93 & 0.076 & & 138 \\
\hline 576 & 6 & 5 & 0.26 & 40.56 & 0.39 & 2.07 & 7.90 & 23.3 & 14 & 2.67 & 0.89 & 0.608 & 1.68 & 01 & 0.077 & & 139 \\
\hline 576 & 6 & 5 & 0.36 & 40.66 & 0.43 & 2.07 & 7.92 & 23.3 & 0.312 & 2.69 & 0.89 & 0.612 & 1.73 & 02 & 0.075 & & 141 \\
\hline 576 & 6 & 5 & 0.46 & & 0.39 & 2.04 & & 23.2 & 0.335 & 2.64 & & & 1.69 & & & & 139 \\
\hline 576 & 6 & 5 & 0.56 & 40.86 & 0.40 & 2.05 & 7.82 & 23.0 & 0.308 & 2.65 & 0.89 & 0.599 & 1.68 & & 0.075 & 0.083 & 141 \\
\hline 576 & 6 & 5 & 0.66 & 40.96 & 0.41 & 2.07 & 7.89 & 23.3 & 0.309 & 2.66 & 0.88 & 0.607 & 1.70 & 6.03 & 0.075 & 0.079 & 137 \\
\hline 576 & 6 & 5 & 0.76 & 41.06 & 0.41 & 2.07 & 7.86 & 23.2 & 0.301 & 2.66 & 0.86 & 0.598 & 1.67 & 6.02 & 0.076 & 0.086 & 137 \\
\hline 576 & 6 & 5 & 0.86 & 41.16 & 0.41 & 2.04 & 7.85 & 23.2 & 0.298 & 2.68 & 0.85 & 0.593 & 1.57 & 5.93 & 0.075 & 0.083 & 137 \\
\hline 576 & 6 & 5 & 0.96 & 41.26 & 0.36 & 2.01 & 7.93 & 23.5 & 0.294 & 2.77 & 0.79 & 0.598 & 1.36 & 5.80 & 0.072 & 0.064 & 132 \\
\hline 576 & 6 & 5 & 1.06 & 41.36 & 0.36 & 2.04 & 7.94 & 23.6 & 0.292 & 2.78 & 0.81 & 0.595 & 1.34 & & 0.070 & 0.074 & 130 \\
\hline 75 & 6 & 5 & 1.16 & 41.46 & 0.37 & 2.04 & 7.93 & 23.5 & 0.293 & 2.79 & 0.82 & 0.599 & 1.35 & 5.81 & 0.070 & 0.080 & 132 \\
\hline 576 & 6 & 5 & 1.26 & 41.56 & 0.35 & 2.02 & 7.96 & 23.6 & 0.290 & 2.77 & 0.80 & 0.598 & 1.40 & 5.80 & 0.070 & 0.062 & 130 \\
\hline 75 & 6 & 5 & 1.36 & 41.66 & 0.34 & 2.05 & 7.86 & 23.3 & 0.299 & 2.77 & 0.80 & & 1.38 & & 0.072 & 0.071 & 133 \\
\hline 576 & 6 & 5 & 1.46 & 41.76 & 0.25 & 2.15 & 7.71 & 23.1 & 0.332 & 2.74 & 0.84 & 0.677 & 1.46 & 6.21 & 0.075 & 0.074 & 138 \\
\hline
\end{tabular}




\begin{tabular}{|c|c|c|c|c|c|c|c|c|c|c|c|c|c|c|c|c|c|}
\hline ํㅜㅇ & ثّ & 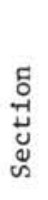 & 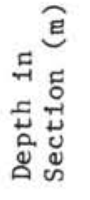 & 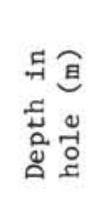 & $\mathrm{Na}$ & Mg & Al & Si & P & K & $\mathrm{Ca}$ & $\mathrm{T} 1$ & Mn & $\mathrm{Fe}$ & $\mathrm{Ba}$ & $\mathrm{S}$ & $\mathrm{H}_{2} \mathrm{O}$ \\
\hline 576 & 6 & 6 & 0.06 & 41.86 & 0.35 & 2.11 & 7.69 & 23.1 & 0.316 & 2.75 & 0.85 & 0.721 & 1.44 & 6.40 & 0.075 & 7 & 140 \\
\hline 576 & 6 & 6 & 0.16 & 41.96 & 0.34 & 2.11 & 7.67 & 3.0 & 360 & 2.72 & 0.89 & 0.723 & 1.40 & .50 & .075 & & 140 \\
\hline 576 & 6 & 6 & 0.26 & 42.06 & 0.37 & 2.10 & 7.66 & 23.0 & 0.319 & 2.74 & 0.87 & 0.727 & 1.40 & 6.50 & 0.074 & 0.077 & 139 \\
\hline 576 & 6 & 6 & 0.36 & 42.16 & 0.42 & 2.09 & 7.63 & 22.9 & 0.322 & 2.72 & 0.86 & 0.724 & 1.36 & 6.50 & 0.074 & 0.062 & 141 \\
\hline 576 & 6 & 6 & 0.46 & 42.26 & 0.39 & 2.08 & 7.73 & 23.2 & 0.314 & 2.84 & 0.87 & 0.748 & 1.36 & 6.56 & 0.075 & 0.059 & 138 \\
\hline 576 & 6 & 6 & 0.56 & 42.36 & 0.44 & 2.08 & 7.79 & 23.5 & 0.311 & 3.03 & 0.85 & 0.741 & 1.29 & 6.32 & 0.075 & 64 & 130 \\
\hline 576 & 6 & 6 & 0.66 & 42.46 & 0.43 & 2.07 & 7.76 & 23.6 & 0.287 & 3.12 & 0.81 & 0.742 & 1.21 & 6.28 & 0.074 & 0.067 & 127 \\
\hline 576 & 6 & 6 & 0.76 & 42.56 & 0.43 & 2.08 & 7.78 & 23.8 & 0.295 & 3.16 & 0.81 & 0.751 & 1.18 & & 0.075 & & 125 \\
\hline 576 & 6 & 6 & 0.86 & 42.66 & 0.40 & 2.12 & 7.70 & 23.7 & 0.302 & 3.17 & 0.81 & 0.730 & 1.17 & 6.22 & 0.074 & 0. & 128 \\
\hline 576 & 6 & 6 & 0.96 & 42.76 & 0.38 & 2.08 & 7.53 & 23.3 & 0.248 & 3.12 & 0.69 & 0.656 & 1.14 & & 0.072 & & 119 \\
\hline 576 & 6 & 6 & 1.06 & 42.86 & 0.41 & 2.18 & 7.63 & 23.8 & 0.230 & 3.20 & 0.65 & 0.710 & 1.10 & & 0.073 & & 115 \\
\hline 576 & 6 & 6 & 1.16 & 42.96 & 0.37 & 2.17 & 7.73 & 24.0 & & 3.18 & 0.70 & & 1.17 & 1 & 0.0 & 0.064 & 115 \\
\hline 576 & 6 & 6 & 1.26 & 43.06 & 0.34 & 2.17 & 7.72 & 24.0 & & 3.18 & 0.72 & 0.604 & 1.21 & & 75 & & 111 \\
\hline 576 & 6 & 6 & 1.36 & 43.16 & 0.33 & 2.18 & 7.65 & 23.9 & & 3.16 & & 0.569 & 1.15 & & & & 106 \\
\hline 576 & 6 & 6 & 1.4 & 43.26 & 0.31 & 2.26 & 7.71 & 24.1 & 0. & 3. & 0.6 & 0.593 & 1. & & 0. & & 109 \\
\hline 576 & 6 & 7 & 0.06 & 43.36 & 0.31 & 2.23 & 7.50 & 23.7 & & 3.12 & 0. & 0.5 & 1.13 & & & & 114 \\
\hline 10 & 6 & 7 & 0.1 & 43.46 & 0.30 & 2.27 & 7.67 & 23 & & & & 0.544 & 1. & & 7 & & 107 \\
\hline 76 & 7 & 1 & 0.09 & 43.49 & 0.26 & 2.23 & 7.53 & 23 & & & & & & & & & 127 \\
\hline 76 & 6 & 7 & 0.26 & 43.56 & 0. & 2.24 & 7. & 24 & & & & & & & & & 109 \\
\hline 76 & 7 & 1 & 0.26 & 43.66 & 0. & 2.27 & 7. & 24 & 4 & 3 & 0 & & & & & & 114 \\
\hline 576 & 6 & 7 & 0.44 & 43.74 & 0.3 & 2. & 7. & 24 & 8 & 3. & ) & 9 & 1.0 & & 6 & & 104 \\
\hline 576 & 7 & 1 & 0.36 & 43.76 & 0.3 & 2. & 7. & 24 & 5 & 3 & 0 & & 1. & & & 4 & 111 \\
\hline 576 & 7 & 1 & 0.46 & 43.86 & 0.32 & 2.24 & 7.65 & 24.0 & 9 & 3 & 2 & 5 & 1. & 1 & 5 & 66 & 117 \\
\hline 576 & 7 & 1 & 0.56 & 43.96 & 0.37 & 2.17 & 7.7 & 23.9 & 33 & 3 & 3 & 47 & 0.99 & 6.01 & 0.0 & 3 & 114 \\
\hline 576 & 7 & 1 & 0.66 & 44.06 & 0.42 & 2.12 & 7.7 & 23.9 & 0.268 & 3.2 & 0.72 & 0.671 & 0.92 & 5. & 0.0 & 2 & 108 \\
\hline 576 & 7 & 1 & 0.76 & 44.16 & 0.35 & 2.20 & 7.8 & 24.1 & 0.2 & 3.1 & 0.64 & 0.654 & 1.00 & 6. & 0.0 & & 116 \\
\hline 576 & 7 & 1 & 0.86 & 44.26 & 0.3 & 2.24 & 7.81 & 23.9 & 0.238 & 3.02 & 0.6 & 0.628 & 1.08 & 6.11 & 0.075 & 8 & 123 \\
\hline 576 & 7 & 1 & 0.96 & 44.36 & 0.28 & 2.26 & 7.7 & 23.7 & 0.249 & 3.00 & 0.66 & 601 & 1.07 & 9 & 4 & & 127 \\
\hline 576 & 7 & 1 & 1.06 & 44.46 & 0.32 & 2.31 & 7. & 24.1 & 0.265 & 3.01 & 0.67 & .626 & 1.04 & 5. & 0. & & 121 \\
\hline 576 & 7 & 1 & 1.16 & 44.56 & 0.32 & 2.33 & 7.8 & 24.1 & 0.285 & 3.02 & 0.7 & 9 & 1.06 & 6 & & 7 & 116 \\
\hline 576 & 7 & 1 & 1.26 & 44.66 & 0.28 & 2.32 & 7.8 & 24.1 & 0.287 & 3.00 & 0.7 & 0.615 & 1.13 & 6. & 0. & 6 & 117 \\
\hline 576 & 7 & 1 & 1.36 & 44.76 & 0.29 & 2.2 & 7.6 & 23.6 & 0.3 & 2.9 & 0.8 & 0.618 & 1.09 & 6. & $=$ & & 118 \\
\hline 576 & 7 & 1 & 1.46 & 44.86 & 0 & 2.21 & 7.7 & 23.8 & 0.2 & 3.13 & 0.7 & 0 & 1.03 & 6. & 1 & & 117 \\
\hline $576 \mathrm{~B}$ & 5 & 5 & 0.26 & 5.06 & 36 & 2.1 & 7.7 & 23.9 & 0.26 & 3.17 & 0. & 8 & 1.0 & & & & 109 \\
\hline $576 \mathrm{~B}$ & 5 & 5 & 0.46 & 45.26 & & 2. & 7. & 7 & & 3 & 0. & & 1. & & 5 & & 106 \\
\hline $576 \mathrm{~B}$ & 5 & 5 & 0.66 & 45.46 & 0. & 2. & & 23.6 & & 2.9 & & & 7 & & & & \\
\hline $576 \mathrm{~B}$ & 5 & 5 & 0.86 & .66 & 0. & 2.28 & 7. & 23.7 & & 2. & 0.7 & & 1.13 & & & & 107 \\
\hline 576 & 5 & 5 & 1.07 & .87 & 0 & 2. & 7. & 23.5 & & 2. & & & 1 & & & & 8 \\
\hline 576 & 5 & 5 & 1.26 & 6 & & 2. & 7.78 & 23.3 & 0. & 2.8 & 0. & & 1. & & & & 114 \\
\hline 76 & 5 & 5 & 1.46 & 26 & 0.35 & 2.16 & 7.77 & 23.6 & & 3. & 0.7 & & 1.07 & & & & 10 \\
\hline 576 & 5 & 6 & 0.06 & 36 & & 2. & 7. & 23.5 & & 3 & & & 3 & & & & 10 \\
\hline 576 & 7 & 2 & 0.56 & 6 & 0. & 2. & 7. & & & 2. & & & & & & & 15 \\
\hline 576 & 7 & 2 & 0.66 & 6 & 0 & 2. & 7.66 & 3 & & & & & 0 & & & & 16 \\
\hline 576 & 7 & 2 & 0 . & 4 & 0 & 2. & 7. & 23 & & & 0.83 & & 1 & & & & 23 \\
\hline 76 & 7 & 2 & 0.86 & 6 & 0 & 2. & & 23.3 & & 1 & 0.97 & & 1 & & 73 & & 21 \\
\hline 76 & 7 & 2 & 0.96 & 6 & 0. & 2. & 7. & 23 & & & & & 6 & & 22 & 3 & 119 \\
\hline & 7 & 2 & 1.08 & 8 & 0 & 2. & & & & & & & 3 & & 74 & 5 & 126 \\
\hline 76 & 7 & 2 & 1.16 & 7.06 & 0 & 2. & 7 & 22.9 & 0 & 2 & 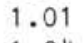 & 2 & 7 & & 74 & 7 & 128 \\
\hline 576 & 7 & 2 & 1.26 & 7.16 & 0 & 2. & 7 & 2 & 0 & 2 & 1 & 1 & 1.26 & & 4 & 16 & 125 \\
\hline 76 & 7 & 2 & 1.36 & 47.26 & 0. & 2. & 7. & & 0 & 2 & 1 & 2 & 9 & & 77 & & 130 \\
\hline & 7 & 2 & 1.46 & 47.36 & 0 . & 2.30 & 7 & 23 & 0. & 3 & 1. & 4 & 1.56 & 3 & 0.082 & 0. & 121 \\
\hline 76 & 7 & 3 & 0.05 & 47.45 & 0.2 & 2.3 & $7 \cdot 30$ & 23.2 & & & 1. & 7 & 1.43 & & 0.080 & 0.073 & 125 \\
\hline & 7 & 3 & 0.14 & .54 & 0 & 2 . & 7 & & & & & & 1. & & & 0.067 & 126 \\
\hline 78 & 7 & 3 & 0.26 & 47.66 & 0.28 & 2.24 & $7 \cdot 3$ & 23.1 & & & & & 1.3 & & & & 119 \\
\hline $57 t$ & 7 & 3 & 0.36 & 47.76 & 0.2 & 2.23 & 7. & 23.2 & & & 1.3 & & 1.35 & & & & 118 \\
\hline 7 & 7 & 3 & & & & & & & & & & & & & & & 119 \\
\hline & 7 & 3 & 0.56 & & 0.2 & 2.25 & & & & & 1.43 & 0.607 & 1.45 & 5. & 0.077 & 74 & 126 \\
\hline 57 & 7 & 3 & 0.66 & & & 2.19 & & 22.9 & & & & & & & 0.075 & & 126 \\
\hline & 7 & 3 & & & & & & & & & & & & & 79 & & 128 \\
\hline & 7 & 3 & 0.86 & & & & & & & & & & & & & & 127 \\
\hline 7 & 7 & 3 & .96 & 36 & 0.35 & 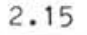 & 10 & 2.7 & 45 & 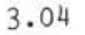 & 1 & 78 & 1.25 & & 0.076 & 0.084 & 124 \\
\hline
\end{tabular}




\begin{tabular}{|c|c|c|c|c|c|c|c|c|c|c|c|c|c|c|c|c|c|}
\hline 궁 & ัㅣㅇ & 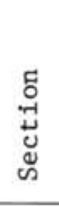 & 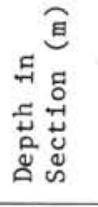 & 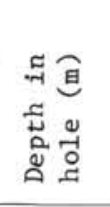 & $\mathrm{Na}$ & $\mathrm{Mg}$ & Al & Si & $P$ & K & $\mathrm{Ca}$ & $\mathrm{Ti}$ & Mn & $\mathrm{Fe}$ & $\mathrm{Ba}$ & S & $\mathrm{H}_{2} \mathrm{O}$ \\
\hline 76 & 7 & 3 & 1.06 & 48.46 & 0.37 & 2.10 & 7.14 & 22.6 & 0.720 & 3.02 & 1.77 & 0.605 & 1.17 & 6.03 & 0.074 & 0.086 & 126 \\
\hline 76 & 7 & 3 & 1.16 & 8.56 & 5 & 11 & $7 \cdot 37$ & 23.1 & & & 1.66 & 0.567 & 1.27 & 6.04 & .077 & 0.089 & 125 \\
\hline 576 & 7 & 3 & 1.26 & 48.66 & 0.39 & 2.12 & 7.42 & 22.9 & 0.695 & 3.03 & 1.73 & 0.538 & 1.30 & 6.02 & 0.077 & 0.090 & 123 \\
\hline 76 & 7 & 3 & 1.36 & 48.76 & 0.38 & 2.09 & 7.41 & 22.9 & 0.642 & 3.04 & 1.64 & 0.546 & 1.27 & 5.96 & 0.078 & 0.089 & 123 \\
\hline 576 & 7 & 3 & 1.46 & 48.86 & 0.39 & 2.08 & 7.40 & 22.9 & 0.705 & 3.05 & 1.70 & 0.555 & 1.17 & 6.00 & 0.074 & 0. & 121 \\
\hline 576 & 7 & 4 & 0.06 & 48.96 & 0.47 & 2.12 & 7.48 & 23.2 & 0.847 & 3. & 2.08 & 0. & 1.21 & 5.89 & 0.074 & 20 & 117 \\
\hline 576 & 7 & 4 & 0.16 & 49.06 & 0.40 & 2.07 & 7.24 & 22.8 & 0.809 & & 2.03 & 0 . & 1.22 & 5. & 0.075 & & 115 \\
\hline 576 & 7 & 4 & 0.26 & 49.16 & 0. & 2. & 7. & 22.5 & 0.789 & 3.07 & 2.03 & 0 & 1.26 & 5. & .075 & 0. & 121 \\
\hline 576 & 7 & 4 & 0.36 & 49.26 & 0.3 & 2.11 & 7. & 22.8 & 0.796 & 3.10 & 2.10 & 0.4 & 1.34 & 5.60 & 0.076 & 0. & 125 \\
\hline 576 & 7 & 4 & 0.46 & 49.36 & 0. & 2. & 7. & 22. & 36 & & 8 & 0 & 1.33 & & 0.074 & 32 & 127 \\
\hline 576 & 7 & 4 & 0.56 & 49.46 & 0.3 & 2 & 7. & 22 & 0 & 3. & 8 & 5 & 1.35 & 5 & 2 & 7 & 128 \\
\hline 576 & 7 & 4 & 0.66 & 49.56 & 0.35 & 2.14 & & 22 & 0 & 3. & 2. & 3 & 1. & & 0. & 0. & 125 \\
\hline 576 & 7 & 4 & 0.76 & 49.66 & 0.39 & 2.16 & 6.84 & 22 & & & 2 & & 1. & & 6 & & 125 \\
\hline 576 & 7 & 4 & 0.86 & 49.76 & 0.36 & 2.26 & 6.86 & 23.1 & 8 & & 2 & & & & 6 & 8 & 122 \\
\hline 576 & 7 & 4 & 0.96 & 49.86 & 0.34 & 2.18 & 7.05 & 22.8 & 0.845 & & 2.16 & & 1 & & 74 & 7 & 130 \\
\hline 576 & 7 & 4 & 1.06 & 49.96 & 0.34 & 2.16 & 7.07 & 22.9 & 0.832 & 3.29 & 2.15 & 0.4 & 1.55 & 5. & 0.078 & 0.096 & 125 \\
\hline 576 & 7 & 4 & 1.16 & 50.06 & 0.33 & 2.16 & 7.05 & 22.7 & 0.853 & 3.25 & 2.10 & 0.414 & 1.54 & 5.21 & 0.078 & 0.104 & 128 \\
\hline 576 & 7 & 4 & 1.26 & 50.16 & 0.40 & 2.16 & 7.10 & 22.9 & 0.779 & 3.26 & 2.03 & 0.415 & 1.54 & 5.25 & 0.077 & 0.081 & 129 \\
\hline 576 & 7 & 4 & 1.36 & 50.26 & 0.35 & 2.10 & 6.86 & 22.5 & 0.810 & $3 \cdot 36$ & 2.07 & 0.437 & 1.36 & 5.46 & 0.073 & 02 & 128 \\
\hline 576 & 7 & 4 & 1.46 & 50.36 & 0.35 & 2.12 & 6.89 & 22.9 & & 3.50 & 1.85 & 0.2 & 1.33 & 5.75 & 0.074 & 0. & 131 \\
\hline 576 & 7 & 5 & 0.06 & 50.46 & 0.31 & 2.11 & 7.12 & 22.7 & 0.8 & 3.35 & 2.16 & 0.379 & 1.51 & 4.94 & 0.072 & 0.100 & 131 \\
\hline 576 & 7 & 5 & 0.16 & 50.56 & 0.34 & 2.14 & 7.18 & 22.6 & 0. & 3.27 & 2.23 & & 1.51 & 4. & 0.073 & 0. & 125 \\
\hline 576 & 7 & 5 & 0.26 & 50.66 & 0.33 & 2.14 & 7.27 & 23.0 & 0. & 3.30 & 1.95 & 0. & 1.38 & & 0. & 0 & 118 \\
\hline 576 & 7 & 5 & 0.36 & 50.76 & 0.33 & 2.15 & 7. & 22.9 & 0. & $3 \cdot 31$ & 2.02 & 0.2 & 1.34 & 5. & 0.075 & 0.102 & 116 \\
\hline 576 & 7 & 5 & 0.46 & 50.86 & 0.33 & 2.11 & 7. & 22.9 & & & 1.97 & 0. & 1.35 & & 0. & & 119 \\
\hline 576 & 7 & 5 & 0.56 & 50.96 & 0.33 & 2.13 & 7. & 23.0 & 0. & 3. & 2.11 & 8 & 1. & & 0. & 6 & 117 \\
\hline 576 & 7 & 5 & 0.66 & 51.06 & 0. & 2.14 & 7. & 23 & 0 & & 2.12 & & 1. & & 0. & 5 & 118 \\
\hline 576 & 7 & 5 & 0.76 & 51. & 0 & 2.12 & 7. & 2 & 6 & & 2. & & 1. & & 0. & 5 & 120 \\
\hline 576 & 7 & 5 & 0.86 & 51.26 & 0 & 2. & 7 & 5 & 8 & & 2. & 6 & 1 & & 5 & 2 & 116 \\
\hline 576 & 7 & 5 & 0.96 & 51.36 & 0. & 2. & 7 & 2 & 3 & & 2.56 & & 1 & & 6 & 4 & 116 \\
\hline 576 & 7 & 5 & 1.06 & 51.46 & 0.36 & 2.06 & 7 & & 0 & & 2. & & 8 & & 4 & 1 & 118 \\
\hline 576 & 7 & 5 & 1.16 & 51.56 & 0.38 & 2.06 & 7 & 7 & & & 2 & 0 & 1 & 7 & 74 & 0.122 & 119 \\
\hline 576 & 7 & 5 & 1.26 & 51.66 & 0.38 & 1.98 & 6 & 2 & 8 & & 6 & 2 & 2 & 2 & 74 & 0.126 & 116 \\
\hline 576 & 7 & 5 & 1.36 & 51.76 & 0.36 & 2.07 & 7 & 9 & & & 2. & 8 & 1 & 9 & 77 & 01 & 118 \\
\hline 576 & 7 & 5 & 1.46 & 51.86 & 0.38 & 2.07 & 7 & 4 & 0 & & & & & & 0.077 & 14 & 117 \\
\hline 576 & 7 & 6 & 0.06 & 51.96 & 0.37 & 2.09 & 0 & 4 & 9 & & 2.32 & 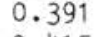 & 1.27 & 5 & 0.074 & 0.112 & 115 \\
\hline 576 & 7 & 6 & 0.16 & 52.06 & 0.35 & 2.16 & 7 & 22.8 & 0.786 & 0 & 2.04 & 0. & 1.28 & 5.38 & 0.077 & 0.096 & 111 \\
\hline 576 & 7 & 6 & 0.26 & 52.16 & 0.34 & 2.17 & 7.22 & 23.0 & 0.672 & 3.23 & 1.78 & 0. & 1.30 & 8 & 0.080 & 0.098 & 112 \\
\hline 576 & 7 & 6 & 0.36 & 52.26 & 0.34 & 2.19 & 7.31 & 23.3 & 0.666 & 3.2 & 1.79 & 0.42 & 1.38 & 7 & 0.078 & 0.089 & 116 \\
\hline 576 & 7 & 6 & 0.46 & 52.36 & 0.34 & 2.19 & $7 \cdot 32$ & 23.2 & 0.7 & 3.28 & 1.99 & 0. & 1.37 & & 0.079 & 0.092 & 112 \\
\hline 576 & 7 & 6 & 0.56 & 52.46 & 0.34 & 2.15 & 7.22 & 22.9 & 0.8 & 26 & 2.08 & 0.39 & 1.32 & 5.25 & 0.076 & 0.097 & 114 \\
\hline 576 & 7 & 6 & 0.66 & 52.56 & 0.35 & 2.17 & 7.19 & 22.9 & 0.838 & 3 & 2.12 & 0.3 & 1.28 & 5.17 & 0.075 & 0.107 & 110 \\
\hline 576 & 7 & 6 & 0.76 & 52.66 & 0.38 & 2.16 & 7.17 & 22.8 & 0.898 & & 2.20 & 0.3 & 1.28 & & 0.075 & 0.115 & 111 \\
\hline 576 & 7 & 6 & 0.86 & 52.76 & 0.35 & 2.17 & 7.22 & 22.9 & 0.731 & & 1.95 & 0.390 & 1.31 & 27 & 0.078 & 91 & 112 \\
\hline 576 & 7 & 6 & 0.96 & 52.86 & 0.35 & 2.19 & 7.23 & 22.9 & 0.734 & 24 & 1.84 & 0.393 & 1.29 & 5.33 & 0.076 & 98 & 111 \\
\hline 576 & 7 & 6 & 1.06 & 52.96 & 0.33 & 2.22 & 7.39 & 23.4 & 0.656 & 3.33 & 1.74 & 0.403 & 1.35 & $5 \cdot 38$ & 0.078 & 0 . & 112 \\
\hline 576 & 7 & 6 & 1.16 & 53.06 & 0.36 & 2.18 & 7.27 & 23.1 & 0.612 & & 1.62 & 0.390 & 1.24 & & 0.073 & & 110 \\
\hline 576 & 7 & 6 & 1.26 & 53.16 & 0.35 & 2.18 & 7.45 & 23.6 & 0.520 & & 1.37 & 0.403 & 1.21 & 5.48 & 0.075 & 0.076 & 109 \\
\hline 576 & 7 & 6 & 1.36 & 53.26 & 0.38 & 2.16 & 7.49 & 23.5 & 0.6 & & 1.55 & 0. & 1.21 & & 0.074 & & 114 \\
\hline 576 & 7 & 6 & 1.46 & $\cdot 36$ & 0.35 & 2.14 & 7.39 & 23.4 & 0.558 & & 1.44 & 0.3 & 1.26 & & 0.077 & 0. & 111 \\
\hline 576 & 7 & 7 & 0.06 & & 0.36 & 2.17 & 7.48 & 23.7 & 0.345 & & 1.00 & 1 & 1.27 & & 0.074 & 71 & 109 \\
\hline 57 & 7 & 7 & 16 & & & 2.17 & & 24.1 & & & & & 1.31 & & 0.073 & & 110 \\
\hline 57 & 7 & 7 & 0.26 & & & 2.17 & 9 & 23.7 & & & & 4 & 1.35 & & 0.076 & & 109 \\
\hline 576 & 7 & 7 & 0.36 & & 0.37 & 2.18 & 7.51 & 23.8 & 0.337 & & 0.96 & 0. & 1.36 & & 0.074 & 0. & 110 \\
\hline & 7 & 7 & 0.46 & & & 2.2 & 7.5 & 24.2 & & & & & 1.4 & & 0.078 & & 113 \\
\hline & 8 & 1 & 0.26 & & & .18 & 7. & 23.5 & & & .06 & 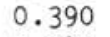 & 1.42 & 5. & 0.077 & 0.076 & 119 \\
\hline 57 & 8 & 1 & 0.36 & & & .19 & 7.36 & 23.5 & & & 1.20 & 0. & 1.35 & 5.19 & 0.076 & 0.081 & 120 \\
\hline & 8 & 1 & 0.46 & & & & & 23.3 & & & & & 1.71 & & 0.082 & 0.084 & 128 \\
\hline & 8 & 1 & 0.56 & & & 12 & & & & & 1.60 & 0.397 & 1.71 & 5.09 & 0.080 & 0.086 & 123 \\
\hline 19. & 8 & 1 & 0.66 & 54.76 & & 2.0 & . & 22. & & & 1. & & 1.81 & & 0.077 & & 124 \\
\hline 576 & 8 & 1 & 0.76 & & נד. & 2.09 & 7.08 & 23.1 & 0.653 & 9.45 & 1.81 & 0.397 & 1.95 & 5.11 & 0.083 & 0.084 & 124 \\
\hline
\end{tabular}


Table 5. (Continued).

\begin{tabular}{|c|c|c|c|c|c|c|c|c|c|c|c|c|c|c|c|c|c|}
\hline $\begin{array}{l}\text { 검 } \\
\text { 오 }\end{array}$ & $\stackrel{\stackrel{ٌ}{0}}{0}$ & 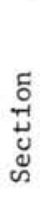 & 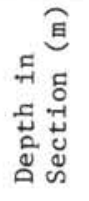 & 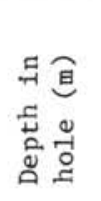 & $\mathrm{Na}$ & $\mathrm{Mg}$ & Al & Si & $\mathrm{P}$ & K & $\mathrm{Ca}$ & $\mathrm{Ti}$ & $\mathrm{Mn}$ & $\mathrm{Fe}$ & $\mathrm{Ba}$ & S & $\mathrm{H}_{2} \mathrm{O}$ \\
\hline $576 \mathrm{~B}$ & 6 & 4 & 0.46 & 54.92 & 0.32 & 5 & 0 & 2 & 0.473 & 3.49 & 1.35 & 02 & 1.67 & 5.40 & 0.079 & 92 & 118 \\
\hline 576 & 8 & 1 & 0.86 & 54.96 & 0 & 2 & 6 & 0 & & 3 & & 9 & 8 & 8 & & & 128 \\
\hline 76 & 8 & 1 & 0.96 & 55.06 & 0 & 2. & & 22.5 & & & & 0 & 3 & 3 & 0 & & 121 \\
\hline $576 \mathrm{~B}$ & 6 & 4 & 0.66 & 55 & 0. & 2. & & 23.0 & & & & 5 & & & & & 21 \\
\hline 576 & 8 & 1 & 1.06 & 55 & 0. & 2 & 6. & 22 & 33 & 3. & & 9 & & 3 & 0 & & 128 \\
\hline 576 & 8 & 1 & & 55 & 0. & & & 22 & & & 2 & & 2 & & & 9 & 15 \\
\hline $576 \mathrm{~B}$ & 6 & 4 & 0 & 55 & 0. & 2 & 6 & 2 & & & & 9 & & 3 & 7 & 3 & 14 \\
\hline 576 & 8 & 1 & 1. & 55 & 0. & 2. & 6 & 22 & & 3 & 4 & 3 & 0 & & & & 123 \\
\hline $576 \mathrm{~B}$ & 6 & 4 & 1 & 55 & 0. & 2. & 6 & 2 & 0 & 3 & 2 & 5 & 3 & & 4 & 6 & 107 \\
\hline $576 \mathrm{~A}$ & 6 & 5 & 1 & $5=$ & 0. & 2. & 6 & 2 & 3 & 3 & 1 & 8 & 0 & 1 & & .077 & 95 \\
\hline 576 & 8 & 1 & 1. & 5 & 0 & 2 & 6 & 22 & 1 & 3 & 2 & 7 & 0 & 0 & 081 & .081 & 116 \\
\hline 576 & 8 & 2 & 0.05 & 55.65 & 0.32 & 2.28 & 6.56 & 22.7 & 0.508 & 3.32 & 2.06 & 0.394 & 1.62 & 5.87 & 0.079 & 0.063 & 118 \\
\hline $576 \mathrm{~B}$ & 6 & 4 & 1.46 & 55.92 & 0.29 & 2.29 & 6.74 & 22.9 & 0.426 & 3.41 & 1.56 & 0.387 & 1.91 & 6.25 & 0.084 & 0.088 & 115 \\
\hline 576 & 8 & 2 & 0.67 & 56.27 & 0.26 & 2.17 & 6.67 & 22.6 & 0.446 & 3.35 & 2.42 & 0.386 & 1.58 & 5.53 & 0.080 & 0.071 & 121 \\
\hline 76 & 8 & 2 & 0.78 & & 0.29 & 2.14 & 6.29 & 21.2 & 0.348 & 3.11 & 3.60 & 0.353 & 1.75 & 6.42 & 0.083 & 0.058 & 122 \\
\hline $76 \mathrm{~B}$ & 6 & 5 & 0.55 & & 0.29 & 2.30 & 6.86 & 23.2 & 0.348 & 3.36 & 0.99 & 0.374 & 1.86 & 6.61 & & & 126 \\
\hline 576 & 8 & 2 & 1.06 & & 0.29 & 2.18 & 6.86 & 23.0 & 0.419 & 3.37 & 1.64 & 0.371 & 1.54 & 5.62 & 0.078 & 0. & 123 \\
\hline 76 & 8 & 2 & 1.16 & & 0.29 & 2.24 & 7.11 & 23.8 & 0.359 & 3.42 & 0. & 0.390 & 1. & & & & 122 \\
\hline 76 & 6 & 5 & 0.86 & & 0.3 & 2.18 & 6.94 & 23.2 & 0.398 & 3.43 & 1.60 & 0.379 & 1. & 5. & 0. & & 113 \\
\hline 576 & 8 & 2 & 1.26 & & 0.2 & 2.20 & 6.95 & 23.3 & 0.3 & 3. & 1. & 0. & 1. & & & & 11 \\
\hline 6 & 8 & 2 & 1.36 & & 0.2 & 2.23 & 6.93 & 23.5 & 0.3 & 3. & 0. & 0.376 & 1. & & & & 120 \\
\hline $576 \mathrm{~B}$ & 6 & 5 & 1.06 & & 0. & 2.17 & 7. & 23.4 & 0 & 3 . & 1. & 9 & 1. & & & & 118 \\
\hline 576 & 8 & 2 & 1.46 & & 0. & 2.23 & 6. & 21.9 & 0 & 3. & 3. & 6 & 1. & & & & 120 \\
\hline 576 & 8 & 3 & 0.04 & & & & & 23 & & & & & & & & & 30 \\
\hline 5 & 6 & 5 & 1. & & & 2. & & 2. & & & & & & & & & 119 \\
\hline $576 \mathrm{~A}$ & 6 & 7 & 0. & 57 & 0. & 2. & & & & & & & & & & & 110 \\
\hline $576 \mathrm{~A}$ & 6 & 7 & 0. & & 0. & 2. & & 2. & & & 3 & & & & 1 & 5 & \\
\hline 576 & 8 & 3 & 0 & & 0 & 2 & 6 & 2 & & & 4 & 5 & 6 & 9 & 0 & 4 & 126 \\
\hline $576 \mathrm{~B}$ & 6 & 6 & 0.2 & & 0 & 2 & 6 & 2 & 1 & & 6 & 5 & 1 & 7 & 1 & 76 & 125 \\
\hline 576 & 8 & 3 & 0.92 & 2 & 0.2 & 2.3 & 7.0 & 24.1 & 0.2 & 3 . & 0 . & 0. & 1. & 5. & 0.076 & 0.062 & 117 \\
\hline 576 & 8 & 3 & 1.01 & 11 & 0.27 & 2.40 & 6.48 & 22.9 & 39 & 3.10 & 0.9 & 0.351 & 1.61 & 7.81 & 0.091 & 0. & 133 \\
\hline $76 \mathrm{~B}$ & 6 & 6 & 0.76 & 22 & 0.24 & 2.31 & 6.87 & 23.5 & 0.393 & 3. & 1. & 1 & 1. & 6.45 & 0.086 & 0. & 121 \\
\hline 6 & 8 & 3 & 1.47 & 57 & 0.30 & 2.31 & 7.02 & 24.2 & 0.380 & 3. & 1.01 & 0.416 & 1. & $5.77^{\circ}$ & 0.074 & 0. & 116 \\
\hline 76 & 8 & 4 & 0.03 & 63 & 0.27 & 2.32 & 6.91 & 23.7 & 0.39 & 3. & 1.0 & .392 & 1. & 5 & 0.083 & 0 . & 112 \\
\hline 76 & 8 & 4 & 0.13 & 58.73 & 0.3 & 2.35 & 6.89 & 23.6 & $.5^{5}$ & 3. & 1.43 & 0.391 & 0. & 6. & 0. & 0. & 108 \\
\hline $6 \mathrm{~B}$ & 6 & 6 & 1.36 & 82 & 0.2 & 2.33 & 6.90 & 23.7 & 0.37 & 3. & 1.0 & 0. & 1. & 5 & 0. & 0. & 115 \\
\hline 576 & 8 & 4 & 0.23 & 33 & 0.28 & 2.4 & 6.28 & 23.0 & 0.43 & 3.13 & 1.9 & 8 & 1. & 6. & 0. & 2 & 124 \\
\hline 576 & 8 & 6 & 0.77 & 37 & 0.30 & 2.38 & 6.92 & 24.0 & 0.4 & 3. & 1.0 & ? & 1. & 5 & 0 . & 5 & 113 \\
\hline $576 \mathrm{~B}$ & 7 & 3 & 1.45 & 45 & $0.2^{7}$ & 2.47 & 6. & 24.4 & 0 & 3. & 0. & 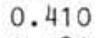 & 1. & 5 & 0. & & 109 \\
\hline 576 & 8 & 6 & 0.90 & 50 & 0.2 & 2.4 & 6.8 & 24.4 & 0.2 & 3. & 0 . & 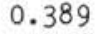 & 1. & & 0 & & 118 \\
\hline 576B & 7 & 4 & 0.91 & 41 & 0.1 & 2.5 & 6.8 & 24.3 & 0.3 & 3 & 0. & 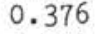 & 1 & & & & 1 \\
\hline 576 & 8 & 7 & 0.39 & 9 & 0.2 & 2.5 & 6 & 24.7 & 0.33 & 2 & 0.81 & & & & & & 21 \\
\hline $5^{5}$ & 8 & C & 0 & & 0 & 2.56 & & 24.7 & & & & & & & & & 124 \\
\hline $576 \mathrm{~B}$ & 7 & 4 & 1.28 & & 0 & 2. & 6 & 24.1 & 0 & 3 & 1 & & & & 0. & & 111 \\
\hline B & 7 & 5 & 0.79 & & 0 & 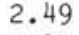 & & 6 & & & & & 1 & & & & 110 \\
\hline $576 \mathrm{~B}$ & 7 & 6 & 0.46 & & 0.07 & 2.84 & & 24.8 & & & & & & & & & 138 \\
\hline $576 \mathrm{~B}$ & 7 & 6 & 0.89 & & 0.06 & 2.86 & 6.61 & 25.3 & & 3. & 0. & 0 . & 0.76 & & & & 133 \\
\hline $576 \mathrm{~B}$ & 7 & 7 & 0.30 & & 0.06 & 2.9 & 6. & 24.9 & & & & & & & 0. & & 139 \\
\hline & 8 & 2 & 0.81 & & $0.2^{\prime}$ & 2.3 & 6.51 & 24.0 & & 3. & 2.09 & 0.385 & 1.0 & 4. & 0.090 & 0. & 102 \\
\hline $576 \mathrm{~B}$ & 8 & 3 & 0.29 & 70.74 & 0.12 & 2.76 & 6.61 & 25.1 & 0.2 & 3.2 & 0.70 & 0. & 0. & 4.86 & 0.096 & 0.044 & 126 \\
\hline & 8 & 3 & 0.65 & 71.10 & 0.16 & 2.61 & 6.60 & 24.8 & 0.5 & & 1.17 & 0.3 & 1. & 4.88 & 0.093 & 0.070 & 117 \\
\hline $576 \mathrm{~B}$ & 8 & 3 & 0.96 & 71.41 & 0.19 & 2.57 & 6.18 & 23.5 & 0.3 & & 2.66 & & 0. & 4.88 & 0.087 & 0.057 & 116 \\
\hline & 8 & 4 & 0.21 & 72.16 & 0.2 & 2.65 & 6.52 & 24.7 & 0.46 & 3. & 1.20 & 0.388 & 1.2 & 5. & 0.087 & 0.059 & 108 \\
\hline $576 \mathrm{~B}$ & 8 & 4 & 0.41 & 72.36 & 0.38 & 2.50 & 6.24 & 23.7 & 0.47 & 3. & 1.33 & & 1.5 & & 0.094 & 0.075 & 103 \\
\hline & 8 & 4 & 0.99 & 72.94 & 0.37 & 2.4 & 6.00 & 23.4 & 0.5 & 3. & 2.41 & & 1. & 6. & 0.093 & 0.070 & 100 \\
\hline $576 \mathrm{~B}$ & 8 & 5 & 0.58 & 74.03 & 0.24 & 2.7 & 6. & 25.6 & 0.3 & 3. & 0.84 & 0.4 & 0. & & 0.083 & 0.057 & 109 \\
\hline & 8 & 5 & 1.42 & 74.87 & 0 . & & & 25.5 & & & & & & & 0.084 & 0.056 & 113 \\
\hline & 8 & 6 & 0.44 & 75.39 & 0.19 & .03 & ك & 25.3 & 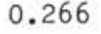 & & 1.10 & 0.397 & 0.82 & 5.18 & 0.086 & 0.052 & 112 \\
\hline
\end{tabular}


Table 6. Elemental abundance (wt. \%) and water content (\% dry wt.) of Hole 578 sediment samples.

\begin{tabular}{|c|c|c|c|c|c|c|c|c|c|c|c|c|c|c|c|c|c|}
\hline Lab ID & แัّ & 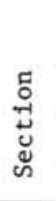 & 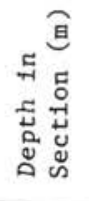 & 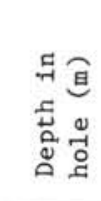 & $\mathrm{Na}$ & $\mathrm{Mg}$ & Al & S1 & $P$ & K & $\mathrm{Ca}$ & T1 & $\mathrm{Mn}$ & $\mathrm{Fe}$ & $\mathrm{Ba}$ & S & $\mathrm{H}_{2} \mathrm{O}$ \\
\hline 7319 & 1 & 1 & 16 & 6 & 6 & .51 & 2 & .2 & 068 & 95 & 0.86 & 360 & 9 & .28 & 107 & 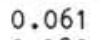 & or \\
\hline & 1 & 1 & (3) & & & 57 & & .6 & 67 & & 97 & & & & & & 51 \\
\hline 323 & 1 & 1 & 86 & 86 & 31 & 1.76 & 92 & 27.1 & 053 & .23 & 5 & .370 & 4 & .99. & 94 & & 206 \\
\hline 324 & 1 & 1 & 1.06 & & 0.22 & 1.78 & & & 53 & & & & & & & & 215 \\
\hline 7325 & 1 & 1 & 26 & & & & & & & & & & & & & & 188 \\
\hline 326 & 1 & 1 & & & & & & & & & & & & & & & 75 \\
\hline 7327 & 1 & 2 & 0.06 & 1.56 & & 1.84 & 1. & 26.7 & .053 & & & 35 & & & & & 170 \\
\hline 329 & 1 & 2 & 0.50 & 2.00 & 0 & 1.73 & 6. & 27.0 & .053 & 8 & 0.78 & & 1 & & & & 166 \\
\hline & 1 & 2 & 1.26 & & 0.84 & 0.95 & 5.92 & 27.9 & .043 & & & & & & & & 105 \\
\hline & 1 & 2 & 1.46 & & & & & & & & & & & & & & \\
\hline & 1 & 3 & 0 & & & & & & & & & & & & & & 67 \\
\hline 6 & 1 & 3 & 0 & & & & & & & & & & & & & & 58 \\
\hline 337 & 1 & 3 & 0.46 & 3.40 & 0.51 & 1.77 & 7.12 & 26.8 & .056 & 25 & 0.98 & 1 & 1 & 4. & 0 & & 133 \\
\hline & 1 & 3 & 0.66 & 3.66 & 0.44 & 1.72 & 7.03 & 26.7 & .056 & 0 & 0. & 1 & 0 & & & & 156 \\
\hline & 1 & 3 & 0.86 & 3.86 & 0.51 & 1.82 & & 26.5 & 0 & & & & & & & & 6 \\
\hline & 2 & 1 & 0.06 & & & & & & & & & & & & & & \\
\hline & 2 & 1 & & & & & & & & & & & & & & & 83 \\
\hline 7347 & 2 & 1 & 0.86 & & & & & & & & & & & 9 & & & 171 \\
\hline & 2 & 1 & 1.26 & 6.06 & 0.31 & 1.77 & 6.96 & 27.3 & .050 & • 39 & .66 & 92 & .20 & 4.10 & & & 166 \\
\hline & 2 & 2 & 0.46 & 6.76 & 0.24 & 1.68 & .69 & 27.4 & 9 & .19 & 0. & & 4 & & & & 4 \\
\hline & 2 & 2 & 0.86 & 7.16 & 0.33 & 1.73 & & 27.0 & & 7 & & & & & & & 173 \\
\hline & 2 & 2 & 1.26 & & & & & & & & & & & & & & \\
\hline & 2 & 3 & 1 & & & & & & & & & & & & & & 72 \\
\hline 61 & 2 & 3 & 66 & 8.4 & 0.61 & 1.37 & 0 & $7 \cdot 3$ & 9 & 3 & 5 & 6 & 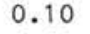 & 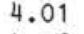 & & & 152 \\
\hline & 2 & 3 & 0.86 & 8.66 & 0.41 & 1.79 & 7.04 & 26.9 & .0 & .0 & 0.93 & 0.2 & . & 4. & & & 160 \\
\hline & 2 & 3 & 1.06 & 8.8 & 0.52 & $16 ?$ & & 27 & & & & & & & & & \\
\hline & 2 & 3 & & & & & & 2 & & & & & & & & & \\
\hline & 2 & 4 & 0 & & & & & & & & & & & & & & 48 \\
\hline & 2 & 4 & 64 & 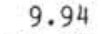 & .49 & .08 & 1 & 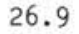 & & & & & & & & & 151 \\
\hline & 2 & 4 & .06 & 6 & .50 & .64 & 8 & .3 & & 8 & 0. & & 0 & 4. & & & 4 \\
\hline & 2 & 4 & 1.46 & .76 & 0.40 & 1.73 & 6.97 & 7.0 & .050 & . 3 & 0.8 & 2 & 8 & & & & 45 \\
\hline & 2 & 5 & 26 & 6 & .3 & 7 & 6 & 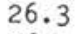 & .0 & 2.3 & 0. & & & & & & 51 \\
\hline & 2 & 5 & 66 & & & 1. & & 2 & & & & & & & & & \\
\hline & 2 & 5 & & & & & & & & & & & & & & & \\
\hline & 2 & 6 & 06 & & & & & & & & & & & & & & 153 \\
\hline 3 & 3 & 1 & 0.04 & 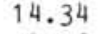 & 0.84 & 1.47 & 6 & 27.0 & 0.054 & 1.69 & 1 & & 3 & 4. & & & 119 \\
\hline & 3 & 1 & 0.46 & .7 & 0.4 & 1.77 & 6. & 26.9 & .0 &. & 0. & & 4 & 4. & & & 139 \\
\hline & 3 & 1 & 0.86 & & .3 & 1.8 & 7 & 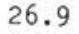 & & & & & & & & & \\
\hline & 3 & 2 & & & & & & & & & & & & & & & 6 \\
\hline & 3 & 2 & 0.46 & & & & & & & & & & & & & & 52 \\
\hline & 3 & 2 & 0.86 & 66 & 0.54 & 1.76 & 6.63 & 26.0 & 1 & 2. & 1. & & 3 & 3. & & & 150 \\
\hline & 3 & 2 & .26 & 06 & 0.42 & 1.62 & 6.83 & 27.0 & 0.0 & 2. & 1. & 5 & 0.09 & 4. & & & 160 \\
\hline & 3 & 2 & .46 & .26 & 0.4 & 1.70 & 7.09 & 26.8 & 0.0 & 2.2 & 0 & 8 & 0.0 & & & & 141 \\
\hline & 3 & 3 & .06 & & .4 & 1. & 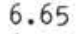 & 27.1 & & & & & & & & & 0 \\
\hline & 3 & 3 & & & & & & 3 & & & & & & & & & \\
\hline & 3 & 3 & & & & & & & & & & & & & & & 136 \\
\hline & 3 & 3 & 0.83 & & 0.45 & 1.51 & 6. & 27.0 & 0 & 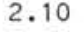 & 0 & & .10 & 4. & & & 159 \\
\hline 5 & 3 & 3 & 1.26 & 6 & 0.5 & 1.23 & 6. & 27.6 & 0.0 & 2. & 0. & 0 & 0.09 & 3. & & & 137 \\
\hline D. & 3 & 4 & 0 & 86 & 0.5 & 1.38 & 6. & 27.5 & 0.0 & 2. & 0. & 5 & 0. & 3. & & & 149 \\
\hline & 3 & 4 & 4 & & .4 & 1.77 & 7. & 26. & 0.052 & 2. & & & & & & & 5 \\
\hline & 3 & 4 & & & 0 & 61 & 7 & 1 & 0.0 & 2 & 6 & & 0 . & & & & \\
\hline & 3 & 4 & & & & & & & & & & & & & & & \\
\hline & 3 & 5 & & & & & & .8 & & 2 . & & & & 3 & & & 120 \\
\hline 7417 & 3 & 5 & .462 & 76 & 0.58 & 1.64 & 7.03 & 26.9 & 0.051 & 2. & 0. & & 0. & 4. & & 5 & 122 \\
\hline 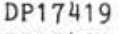 & 3 & 5 & 8 & 1 & 0.5 & 1 & 6.5 & 27.1 & .051 & 2.1 & 1. & 8 & 1 & & & & 7 \\
\hline & 3 & 5 & & & & & & & & & & & & & & & \\
\hline & 3 & 6 & 06 & & & & & 27.3 & & & & & & & & & 118 \\
\hline & 3 & 6 & & & & 1. & 7. & 27.0 & & 2.38 & & & 0.09 & & & .063 & 156 \\
\hline & 3 & 6 & & & & & & 27.2 & & 2.54 & & & & & & & 130 \\
\hline DP17429 & 3 & 7 & 06 & & 0.34 & & 7.00 & 27.0 & 0.050 & 2.33 & 0.78 & & 0.18 & & & & 151 \\
\hline 1743 & 3 & 7 & & & 0.3 & & & 26.9 & 0.047 & 2.37 & 0.72 & 0.4 & 0.13 & 4. & & & 162 \\
\hline & 4 & 1 & 0.26 & & & & & 27.1 & & & & & & & & & 152 \\
\hline & 4 & 1 & & .46 & 0.3 & 1.76 & & 27.0 & 0.049 & 2.35 & 0.81 & 0.1 & 0.15 & & & & 149 \\
\hline DP17437 & 4 & 1 & 1.13 & 24.93 & 0.32 & 1.73 & 6.96 & 26.8 & 0.049 & 2.32 & 0.77 & 0.394 & 0.13 & 4.33 & 0.086 & 0.071 & 160 \\
\hline
\end{tabular}




\begin{tabular}{|c|c|c|c|c|c|c|c|c|c|c|c|c|c|c|c|c|c|}
\hline Lab ID & بัّ & 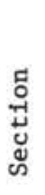 & 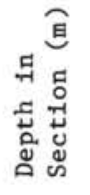 & 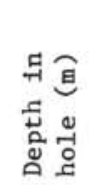 & $\mathrm{Na}$ & $\mathrm{Mg}$ & Al & Si & $\mathrm{P}$ & K & $\mathrm{Ca}$ & Ti & Mn & $\mathrm{Fe}$ & $\mathrm{Ba}$ & S & $\mathrm{H}_{2} \mathrm{O}$ \\
\hline DP17439 & 4 & 1 & 1.45 & .25 & 0.29 & 1.84 & 7.01 & 26.8 & 0.049 & 2.42 & 0.71 & 0.399 & 0.20 & 4.44 & 0.091 & 0.086 & 160 \\
\hline DP1 7443 & 4 & 2 & 0.66 & .96 & 0.45 & 1.80 & 7.12 & 26.9 & 0.048 & 2.35 & 0.79 & 399 & 0.11 & 4.62 & 0.090 & & 135 \\
\hline DP17445 & 4 & 2 & 1.08 & 38 & 0.58 & 1.69 & 7.11 & 26.7 & 0.051 & 2.21 & 1.00 & 0.409 & 0.11 & 4.30 & 0.082 & 0.075 & 121 \\
\hline DP17447 & 4 & 2 & 1.46 & 26.76 & 0.51 & 1.59 & 7.07 & 27.0 & 0.048 & 2.20 & 0.91 & 0.385 & 0.09 & 4.20 & 0.091 & & 133 \\
\hline DP17457 & 4 & 3 & 0.26 & 27.06 & 0.57 & & 6. & 27.0 & 50 & & & 90 & & & & 55 & 119 \\
\hline DP17459 & 4 & 3 & 0.66 & 27.46 & 0.44 & 1.68 & 7.08 & 27.4 & 0.048 & 2.25 & 0.86 & 0.390 & 0.11 & 4.43 & 0.093 & .074 & 154 \\
\hline DP17463 & 4 & 3 & 1.46 & 28.26 & 0.56 & 1.64 & 6.71 & 27.2 & 0.051 & 2.25 & 0.77 & 0.358 & 0.11 & 4.70 & 0.092 & 0.058 & 134 \\
\hline DP17465 & 4 & 5 & 0.32 & 30.12 & 0.40 & 1.90 & 7.09 & 26.3 & 0.048 & 2.53 & 0.57 & 0.395 & 0.12 & 4.48 & & 79 & 134 \\
\hline DP17467 & 4 & 5 & 0.66 & 30.46 & 0.47 & 1.80 & 7.23 & 26.6 & 0.049 & & 0.76 & 0.400 & 0.09 & 4.32 & 0.090 & 0.067 & 127 \\
\hline DP17469 & 4 & 5 & 1.06 & 30.86 & 0.48 & 1.62 & 6.77 & 26.8 & 0.048 & 2.18 & 0.89 & 0.361 & 0.10 & & & & 147 \\
\hline DP17471 & 4 & 5 & 1.46 & 31.26 & 0.55 & 1 & & 27.0 & 4 & & & 3 & & & & & 131 \\
\hline DP17473 & 4 & 6 & 0.26 & 31.56 & 0.54 & 1.65 & 6.98 & 27.0 & 0.053 & 2.26 & 0.79 & 0.371 & 0.16 & 4.42 & 0.086 & 0.055 & 130 \\
\hline DP17474 & 4 & 6 & 0.46 & 31.76 & 0.51 & 1.68 & 7.08 & 26.9 & 0.053 & 2.41 & 0.76 & 0.374 & 0.09 & 4.27 & 0.089 & 0.057 & 126 \\
\hline DP17477 & 4 & 6 & 1.14 & 32.44 & 0.52 & 1.72 & 7.05 & 26.8 & 0.055 & 2.35 & 0.81 & & & 4.73 & & & 129 \\
\hline DP1 & 5 & 1 & 0.47 & 33.77 & 0.49 & 1.77 & & 27.2 & 0.052 & & 9 & & 0.44 & 4.50 & & 53 & 130 \\
\hline DP17481 & 5 & 1 & 1.26 & 34.56 & 0.52 & 1. & 6. & 27.6 & 0.050 & 4 & & 0 & 0 & & 6 & & 143 \\
\hline DP17483 & 5 & 2 & 0.06 & 34.86 & 0.56 & 1.65 & 6.93 & 26.7 & 0.055 & 2.37 & 0.74 & 0.374 & 0.12 & 4.49 & 0.085 & 0.058 & 123 \\
\hline DP17485 & 5 & 2 & 0.51 & 35.31 & 0.57 & 1.58 & 6.88 & 26.9 & 0.055 & 2.18 & 1.02 & 0.379 & 0.10 & 4.34 & 0.085 & 0. & 133 \\
\hline DP17487 & 5 & 2 & 0.85 & & 0.57 & 1.79 & 7.06 & 27.0 & 0.058 & 2.45 & 0.74 & 0.416 & 0.15 & 4.73 & 0 . & & 108 \\
\hline DP17489 & 5 & 2 & 1.26 & 36.06 & 0.46 & 1.77 & 7.19 & 26.7 & 0.0 & & 0.69 & & & & & & 129 \\
\hline DP17491 & 5 & 3 & 0.06 & 36.36 & 0.46 & 1. & 6. & 27.4 & 0.0 & & & & & & & & 142 \\
\hline DP17493 & 5 & 3 & 0.45 & 36.75 & 0.56 & 1.49 & 6. & 27.3 & 3 & & & & & 4 & 6 & 9 & 163 \\
\hline DP17495 & 5 & 3 & 0.86 & 37.16 & 0.55 & 1.60 & 7.01 & 27.1 & 0.053 & 2.22 & 0.91 & 0.387 & 0.09 & 4.44 & 0.094 & 62 & 131 \\
\hline DP17499 & 5 & 4 & 0.06 & 37.86 & 0.49 & 1.69 & 7.13 & 26.7 & 0.051 & 2.21 & 1.15 & 0.397 & 0.10 & 4.64 & & & 134 \\
\hline DP17503 & 5 & 4 & 0.86 & 38.66 & 0.51 & 1.59 & 6.90 & 27.3 & 0.051 & 2.05 & 1.14 & 0.377 & 0.09 & 4.17 & 0 . & & 114 \\
\hline DP17509 & 5 & 5 & 0.46 & 39 & 0.49 & 1.70 & 7.11 & 27.4 & 0.050 & & & & & & & & 23 \\
\hline DP17511 & 5 & 5 & 0.86 & 40.16 & 0.43 & 1. & 6. & 27 & & & & & & & & & 153 \\
\hline DP17513 & 5 & 5 & 1.28 & 40.58 & 0.33 & 1.83 & 7.28 & 26.8 & 0.044 & 2.52 & 0.59 & 0.413 & 0.10 & 4.16 & 0. & 6 & 155 \\
\hline DP17515 & 5 & 6 & 0.06 & 40.86 & 0.44 & 1.88 & $7 \cdot 32$ & 26.5 & 0.045 & 2.62 & 0.58 & 0.408 & 0.10 & 4. & 0.087 & 31 & 126 \\
\hline DP17517 & 5 & 6 & 0.46 & 41.26 & 0.41 & 1.65 & 6.95 & 27.1 & 0.051 & 2.35 & 0.72 & 0.3 & 0.09 & 4. & & & 153 \\
\hline DP17519 & 5 & 6 & 0.86 & 41.66 & 0.49 & 1.77 & 7. & 26.5 & 0.051 & 2. & & & 0. & & & & 131 \\
\hline DP17521 & 5 & 6 & 1.26 & 42.06 & 0.46 & 1.78 & & 26.7 & 0.051 & 2.46 & & & 9 & & & & 138 \\
\hline DP17523 & 5 & 7 & 0.22 & 42.52 & 0.62 & 1.27 & 6.11 & 27.6 & 0.045 & 2.57 & 0.69 & 7 & 0. & 3.43 & 0 & 3 & 119 \\
\hline DP17525 & 6 & 1 & 0.06 & 42.86 & 0.45 & 1.49 & 6.67 & 27.8 & 0.048 & 2.40 & 0.67 & 0.342 & 0.12 & 3.90 & 0 . & 46 & 152 \\
\hline DP17527 & 6 & 1 & 0.67 & 43.47 & 0.45 & 1.62 & 6.10 & 27.2 & 0.045 & 2.21 & 0.63 & 0.3 & 0.14 & 4. & 0 . & 64 & 168 \\
\hline & 6 & 1 & 1.06 & 43.86 & 0.44 & 1.77 & 6.91 & 26.9 & 0.048 & 2.46 & 0.63 & 0.374 & 0.37 & 5 & & 71 & 149 \\
\hline DP17531 & 6 & 1 & 1.46 & 44.26 & 0.57 & & & 27.6 & 0.055 & 2.55 & & & & & & & 25 \\
\hline DP17533 & 6 & 2 & 0.26 & 44.56 & 0.45 & 1.62 & 6. & 27 & 2 & & & & 2 & 7 & 1 & 9 & 153 \\
\hline DP17535 & 6 & 2 & 0.66 & 44.96 & 0.58 & 1.47 & 6.51 & 27.4 & 0.045 & 2.54 & 0.64 & 0. & 0.12 & 3.58 & 0. & 0.061 & 109 \\
\hline DP17537 & 6 & 2 & 1.06 & 45.36 & 0.44 & 1.45 & 6.32 & 27.5 & 0.047 & 2.29 & 0.79 & 0.336 & 0.10 & 3.72 & 0.094 & 0.045 & 150 \\
\hline & 6 & 2 & 1.46 & 45.76 & 0.51 & 1.51 & 6.74 & 27.7 & 0.051 & 2.07 & 1.1 & 0.363 & 0.10 & 4.19 & 0 . & 19 & 156 \\
\hline & 6 & 3 & 0.26 & 46.06 & 0.52 & 1.63 & 6.83 & 27.0 & 0.051 & 2.51 & 0 . & & & & & & 128 \\
\hline DP17543 & 6 & 3 & 0.63 & 46.43 & 0.46 & 1.77 & 7.15 & 26.7 & 0 & 6 & & & 8 & 4 & & & 133 \\
\hline DP17549 & 6 & 4 & 0.24 & 47.54 & 0.54 & 1.63 & 6.99 & 27.1 & 0.047 & 2.17 & 1 . & 86 & 0.09 & 4.07 & 0.083 & 0.050 & 119 \\
\hline DP17551 & 6 & 4 & 0.66 & 47.96 & 0.52 & 1.45 & 6.49 & 27.1 & 0.048 & 2.22 & 0.79 & 0.328 & 0.09 & 3.56 & 0.086 & 0.072 & 136 \\
\hline 53 & 6 & 4 & 1.06 & 48.36 & 0.55 & 1.99 & 5.78 & 26.7 & 0.044 & 2.07 & 0.5 & 0. & 0.20 & 6. & 7 & & 159 \\
\hline & ह & 4 & & 76 & 0.58 & 1.49 & 6.74 & 26.9 & 0.051 & 2.25 & 0 . & & 0 . & & & & 119 \\
\hline 557 & 6 & 5 & 0.26 & 45 & 0.52 & 1.57 & & 27 & 64 & 2.16 & & & 0.09 & & & & 51 \\
\hline DP17559 & 6 & 5 & 0.66 & 49.46 & 0.57 & 1. & & 26 & 1 & 2 & 4 & & 0.12 & 8 & & & 139 \\
\hline DP17563 & 6 & 5 & 1.46 & 50.26 & 0.33 & 1.73 & 6.68 & 27.1 & 0.046 & 2.30 & 0.59 & 0 . & 0.11 & 4.55 & 0.092 & 0.082 & 178 \\
\hline DP17565 & 6 & 6 & 0.34 & 50.64 & 0.68 & 1.94 & 7.11 & 26.0 & 0.058 & 2.15 & 0.9 & 0 . & 4.38 & 3.56 & 0. & 0.056 & 123 \\
\hline DP17567 & 6 & 6 & 0.86 & 51.16 & 0.52 & 1.3 & 6.43 & 27.3 & 0.048 & 2.28 & 0.8 & 0. & 0.08 & 3.32 & 5 & 54 & 139 \\
\hline DP1 & 6 & 7 & 0.03 & 51.83 & 0.50 & 1.6 & 6.74 & 26.7 & 0.047 & 2.23 & 0.8 & 78 & 0.11 & 4.25 & 8 & & 134 \\
\hline DP & 6 & 7 & 0.43 & 52.23 & 0.52 & 1 . & 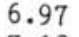 & 26 & 0.0 & 2. & 1 & & 0. & & & & 133 \\
\hline & 7 & 1 & 0.46 & .66 & 0.44 & 1 . & & 26.6 & 0.050 & 2.41 & 0.81 & & 0.10 & 4 & & 0.065 & 145 \\
\hline DP17577 & 7 & 1 & 1.26 & 53.46 & 0.42 & 1.62 & 6.76 & 27.3 & 0.052 & 2.19 & 0.89 & 0.378 & 0.11 & 4.26 & 0.094 & 0.057 & 164 \\
\hline DP17581 & 7 & 2 & 0.46 & .16 & 0.37 & 1.83 & 7.16 & 26.5 & 0.050 & 2.46 & 0.6 & 0.393 & 0.10 & 4.41 & 0.0 & 0.075 & 147 \\
\hline 7583 & 7 & 2 & 0.86 & 54.56 & 0.63 & 1.4 & & 27.2 & 0.047 & 1.80 & 1. & & 0.11 & 4 & & & 130 \\
\hline & 7 & 2 & 1.31 & 55.01 & 0.45 & 1.66 & 6.75 & 27.3 & 0.051 & 2.23 & 0.80 & 0.377 & 0.11 & 4.56 & 0.088 & 0.057 & 164 \\
\hline DP17589 & 7 & 3 & 0.46 & 55.66 & 0.50 & 1.56 & 6.82 & 27.4 & 0.051 & 2.13 & 0.98 & 0.378 & 0.11 & 3.82 & 0.091 & 0.053 & 139 \\
\hline DP17591 & 7 & 3 & 0.86 & & 0.46 & & & 27.4 & 0.050 & & 1.12 & & 0.11 & 4.33 & 0.089 & 0.047 & 174 \\
\hline DP17593 & 7 & 3 & 1.26 & 56.46 & 0.56 & 1.48 & & 27.2 & 0.049 & 1.88 & 1.3 & & 0.11 & & 0.090 & 0.051 & 151 \\
\hline & 7 & 4 & & 56.76 & 0.52 & 1.57 & & 27.3 & 0.052 & 2.03 & 0.9 & 0.343 & 0.13 & 4.77 & 0.086 & 0.058 & 160 \\
\hline DP17599 & 7 & 4 & 0.86 & 57.56 & 0.47 & 1.71 & & & 051 & 2.32 & 0.78 & 0.388 & 0.12 & 4.40 & 0.089 & 0.054 & 137 \\
\hline
\end{tabular}


Table 6. (Continued).

\begin{tabular}{|c|c|c|c|c|c|c|c|c|c|c|c|c|c|c|c|c|c|}
\hline Lab ID & రั & $\begin{array}{l}\text { ¿ } \\
\text { İ } \\
\text { ஸू }\end{array}$ & 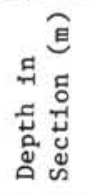 & 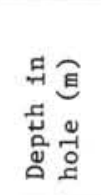 & $\mathrm{Na}$ & $\mathrm{Mg}$ & Al & Si & P & K & $\mathrm{Ca}$ & Ti & Mn & $\mathrm{Fe}$ & $\mathrm{Ba}$ & $\mathrm{S}$ & $\mathrm{H}_{2} \mathrm{O}$ \\
\hline P17601 & 7 & 4 & .26 & .90 & 0.70 & 1.30 & 6.53 & 27.7 & .054 & .07 & .22 & 0.328 & 0.11 & 3.63 & 0.090 & 53 & 124 \\
\hline P17602 & 7 & 4 & .46 & & & 56 & & 6.6 & & 25 & 85 & 363 & 11 & & & & 128 \\
\hline P17603 & 7 & 5 & 0.06 & & 0.62 & 1.48 & & 27.2 & & .16 & .91 & & & & & .053 & 122 \\
\hline DP17605 & 7 & 5 & 0.46 & & 0.59 & & 6.45 & 27.2 & .052 & .96 & .29 & 0.340 & 0.10 & & & & 138 \\
\hline DP17609 & 7 & 5 & 1.26 & & 0.62 & & & & & & & & & & & & 133 \\
\hline P1 & 7 & 6 & 0.06 & & 0 & 2 & & & & & & & & & & & 41 \\
\hline & 7 & 6 & 0.45 & & 0.83 & 1.05 & 5.89 & 28.0 & 7 & 1.78 & & & & & 80 & & 104 \\
\hline 17615 & 7 & 6 & 0.86 & & 0.60 & 1.53 & 6.32 & 27.5 & .048 & 2.02 & .99 & .344 & .11 & & & & 137 \\
\hline 617 & 7 & 6 & 1.26 & .96 & 0.53 & 1.73 & 7.08 & 27.0 & 52 & 2.44 & & & & & & & 127 \\
\hline 619 & 7 & 7 & 0.11 & & 0.66 & 1.47 & 6.51 & & & & & & & & & & 114 \\
\hline DP & 7 & 7 & 0.46 & & 0 & 1.7 & 6. & & & & & & & & & & 46 \\
\hline 25 & 8 & 1 & 0.92 & 2 & 0 & & & & & & & & & & & & 65 \\
\hline & 8 & 1 & 1.26 & 06 & 0.47 & 1.97 & 7.30 & 26.7 & 0.050 & 2.53 & 0.69 & .393 & 1.40 & & & & 128 \\
\hline & 8 & 2 & 0.06 & & 0.52 & 1.54 & 6.82 & 27.1 & 0.045 & 2.55 & 75 & & 0.09 & & & & 122 \\
\hline & 8 & 2 & 0.46 & & 0.57 & 1.59 & 6.24 & & & & & & & & & & 144 \\
\hline DP & 8 & 2 & 0.86 & & 0.39 & 1.73 & 7. & & & & & & & & & & 147 \\
\hline & 8 & 2 & 1.26 & & 0.53 & & & & & & & & & & & & 132 \\
\hline 637 & 8 & 3 & 0.06 & 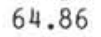 & 0.81 & 0.89 & 6.2 & 1.0 & 4 & .76 & 1. & & & & & & 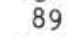 \\
\hline & 8 & 3 & 0.46 & & 0.47 & 1.90 & 7.28 & 26.5 & 9 & .53 & 0. & & & & & & 129 \\
\hline & 8 & 3 & 0.86 & & 0.47 & 1.50 & 6.64 & 27.6 & & 2.19 & & & & & & & 157 \\
\hline 2 & 8 & 3 & 1.26 & & 0.52 & & 7. & & & & & & & & & & 131 \\
\hline & 8 & 4 & 0.06 & & 0. & & & & & & & & & & & & 48 \\
\hline 1 & 8 & 4 & 0.48 & & 0.72 & . & 5. & & & & & & & & & & 107 \\
\hline 49 & 8 & 4 & 0.86 & & 0.47 & 1.5 & 6.54 & 7.2 & 050 & & & & 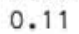 & & & & 48 \\
\hline & 8 & 4 & 1.26 & & 0.47 & 1.7 & 6.96 & & & & & & & & & & 137 \\
\hline & 8 & 5 & 0.06 & & 0.46 & 1.60 & 6.82 & 27.1 & 1 & & & & & & & & \\
\hline & 8 & 5 & 0.46 & & 0.53 & & & & & & & & & & & & 23 \\
\hline & 8 & 5 & 0 & & & & & & & & & & & & & & 74 \\
\hline 659 & 8 & 5 & 1.23 & & 0.54 & 1.2 & 6. & .0 & 4 & 2. & & & & & & & 135 \\
\hline 661 & 8 & 6 & 0.06 & & 0.58 & 1.45 & 6.46 & 5.7 & .051 & a & & & & & & & 139 \\
\hline & 8 & 6 & 0.46 & & 0.59 & 1.69 & 7.08 & & & & & & & & & & 107 \\
\hline & 8 & 6 & 0.91 & & 0.63 & 1.47 & 6.65 & & 3 & 7 & & & & & & & 29 \\
\hline & 8 & 6 & 1.26 & & & & & & & & & & & & & & 112 \\
\hline & 8 & 7 & .05 & & c & & & & & & & & & 3 & 7 & & 111 \\
\hline & 8 & 7 & 0.36 & & 0.82 & 1.2 & 6. & .1 & 47 & 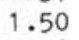 & 1. & & & & & & 104 \\
\hline & 9 & 1 & 0.31 & & 0.53 & 1.7 & & & & & & & & & & & 23 \\
\hline & 9 & 1 & 1.26 & & 0.56 & 1.53 & 6. & 26.6 & 50 & & & & & & & & 31 \\
\hline & 9 & 1 & & & & 1.54 & & & & & & & & & & & 123 \\
\hline & 9 & 2 & 6 & & & & & & & & & & & 8 & & & 123 \\
\hline & 9 & 2 & 0.67 & & c & & 3 & 1 & 0 & 3 & & & 2 & 4.82 & 8 & & 139 \\
\hline & 9 & 2 & 1.06 & & 0.49 & 1.62 & 6. & 26.9 & 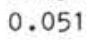 & 31 & 0. & & 7 & 4.33 & & & 130 \\
\hline & 9 & 2 & 1.46 & & 0.6 & & & & & & & & & & & & 1 \\
\hline & 9 & 3 & 0.26 & & 0.82 & 1.21 & 6. & 27.5 & 77 & & & & & & & & 12 \\
\hline & 9 & 3 & & & & & & & & & & & & & & & 128 \\
\hline & 0 & 2 & .46 & & & & & & & & & & & 0 & & & 151 \\
\hline & 9 & 4 & 0.66 & & 0.60 & 1.5 & 6 & 27.5 & 1 & & & & & & & & 136 \\
\hline & 9 & 4 & 1.0 & & 0.6 & & & 26.9 & 1 & & & & & & & & 117 \\
\hline & 9 & 4 & 1.4 & & 0. & & & & & & & & & & & & \\
\hline & 9 & 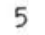 & & & & & & & & & & & & & 6 & 1 & 112 \\
\hline & 9 & 5 & 66 & & & 1 & & & & & & & & 4.80 & 9 & & 133 \\
\hline & 9 & 5 & & & 3 & & & 26.7 & & & & & & & & & 149 \\
\hline & 9 & 6 & 0.46 & & 0.75 & & 6 & & & & & & 0.11 & & & & 99 \\
\hline & 9 & 6 & 0.86 & & 0.60 & 1. & 6. & 26.9 & 0.0 & 2. & & & & & & & 117 \\
\hline & 9 & 6 & & & & & & & & & & & & & & & 124 \\
\hline & 0 & 7 & & & & & & 27.3 & & & & & & & 90 & 0. & 113 \\
\hline & 10 & 9 & & & & & & & & & & & & 4.32 & 0. & 51 & 121 \\
\hline & & 1 & & & & & & & & & & & & & & & 126 \\
\hline & 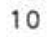 & 1 & $\pi$ & & 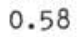 & & & 26.8 & .048 & & & & & 4.64 & 0.0 & 0.051 & 125 \\
\hline & 10 & 1 & 0.9 & & 0.5 & & & 26.9 & 049 & & & & 0.15 & & & & 143 \\
\hline 17. & 10 & 1 & 1.3 & & 0. & & & 27.3 & & & & & & & & & \\
\hline 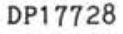 & 10 & 1 & & & & & & 27.0 & & & & & & & & & 140 \\
\hline & 10 & 2 & 0 & & & & & & & & & & & & & 033 & 136 \\
\hline & 10 & 2 & & & & & & & & & & & 0. & & & & 111 \\
\hline & 10 & 2 & & & & & & 26.3 & 0.067 & . & 2.13 & 0.427 & 0.55 & 4.55 & 0.086 & 0.045 & 90 \\
\hline
\end{tabular}


Table 6. (Continued).

\begin{tabular}{|c|c|c|c|c|c|c|c|c|c|c|c|c|c|c|c|c|c|}
\hline Lab ID & 㫕 & 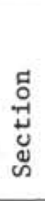 & 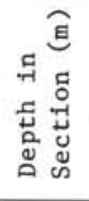 & 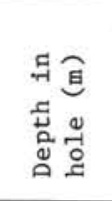 & $\mathrm{Na}$ & $\mathrm{Mg}$ & Al & Si & $\mathrm{P}$ & K & $\mathrm{Ca}$ & Ti & Mn & $\mathrm{Fe}$ & $\mathrm{Ba}$ & $\mathrm{s}$ & $\mathrm{H}_{2} \mathrm{O}$ \\
\hline 733 & 10 & 2 & 86 & 83.16 & .58 & 1.48 & .74 & 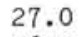 & & .96 & .09 & 376 & 16 & 43 & .082 & .044 & . \\
\hline 735 & 10 & 2 & 26 & & & & & & & & & & & & & & \\
\hline 17736 & 10 & 2 & 1.0 & & & & & & & & & & & & .077 & & 113 \\
\hline P17737 & 10 & 3 & 0.06 & & 0.00 & 1. & & & & & & - 501 & 1 & .85 & .080 & & 121 \\
\hline 7739 & 10 & 3 & .46 & & .52 & 1.56 & & 27.0 & & & & & .18 & & & & 133 \\
\hline DP17741 & 10 & 3 & 0.86 & & 0.48 & 1.65 & 7.23 & 26.5 & 52 & .34 & 0.74 & & & & & & 129 \\
\hline DP17742 & 10 & 3 & 1.06 & & 0.58 & 1.5 & & & & & & & & & & & 129 \\
\hline 17743 & 10 & 3 & 1.26 & & 055 & & & & & & & & & & & & 131 \\
\hline & 10 & 4 & 0.06 & 8 & 5 & & & 26. & 3 & & & & 0.37 & & & & 127 \\
\hline & 10 & 4 & 0.46 & & 0.52 & 1. & & 27.1 & & 27 & 0 & & 21 & & & & 132 \\
\hline & 10 & 4 & 0.86 & & 0.72 & 1.37 & & & & & & & & & & & 117 \\
\hline & 10 & 4 & 1.26 & & 0.67 & & & & & & & & & & & & 25 \\
\hline 753 & 10 & 5 & 0.06 & & 0. & 1.41 & & & & & & & & & 4 & & 131 \\
\hline & 10 & 5 & 0.46 & 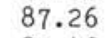 & 2 & & & & & & & & & & & & 137 \\
\hline & 10 & 5 & .66 & & .30 & 1.52 & 0.00 & 26.6 & כ2 & 7 & 0.8 & & .33 & 4.39 & & & 139 \\
\hline & 10 & 5 & 0.86 & & .56 & 1.49 & 6.81 & 26.3 & 49 & 7 & & & & & & & 123 \\
\hline & 10 & 5 & 1.06 & & .60 & & & 26.8 & & & & & & & & & 129 \\
\hline 9 & 10 & 5 & 1.26 & & 0.55 & 1. & & & & & & & & & & & 131 \\
\hline 1 & 10 & 6 & 0.06 & & & & & & & & & & 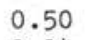 & & & & 132 \\
\hline & 10 & 6 & .46 & & .33 & 1. & 7.02 & 26. & & & 1. & & & & & & \\
\hline & 10 & 6 & 0.86 & & .51 & 1.54 & 6.95 & 26.5 & & & & & & & & & 138 \\
\hline & 11 & 1 & 0.26 & & .48 & 1.44 & 6.75 & 27.1 & & & & & & & & & 131 \\
\hline & 11 & 1 & 0.66 & & & & & & & & & & & & & & 12 \\
\hline 1 & 11 & 1 & .06 & & 7 & & & & & & 2 & & 0 & & & & \\
\hline & 11 & 1 & .43 & & 5 & 1. & 6. & & & & & & & & & & 143 \\
\hline & 11 & 2 & .26 & & .48 & & 6.75 & 26.5 & & & & & & & & & 139 \\
\hline & 1 & 2 & .66 & & .69 & 1. & & & & 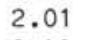 & & & & & & & - \\
\hline & 1 & 2 & 106 & 36 & 2 & 1. & & & & & & & & & & & 13 \\
\hline & 11 & 2 & 46 & & & & & & & & 0. & & & & & & \\
\hline & 1 & 3 & 0.06 & & 8 & & 8 & 27.2 & & 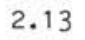 & 0. & & & & & & \\
\hline & 11 & 3 & 0.26 & & .49 & 1.4 & .86 & 26.9 & 48 & & & & & & & & 136 \\
\hline & 11 & 3 & 0.66 & & 45 & & 6. & & & & & & & & & & \\
\hline & 11 & 3 & 1.06 & & 54 & & 6.60 & 2 & 0 & 5 & & & & & & & 129 \\
\hline & 11 & 3 & 1.26 & & 6 & & 7 & & & & & & & & & & 150 \\
\hline & 1 & 3 & 46 & & & & & & & & 0.83 & & 0. & & & & 13 \\
\hline & 11 & 4 & .26 & & .48 & & & 26.8 & 0.0 & 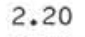 & 0.66 & & .19 & & & & \\
\hline & 11 & 4 & 0.66 & & 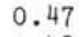 & 1 & 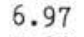 & 26.4 & & 0 & 0.66 & & & & & & 129 \\
\hline & 11 & 4 & 1.06 & & & & & & & & & & & & & & \\
\hline & 11 & 4 & .46 & & & & & & & & & & & & & & 12 \\
\hline & & 5 & 06 & & & & & & & & & & 0 & 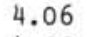 & & & 11 \\
\hline & & 5 & .26 & & & & & & & 2.45 & 0. & & 0. & 3 & & & 13 \\
\hline & 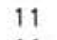 & 5 & .66 & & 0 & & 7. & 26.8 & 0.05 & 2.41 & & & & & & & \\
\hline & 11 & 6 & 0.26 & & 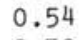 & & 6. & 26.6 & 0. & & 0.85 & 3 & & & & & 12 \\
\hline & 11 & 6 & 0.66 & & . & & & & & & & & & & & & 10 \\
\hline & 11 & 6 & & & & & & & & & & & & & & & \\
\hline & & 6 & & & & & & & & & & & 0. & 2 & & & 13 \\
\hline & 1 & 6 & 16 & & & & 6. & & & 2. & & & 0. & & & & 13 \\
\hline & 1 & 7 & .2 & & 3 & & 5.8 & 28 & & & & & & & & & \\
\hline & 12 & 1 & .96 & & & & & & & & & & & & & & 10 \\
\hline & 12 & 1 & & & & & & & & & & & & & & & \\
\hline & & 2 & & & & & & & & 2. & 1 & & 0. & & & & 7 \\
\hline & 1 & 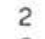 & 24 & & & & & 2 & & 2. & & & & & & & 14 \\
\hline & 12 & 2 & .67 & & & & 6. & 26 & & & 1. & & 0.14 & & & & 11 \\
\hline 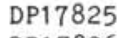 & 12 & 2 & 1.06 & .64 & 0.52 & & 6. & & & & & & & & & & \\
\hline & 12 & 2 & & & & & & & & & & & & & & & 12 \\
\hline & 1 & 2 & & & & & & & & & & & & & & & \\
\hline & 1 & 3 & & & & & & & & & & & 0.15 & 4.23 & & & 13 \\
\hline & 12 & 3 & .66 & & 0. & & 6. & & & & & & & & & & \\
\hline & 12 & 3 & זו.10 & & . & & & & & & & & & & & & \\
\hline 7834 & 12 & 4 & & 3.64 & 0. & & & & & & & & & & & & \\
\hline & 12 & 4 & & & 0. & & & 26.4 & & & & & & & & & 12 \\
\hline & 12 & 4 & & & & & & & & & & & & & & & 13 \\
\hline & 12 & 4 & 1.00 & & 0. & & 7.18 & 26.5 & 0.0 & 2. & 0.72 & & 0.33 & 4.54 & 0.077 & & 12 \\
\hline 17841 & 12 & 4 & .46 & 05.04 & 0.62 & & 6.29 & 27.4 & 0.043 & 2.79 & 0.52 & 0.262 & 0.26 & 2.91 & 0.088 & 0.027 & \\
\hline
\end{tabular}


Table 6. (Continued).

\begin{tabular}{|c|c|c|c|c|c|c|c|c|c|c|c|c|c|c|c|c|c|}
\hline Lab ID & 㫕 & 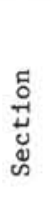 & 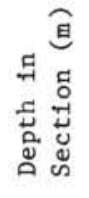 & 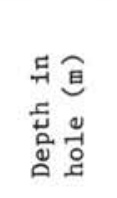 & $\mathrm{Na}$ & $\mathrm{Mg}$ & A1 & $\mathrm{S} 1$ & $\mathrm{P}$ & K & $\mathrm{Ca}$ & $\mathrm{T} 1$ & Mn & $\mathrm{Fe}$ & $\mathrm{Ba}$ & $\mathrm{S}$ & $\mathrm{H}_{2} \mathrm{O}$ \\
\hline DP17843 & 12 & 5 & 26 & 5.34 & 0.51 & 1.58 & 7.26 & 26.7 & 0.058 & 2.45 & 0.57 & 0.366 & .34 & 4.30 & 0.076 & 48 & 119 \\
\hline DP17845 & 12 & 5 & .66 & 105.74 & 0.54 & 1.63 & 7.17 & & .057 & 2.42 & & & & & & & \\
\hline DP17847 & 12 & 5 & 1.06 & 106.14 & 0.46 & 1.60 & 7.12 & 26.8 & 0.063 & 2.39 & 0.61 & 0.366 & 40 & 4.24 & 0.081 & 43 & 131 \\
\hline DP17849 & 12 & 5 & 1.46 & 106.54 & 0.50 & 1.75 & 7.3 & 26.4 & 0.061 & 2.47 & 0.54 & 0.382 & .58 & 4.45 & 0.088 & & 126 \\
\hline DP17851 & 12 & 6 & 0.86 & 107.44 & 0.58 & 1.25 & 6.60 & $27 \cdot 3$ & 0.046 & 2.78 & 0.54 & 0.288 & 0.28 & 3.34 & 0.085 & 0.034 & 100 \\
\hline DP17853 & 12 & 6 & 1.23 & 107.81 & 0.48 & 1.67 & 7.37 & 26.3 & 0.055 & 2.40 & 0.55 & 0.381 & 0.95 & 4.48 & 0.093 & 0.044 & 123 \\
\hline DP17855 & 13 & 1 & 0.26 & 109.56 & 0.42 & 1.37 & 6.89 & 26.7 & 0.049 & 2.46 & 0.47 & 0.338 & 0.18 & 3.97 & 0.082 & 0.038 & 125 \\
\hline DP17856 & 13 & 1 & 0.46 & 109.76 & 0.39 & 1.53 & 7.29 & 26.8 & 0.051 & 2.43 & 0.45 & 0.380 & 0.51 & 4.43 & 0.085 & 35 & 138 \\
\hline DP17857 & 13 & 1 & 0.66 & 109.96 & 0.40 & 1.45 & 7.11 & 26.8 & 0.050 & 2.39 & 47 & 365 & & 4.32 & 0.085 & 3 & 135 \\
\hline 58 & 13 & 1 & 0.86 & 110.16 & 0.41 & 1.43 & 6.93 & 26.9 & 0.049 & 2.44 & 0.47 & 2 & & 4.17 & & & 136 \\
\hline & 13 & 1 & 1.06 & 110.36 & 0.36 & 1.44 & 6.98 & 26.9 & 0.049 & 2.37 & 0.43 & 0.363 & 0.21 & 4.30 & 0.083 & 0.030 & 142 \\
\hline 60 & 13 & 1 & 1.26 & 110.56 & 0.43 & 1.46 & 6.98 & 26.9 & 0.051 & 2.45 & 0.45 & & 0.27 & & & 0.031 & 125 \\
\hline 861 & 13 & 1 & 1.46 & 110.76 & 0.45 & 1.35 & 6.76 & 27.2 & 0.048 & 2.44 & 0.47 & 0.330 & 0.39 & 3.90 & 0. & 0. & 131 \\
\hline 362 & 13 & 2 & 0.06 & 110.86 & 0.38 & 1.48 & 7.02 & 27.0 & 0.051 & 2.41 & 4 & & & 4.28 & 0.089 & & 146 \\
\hline 63 & 13 & 2 & 0.26 & 111.06 & 0.41 & 1. & 7.02 & 27.2 & 0.051 & 2.38 & 0.49 & 4 & 0.32 & 4.25 & 0.086 & 39 & 145 \\
\hline 64 & 13 & 2 & 0.46 & 111.26 & 0.42 & 1.50 & 7.07 & 26.8 & 0.050 & 2.42 & 0.47 & 5 & 0.27 & 4.37 & & & 138 \\
\hline & 13 & 2 & 0.66 & 111.46 & 0.40 & 1.49 & 7.12 & 26.9 & 0.051 & 2.37 & 0.52 & & 0.23 & 4.46 & 0. & & 140 \\
\hline 66 & 13 & 2 & 0.86 & 111.66 & 0.42 & 1.56 & 7.31 & 26.9 & 0.051 & 2.41 & 0.56 & 0.382 & 0.23 & 4.58 & 0.077 & & 134 \\
\hline 67 & 13 & 2 & 1.06 & 111.86 & 0.44 & 1.55 & 7.16 & 26.1 & 0.0 & 2.38 & 0.48 & 0.369 & 0. & 4.47 & 35 & & 131 \\
\hline 8 & 13 & 2 & 1.26 & 112.06 & 0. & 1.52 & 7.17 & 26.9 & 0.0 & 2. & 6 & 2 & 3 & 4.40 & 39 & 7 & 138 \\
\hline 69 & 13 & 2 & 1.46 & 112.26 & 0.38 & 1.52 & 7.17 & 26.7 & 0.048 & 2.36 & 0.47 & 0.369 & 3 & 4.42 & 0. & & 137 \\
\hline 70 & 13 & 3 & 0.06 & 112.36 & 0.40 & 1.58 & 7.36 & 26.9 & 0.049 & 2.43 & 0.49 & & 4 & 4.57 & & & 129 \\
\hline & 13 & 3 & 0.26 & 112.56 & 0.38 & 1.50 & 7.07 & 26.4 & 0 . & 2.35 & 0.52 & 2 & 0.32 & 4.54 & 9 & 7 & 140 \\
\hline 72 & 13 & 3 & 0.46 & 112.76 & 0.34 & 1.52 & 7.19 & 26.8 & 0.0 & 2.37 & 0.49 & 0.378 & 0.26 & 4.55 & 3 & & 141 \\
\hline 873 & 13 & 3 & 0.66 & 112.96 & 0.45 & 1.81 & 7. & 26.2 & 0.049 & 2.34 & 0.49 & 2 & 2 & 4. & 7 & 3 & 120 \\
\hline 4 & 13 & 3 & 0.86 & 113.16 & 0.42 & 1 & 7.21 & 26.3 & 0.049 & 2.35 & 0.52 & 5 & 0.19 & 4.53 & 0.0 & & 135 \\
\hline 875 & 13 & 3 & 1.06 & 113.36 & 0.37 & 1.60 & 7.33 & 26.5 & 0.051 & 2.43 & 0.47 & 35 & 0.30 & 4.57 & 0.082 & 0. & 136 \\
\hline 376 & 13 & 3 & 1.26 & 113.56 & 0.41 & 1.50 & 7.09 & 26.8 & 0.0 & 2.30 & 0.58 & 6 & 0.30 & 4. & 8 & & 146 \\
\hline 77 & 13 & 3 & 1.46 & 113.76 & 0.38 & 1.55 & 7.23 & 27.0 & 0.049 & 2.42 & 0.52 & 0.385 & 0.22 & 4.60 & 6 & & 138 \\
\hline DP17878 & 13 & 4 & 0.06 & 113.86 & 0.38 & 1.51 & 6.92 & 26.2 & 0.048 & 2. & 0.60 & 4 & 7 & 4.54 & 0. & 0. & 143 \\
\hline DI & 13 & 4 & 0.26 & 114.06 & 0 . & 1 & 7. & 7 & 0. & 2.32 & 0.63 & 0. & 0.20 & 4.63 & 0. & & 130 \\
\hline 380 & 13 & 4 & 0.46 & 114.26 & 0. & & 6. & 26.9 & 0.047 & 2.34 & 0.51 & 0.363 & 0.25 & 4.40 & 0.082 & & 141 \\
\hline 881 & 13 & 4 & 0.66 & 114.46 & 0.39 & 1.34 & 6.69 & 27.0 & 0.045 & 2.43 & 0.60 & 0.3 & 0.16 & 3.99 & 0.085 & 0. & 129 \\
\hline 82 & 13 & 4 & 0.86 & 114.66 & 0.40 & 1.31 & 6.72 & 27.2 & 0.046 & 2.50 & 0.55 & 0. & 0.23 & & 3 & & 116 \\
\hline 83 & 13 & 4 & 1.06 & 114.86 & 0.46 & 1.54 & 7.22 & 26.6 & 0.049 & 2.40 & 0.50 & 0.3 & 0.34 & 3 & 34 & 0 & 131 \\
\hline DP17884 & 13 & 4 & 1.26 & 115.06 & 040 & 1.54 & 7.25 & 26.5 & 0.0 & 2 & 3 & & 0.23 & 4.63 & 0.076 & 0. & 136 \\
\hline & 13 & 4 & 1.46 & .26 & 0. & 8 & 7. & 26.5 & 0 . & 2. & 0 & 0 & 0.18 & 4.76 & 0.082 & 0. & 124 \\
\hline 7886 & 13 & 5 & 0.06 & 15.36 & 0.44 & 1.66 & 7.14 & 26.6 & 0.048 & 2.22 & 0.77 & 0 & 0.16 & 4.97 & 0.078 & 0. & 139 \\
\hline 7887 & 13 & 5 & 0.26 & 115.56 & 0.47 & 1.53 & 7.09 & 26.9 & 0.049 & 2.42 & 0.58 & 0.360 & 0.16 & $4 \cdot 30$ & 0.084 & 0.047 & 123 \\
\hline 388 & 13 & 5 & 0.46 & 115.76 & 0.50 & 1.62 & 7.16 & 26.6 & 0.048 & 2.32 & 0.66 & 0.387 & 0.18 & & 0.077 & & 31 \\
\hline & 13 & 5 & 0.66 & 115.96 & 0.45 & 1.58 & $7 \cdot 32$ & 26.7 & 0.051 & 2.36 & 0.58 & 2 & 8 & 4. & 0. & & 126 \\
\hline DP17890 & 13 & 5 & 0.86 & 116.16 & 0.42 & 1.56 & 7.1 & 26 & 9 & 2. & 0.63 & 0 & 0 . & 4.48 & 0.079 & 0. & 128 \\
\hline 891 & 13 & 5 & 1.06 & .36 & 0.47 & 1.77 & 7.43 & 26.3 & 50 & 2.32 & 0.63 & 0.406 & 0.20 & 4.95 & 0.083 & 0.0 & 131 \\
\hline 92 & 13 & 5 & 1.26 & 116.56 & 0.41 & 1.56 & 7.25 & 26.6 & 0.052 & 2.33 & 0.52 & 0.381 & 0.18 & 4.57 & 0.079 & & 142 \\
\hline 893 & 13 & 5 & 1.46 & 116.76 & 0.45 & 1.61 & 7.21 & 26.4 & 0.054 & 2.36 & 0.55 & 0.381 & 0.20 & 4.64 & 0.082 & 0.051 & 133 \\
\hline DP17894 & 13 & 6 & 0.06 & 116.86 & 0.36 & 1.55 & 7.20 & 26.7 & 0.053 & 2.30 & 0.59 & & 0.21 & & 8 & & \\
\hline DP17895 & 13 & 6 & 0.26 & 117.06 & 0.47 & 2.08 & 7.42 & 26.1 & 0.052 & 2.13 & .56 & & 9 & 4.57 & 0.078 & 0.062 & 138 \\
\hline & 13 & 6 & 0.46 & 117.26 & 0.38 & 1.54 & 7.0 & 26 & 0. & 2. & 0. & & 0.22 & 4.73 & 0.078 & 0.029 & 145 \\
\hline & 13 & 6 & 0.66 & 117.46 & 0.43 & 1.54 & 7.20 & 26.7 & 0.055 & 2.44 & 0.60 & 0. & 0.26 & 4.48 & 0.084 & 0.0 & 129 \\
\hline 898 & 13 & 6 & 0.86 & 117.66 & 0.49 & 1.48 & 7.01 & 26.5 & 0.052 & 2.52 & 0.61 & 0.339 & 0.25 & 4.16 & 0.080 & & 112 \\
\hline DP17899 & 13 & 6 & 1.06 & 117.86 & 0.57 & 1.65 & 7.12 & 26.3 & 0.053 & 2.14 & 1.08 & 0.405 & 0.21 & 5.19 & 0.082 & & 115 \\
\hline DP17900 & 13 & 6 & 1.26 & 118.06 & 0.55 & 1.75 & 7.34 & 25.9 & 0.057 & 2.15 & 1.02 & & 0.23 & & & & \\
\hline & 13 & 6 & 1.46 & .26 & & 1.70 & 7.17 & 25.9 & 0.054 & & 1.17 & & 0.24 & & 0 . & 0. & 116 \\
\hline & 13 & 7 & 0.06 & & 0. & 1.72 & 7.1 & 25.9 & 0.053 & 2.11 & 1.05 & 0. & 0.27 & $5 \cdot 31$ & 0.084 & 0.042 & 123 \\
\hline & 13 & 7 & 0.26 & & 0.1 & 1. & $7 \cdot 3$ & 26.2 & 0.057 & 2.17 & 0.91 & 0.415 & 0.31 & & 0.088 & 0.050 & 125 \\
\hline DP17904 & 14 & 1 & 0.31 & 118.66 & 0.38 & 1.66 & 7.08 & 26.1 & 0.053 & 1.96 & 1.23 & 0.402 & 0.27 & 5.24 & 0.081 & & 142 \\
\hline DP17905 & 14 & 1 & 0.56 & 118.91 & 0.51 & 1.63 & 6.99 & 26.5 & 0.052 & 2.01 & 1.31 & 0.401 & 0.23 & 5.09 & 0.084 & 0.055 & 118 \\
\hline DP17906 & 14 & 1 & 0.86 & 119.21 & 0.45 & & 7.15 & 26.0 & 0.057 & & & & & & & & \\
\hline & 14 & 1 & 1.06 & 119.41 & 0.56 & 1.38 & 6.64 & 26.8 & 0.047 & 2.35 & 1.27 & 24 & 0.28 & 4.57 & 0.076 & 0.043 & 120 \\
\hline & 14 & 1 & 1.46 & 119.81 & 0.35 & 1.82 & 7.41 & 25.7 & 0.062 & 2.27 & 0.51 & & 0.45 & & 0.088 & 0.055 & 139 \\
\hline DP17909 & 14 & 2 & 0.06 & 119.91 & 0.42 & 1.84 & 7.45 & 25.7 & 0.063 & 2.30 & & & 0.48 & & 0.086 & 0.053 & 191 \\
\hline DP17910 & 14 & 2 & 0.26 & 120.11 & 0.40 & 1.86 & 7.34 & 25.6 & 0.059 & 2.23 & 0.57 & & 0.41 & 4.87 & 0.081 & & 134 \\
\hline DP17911 & 14 & 2 & 0.46 & 120.31 & 0.42 & 1.80 & 7.3 & 26.1 & 0.057 & 2.30 & 0.59 & & 0.37 & 4.67 & & & 133 \\
\hline DP17912 & 14 & 2 & 0.66 & 120.51 & 0.39 & 1.66 & 7.04 & 26.2 & 0.054 & 2.06 & 0.80 & 0.377 & 0.32 & 4.62 & 0.077 & 0.043 & 132 \\
\hline
\end{tabular}


Table 6. (Continued).

\begin{tabular}{|c|c|c|c|c|c|c|c|c|c|c|c|c|c|c|c|c|c|}
\hline Lab ID & 号 & $\begin{array}{l}\text { ㅁ } \\
\text { İ } \\
\text { ஸ் }\end{array}$ & 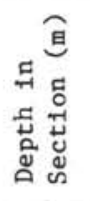 & 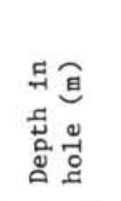 & $\mathrm{Na}$ & $\mathrm{Mg}$ & A1 & S1 & P & K & $\mathrm{Ca}$ & Ti & Mn & $\mathrm{Fe}$ & $\mathrm{Ba}$ & $\mathrm{s}$ & $\mathrm{H}_{2} \mathrm{O}$ \\
\hline DP17913 & 14 & 2 & 0.86 & 120.71 & 0.71 & 1.20 & 6.16 & 27.5 & 0.048 & 1.99 & 1.08 & 0.306 & 0.20 & 3.30 & 0.074 & 0.031 & 92 \\
\hline DP17914 & 14 & 2 & 1.06 & 120.91 & 0.33 & 1.88 & 7.24 & 25.9 & 0.053 & 2.15 & 0.55 & 0.378 & 0.38 & 4.72 & 0.091 & & 152 \\
\hline DP17915 & 14 & 2 & 1.26 & 121.11 & & 1.88 & 7.35 & 26.0 & 0.059 & 2.17 & 0.55 & 0.395 & 0.49 & 4.91 & 0.098 & 0.053 & 158 \\
\hline DP17916 & 14 & 2 & 1.46 & 121.31 & 0.46 & 1.88 & 7.39 & 25.8 & 0.064 & 2.21 & 0.61 & 0.400 & 0.51 & 5.00 & 0.101 & 0.061 & 132 \\
\hline DP17917 & 14 & 3 & 0.06 & 121.41 & 0.45 & 1.88 & 7.37 & 25.7 & 0.060 & 2.22 & 0.60 & 0.402 & 0.43 & 5.01 & 0.100 & 0.058 & 130 \\
\hline DP17918 & 14 & 3 & 0.26 & 121.61 & 0.39 & 1.91 & 7.35 & 26.0 & 0.059 & 2.23 & 0.55 & 0.406 & 0.42 & 5.15 & 0.101 & 0.069 & 138 \\
\hline DP17919 & 14 & 3 & 0.46 & 121.81 & 0.36 & 1.90 & 7.35 & 25.7 & 0.062 & 2.24 & 0.53 & 0.405 & 0.45 & 5.08 & 0.101 & 0.054 & 137 \\
\hline DP17920 & 14 & 3 & 0.66 & 122.01 & 0.44 & 1.87 & 7.40 & 25.7 & 0.064 & 2.24 & 0.62 & 0.408 & 0.41 & 5.11 & 0.096 & & 126 \\
\hline DP17921 & 14 & 3 & 0.86 & 122.21 & 0.46 & 1.86 & 7.41 & 25.8 & 0.065 & 2.28 & 0.59 & 0.405 & 0.42 & 5.07 & .103 & & 118 \\
\hline DP17922 & 14 & 3 & 1.06 & 122.41 & 0.40 & 1.93 & 7.41 & 25.9 & 0.057 & 2.17 & 0.70 & 0.413 & 0.33 & 5.33 & 0.103 & & 141 \\
\hline DP17923 & 14 & 3 & 1.26 & 122.61 & 0.58 & 1.93 & 7.38 & 26.1 & 0.057 & 2.31 & 0.59 & 0.409 & 0.37 & 4.99 & 0.108 & 0.053 & 103 \\
\hline DP17924 & 14 & 3 & 1.46 & 122.81 & 0.57 & 1.85 & 7.26 & 26.0 & 0.053 & 2.20 & 0.68 & 0.386 & 0.35 & 4.93 & 0.113 & & 104 \\
\hline DP17925 & 14 & 4 & 0.06 & 122.91 & 0.53 & 1.86 & $7 \cdot 30$ & 26.0 & 0.056 & 2.34 & 0.44 & 0.393 & 0.54 & 4.76 & 0.108 & 0.050 & 106 \\
\hline DP17926 & 14 & 4 & 0.26 & 123.11 & 0.53 & 1.91 & 7.28 & 25.9 & 0.058 & 2.27 & 0.48 & 0.393 & 0.49 & 4.86 & 0.093 & 0.058 & 109 \\
\hline DP1 & 14 & 4 & 0.46 & 123.31 & 0.55 & 1.98 & 7.26 & 26.1 & 0.055 & 2.13 & 0.57 & 0.378 & 0.46 & 4.73 & 0.078 & 0.055 & 114 \\
\hline DP17928 & 14 & 4 & 0.66 & 123.51 & 0.56 & 1.90 & 7.37 & 25.9 & 0.055 & 2.26 & 0.53 & 0.397 & 0.51 & 4.91 & 0.083 & 0.052 & 112 \\
\hline 929 & 14 & 4 & 0.86 & 123.71 & 0.61 & 1.95 & 7.39 & 26.1 & 0.060 & 2.28 & 0.53 & 0.396 & 0.58 & 4.88 & 0.089 & 0.064 & 105 \\
\hline DP17930 & 14 & 4 & 1.06 & 123.91 & 0.62 & 2.15 & 7.19 & 26.2 & 0.049 & 2.06 & 0.57 & 0.404 & 0.38 & 4.86 & 0.076 & 0. & 116 \\
\hline DP17931 & 14 & 4 & 1.26 & 124.11 & 0.61 & 1.94 & 7.30 & 25.8 & 0.056 & 2.31 & 0.57 & 0.410 & 0.39 & 5.29 & 0.084 & 0.049 & 99 \\
\hline DP17932 & 14 & 4 & 1.46 & 124.31 & 0.59 & 2.01 & 7.15 & 25.4 & 0.044 & 1.66 & 1.30 & 0.479 & 0.19 & 5.87 & 0.071 & 0.045 & 137 \\
\hline DP17933 & 14 & 5 & 0.06 & 124.41 & 0.70 & 1.88 & 7.30 & 25.3 & 0.047 & 1.82 & 1.37 & 0 & 0. & & 0.071 & 0.055 & 122 \\
\hline 7934 & 14 & 5 & 0.26 & 124.61 & 0.64 & 1.86 & 7.32 & 25.8 & 0.056 & 2.31 & 0.57 & 0.408 & 0.48 & 5.09 & 91 & 1 & 93 \\
\hline DP17935 & 14 & 5 & 0.46 & 124.81 & 0.63 & 1.95 & 7.27 & 25.9 & 0.055 & 2.26 & 0.52 & 0.3 & 0.53 & 4 & 0.085 & 19 & 99 \\
\hline DP17936 & 14 & 5 & 0.66 & 125.01 & 0.70 & 1.92 & 7.38 & 26.1 & 0.060 & 2.32 & 0.65 & 0.410 & 0.44 & 5.10 & 0.092 & & 92 \\
\hline DP1 & 14 & 5 & 0.86 & 125.21 & 0.61 & 1.90 & 7.13 & 26.0 & 0.057 & 2.13 & 0.52 & 0.367 & 0.62 & 4.72 & 0.079 & 48 & 107 \\
\hline 7938 & 14 & 5 & 1.06 & 125.41 & 0.61 & 1.86 & 7.13 & 26.1 & 0.060 & 2.18 & 0.48 & 0.385 & 0.58 & 4.75 & 39 & 0.038 & 102 \\
\hline 939 & 14 & 5 & 1.26 & 125.61 & 0.66 & 1.84 & 7.14 & 25.9 & 0.070 & 2.23 & 0.55 & 0.393 & 0.57 & 4.80 & 0.095 & 0.051 & 94 \\
\hline DP17940 & 14 & 5 & 1.46 & 125.81 & 0.70 & 1.87 & 7.25 & 25.9 & 0.070 & 2.20 & 0.66 & 0.401 & 0.51 & 5.07 & 39 & 51 & 94 \\
\hline DP17941 & 14 & 6 & 0.06 & 125.91 & 0.66 & 1.87 & 7.20 & 25.8 & 0.062 & 2.33 & 0.48 & 0.396 & 0.57 & 4.91 & 0. & 6 & 89 \\
\hline DP17942 & 14 & 6 & 0.26 & 126.11 & 0.67 & 1.81 & 7.27 & 25.8 & 0.061 & 2.42 & 0.52 & 0.411 & 0.54 & 4.99 & 0.099 & & 83 \\
\hline DP17943 & 14 & 6 & 0.46 & 126.31 & 0.65 & 1.82 & $7 \cdot 35$ & 25.8 & 0.068 & 2.32 & 0.63 & 0.408 & 0.60 & 5.15 & 0.086 & 0.041 & 89 \\
\hline DP17944 & 14 & 6 & 0.66 & 126.51 & 0.67 & 1.78 & 7.25 & 25.4 & 0.065 & 2.34 & 0.60 & 0.403 & 0.64 & 5.09 & 0.085 & 0.038 & 85 \\
\hline DP17945 & 14 & 6 & 0.86 & 126.71 & 0.74 & 1.88 & 7.35 & 25.6 & 0.061 & 2.38 & 0.53 & 0.406 & 0.67 & 5.18 & 0.084 & .053 & 90 \\
\hline DP17946 & 14 & 6 & 1.06 & 126.91 & 0.59 & 1.87 & 7.34 & 26.0 & 0.068 & 2.29 & 0.52 & 0.390 & 4 & 84 & 71 & 5 & 96 \\
\hline DP17947 & 14 & 6 & 1.26 & 127.11 & 0.65 & 1.93 & 7.34 & 25.7 & 0.062 & 2.30 & 0.43 & .384 & 0.63 & 4.83 & 0.086 & 0. & 97 \\
\hline 7948 & 14 & 6 & 1.38 & 127.23 & 0.67 & 1.80 & 7.40 & 25.7 & 0.064 & 2.45 & 0.51 & 0.411 & 0.64 & 5.05 & 0.086 & 0.042 & 85 \\
\hline DP17949 & 14 & 7 & 0.06 & 127.41 & 0.66 & 1.81 & 7.39 & 25.7 & 0.065 & 2.42 & 0.54 & 0.410 & 0.65 & 5.08 & 0.084 & 0.050 & 87 \\
\hline 7950 & 14 & 7 & 0.26 & 127.61 & 0.66 & 1.79 & 7.39 & 25.7 & 0.068 & 2.46 & 0.47 & 0.403 & 0.70 & 4.97 & 0.089 & 42 & 86 \\
\hline DP17951 & 14 & 7 & 0.44 & 127.79 & 0.69 & 1.81 & 7.45 & 25.8 & 0.067 & 2.43 & 0.53 & 0.408 & 0.73 & 5. & 0 . & 48 & 89 \\
\hline DP17956 & 15 & 1 & 0.44 & 128.44 & 0.61 & 1.84 & 7.34 & 25.2 & 0.064 & 2.25 & 0.51 & 94 & 0.82 & 5.03 & 0.092 & 0.033 & 103 \\
\hline DP1 7957 & 15 & 1 & 0.66 & 128.66 & 0.51 & 2. & 7.41 & 25 & 0.063 & 2. & 0.43 & 0.365 & 0.67 & 4.46 & 0.086 & 0.043 & 122 \\
\hline 958 & 15 & 1 & 0.86 & 128.86 & 0.63 & 1.85 & 7.36 & 25.2 & 0.064 & 2.17 & 0.52 & 0.398 & 0.80 & 4.95 & 0.090 & 0.042 & 107 \\
\hline 7959 & 15 & 1 & 1.08 & 129.08 & 0.64 & 1.86 & 7.44 & 25.6 & 0.073 & 2.06 & 0.58 & 0.451 & 0.66 & 5.15 & 0.081 & 0.046 & 123 \\
\hline DP17960 & 15 & 1 & 1.26 & 129.26 & 0.60 & 1.84 & 7.51 & 25.6 & 0.078 & 2.23 & 0.48 & 0.423 & 0.83 & 5.00 & 0.095 & .036 & 104 \\
\hline DP17961 & 15 & 1 & 1.46 & 129.46 & 0.65 & 1.86 & 7.47 & 25.2 & 0.072 & 2.37 & 0.42 & 0.402 & 0.81 & 5.01 & 0 . & 43 & 93 \\
\hline DP17962 & 15 & 2 & 0.06 & 129.56 & 0.59 & 1.87 & 7.42 & 25.6 & 0.068 & 2.22 & 0.46 & 95 & 0.65 & 4.82 & 0.0 & 0.043 & 105 \\
\hline 7963 & 15 & 2 & 0.26 & 129.76 & 0.64 & 1.86 & 7.45 & 25.4 & 0.069 & 2. & 0.49 & 0.408 & 0.80 & 5.09 & 0. & 0. & 96 \\
\hline DP17964 & 15 & 2 & 0.46 & 129.96 & 0.67 & 1.80 & 7.42 & 25.7 & 0.062 & 2.37 & 0.45 & 0.402 & 0.73 & 4.85 & 0.088 & & 97 \\
\hline DP17965 & 15 & 2 & 0.66 & 130.16 & 0.65 & 1.80 & 7.50 & 25.6 & 0.066 & 2.38 & 0.45 & 0.401 & 0.75 & 4.89 & 0.092 & 0.040 & 93 \\
\hline DP17966 & 15 & 2 & 0.86 & 130.36 & 0.59 & 1.81 & 7.42 & 25.5 & 0.066 & 2.17 & 0.50 & 0.394 & 0.71 & 5.08 & 0 . & 0.033 & 106 \\
\hline DP17967 & 15 & 2 & 1.06 & 130.56 & 0.61 & 1.76 & 7.49 & 25.7 & 0.066 & 2.41 & 0.39 & 0.403 & 0 & 4 & 0 . & 0.033 & 88 \\
\hline & 15 & 2 & 1.26 & 130.76 & 0.63 & 1.79 & 7.44 & 25.5 & 0.071 & 2.36 & 0.44 & 0.402 & 0.68 & 4.91 & 0.087 & 0.040 & 91 \\
\hline DP17969 & 15 & 2 & 1.46 & 130.96 & 0.60 & 1.84 & 7.45 & 25.7 & 0.069 & 2.29 & 0.42 & 0.402 & 0.65 & 5.00 & 0.087 & 0.028 & 102 \\
\hline DP17970 & 15 & 3 & 0.06 & 131.06 & 0.61 & 1.85 & 7.42 & 25.6 & 0.070 & 2.40 & 0.40 & 0.413 & 0.62 & 4.98 & 0.089 & 0.031 & 92 \\
\hline DP17971 & 15 & 3 & 0.26 & 131.26 & 0.60 & 1.83 & 7.44 & 25.6 & 0.065 & 2.37 & 0.38 & 0.403 & 0.65 & 4.94 & 0.084 & 0.032 & 92 \\
\hline DP17972 & 15 & 3 & 0.46 & 131.46 & 0.64 & 1.84 & 7.49 & 25.7 & 0.068 & 2.40 & 0.41 & 0.410 & 0.68 & 4.95 & 0.086 & 0.045 & 92 \\
\hline & 15 & 3 & 0.66 & 131.66 & 0.63 & 1.84 & 7.51 & 25.6 & 0.069 & 2.35 & 0.40 & 0.407 & 0.81 & 5.05 & 0.089 & 0.046 & 97 \\
\hline 7974 & 15 & 3 & 0.86 & 131.86 & 0.63 & 1.83 & 7.53 & 25.5 & 0.070 & 2.32 & 0.40 & 0.404 & 0.82 & 4.93 & 0.091 & 0.047 & 99 \\
\hline DP17975 & 15 & 3 & 1.06 & 132.06 & 0.62 & 1.84 & 7.53 & 25.2 & 0.075 & 2.31 & 0.40 & 0.404 & 0.85 & 5.07 & 0.093 & 0.052 & 98 \\
\hline DP17976 & 15 & 3 & 1.26 & 132.26 & 0.62 & 1.84 & 7.50 & 25.4 & 0.067 & 2.32 & 0.40 & 0.416 & 0.81 & 5.15 & & & 98 \\
\hline DP17977 & 15 & 3 & 1.46 & 132.46 & 0.53 & 1.93 & 7.52 & 25.7 & 0.065 & 2.30 & 0.32 & 0.389 & 0.72 & 4.84 & 0.081 & 0.037 & 101 \\
\hline DP17 & 15 & 4 & 0.06 & 132.56 & 0.53 & & 7.41 & 26.1 & 0.050 & 1.57 & 0.33 & 0.278 & 0.46 & 3.79 & 0.060 & 0.044 & 138 \\
\hline & 15 & 4 & 0.26 & 132.76 & 0.51 & & 7.35 & 25.2 & 0.053 & 2.04 & 0.40 & 0.412 & 0.73 & 5.62 & 0.076 & 0.029 & 122 \\
\hline DP17980 & 15 & 4 & 0.46 & 132.96 & 0.55 & 1.84 & 7.51 & 25.3 & 0.063 & 2.31 & 0.35 & 0.406 & 0.72 & 5.21 & 0.085 & 0.045 & 104 \\
\hline
\end{tabular}


Table 6. (Continued).

\begin{tabular}{|c|c|c|c|c|c|c|c|c|c|c|c|c|c|c|c|c|c|}
\hline Lab ID & பّ & 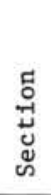 & 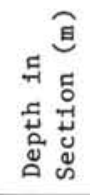 & 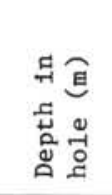 & $\mathrm{Na}$ & $\mathrm{Mg}$ & A1 & $\mathrm{S} 1$ & $\mathrm{P}$ & K & $\mathrm{Ca}$ & Ti & Mn & $\mathrm{Fe}$ & $\mathrm{Ba}$ & $\mathrm{S}$ & $\mathrm{H}_{2} \mathrm{O}$ \\
\hline 7981 & 15 & 4 & .66 & 16 & 0.56 & 1.77 & 7.61 & 3 & 0.068 & 2.41 & 0.33 & 0.411 & 0.85 & 5.11 & 0.091 & .035 & 92 \\
\hline 17982 & 15 & 4 & 0.86 & 133.36 & 0.56 & 1.79 & 7.62 & 25.4 & 0.067 & 2.51 & .31 & 0.417 & 0.76 & & & & 8 \\
\hline DP17983 & 15 & 4 & 1.06 & 133.56 & 0.55 & 1.84 & 7.57 & 25.4 & 0.060 & 2.45 & 0.32 & 0.423 & 0.65 & 5.29 & 0.080 & 0 & 92 \\
\hline DP17984 & 15 & 4 & 1.26 & 133.76 & 0.55 & 1.90 & 7.50 & 25.3 & 0.058 & 2.21 & 0.43 & 0.433 & 0.68 & 5.65 & 0.077 & 0.046 & 111 \\
\hline P17985 & 15 & 4 & 1.46 & & 0.54 & 1.97 & 7.37 & 25.3 & 0.054 & 1.96 & 0.45 & 0.428 & 0.65 & 5.96 & 0.074 & 0.042 & 127 \\
\hline DP17986 & 15 & 5 & 0.06 & 13 & 0.57 & 1.85 & 7.63 & 25.4 & 0.066 & 2.57 & 0.33 & 0.429 & 0.63 & 5.23 & 0.081 & 0.050 & 86 \\
\hline DP17987 & 15 & 5 & 0.26 & 134.26 & 0.56 & 1.85 & 7.67 & 25.6 & 0.072 & 2.49 & 0.33 & 0.416 & 0.74 & 5.04 & 0.084 & 0.047 & 91 \\
\hline DP17988 & 15 & 5 & 0.46 & 134.46 & 0.53 & 1.96 & 7.54 & 25.4 & 0.066 & 2.18 & 0.33 & 0.419 & 0.65 & 5.20 & 0.077 & .051 & 113 \\
\hline DP17989 & 15 & 5 & 0.66 & 134.66 & 0.50 & 1.95 & 7.52 & 25.2 & 0.061 & 2.02 & 0.39 & 0.422 & 0.65 & 5.73 & 0.075 & .044 & 123 \\
\hline DP17990 & 15 & 5 & 0.84 & $13 t$ & 0.52 & 1.91 & 7.66 & 25.5 & 70 & & 0 & 03 & 0.75 & & & & 107 \\
\hline DP17991 & 15 & 5 & 1.00 & 135.00 & 0.58 & 1.87 & 7.78 & 25.4 & 0.070 & 2.15 & 0.36 & 0.415 & 0.72 & 5.08 & & 42 & 109 \\
\hline DP17992 & 15 & 5 & 1.16 & 135.16 & 0.58 & 1.84 & 7.80 & 25.3 & 0.067 & 2.17 & 0.40 & 0.418 & 0.73 & 5.31 & 0. & 0.035 & 109 \\
\hline DP17993 & 15 & 6 & 0.06 & 135.56 & 0.60 & 1.81 & 7.83 & 25.3 & 0.074 & 2.34 & 0.32 & 0.408 & 0.83 & 5.04 & 0.090 & 0.047 & 95 \\
\hline DP17994 & 15 & 6 & 0.26 & 135.76 & 0.55 & 1.93 & 7.75 & 25.5 & 0.065 & 1.99 & 0.34 & 0.406 & 0.69 & 5.04 & & & 115 \\
\hline 995 & 15 & 6 & 0.46 & 135.96 & 0.62 & 1.83 & 7.86 & 25.5 & 0.072 & 2.34 & 0.30 & 0.391 & 0.86 & 4.88 & 0.087 & 0.051 & 92 \\
\hline DP17996 & 15 & 6 & 0.66 & 136.16 & 0.60 & 1.85 & 7.84 & 25.4 & 0.064 & & 0.28 & 0.396 & 0.72 & & 0.083 & & 90 \\
\hline 7997 & 15 & 6 & 0.86 & 13 & 0.64 & 1.84 & 7.84 & 25.5 & 0.066 & 2.39 & 0.30 & 0.403 & 0.83 & 4.89 & 8 & & 89 \\
\hline 998 & 15 & 6 & 1.06 & 13 & 0.64 & 1.82 & 7.8 & 25.4 & 0.064 & 2.47 & 0.28 & 0.412 & 0.71 & 5.00 & 0. & & 85 \\
\hline DP17999 & 15 & 6 & 1.16 & & 0.61 & 1.82 & 7.82 & $25.4^{\circ}$ & 0.064 & 2.33 & 0.30 & 0.411 & 0.68 & 5.16 & 0. & 5 & 95 \\
\hline DP1 8000 & 15 & 6 & 1.46 & & 0.63 & 1.84 & 7.77 & 25.3 & 0.065 & 2.30 & 0.31 & 0.409 & 0.68 & 5.17 & 0. & & 95 \\
\hline DP18002 & 15 & 7 & 0.26 & 137.26 & 0.59 & 1.82 & 7.82 & 25.2 & 0.073 & 2.35 & 0.32 & 0.416 & 0.77 & & 89 & & 94 \\
\hline 03 & 15 & 7 & 0.45 & 13 & 0.60 & 1.80 & 7. & 25.2 & 0. & 2.28 & 0.31 & 0.416 & 0.81 & 5.18 & 36 & 8 & 100 \\
\hline 004 & 16 & 1 & 0.36 & & 0.57 & 1.88 & 7.86 & 25.3 & 0.071 & 2.25 & 0.33 & 0.424 & 0.75 & 5.27 & & 0. & 112 \\
\hline DP18005 & 16 & 1 & 0.56 & 13 & 0.56 & 1.91 & 7.93 & 25.1 & 0.073 & 2.25 & 2 & 0.420 & 0.74 & 5. & 6 & & 108 \\
\hline DP18006 & 16 & 1 & 0.76 & & 0.50 & 1.89 & 7.75 & 25.2 & 0.075 & 2.19 & 0 & 0.410 & 0.76 & 5.03 & & & 120 \\
\hline DP18007 & 16 & 1 & 0.96 & & 0.58 & 1.86 & 7.80 & 25.4 & 0.072 & & 0 & 8 & 0.70 & & & & 111 \\
\hline 08 & 16 & 1 & 1.16 & 13 & 0.60 & 1.81 & 7. & 25 & 0 & 2 & 1 & 8 & 0.72 & 5.11 & 0. & 0 . & 105 \\
\hline 009 & 16 & 1 & 1.36 & 13 & 0.65 & 1.81 & 7. & 25.3 & 0.077 & 2.39 & 0.31 & 0.411 & 0.77 & 5.03 & 0.095 & 0.060 & 91 \\
\hline 3010 & 16 & 2 & 0.06 & & 0.61 & 1.82 & 7.91 & 25.2 & 0.069 & 2.36 & 0.30 & 0.420 & 0.65 & 5.18 & 0. & 0. & 93 \\
\hline DP18011 & 16 & 2 & 0.26 & 51 & 0.60 & 1.79 & 7.93 & 25.3 & 0.072 & 2.50 & 0.30 & 0.422 & 0.60 & 5.17 & 33 & 7 & 4 \\
\hline DP18012 & 16 & 2 & 0.46 & 139.71 & 0.61 & 1.80 & 8.00 & 25.4 & 0.071 & 2.51 & 0.30 & 0.427 & 0.61 & 5.36 & 85 & 48 & 32 \\
\hline DP18013 & 16 & 2 & 0.66 & & 0.61 & 1.79 & 7.89 & 25.2 & 0.074 & & 0.30 & 0.421 & 0.73 & & & & 8 \\
\hline & 16 & 2 & 0.86 & & 0 & & 8. & & 0 & & 1 & 30 & 58 & 5 & 37 & 52 & 82 \\
\hline 15 & 16 & 2 & 1.06 & 14 & 0. & 1.76 & 7. & 25 & 0.078 & 2. & 0.31 & 0.414 & 0.66 & 5.19 & 0.084 & 0.053 & 86 \\
\hline 16 & 16 & 2 & 1.26 & 140.51 & 0.62 & 1.79 & 7.82 & 24.9 & 0.071 & 2.31 & 0.31 & 0.422 & 0.67 & 5.58 & 0. & 0. & 94 \\
\hline DP18017 & 16 & 2 & 1.46 & 140.71 & 0.63 & 1.78 & 7.92 & 25.1 & 0.077 & 2.37 & .31 & 0.414 & 0.73 & 5.21 & 0.089 & 58 & 87 \\
\hline DP18018 & 16 & 3 & 0.06 & 140.81 & 0.62 & 1.78 & 7.95 & 25.2 & 0.073 & 2.32 & 0.31 & 0.408 & 0.71 & 5.20 & & & 90 \\
\hline 8019 & 16 & 3 & 0.26 & 141.01 & 0.65 & 1.78 & & 25.2 & & & & 0.419 & 0.59 & & & & 9 \\
\hline D: & 16 & 3 & 0.46 & .21 & 5 & 1 & 8. & 25 & 5 & 2 & 3 & 8 & 0.76 & 5.12 & 0.089 & 0. & 88 \\
\hline 021 & 16 & 3 & 0.66 & 141.41 & 0.65 & 1.74 & 8. & 24.9 & 0.085 & 2.43 & 0.35 & 0.422 & 0.78 & 5.36 & 0.097 & 0.058 & 81 \\
\hline DP18022 & 16 & 3 & 0.86 & 141.61 & 0.64 & 1.74 & 8. & 24.9 & 0.082 & 2.27 & .36 & 0.426 & 0.81 & 5.54 & 0.092 & & 89 \\
\hline DP18023 & 16 & 3 & 1.06 & 141.81 & 0.64 & 1.69 & 8.20 & 25.0 & 0.095 & 2.43 & 0.37 & 0.417 & 0.83 & 5.17 & & & 7 \\
\hline DP18024 & 16 & 3 & 1.26 & 142.01 & 0.66 & 1.70 & 8.19 & 25.0 & 0.087 & 2.46 & 0.35 & 0.423 & 0.77 & 5.25 & & & 79 \\
\hline DP18025 & 16 & 3 & 1.46 & 142.21 & & 1.78 & & 24.9 & & & & & & & & 0 & 35 \\
\hline DP & 16 & 4 & 0.06 & 31 & & 1 & 8. & 2 & 8 & 2 & 3 & 30 & 0.67 & 5.34 & 0.088 & 0. & 80 \\
\hline & 16 & 4 & 0.26 & .51 & 0 & 1.78 & 8. & 25.2 & 0.080 & 2.47 & 0.32 & 0.425 & 0.67 & 5.21 & 0.086 & 0.057 & 83 \\
\hline DP18028 & 16 & 4 & 0.46 & 142.71 & 0.57 & 1.81 & 8.10 & 25.0 & 0.077 & 2.29 & 0.31 & 0.404 & 0.65 & 4.98 & 0.083 & 0.049 & 97 \\
\hline DP18029 & 16 & 4 & 0.66 & 142.91 & 0.60 & 1.80 & 8.18 & 25.1 & 0.080 & 2.43 & 0.31 & 0.409 & 0.72 & 5.06 & 0.087 & & 6 \\
\hline DP1 8030 & 16 & 4 & 0.86 & 143.11 & 0.55 & 1.90 & 8.15 & 25.3 & 0.076 & 2.13 & 0.30 & 0.388 & 0.61 & 4.72 & 0.0 & & 107 \\
\hline DP18031 & 16 & 4 & 1.06 & 143.31 & 0.63 & 1. & 8.10 & 25.0 & & & & & & & & & 30 \\
\hline DP18032 & 16 & 4 & 1.26 & 143.51 & & 1.93 & 7.97 & 25.1 & & & & 33 & 0.66 & 5. & 0. & 0. & 106 \\
\hline & 16 & 4 & 1.46 & & & 2.00 & 7. & 25.1 & 0 & 2. & 0.30 & 0.387 & 0.71 & 4.80 & 0. & 0. & 114 \\
\hline & 16 & 5 & 0.06 & & 0. & & & 25.4 & & 1. & 0 & 0.339 & 0.63 & 3.81 & 0. & & 141 \\
\hline DP18035 & 16 & 5 & 0.26 & 144.01 & 0.62 & 1.93 & 7.82 & 24.9 & 0.092 & 2.09 & & & 0.85 & & & & 108 \\
\hline DP18036 & 16 & 5 & 0.46 & 144.21 & 0.64 & 1.88 & 7.94 & 24.6 & 0.081 & 2.27 & & & 1.07 & & & & \\
\hline DP18037 & 16 & 5 & 0.66 & & & & & 24.5 & & & & & & & 0 & & 0 \\
\hline & 16 & 5 & 1.06 & 144.81 & 0.62 & & 7.80 & 25.1 & 0.095 & 2.16 & 0.40 & 0.418 & 0.86 & 5.00 & 0.077 & 0 . & 99 \\
\hline & 16 & 5 & 1.26 & & 0.63 & 1.96 & 7.78 & 25.0 & 0.101 & 2. & 0.42 & 0.414 & 1.08 & 5.11 & 0.083 & 0.067 & 106 \\
\hline & 16 & 5 & 1.46 & 145.21 & 0.6 & & 7. & 25.0 & 0.105 & 2.13 & 0.41 & 0.412 & 0.91 & 5.30 & 0.078 & 0.060 & 95 \\
\hline DP18042 & 16 & 6 & 0.07 & $145 \cdot 32$ & 0.65 & 1.83 & 7.89 & 25.3 & 0.110 & 2.23 & 0.41 & 0.394 & 0.94 & & & & 1 \\
\hline DP18043 & 16 & 6 & 0.21 & 145.46 & 0.64 & 1.91 & 7.91 & 25.3 & 0.079 & 2.19 & 0.33 & 0.393 & 0.80 & 4.87 & & & 95 \\
\hline 8044 & 17 & 1 & & & & & & 24.0 & & & & & 1.23 & & & & 14 \\
\hline & 17 & 1 & 0.48 & & & & & 24.1 & & & 0.49 & 0.434 & 1.23 & 5.31 & 0.0 & & 89 \\
\hline DP18046 & 17 & 1 & .67 & 147.69 & 0.69 & 1.78 & 8.13 & 24.0 & 0.137 & 2.5 & 0.53 & 0.443 & 1.24 & 5.45 & 0.071 & 0.068 & 8 \\
\hline
\end{tabular}




\begin{tabular}{|c|c|c|c|c|c|c|c|c|c|c|c|c|c|c|c|c|c|}
\hline Lab ID & ర్ & & 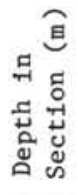 & 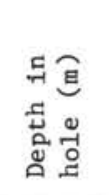 & $\mathrm{Na}$ & $\mathrm{Mg}$ & A1 & Si & P & K & $\mathrm{Ca}$ & T1 & $\mathrm{Mn}$ & $\mathrm{Fe}$ & $\mathrm{Ba}$ & S & $\mathrm{H}_{2} \mathrm{O}$ \\
\hline 047 & 17 & 1 & 86 & 88 & 5 & 6 & 12 & 24.2 & 35 & 61 & 0.52 & 31 & 1.38 & 5.32 & 72 & 60 & 86 \\
\hline 18048 & 7 & 1 & 06 & & 61 & & & & & & & & & & & & 91 \\
\hline 049 & 17 & 1 & .26 & & .57 & 1 & & & & & 0.49 & 6 & 1.51 & & .070 & & \\
\hline & 17 & 1 & 1.46 & & & 1.76 & .06 & 23.9 & & .53 & 0.47 & & 1.52 & & .072 & & \\
\hline & 17 & 2 & 0.11 & & & 1.77 & & & & & & & & & .073 & & \\
\hline 2 & 17 & 2 & 0.43 & & & & & & & & & & & & & & \\
\hline 053 & 17 & 2 & 0.55 & 49.07 & .61 & & & & & & & & & & & & \\
\hline 18054 & 17 & 2 & 0.71 & 49.23 & .61 & 1.76 & .07 & 23.9 & .126 & & 0.47 & & 1.62 & & .075 & & 88 \\
\hline & 17 & 2 & 0.86 & 49.38 & 0.60 & 1.79 & .03 & 4.0 & 123 & 55 & 0.48 & & 1.63 & 0 & & & \\
\hline & 17 & 2 & 1.06 & & & 1.77 & .02 & 24.0 & & 61 & 0.42 & & 5 & & & & \\
\hline & 17 & 2 & 1.16 & & & & & & & & & & & & & & \\
\hline & 17 & 2 & 1 & & & & & & & & & & & & & & \\
\hline & 17 & 3 & .00 & 50.08 & 9 & & & & & & & & & & & & \\
\hline 60 & 17 & 3 & .26 & 50.28 & .63 & & & & & & 40 & & 5 & & & & 7 \\
\hline & 17 & 3 & .46 & 50.48 & .03 & 1.7 & .96 & 23.9 & 1 & 8 & 0.47 & .431 & 10 & 36 & & & \\
\hline & 17 & 3 & 0.66 & 50.68 & .63 & 1.78 & .99 & 24.1 & 100 & 69 & 0.40 & & & & & & \\
\hline & 17 & 3 & 0.82 & 50.84 & & 1.73 & & 23. & & & & & & & & & \\
\hline & 17 & 3 & 0.99 & & & & & & & & & & & & & & \\
\hline & 17 & 3 & & & & & & & & & & & & & & & \\
\hline & 17 & 3 & 1.34 & .36 & .54 & 1.7 & & 2 & & & & & & & & & \\
\hline & 17 & 4 & 0.06 & 51.58 & .60 & 1.69 & .97 & 23.5 & (29 & 3 & .58 & 22 & 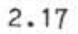 & 1 & & & 103 \\
\hline & 17 & 4 & 0.26 & & 0.67 & & & 23. & & & & & & & & & \\
\hline & 17 & 4 & 0.46 & 51.98 & 8 & & & & & & & & & & & & \\
\hline & 17 & 4 & 0 & & & & & & & & & & & & & & \\
\hline & 1 & 4 & .82 & & . & 1.8 & 2 & .0 & & & & & & & & & 9 \\
\hline & If & 4 & 1.00 & 52.52 & 0.59 & 1.8 & 7.41 & 22.6 & 276 & & .89 & 45 & 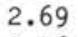 & 3 & 5 & & \\
\hline & 17 & 4 & 1.18 & & & & & & & & & & & & & & \\
\hline & 17 & 4 & & & & & & & & & & & & & & & 15 \\
\hline & 17 & 5 & 0 & & & & & & & & & & & & & & 9 \\
\hline 76 & 17 & 5 & 0.26 & & 1 & 1. & & 2 & & & & & & & & & 103 \\
\hline & 17 & 5 & 0.46 & 48 & 0 & 1.8 & 1 & 22. & & & - & & & & & & 103 \\
\hline & 17 & 5 & 0.66 & & & & & & & & & & & & & & \\
\hline & 17 & 5 & & & & & & & & & & & & & & & \\
\hline & 17 & 5 & & & & & & & & & & & & & & & 97 \\
\hline & 17 & 5 & 1.26 & & 1 & & & 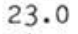 & & & & & & & & & \\
\hline & 18 & 1 & 0.26 & .86 & .58 & 1. & 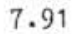 & 24.0 & & & 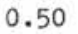 & & 1. & 7 & & & \\
\hline & 18 & 1 & 0.46 & 06 & 0.5 & 1.74 & 2 & 24. & & & 0. & & & & & & \\
\hline & 18 & 1 & 0.66 & & 6 & 1 & & 23.9 & & & & & & & & & \\
\hline & 18 & 1 & & & & & & & & & & & & & & & \\
\hline & 18 & 1 & & & & & & & & & & & & & & & \\
\hline 7 & 18 & 1 & 1.26 & & 3 & 1.80 & 4 & 4.0 & & & 0 & & & & & & \\
\hline & 18 & 1 & 1.46 & & 0.51 & 1.8 & 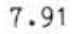 & 23.9 & & & 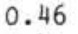 & & & & & & \\
\hline & 18 & 2 & 0.06 & & 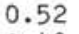 & & & 3. & & & & & & & & & \\
\hline & 18 & 2 & & & & & & & & & & & & & & & \\
\hline & 18 & 2 & & & & & & & & & & & & & & & \\
\hline & 18 & 2 & 36 & & 4 & & & $=$ & & & & & & & & & \\
\hline & 18 & 2 & 1.06 & & 7 & & & . & & & 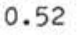 & & & & & & \\
\hline & 18 & 2 & 1.26 & .36 & 0.3 & 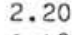 & 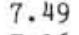 & 4. & & & c & & & & & & \\
\hline & 18 & 2 & & & & & & & & & & & & & & & \\
\hline & 18 & 3 & & & & & & & & & & & & & & & \\
\hline & 18 & 3 & & & & & & & & & & & & & & & \\
\hline & 18 & 3 & & & 0 & & & 24.6 & & & & & & & & & \\
\hline & 18 & 3 & 0.66 & & & & & 4.5 & & & & & & & & & \\
\hline P18101 & 18 & 3 & 0.86 & .46 & 0.41 & 2.1 & & 24.6 & & & & & & 9 & & & \\
\hline & 18 & 3 & 0 & & & & & 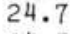 & & & & & & & & & \\
\hline & 18 & 3 & & & & & & & & & & & & & & & \\
\hline & 18 & 4 & 0.06 & & & & & 24 & & & & & & & & & \\
\hline & 18 & 4 & & & & & & 848 & & & & & & & & & \\
\hline 06 & 18 & 4 & 0.46 & & 4 & 8 & & 4. & & & & & & & & & \\
\hline 18107 & 18 & 4 & 0.66 & 61.76 & .40 & 2.2 & & 24.7 & 1 & 3 & & & & & 71 & & \\
\hline & 18 & 4 & & & 0.40 & 2.2 & & 24. & & & & & & & & & \\
\hline & 18 & 4 & 1.06 & & 0.42 & 2.15 & & 24.2 & & & & & & & & & \\
\hline & 18 & 4 & 1.26 & & 0. & & & & & & & & & & & & \\
\hline & 1 & C & & & & & & & & & & & & & 6 & 0 & \\
\hline
\end{tabular}


G. R. HEATH, R. B. KOVAR, C. LOPEZ, G. L. CAMPI

Table 6. (Continued).

\begin{tabular}{|c|c|c|c|c|c|c|c|c|c|c|c|c|c|c|c|c|c|}
\hline Lab ID & Нّ & 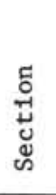 & 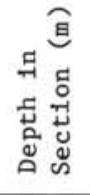 & 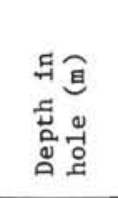 & $\mathrm{Na}$ & $\mathrm{Mg}$ & Al & Si & P & K & $\mathrm{Ca}$ & $\mathrm{Ti}$ & Mn & $\mathrm{Fe}$ & $\mathrm{Ba}$ & $\mathrm{s}$ & $\mathrm{H}_{2} \mathrm{O}$ \\
\hline DP18112 & 18 & $C$ & 0.30 & 162.73 & 0.38 & 2.18 & 7.02 & 23.8 & 0.342 & 3.00 & 0.97 & 0.419 & 1.26 & 4.96 & 0.082 & 0.064 & 66 \\
\hline DP18113 & 19 & 1 & 0.47 & 166.34 & 0.38 & 2.29 & 7.51 & .7 & 0.162 & 3.14 & 0.51 & 0.405 & 1.04 & 5.05 & 0.071 & 0.053 & 69 \\
\hline DP18114 & 19 & 1 & 0.66 & 166.53 & 0.39 & 2.21 & 7.31 & 24.4 & 0.244 & 3.14 & 0.72 & 0.407 & 1.11 & 4.99 & 0.072 & 0.055 & 67 \\
\hline DP18115 & 19 & 1 & 0.86 & 166.73 & 0.41 & 2.18 & 7.35 & 24.5 & 0.271 & 3.12 & 0.79 & 0.412 & 1.10 & 4.99 & 0.070 & 0.061 & 67 \\
\hline DP18116 & 19 & 1 & 1.06 & 166.93 & 0.38 & 2.16 & 7.16 & 24.1 & 0.276 & 3.06 & 0.79 & 0.395 & 1.14 & 4.91 & 0.072 & 0.057 & 66 \\
\hline 3117 & 19 & 1 & 1.26 & 167 & 0.37 & 2.21 & 7.09 & 24.2 & 0.292 & 3.04 & 0.83 & 0.425 & 1.10 & 4.93 & 0.072 & 0.059 & 67 \\
\hline DP18118 & 19 & 1 & 1.46 & 167 & 0.37 & 2.17 & 7.03 & 23.8 & 0.346 & 2.99 & 0.98 & 0.418 & 1.19 & 4.93 & 0.072 & 0.065 & 68 \\
\hline DP18131 & 19 & 2 & 0.32 & 167.69 & 0.50 & 1.94 & 6.78 & 24.0 & 0.642 & 3.02 & 1.74 & 0.413 & 1.23 & 5.13 & 0.081 & 0.083 & 82 \\
\hline DP1 8132 & 19 & 2 & 0.48 & 167 & 0.53 & 1.97 & 7.00 & 23.2 & 0.735 & 2.90 & 2.05 & 0.435 & 1.50 & 5.17 & 0.074 & 0.102 & 98 \\
\hline DP18133 & 19 & 2 & 0.66 & 168.03 & 0.50 & 1.92 & 7.06 & 23.4 & 0.695 & 2.96 & 1.89 & 0.419 & 1.29 & 5.21 & 0.075 & 0.087 & 68 \\
\hline DP18134 & 19 & 2 & 0.86 & 168 & 0.50 & 1.97 & 7.04 & 23.5 & 0.677 & 3.02 & 1.78 & 0.414 & 1.38 & 5.15 & 0.075 & .085 & 68 \\
\hline DP18119 & 19 & 2 & 0.99 & & 0.52 & 1.94 & 7.03 & 23.6 & 0.660 & 3.06 & 1.75 & 0.414 & 1.37 & 6 & 0.075 & 0.086 & 66 \\
\hline DP1 8120 & 19 & 2 & 1.11 & 168 & 0.49 & 1.94 & 7.08 & 23.6 & 0.654 & 3.02 & 1.72 & 0.404 & 1.32 & 5.10 & 0.073 & 0.081 & 65 \\
\hline DP18121 & 19 & 2 & 1.25 & 168.62 & 0.48 & 1.94 & 7.14 & 23.8 & 0.568 & 3.09 & 1.53 & 0.406 & 1.23 & 5.02 & 0.075 & 0.080 & 64 \\
\hline DP18122 & 19 & 2 & 1.38 & 16 & 0.4 & 1.94 & 7.07 & 23.7 & 0.557 & 3.08 & 1.55 & 0.401 & 1.19 & 8 & 0.075 & 0. & 65 \\
\hline DP18123 & 19 & 3 & 0.06 & 168 & 0.48 & 1.94 & 7.11 & 23.9 & 0.522 & 3.11 & 1.47 & 0.412 & 1.24 & 5.14 & 0.076 & 0.074 & 64 \\
\hline DP18124 & 19 & 3 & 0.26 & & 0.49 & 1.95 & 7.03 & 23.9 & 0.490 & 3.12 & 1.45 & 0.412 & 1.30 & 5 & 0.078 & 0.072 & 65 \\
\hline DP18125 & 19 & 3 & 0.46 & & 0.46 & 1.95 & 7.03 & 24.0 & 0.490 & 3.12 & 1.44 & 0.411 & 1.30 & 5.21 & 0.078 & 0.068 & 65 \\
\hline DP18126 & 19 & 3 & 0.66 & 169 & 0.51 & 1.93 & 6.97 & 24.0 & 0.601 & 3.10 & 1.68 & 0.419 & 1.25 & 5.15 & 0.078 & 0.081 & 64 \\
\hline DP18127 & 19 & 3 & 0.86 & & 0.55 & 1.85 & 6.92 & 23.7 & 0.637 & 3.06 & 1.77 & 0.410 & 1.38 & 5.14 & 0.078 & 0.085 & 66 \\
\hline DP1 8128 & 19 & 3 & 1.06 & 169 & 0.52 & 1.89 & 6.84 & 23.8 & 0.699 & 3.06 & 1.89 & 0.410 & 1.30 & 5.11 & 0.077 & 0.089 & 64 \\
\hline DP18129 & 19 & C & 0.05 & 170.12 & 0.53 & 1.92 & 6.80 & 23.9 & 0.667 & 3.03 & 1.84 & 0.410 & 1.25 & 5.11 & 0.077 & 0.088 & 65 \\
\hline DP18130 & 19 & $\mathrm{C}$ & 0.29 & 170.36 & 0.37 & 2.10 & 7.05 & 23.9 & 0.470 & 2.94 & 1.32 & 0.428 & 1.32 & 5.05 & 0.074 & 0.072 & 64 \\
\hline DP17952 & 20 & 1 & 0.06 & & 0.36 & 2.17 & 6.15 & 23.7 & 0.574 & 3.08 & 1.68 & 0.354 & 1.77 & 5.38 & 0.113 & 0.076 & 101 \\
\hline DP17953 & 20 & 1 & 0.22 & 176.02 & 0.58 & 2.08 & 5.97 & 23.4 & 0.995 & 3.07 & 2.60 & 0.353 & 1.39 & 4.70 & 0.112 & 0.097 & 73 \\
\hline DP17954 & 20 & 1 & 0.46 & 176.26 & 0.71 & 1.90 & 5.94 & 24.1 & 0.803 & 3.21 & 2.14 & 0.337 & 1.02 & 4.10 & 0.084 & 0.086 & 63 \\
\hline DP17955 & 20 & 1 & 0.71 & 176.51 & 0.67 & 2.07 & 5.74 & 23.1 & 1.282 & 2.88 & 3.24 & 0.346 & 1.32 & 4.40 & 0.091 & 0.110 & 61 \\
\hline
\end{tabular}

